\title{
Evaluation of precoding and feedback quantization schemes for multiuser communications systems
}

FERNANDO DOMENE OLTRA 


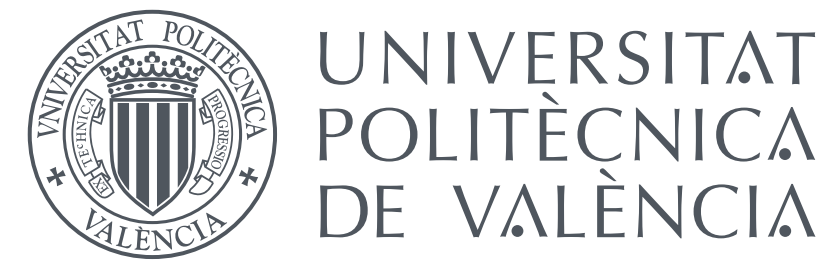

Evaluation of precoding and feedback quantization schemes for multiuser communications systems

\section{DOCTORAL THESIS \\ by}

Fernando Domene Oltra

Supervisor:

Dr. Gema Piñero Sipán

Valencia, Spain

December 2014 
Collection doctoral thesis

(c) Fernando Domene Oltra

(c) 2015, of the present edition: Editorial Universitat Politècnica de València Telf.: 963877012 / www.lalibreria.upv.es

ISBN: 978-84-9048-329-9 (printed version)

Any unauthorized copying, distribution, marketing, editing, and in general any other exploitation, for whatever reason, of this piece of work or any part thereof, is strictly prohibited without the authors' expressed and written permission.

Print in Spain 


\section{Abstract}

Multiple-input multiple-output (MIMO) communication systems have emerged as one of the most promising technologies in the field of wireless communications, allowing to exploit the spatial dimension as well as the time and frequency dimensions. Thus, higher rates can be obtained by using the same bandwidth, which is a scarce resource, and keeping a low transmit power, which is crucial in batteryoperated devices. For these reasons, MIMO technologies have been adopted by many standards such as Long-Term Evolution (LTE), LTE advanced (LTE-A) and Worldwide Interoperability for Microwave Access (WiMAX).

MIMO techniques can also be used in a multiuser scenario, where several users share the spatial dimension causing multiuser interference. By means of precoding and the use of multiple antennas at the transmitter, the signal of the different users can be spatially multiplexed so that multiuser interference is mitigated even for single-antenna users. These systems, known as multiuser multiple-input singularoutput (MU-MISO) systems, have attracted much attention in recent years since they allow the development of small and inexpensive terminals, keeping the most expensive hardware at the transmitter.

However, these benefits come at the cost of having a more complex system. On the one hand, spatial multiplexing requires a considerable processing load that depends on the size of the system: number of transmit antennas, number of receivers and bandwidth. On the other hand, MIMO techniques require accurate channel state information at the transmitter (CSIT). In frequency-division duplex (FDD) systems, channel state information (CSI) has to be estimated at the receiver and provided to the transmitter through the feedback link, hence reducing the efficiency of the system. Therefore, this thesis is primarily focused on improving the efficiency of precoding implementations and the performance of feedback schemes in MU-MISO systems.

First, the problem of precoding is addressed. An analysis of some of the most utilized precoding techniques is conducted, paying special attention to their performance and computational complexity. The analysis reveals that those techniques that make use of lattice reduction (LR) achieve the best performance. However, the 
computational complexity of LR makes its implementation difficult for practical systems. The analysis reveals that zero-forcing (ZF), Tomlinson-Harashima precoding (THP) and lattice reduction Tomlinson-Harashima precoding (LR-THP) are the most suitable techniques for covering the entire range of performance and computational complexity. An analysis of these techniques with imperfect CSIT has also been performed. In this analysis, LR has proven to be a key technique also when imperfect CSIT is available.

Next, parallel implementations of the precoding techniques on a graphic processing unit (GPU) are presented and compared to implementations that use a central processing unit (CPU). Since the implementations of THP and LR-THP have shown to best fit the GPU architecture and since they also share many operations, a GPU implementation of a reconfigurable THP scheme combined with LR has been proposed. The reconfigurable nature of GPUs allows gating the LR stage off when the user requirements are sufficiently guaranteed by the THP scheme, trading computational cost and performance. Although this implementation achieves a significant speed-up compared to its CPU implementation, its parallelism is limited by the sequential nature of LR. Therefore, several strategies for the parallelization of the LR problem are proposed and evaluated on different platforms: multicore CPU, GPU and a heterogeneous platform consisting of CPU+GPU. Results reveal that a GPU architecture allows a considerable reduction of the computational time of the LR problem, especially in the heterogeneous platform.

The second part of this thesis addresses the problem of feedback in FDD systems. In these systems, a quantized version of the channel is usually provided by the receivers through the feedback link. In order to keep a high efficiency, the channel must be quantized using as few bits as possible. First, the use of the frequency correlation to reduce the feedback information is explored. Two different schemes based on vector quantization (VQ) and the Karhunen-Loève (KL) transform, respectively, are presented and compared with existing schemes in terms of performance and complexity. Results show that both techniques are able to significantly reduce the feedback overhead by taking advantage of the frequency correlation.

Finally, the spatial correlation is leveraged to reduce the feedback information. A spatial statistical characterization of the spatial channel model (SCM) from the 3rd Generation Partnership Project (3GPP) for a highly correlated environment is presented. Based on this characterization, a channel quantization scheme for highly correlated environments is proposed. In order to obtain a statistical characterization for both high and low correlations, a simpler model such as the Kronecker correlation model is considered. Based on this characterization, two quantization schemes have been presented and evaluated using a realistic channel model such as the SCM. Results show that both schemes are able to reduce the feedback overhead in highly and moderately correlated scenarios. 
Keywords: wireless communications, mobile communications, MIMO, precoding, lattice reduction, GPU, limited feedback, channel quantization. 



\section{Resumen}

Los sistemas de comunicaciones con múltiples antenas o sistemas MIMO (multipleinput multiple-output) se presentan como una de las tecnologías más prometedoras en el campo de las comunicaciones inalámbricas, ya que permiten aprovechar la dimensión espacial además de las dimensiones de frecuencia y tiempo. De esta forma, se pueden obtener tasas de transmisión más elevadas usando el mismo ancho de banda, que es un recurso escaso, y manteniendo una potencia de transmisión baja, lo cual es crucial para dispositivos alimentados por baterías. Por estas razones, la tecnología MIMO ha sido adoptada en muchos estándares como Long-Term Evolution (LTE), LTE Advanced y Worldwide Interoperability for Microwave Access (WiMAX).

Las técnicas MIMO también pueden se pueden emplear en un escenario multiusuario, donde varios usuarios comparten la dimensión espacial provocando una interferencia multiusuario. A través de la precodificación y del uso de múltiples antenas en el transmisor, la señal de los diferentes usuarios puede ser multiplexada espacialmente de forma que se mitigue la interferencia multiusuario incluso con usuarios de una sola antena. Estos sistemas, conocidos como sistemas MUMISO (multiuser multiple-input single-output), han atraído mucho la atención en los últimos años ya que permiten el desarrollo de terminales pequeños y baratos, manteniendo así el equipamiento más caro en el transmisor.

Sin embargo, estos beneficios conllevan un sistema más complejo. Por una parte, el multiplexado espacial requiere una carga de procesado considerable, que depende del tamaño del sistema: número de antenas transmisoras, número de receptores y ancho de banda. Por otra parte, las técnicas MIMO requieren un conocimiento del canal en transmisión o CSIT (channel state information at the transmitter) preciso. En sistemas con duplexación por división en frecuencia o FDD (frequencydivision duplex), la información de canal o CSI (channel state information) debe ser estimada en el receptor y proporcionada al transmisor a través del enlace de realimentación, reduciendo así la eficiencia del sistema. Por lo tanto, esta tesis se centra en la mejora de la eficiencia de las implementaciones de precodificación y en el rendimiento de los esquemas de realimentación de canal en sistemas MU-MISO. 
El problema de la precodificación se aborda en primer lugar. Se ha llevado a cabo un análisis de algunas de las técnicas de precodificación más usadas, prestando especial atención a su rendimiento y a su complejidad. Este análisis revela que aquellas técnicas que hacen uso de lattice reduction (LR) obtienen un mejor rendimiento. Sin embargo, la complejidad computacional de la técnica LR dificulta su implementación en la práctica. El análisis también revela que las técnicas zeroforcing ( $\mathrm{ZF})$, Tomlinson-Harashima precoding (THP) y LR-THP son las técnicas más adecuadas para cubrir todo el rango de rendimiento y complejidad computacional. Asimismo, se ha llevado a cabo un análisis de estas técnicas bajo CSIT imperfecto. Dicho análisis ha demostrado que LR es una técnica muy importante también para el caso de CSIT imperfecto.

A continuación, se han presentado implementaciones paralelas de técnicas de precodificación sobre unidades de procesamiento gráfico o GPUs (graphic processing unit), comparándose con implementaciones en unidades de procesamiento central o CPU (central processing unit). Dado que las implementaciones de THP y LRTHP han demostrado ser las que mejor se adaptan a la arquitectura de la GPU y ya que tienen muchas operaciones comunes, se ha propuesto una implementación sobre GPU de un esquema THP reconfigurable combinado con LR. La reconfigurabilidad de las GPUs permite desactivar la etapa de LR cuando los requisitos de los usuarios están garantizados por el esquema THP, combinando complejidad computacional con rendimiento. Aunque esta implementación consigue una mejora significativa respecto a la implementación sobre CPU, su paralelismo viene limitado por la naturaleza secuencial del problema LR. Por ello, se han propuesto varias estrategias para la paralelización del problema LR que han sido evaluadas en distintas plataformas: CPU multi-núcleo, GPU y plataforma heterogénea que consiste en CPU+GPU. Los resultados revelan que la arquitectura GPU permite reducir considerablemente el tiempo de computación del problema LR, especialmente en la plataforma heterogénea.

La segunda parte de la tesis trata el problema de la realimentación de canal en sistemas FDD. En estos sistemas, los receptores normalmente proporcionan una versión cuantificada del canal a través del canal de realimentación. Con el objetivo de mantener una eficiencia alta, el canal debe ser cuantificado con los mínimos bits posibles. En primer lugar, se explora el uso de la correlación en frecuencia para reducir el volumen de información de realimentación. Se han presentado dos esquemas diferentes basados en cuantificación vectorial o VQ (vector quantization) y en la transformación Karhunen-Loève, respectivamente, y se han comparado con esquemas existentes en términos de rendimiento y complejidad computacional. Los resultados muestran que ambas técnicas son capaces de reducir significativamente el volumen de información de realimentación aprovechando la correlación en frecuencia.

Finalmente, la correlación espacial también se aprovecha para reducir la información de realimentación. Se ha presentado una caracterización espacial estadísti- 
ca del modelo de canal SCM (spatial channel model) del 3GPP (3rd Generation Partnership Project) para un entorno de alta correlación. Basado en esta caracterización, se propone un esquema de cuantificación de canal para entornos de alta correlación. Con el objetivo de obtener una caracterización para alta y baja correlación, se considera un modelo de correlación más sencillo como el modelo de Kronecker. Basado en esta caracterización, se proponen dos esquemas de cuantificación y se evalúan con un modelo de canal realista como el SCM. Los resultados muestran que ambos esquemas son capaces de reducir la información de realimentación en ambientes con correlación alta y moderada.

Palabras clave: comunicaciones inalámbricas, comunicaciones móviles, MIMO, precoding, lattice reduction, GPU, limited feedback, cuantificación de canal. 



\section{Resum}

Els sistemes de comunicacions amb múltiples antenes o sistemes MIMO (multipleinput multiple-output) es presenten com una de les tecnologies més prometedores en el camp de les comunicacions sense fils, ja que permeten aprofitar la dimensió espacial a més de les dimensions de freqüència i temps . D'aquesta manera, es poden obtenir taxes de transmissió més elevades utilitzant el mateix ample de banda, que és un recurs escàs, i mantenint una potència de transmissió baixa, la qual cosa és crucial per a dispositius alimentats per bateries. Per aquestes raons, la tecnologia MIMO ha estat adoptada en molts estàndards com LongTerm Evolution (LTE), LTE Advanced i Worldwide Interoperability for Microwave Access (WiMAX).

Les tècniques MIMO també poden es poden emprar en un escenari multiusuari, on diversos usuaris comparteixen la dimensió espacial provocant una interferència multiusuari. Mitjançant la precodificació i l'ús de múltiples antenes al transmissor, el senyal dels diferents usuaris pot ser multiplexat espacialment de manera que es mitigue la interferència multiusuari fins i tot amb usuaris d'una sola antena. Aquests sistemes, coneguts com a sistemes MU-MISO (multiuser multiple-input single-output), han atret molt l'atenció en els últims anys ja que permeten el desenvolupament de terminals petits i barats, mantenint així l'equipament més car en el transmissor.

No obstant això, aquests beneficis comporten un sistema més complex. D'una banda, el multiplexat espacial requereix una càrrega de processament considerable, que depèn de la grandària del sistema: nombre d'antenes transmissores, nombre de receptors i ample de banda. D'altra banda, les tècniques MIMO requereixen un coneixement del canal en transmissió o CSIT (channel state information at the transmitter) precís. En sistemes amb duplexació per divisió en freqüència o FDD (frequency-division duplex), la informació de canal o CSI (channel state information) ha de ser estimada en el receptor i proporcionada al transmissor a través de l'enllaç de realimentació, reduint així l'eficiència del sistema. Per tant, aquesta tesi se centra en la millora de l'eficiència de les implementacions 
de precodificació i en el rendiment dels esquemes de realimentació de canal en sistemes MU-MISO.

El problema de la precodificació s'aborda en primer lloc. S'ha dut a terme una anàlisi d'algunes de les tècniques de precodificació més usades, prestant especial atenció al seu rendiment i a la seua complexitat. Aquesta anàlisi revela que aquelles tècniques que fan ús de lattice reduction (LR) obtenen un millor rendiment. No obstant això, la complexitat computacional de la tècnica LR dificulta la seua implementació en la pràctica. L'anàlisi també revela que les tècniques zero-forcing (ZF), Tomlinson-Harashima precoding (THP) i LR-THP són les tècniques més adequades per cobrir tot el rang de rendiment i complexitat computacional. Així mateix, s'ha dut a terme una anàlisi d'aquestes tècniques sota CSIT imperfecte. Aquesta anàlisi ha demostrat que LR és una tècnica molt important també per al cas de CSIT imperfecte.

A continuació, s'han presentat implementacions paralleles de tècniques de precodificació sobre unitats de processament gràfic o GPUs (graphic processing unit), comparant-se amb implementacions en unitats de processament central o CPU (central processing unit). Atès que les implementacions de THP i LR-THP han demostrat ser les que millor s'adapten a l'arquitectura de la GPU i ja que tenen moltes operacions comunes, s'ha proposat una implementació sobre GPU d'un esquema THP reconfigurable combinat amb LR. La reconfigurabilitat de les GPUs permet desactivar l'etapa de LR quan els requisits dels usuaris estan garantits per l'esquema THP, combinant complexitat computacional amb rendiment. Encara que aquesta implementació aconsegueix una millora significativa respecte a la implementació sobre CPU, el seu paral·lelisme ve limitat per la naturalesa seqüencial del problema LR. Per això, s'han proposat diverses estratègies per a la parallelització del problema LR que han estat avaluades en diferents plataformes: CPU multi-nucli, GPU i plataforma heterogènia que consisteix en CPU+GPU. Els resultats revelen que l'arquitectura GPU permet reduir considerablement el temps de computació del problema LR, especialment a la plataforma heterogènia.

La segona part de la tesi tracta el problema de la realimentació de canal en sistemes FDD. En aquests sistemes, els receptors normalment proporcionen una versió quantificada del canal a través del canal de realimentació. Amb l'objectiu de mantenir una eficiència alta, el canal ha de ser quantificat amb els mínims bits possibles. En primer lloc, s'explora l'ús de la correlació en freqüència per reduir el volum d'informació de realimentació. S'han presentat dos esquemes diferents basats en quantificació vectorial o VQ (vector quantization) i en la transformació Karhunen-Loève, respectivament, i s'han comparat amb esquemes existents en termes de rendiment i complexitat computacional. Els resultats mostren que ambdues tècniques són capaços de reduir significativament el volum d'informació de realimentació aprofitant la correlació en freqüència. 
Finalment, la correlació espacial també s'aprofita per reduir la informació de realimentació. S'ha presentat una caracterització espacial estadística del model de canal SCM (spatial channel model) del 3GPP (3rd Generation Partnership Project) per a un entorn d'alta correlació. Basat en aquesta caracterització, es proposa un esquema de quantificació de canal per a entorns d'alta correlació. Amb l'objectiu d'obtenir una caracterització per a alta i baixa correlació, es considera un model de correlació més senzill com el model de Kronecker. Basat en aquesta caracterització, es proposen dos esquemes de quantificació i s'avaluen amb un model de canal realista com el SCM. Els resultats mostren que els dos esquemes són capaços de reduir la informació de realimentació en ambients amb correlació alta i moderada.

Paraules clau: comunicacions sense fils, comunicacions mòbils, MIMO, precoding, lattice reduction, GPU, limited feedback, quantificació de canal. 



\section{Acknowledgements}

It is a pleasure for me to thank my supervisor Dr. Gema Piñero for giving me the opportunity to pursue doctoral studies. Since the beginning, she gave me the freedom to carry out this work in my own way but, at the same time, offering me support and advice.

I am very grateful to Prof. Ana García Armada from the Universidad Carlos III de Madrid, Prof. David Gesbert from EURECOM and Prof. Rodrigo de Lamare from the University of York and the Pontifical Catholic University of Rio de Janeiro for serving as reviewers of this thesis. I am also very grateful to Prof. Ana PerezNeira from the Universitat Politècnica de Catalunya, Prof. Mikael Sternad from the Uppsala University and Dr. Vicenç Almenar from the Universitat Politècnica de València for being members of the committee. Since time is a scarce resource for everyone, I deeply appreciate the time they dedicated to review this thesis.

I would like to thank Prof. Tommy Svensson and Dr. Carmen Botella, who hosted me at the Department of Signals and Systems of Chalmers University of Technology during some months. Likewise, I would like to thank Prof. Gerhard Fettweis and Dr. Richard Fritzsche, who hosted me at Vodafone Chair Mobile Communications Systems, Technische Universität Dresden. I really appreciate the opportunity they gave me to work within their teams.

I owe my deepest gratitude to Prof. Alberto González and Prof. Antonio M. Vidal for their generous advice and help in the fields of wireless communications and computational mathematics, respectively. I would like to thank all the people at the Universitat Politècnica de València that shared my daily work at the Institute of Telecommunications and Multimedia Applications (iTEAM). In particular, I would like to thank Prof. Jose J. Lopez, Dr. María de Diego, Dr. Miguel Ferrer and Dr. Paco Martinez. Thanks also to my current and former colleagues at the iTEAM: Laura Fuster, Emanuel Aguilera, Jose A. Belloch, Jorge Lorente, Sandra Roger, Marian Simarro, Amparo Martí, Csaba M. Józsa, Eliseo Villanueva, Luis Maciá, Sandra Paredes, Carla Ramiro, Ana Torres, Toni Berenguer and Vicente Motos for all the good moments spent together not only inside but also outside the office. 
I wish also to thank the Universitat Politècnica de València for the received financial support under the FPI program.

Thanks to my group of friends in Villena. They all were able to make me break away from the daily workload. I also thank my new colleagues in Telefónica for their support and understanding.

I would also like to express my sincere gratitude to all my family for their help and encouragement. I am indebted to my cousin Carlos Maciá for his careful proofreading of this thesis. Finally, I am extremely grateful to my parents, Fernando and Cristina, to my brother Alberto and to Isa for having offered me unconditional support throughout the course of this thesis.

Fernando Domene Oltra

December 2014 


\section{Acronyms}

3GPP 3rd Generation Partnership Project

3GPP2 3rd Generation Partnership Project 2

a.k.a. also known as

ACQ adjacent conditional quantization

AoA angle of arrival

AoD angle of departure

API application programming interface

AS angular spread

ASIC application-specific integrated circuit

BC broadcast channel

BD block diagonalization

BER bit error rate

BS base station

CDI channel distribution information

CDM code-division multiplexing

CFR channel frequency response

CIR channel impulse response

CoMP coordinated multipoint

CP cyclic prefix

CPDF conditional probability density function

CPU central processing unit

CQ conditional quantization

CRAS-LLL cost-reduced all-swap LLL

CRMB-LLL cost-reduced modified block LLL 


$\begin{array}{ll}\text { CRS } & \text { cell-specific reference signal } \\ \text { CSI } & \text { channel state information } \\ \text { CSIR } & \text { channel state information at the receiver } \\ \text { CSIT } & \text { channel state information at the transmitter } \\ \text { CUDA } & \text { compute unified device architecture } \\ \text { DFT } & \text { discrete Fourier transform } \\ \text { DP } & \text { dynamic parallelism } \\ \text { DPC } & \text { dirty paper coding } \\ \text { DQ } & \text { differential quantization } \\ \text { DS } & \text { delay spread } \\ \text { e.g. } & \text { exempli gratia } \\ \text { EPA } & \text { Extended Pedestrian A } \\ \text { ETU } & \text { Extended Typical Urban } \\ \text { FDD } & \text { frequency-division duplex } \\ \text { FDM } & \text { frequency-division multiplexing } \\ \text { FFT } & \text { fast Fourier transform } \\ \text { FLOPS } & \text { floating-point operations per second } \\ \text { FPGA } & \text { field-programmable gate array } \\ \text { GBA } & \text { greedy bit allocation } \\ \text { GPGPU } & \text { general-purpose graphic processing unit } \\ \text { GPU } & \text { graphic processing unit } \\ \text { GQ } & \text { Gaussian quantization } \\ \text { GSC } & \text { Gram-Schmidt coefficient } \\ \text { GSO } & \text { Gram-Schmidt orthogonalization } \\ \text { HDSPA } & \text { high-speed downlink packet access } \\ \text { i.e. } & \text { id est } \\ \text { i.i.d. } & \text { independent and identically distributed } \\ \text { ICI } & \text { intercell interference } \\ \text { IDFT } & \text { inverse discrete Fourier transform } \\ \text { ISI } & \text { intersymbol interference } \\ \text { ITU } & \text { International Telecommunication Union } \\ \text { JP } & \text { joint processing } \\ \text { KL } & \text { Karhunen-Loève } \\ & \end{array}$


LLL Lenstra-Lenstra-Lovász

LR lattice reduction

LR-THP lattice reduction Tomlinson-Harashima precoding

LRAP lattice-reduction-aided precoding

LS least squares

LTE Long-Term Evolution

LTE-A LTE advanced

MAC multiple access channel

MB-LLL modified block LLL

MIMO multiple-input multiple-output

MISO multiple-input singular-output

MS mobile station

MSE mean square error

MU-MIMO multiuser multiple-input multiple-output

MU-MISO multiuser multiple-input singular-output

OFDM orthogonal frequency-division multiplexing

PDF probability density function

PQ polar quantization

PU2RC per user unitary rate control

QAM quadrature amplitude modulation

QoS quality of service

RAM random access memory

RB resource block

RBA real-valued bit allocation

RE resource element

RMS root mean square

RS reference signal

RVQ random vector quantization

SCM spatial channel model

SCME spatial channel model extended

SD standard deviation

SE sphere encoder 


\begin{tabular}{ll} 
SER & symbol error rate \\
SIC & successive interference cancellation \\
SIMD & single-instruction multiple-data \\
SIMT & single-instruction multiple-thread \\
SINR & signal-to-interference-plus-noise ratio \\
SISO & singular-input singular-output \\
SM & streaming multiprocessor \\
SNR & signal-to-noise ratio \\
SPMD & single program, multiple data \\
SU-MIMO & single-user multiple-input multiple-output \\
SVD & singular value decomposition \\
TB & thread-block \\
TDD & time-division duplex \\
TDM & time-division multiplexing \\
TDP & thermal design power \\
THP & Tomlinson-Harashima precoding \\
UE & user equipment \\
VB & V-BLAST, vertical Bell laboratories layered \\
VLSI & space-time \\
VP & very-large-scale integration \\
VQ & vector-perturbation \\
WiMAX & vector quantization \\
ZF & cess \\
\hline
\end{tabular}




\section{Nomenclature}

\begin{tabular}{|c|c|}
\hline$x, X$ & Scalar \\
\hline $\mathbf{x}$ & Vector \\
\hline $\mathbf{X}$ & Matrix \\
\hline$(\cdot)^{T}$ & Transpose \\
\hline$(\cdot)^{H}$ & Hermitian transpose \\
\hline$(\cdot)^{*}$ & Scalar complex conjugate \\
\hline $\operatorname{Tr}(\cdot)$ & Trace of a square matrix \\
\hline $\operatorname{det}(\cdot)$ & Determinant of a square matrix \\
\hline$(\cdot)^{-1}$ & Matrix inversion \\
\hline$(\cdot)^{\dagger}$ & Moore-Penrose pseudoinverse \\
\hline$\|\cdot\|_{F}$ & Frobenius norm of a matrix \\
\hline$\|\cdot\|$ & Euclidean norm of a vector \\
\hline$(\cdot, \cdot)$ & Inner product \\
\hline$j$ & $\sqrt{-1}$ \\
\hline$|\cdot|$ & Absolut value of a complex number \\
\hline$\angle(\cdot)$ & Phase of a complex number \\
\hline $\mathfrak{R e}\{\cdot\}$ & Real part of a complex number \\
\hline $\mathfrak{I m}\{\cdot\}$ & Imaginary part of a complex number \\
\hline $\mathbb{Z}^{m \times n}$ & Set of $m \times n$ integer matrices \\
\hline $\mathbb{R}^{m \times n}$ & Set of $m \times n$ real matrices \\
\hline $\mathbb{R}_{\geq 0}^{m \times n}$ & Set of $m \times n$ non-negative real matrices \\
\hline
\end{tabular}


$\mathbb{C}^{m \times n} \quad$ Set of $m \times n$ complex matrices

$\mathcal{C N}\left(\mu, \sigma^{2}\right) \quad$ Complex normal distribution with mean $\mu$ and variance $\sigma^{2}$

$\hat{a}=\mathcal{Q}(a) \quad$ Scalar quantization of a value

$\lceil\cdot\rceil \quad$ Ceil function (map a real number to the smallest following integer)

$\lfloor\cdot$ Floor function (map a real number to the largest previous integer)

$\lceil\cdot\rfloor \quad$ Round function (map a real number to the nearest integer)

$(\cdot)^{+} \quad \max (\cdot, 0)$

$\mathbb{E}[\cdot] \quad$ Mathematical expectation

$\operatorname{var}(\cdot) \quad$ Variance of a random variable

$\mathcal{B} \quad$ Set

$|\mathcal{B}| \quad$ Cardinality of a set

$\mathcal{L} \quad$ Lattice

$\mathbf{I}_{N} \quad$ Identity matrix of order $N$

$\operatorname{diag}\{\mathbf{x}\} \quad$ Square diagonal matrix with the elements of $\mathbf{x}$ on the main diagonal

$N_{t} \quad$ Number of antennas at the transmitter or base station

$M \quad$ Number of mobile stations

$K \quad$ Number of subcarriers 


\section{Contents}

Abstract $\quad$ iii

Resumen vii

Resum $\quad$ xi

Acknowledgements $\quad$ Xv

Acronyms $\quad$ xvii

$\begin{array}{ll}\text { Nomenclature } & \text { xxi }\end{array}$

$\begin{array}{ll}\text { Contents } & \text { xxiii }\end{array}$

List of Figures $\quad$ xxvii

List of Tables $\quad$ xxxi

1 Introduction 1

1.1 Motivation ....................... 4

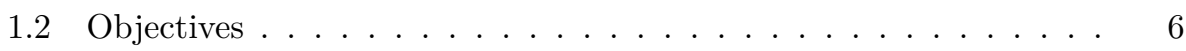

1.3 Contributions ......................... 6

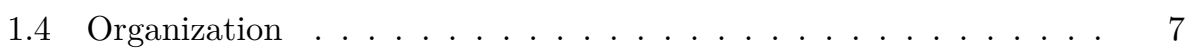

2 Preliminaries $\quad 11$

2.1 MIMO systems . . . . . . . . . . . . . . . . . . . . . 11

2.1.1 Single-user . . . . . . . . . . . . . . . . . . . . . . 12

2.1.2 Multiuser ..................... 14

2.2 Coordinated systems . . . . . . . . . . . . . . . . 19

2.3 Massive MIMO systems . . . . . . . . . . . . . . . 21 
2.4 MIMO-OFDM . . . . . . . . . . . . . . . . . . 22

2.5 Limited Feedback . . . . . . . . . . . . . . . . . . . . . . . . 24

2.5.1 Single-user . . . . . . . . . . . . . . . . 24

2.5 .2 Multiuser .................... 26

2.6 Channel models . . . . . . . . . . . . . . . . . . . . . . . . . 29

2.6.1 Gaussian i.i.d. model . . . . . . . . . . . . . . . 30

2.6.2 Kronecker model . . . . . . . . . . . . . . . . . 30

2.6.3 Extended ITU model . . . . . . . . . . . . . . . . . . . . . . . . 30

2.6.4 Spatial channel model . . . . . . . . . . . . . . 31

2.7 Lattices . . . . . . . . . . . . . . . . . . . . . . . . . . . . . . . . . . . . 33

2.7.1 Introduction . . . . . . . . . . . . . . . . 33

2.7 .2 Lattice reduction . . . . . . . . . . . . . . . 35

I Precoding $\quad 39$

3 Precoding with Perfect CSIT $\quad 41$

3.1 Zero-Forcing . . . . . . . . . . . . . . . . . . 44

3.2 Sphere encoder . . . . . . . . . . . . . . . . 45

3.3 Tomlinson-Harashima precoding . . . . . . . . . . . . . 46

3.4 Lattice-Reduction-aided precoding . . . . . . . . . . . . . . . 48

3.4.1 LRAP-linear . . . . . . . . . . . . . . . . 48

3.4 .2 LRAP-VB . . . . . . . . . . . . . . . . 49

3.4.3 LR-THP . . . . . . . . . . . . . . . 50

3.5 Performance . . . . . . . . . . . . . . . . . . 51

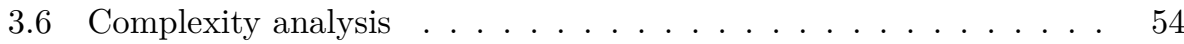

3.6.1 Preprocessing computational cost . . . . . . . . . 55

3.6.2 Per-symbol-vector computational cost . . . . . . . . . . 57

3.6.3 Overall computational cost . . . . . . . . . . 58

3.7 Conclusion . . . . . . . . . . . . . . . . 59

4 Precoding with Imperfect CSIT $\quad 61$

4.1 Zero-Forcing. . . . . . . . . . . . . . 62

4.2 Tomlinson-Harashima precoding . . . . . . . . . . . . . . 63

4.3 Lattice-Reduction-aided Tomlinson-Harashima precoding . . . . . 64

4.4 Performance..................... 65

4.5 Conclusion . . . . . . . . . . . . . . . 68

5 Hardware Implementation $\quad 69$

5.1 Introduction to GPU and CUDA . . . . . . . . . . . . 70

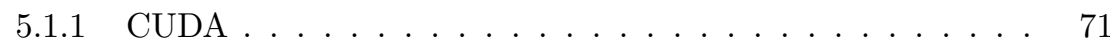


5.1.2 Hardware considerations . . . . . . . . . . . . . . 73

5.2 GPU implementation of precoding algorithms . . . . . . . . . . . 74

5.2 .1 Implementation details . . . . . . . . . . . . . . . . 74

5.2 .2 Results .................... . . 77

5.3 Reconfigurable GPU implementation of THP . . . . . . . . . . . 80

5.3.1 Reconfigurable THP scheme . . . . . . . . . . . . . . 81

5.3 .2 Implementation details . . . . . . . . . . . . . . . . . . 81

5.3 .3 Results ................... 82

5.4 Parallelization of the LLL algorithm on GPU . . . . . . . . . . 85

5.4.1 Introduction to parallel lattice reduction strategies . . . . . 85

5.4 .2 Cost-reduced all-swap LLL algorithm . . . . . . . . . . . 86

5.4 .3 Modified block LLL . . . . . . . . . . . . . . . . . . . . 89

5.4 .4 Cost-reduced modified block LLL . . . . . . . . . . . . . . 91

5.4.5 Implementation of MB-LLL and CRMB-LLL . . . . . . . . 91

5.4.6 Results ..................... 95

5.5 Conclusion . . . . . . . . . . . . . . . . . . . 101

\section{Limited Feedback 103}

6 Limited Feedback exploiting Frequency Correlation 105

6.1 Introduction to quantization . . . . . . . . . . . . . 106

6.1.1 Scalar quantization . . . . . . . . . . . . 106

6.1 .2 Vector quantization ................ 113

6.2 Vector quantization over CFR pilots . . . . . . . . . . . . . 117

6.2.1 Channel estimation . . . . . . . . . . . . . . 118

6.2.2 Vector quantization . . . . . . . . . . . . . . . 119

6.2 .3 Numerical results . . . . . . . . . . . . . . . . . . . 121

6.2.4 Conclusion . . . . . . . . . . . . . . 124

6.3 Channel quantization based on the KL transform . . . . . . . . . . 124

6.3.1 Time-domain quantization . . . . . . . . . . . . . 125

6.3.2 Karhunen-Loève-Domain Quantization . . . . . . . . . 126

6.3.3 Efficient eigendecomposition . . . . . . . . . . . . 128

6.3.4 Computational complexity . . . . . . . . . . . . . 129

6.3.5 Numerical results . . . . . . . . . . . . . . . . . 130

6.3.6 Conclusion .................... 134

6.4 Conclusion . . . . . . . . . . . . . . . . . . . . 134

7 Limited Feedback exploiting Spatial Correlation 135

7.1 Quantization based on the spatial characterization of the SCM . . 136

7.1.1 System model . . . . . . . . . . . . . . . 136

7.1.2 Statistical characterization of the spatial channel . . . . . 137 
7.1.3 Proposed quantization scheme . . . . . . . . . . . 140

7.1 .4 Extension to CoMP . . . . . . . . . . . . . . 141

7.1 .5 Numerical results . . . . . . . . . . . . . . . . . 143

7.1 .6 Conclusion ... . . . . . . . . . . . . 150

7.2 Quantization based on the spatial characterization of the Kronecker

correlation model . . . . . . . . . . . . . . . . 151

7.2.1 System model . . . . . . . . . . . . . . . . . 152

7.2.2 Statistical characterization of the spatial channel . . . . . . 153

7.2.3 Proposed quantization scheme . . . . . . . . . . . 158

7.2.4 Alternative quantization scheme . . . . . . . . . . . 167

7.2 .5 Numerical results . . . . . . . . . . . . . . . . . . . . . . 168

7.2 .6 Conclusion ..................... 173

7.3 Conclusion . . . . . . . . . . . . . . . . 175

8 Conclusions $\quad 177$

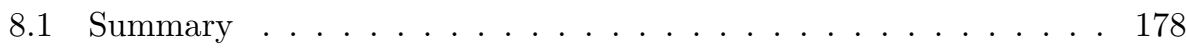

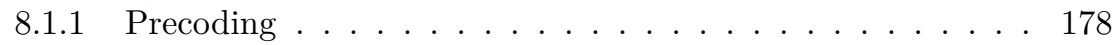

8.1 .2 Limited feedback . . . . . . . . . . . . . . . 179

8.2 Future work . . . . . . . . . . . . . . . 180

8.3 Publications . . . . . . . . . . . . . . . . 181

$\begin{array}{ll}\text { Appendices } & 183\end{array}$

A CRAS-LLL: CUDA and OpenMP pseudocode 185

B Conditional quantization codebook: details of calculations 189

$\begin{array}{lc}\text { Bibliography } & 193\end{array}$ 


\section{List of Figures}

1.1 Expected evolution of global mobile traffic per month . . . . . . . 2

1.2 Expected evolution of mobile data traffic per application . . . . . . 2

1.3 Expected evolution of mobile subscriptions . . . . . . . . . . 3

2.1 SU-MIMO system model . . . . . . . . . . . . . . . . . . . . . . . . . . 12

2.2 MU-MIMO system . . . . . . . . . . . . . . . . . . . . . . . . . 15

2.3 Uplink of a MU-MIMO system . . . . . . . . . . . . . . . . . . . . 16

2.4 Capacity region of the MIMO MAC . . . . . . . . . . . . . 17

2.5 Downlink of a MU-MIMO system . . . . . . . . . . . . . . . . . . 18

2.6 Dirty paper region . . . . . . . . . . . . . . . . . . . . . . . 19

2.7 Example of a coordinated cluster . . . . . . . . . . . . . . . . . 20

2.8 Massive MIMO base station . . . . . . . . . . . . . . . . . . . 21

2.9 Angular parameters in SCM specifications . . . . . . . . . . . . 32

2.10 Example lattices showing their fundamental parallelotope . . . . . 35

2.11 Example lattices showing their Voronoi region . . . . . . . . . . 35

3.1 Effect of the perturbation in square $\mathcal{M}$-QAM modulated signals . 43

3.2 ZF precoding scheme . . . . . . . . . . . . . . . . . 44

3.3 SE scheme ............................ 46

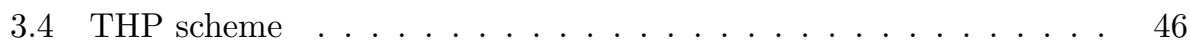

3.5 LRAP-linear scheme . . . . . . . . . . . . . . . . . . . . . . . . . . . . . . 49

3.6 LRAP-VB scheme . . . . . . . . . . . . . . . . . 50

3.7 LR-THP scheme . . . . . . . . . . . . . . . . . 51

3.8 BER for the different precoding techniques for $N_{t}=M=4 \ldots$. . $\quad 52$

3.9 BER for the different precoding techniques for $N_{t}=M=8$. . . $\quad 53$

3.10 Sum-rate for the different precoding techniques for $N_{t}=M=4$. . 54

3.11 Sum-rate for the different precoding techniques for $N_{t}=M=8$. . $\quad 55$

3.12 Arithmetic operations of the precoding algorithms for $N_{t}=4$. . $\quad 59$

4.1 BER for $N_{t}=M=4$ and $\sigma_{e}^{2}=0,10^{-2}, 10^{-3} \ldots \ldots$. . . . . 65

4.2 BER for $N_{t}=M=8$ and $\sigma_{e}^{2}=0,10^{-2}, 10^{-3} \ldots \ldots 6$

4.3 Sum-rate for $N_{t}=M=4$ and $\sigma_{e}^{2}=0,10^{-2}, 10^{-3} \ldots \ldots$. . . . . 67

4.4 Sum-rate for $N_{t}=M=8$ and $\sigma_{e}^{2}=0,10^{-2}, 10^{-3} \ldots \ldots$. . . . 68 
5.1 Example of execution of a CUDA program . . . . . . . . . . . 72

5.2 Host memory and device memory . . . . . . . . . . . . . . . . 73

5.3 Speed-up for $N_{t}=M=4$ and different values of $K \ldots \ldots$. . . . 78

5.4 Speed-up per stage for $N_{t}=M=4$ and different values of $K$. . . 79

5.5 Computational time in GPU . . . . . . . . . . . . . . 80

5.6 Reconfigurable THP scheme . . . . . . . . . . . . . . . . . . 81

5.7 Speed-up of THP and LR-THP . . . . . . . . . . . . . . . . . . . 83

5.8 Computational time of THP and LR-THP . . . . . . . . . . . . . . 84

5.9 CRAS-LLL algorithm mapping to GPU architecture . . . . . . . . 89

5.10 Kernels scheduling for the MB-LLL algorithm . . . . . . . . . . . . 92

5.11 Kernels scheduling on the heterogeneous platform for the MB-LLL algorithm . . . . . . . . . . . . . . . 95

5.12 Computational time of the CRAS-LLL algorithm on different GPUs 97

5.13 Computational time of the CRAS-LLL and MB-LLL algorithms . . 98

5.14 Computational time of the MB-LLL algorithm on different archi-

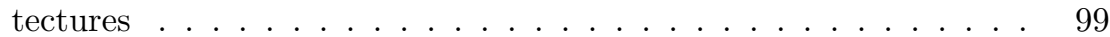

5.15 Computational times of CRAS-LLL, MB-LLL and CRMB-LLL algorithms on different architectures . . . . . . . . . . . . 100

6.1 Example of uniform quantizer . . . . . . . . . . . . . . . 109

6.2 Lloyd algorithm for quantizer design . . . . . . . . . . . . . . . 110

6.3 Scalar quantization vs. vector quantization . . . . . . . . . . . . 114

6.4 Lloyd algorithm for quantizer design with empirical data . . . . . . 116

6.5 System model . . . . . . . . . . . . . . . . . . . 117

6.6 Pilot symbol distribution for channel estimation . . . . . . . . . . . 119

6.7 Average MSE for the quantized channel . . . . . . . . . . . . . . . 122

6.8 BER for $L_{\mathrm{v}}=16$ and different values of $B_{\mathrm{v}} \ldots \ldots$. . . . . . . . . 123

6.9 Quantized channel using time-domain and KL quantization schemes 131

6.10 MSE for EPA and ETU channel models and different number of modulated subcarriers . . . . . . . . . . . . . . . 132

6.11 MSE for EPA and ETU channel models for different number of

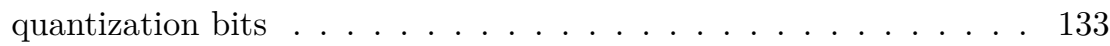

7.1 Example of a $120^{\circ}$ sector . . . . . . . . . . . . . . . . 137

7.2 Estimated PDF of $\left|h_{n s} / h_{n^{\prime} s}\right|$ and its Laplacian approximation for $R=20$ subpaths and reference antenna $n^{\prime}=2 \ldots \ldots 138$

7.3 Estimated PDF of the different CIR parameters . . . . . . . . . . 139

7.4 Example of a coordinated cluster . . . . . . . . . . . . . . . 141

7.5 Proposed pilot symbol allocation for a coordinated cluster . . . . . 142

7.6 MSE vs. cost for GQ and DQ feedback schemes . . . . . . . . . . . 146

7.7 Average uncoded BER per subcarrier for ZF, THP and LR-THP with GQ and DQ feedback schemes and SNR=30 dB. . . . . . . . 147

7.8 Average sum-rate per subcarrier for ZF, THP and LR-THP with GQ, DQ and Perfect CSIT and $\mathrm{SNR}=30 \mathrm{~dB}$. . . . . . . . . 148 
7.9 Average uncoded BER per subcarrier for ZF, THP and LR-THP with GQ $\left(B_{G}=7\right)$, DQ $\left(B_{R}=7, B_{M}=6, B_{P}=8\right)$ and Perfect CSIT . . . . . . . . . . . . . . . . . . 149

7.10 Average sum-rate per subcarrier for ZF, THP and LR-THP with GQ $\left(B_{G}=7\right)$, DQ $\left(B_{R}=7, B_{M}=6, B_{P}=8\right)$ and Perfect CSIT. . 150

7.11 CPDF of (7.26) for different envelope values of $r_{\mathrm{r}}$ and a fixed value of $|\rho|=0.9 \ldots \ldots \ldots \ldots \ldots \ldots \ldots$

7.12 CPDF of (7.26) for a fixed envelope value of $r_{\mathrm{r}}=2$ and different values of $|\rho| \ldots \ldots \ldots \ldots$. . . . . . . . . . . . . . . . . . . . . 156

7.13 PDF of $\Delta$ in (7.32) for different values of $|\rho| \ldots \ldots . . . . . .158$

7.14 Illustrative example of a non-reference codebook . . . . . . . . . . 162

7.15 Analytical PDF of the phase deviation and its approximation using the Laplace distribution for different values of $\left|\rho_{n}\right| \ldots \ldots$. . . . . 164

7.16 Comparison between CQ and ACQ . . . . . . . . . . . . 168

7.17 MSE for $N_{t}=4, d=\lambda / 2$ and different average number of quantization bits . . . . . . . . . . . . . . 170

7.18 MSE for $N_{t}=4, d=\lambda$ and different average number of quantization bits . . . . . . . . . . . . . . . . 172

7.19 MSE for $N_{t}=8, d=\lambda / 2$ and different average number of quantization bits . . . . . . . . . . . . . . . 173

7.20 MSE for $N_{t}=4$, different antenna separations and an average number of quantization bits of 28 bits . . . . . . . . . . . . . . . 174

7.21 MSE for $N_{t}=8$, different antenna separations and an average number of quantization bits of 56 bits . . . . . . . . . . . . . 174 



\section{List of Tables}

2.1 Power delay profiles of extended ITU models . . . . . . . . . . . . 31

2.2 Environment parameters in SCM specifications . . . . . . . . . . . 33

3.1 Main preprocessing stages of precoding algorithms . . . . . . . . 56

3.2 Additional preprocessing cost of precoding algorithms . . . . . . 57

3.3 Per-symbol-vector cost of precoding algorithms . . . . . . . . . . 57

3.4 Minimum value of $L_{\mathrm{ch}}$ for $C_{\mathrm{psv}}>\left(C_{\mathrm{pre}} / L_{\mathrm{ch}}\right) \ldots \ldots$. . . . . 58

$5.1 \mathrm{ZF}$ and $\mathrm{THP}$ algorithms ................... 75

5.2 LRAP algorithms . . . . . . . . . . . . . . . . . 76

5.3 Nvidia Quadro FX 5800 GP features . . . . . . . . . . . . . . . 77

5.4 Nvidia Tesla C2070 features . . . . . . . . . . . . . . . . . . 82

5.5 Nvidia GTX690, K20 and C2075 GPU features . . . . . . . . . . 96

5.6 Performance comparison of different LR implementations . . . . . 102

6.1 Comparison of the computational complexity . . . . . . . . . 129

7.1 DQ feedback scheme . . . . . . . . . . . . . . . . 140

7.2 Channel and system parameters . . . . . . . . . . . . . . 143

7.3 Configurations of the Differential Quantizer. . . . . . . . . . . . . 145

7.4 Proposed quantization scheme . . . . . . . . . . . . 160 



\section{Chapter 1}

\section{Introduction}

This chapter presents a brief introduction to wireless and mobile communications and also describes the motivation of this thesis. Next, the objectives and contributions of the thesis are presented and, finally, the organization of this dissertation is described.

Wireless communications and particularly, mobile communications, are currently experiencing an exceptional growth and development. The appearance of smartphones and tablets, the applications and services enabled by these devices and the possibility of having Internet access everywhere have motivated this growth. Some interesting statements and predictions supporting this growth can be found in $[1-3]$ :

- The mobile data traffic in 2013 was nearly 18 times the size of the entire global Internet in 2000, and it is expected to grow at a compound annual growth rate of around 50\% in 2014-2019 (45\% and 61\% according to [1] and [2], respectively). As seen in Figs. 1.1 and 1.2, this is mainly due to mobile traffic generated by the smartphones and new high data-consuming services such as video.

- Global mobile devices and connections reached 7 billion in 2013 and, by the end of 2014, it is expected that the number of mobile-connected devices will exceed the number of people on earth. In addition, as seen in Fig. 1.3, the number of LTE subscribers is expected to reach 2.6 billion by 2019, representing around $30 \%$ of total mobile subscriptions.

- In average, a $4 \mathrm{G}$ connection generated 14.5 times more traffic than a non-4G connection in 2013. Thus, the increase in the number of $4 \mathrm{G}$ users translates into a large growth in the mobile data traffic. 
Global mobile traffic (monthly ExaBytes)

20

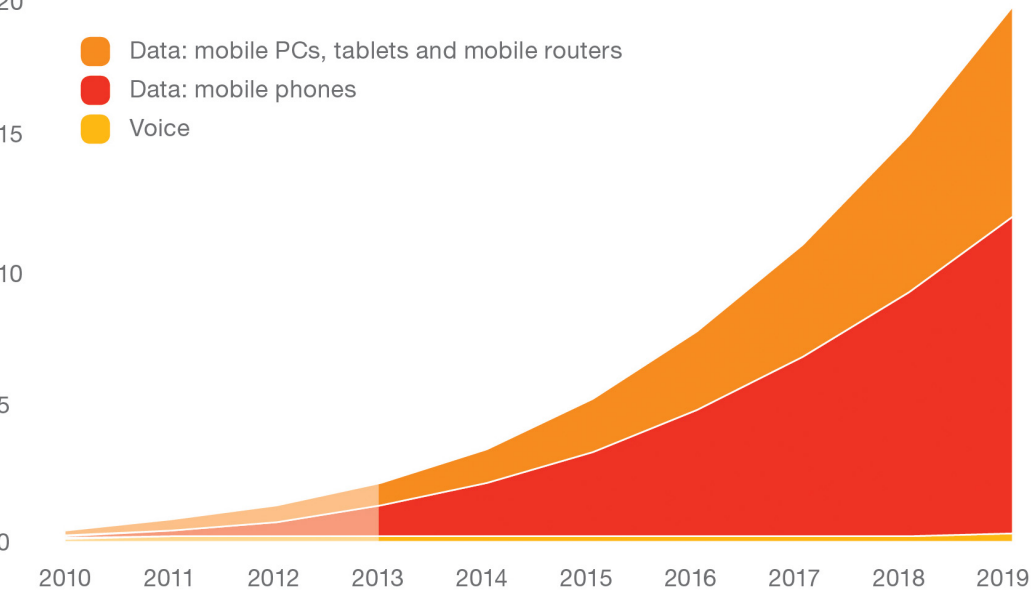

Figure 1.1: Expected evolution of global mobile traffic per month [1].

Mobile data traffic by application type (monthly ExaBytes)

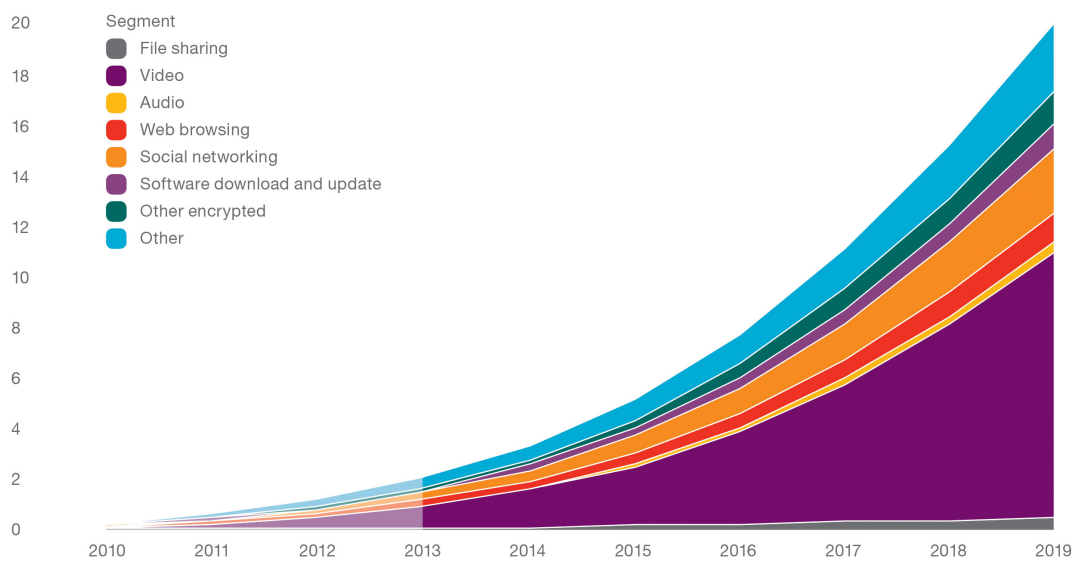

Figure 1.2: Expected evolution of mobile data traffic per application [1]. 


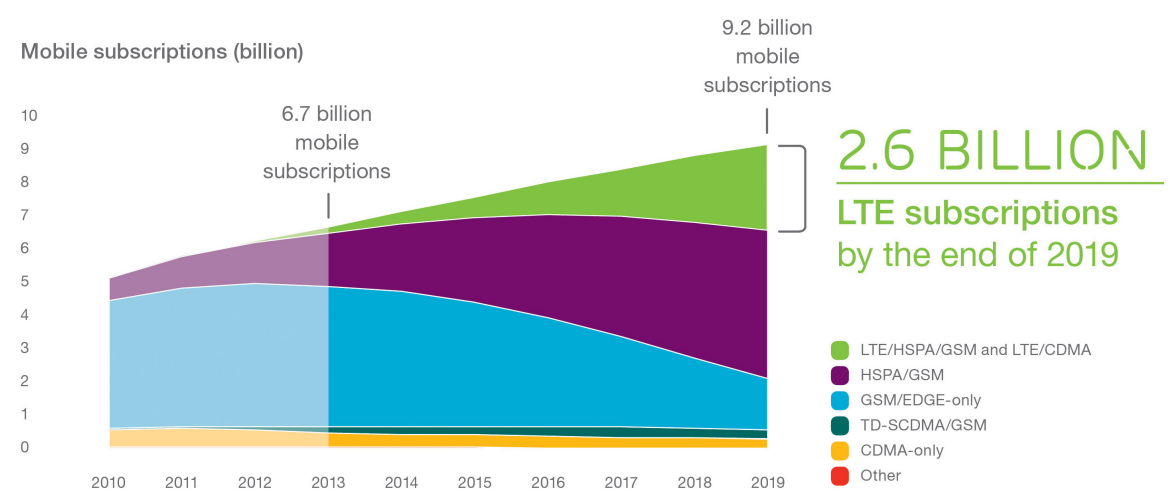

Figure 1.3: Expected evolution of mobile subscriptions [1].

Due to the increase of the data traffic and the scarcity of other resources such as spectrum or transmit power, two of the main requirements for modern and future wireless communication systems are high spectral and energy efficiencies. MIMO communication systems have emerged as one of the most promising technologies $[4,5]$. In these systems, the transmitter and the receiver are equipped with several antennas. MIMO techniques allow to leverage the spatial dimension in addition to the time and frequency dimensions, which are fully utilized in traditional communication systems. Thus, higher rates can be obtained by using the same bandwidth and by keeping a low transmit power, which is crucial in battery-operated devices.

MIMO techniques can also be used in a multiuser scenario, such as in cellular systems. This case is slightly different since several users are sharing the spatial dimension, causing multiuser interference [6]. However, thanks to the multiple antennas at the transmitter and the use of precoding, the multiuser interference can be mitigated even when the users are equipped with only one antenna. Thus, precoding allows the spatial separation of different users that share the same time and frequency resources.

Orthogonal frequency-division multiplexing (OFDM) has also become a very attractive technique in wireless communications during the last decades. OFDM is used to mitigate the effects of intersymbol interference (ISI) in frequency selective channels, turning a broadband frequency selective channel into a set of parallel narrowband frequency flat subchannels [7]. A high spectral efficiency is also achieved since the frequency separation between subcarriers is the minimum required to preserve the orthogonality. MIMO techniques can enhance the performance of OFDM by exploiting the spatial domain. OFDM simplifies the signal processing in MIMO techniques, since it is performed over narrowband flat-fading channels 
and each channel can be processed independently. This combination, known as MIMO-OFDM, has been adopted by many standards such as IEEE $802.11 \mathrm{n} / \mathrm{ac}$, WiMAX, LTE, LTE-A and, presumably, 5G [8-12].

However, these benefits come at the expense of a more complex system. On the one hand, the use of the spatial dimension entails some extra processing, which can be considerable depending on the number of antennas and OFDM subcarriers [8]. In addition, MIMO techniques usually require an accurate CSIT [6]. In time-division duplex (TDD) systems, the transmitter can easily obtain this information using the reciprocal properties of the channel. However, in FDD systems, CSI has to be estimated at the receiver and provided to the transmitter through the feedback channel, hence reducing the efficiency of the system [13].

\subsection{Motivation}

As seen in the previous section, precoding techniques in multiuser multiple-input multiple-output (MU-MIMO) systems allow spatial multiplexing without requiring multiple antennas at the receivers. This fact has motivated the use of MU-MIMO techniques in many communication standards, since it allows to limit the most expensive hardware to the transmitter and the development of small and inexpensive terminals [6]. For this reason, this thesis focuses on multiuser systems with single-antenna receivers, a.k.a. MU-MISO systems. However, the addition of the spatial dimension introduces a new signal processing stage whose complexity depends on the system size, i.e., the number of transmit antennas, the number of receivers and the bandwidth or number of subcarriers.

Different precoding techniques for MU-MIMO systems have been proposed that vary in performance and computational complexity. Dirty paper coding (DPC) is a theoretical precoding technique that allows the cancellation of the interference without incurring a power penalty. However, its high complexity restricts its implementation in practical systems and suboptimal techniques have to be used. The main suboptimal techniques are ZF, THP and other non-linear techniques that make use of LR. Given a basis, LR essentially consists in find another basis whose vectors are more orthogonal and shorter according to the Euclidean norm. Although LR can involve the solution of an NP problem, a polynomial-time algorithm can be found by relaxing some conditions [14]. LR-based techniques have demonstrated to achieve the best performance among the various suboptimal precoding techniques $[15,16]$. However, their practical implementation is not easy because of the LR computational complexity.

Due to the growth experienced by data traffic in wireless communication systems, the bandwidth offered by the latest standards has increased. For instance, bandwidths of up to $100 \mathrm{MHz}$ can be used in LTE-A. The use of OFDM in MU-MISO 
systems allows to turn a broadband channel into a set of parallel narrowband channels where the MU-MISO techniques are performed independently. Hence, the complexity of this system is lower than it would be in a broadband system. However, the complexity of MU-MISO-OFDM systems is still considerably higher compared to traditional singular-input singular-output (SISO) systems.

In MU-MISO-OFDM, precoding has to be performed for every OFDM subcarrier in a limited time. In order to achieve real-time communication, the available time to perform the precoding operations corresponds approximately to the duration of an OFDM symbol. In LTE-A, the duration of an OFDM symbol is around $70 \mu \mathrm{s}$ and up to 6000 subcarriers are supported. Taking into account these values, up to 6000 precoding processes have to be performed in $70 \mu \mathrm{s}$. Thus, very efficient implementations of precoding techniques are necessary to fulfill this requirement.

Motivated by a similar problem involving detection in MIMO-OFDM systems, many-core architectures such as GPUs have been proposed for the efficient implementation of signal processing algorithms [17]. These architectures contain hundreds of processor cores and can accelerate the computation through the parallelization of the different operations $[18,19]$. Thus, the implementation of precoding algorithm on GPUs can help fulfill the aforementioned time requirements of MU-MISO-OFDM systems.

Apart from the extra processing, another disadvantage of MU-MIMO systems is the requirement of an accurate CSIT [6]. This thesis is focused on FDD systems, where a quantized version of the channel information is usually provided by the receivers through the feedback link. In order to keep a high efficiency, the feedback information has to be provided using as few bits as possible. Thus, designing feedback schemes to reduce the amount of feedback information is a key aspect for future wireless communication systems [13].

Time, frequency and spatial correlation are usually leveraged to reduce the amount of feedback information. Feedback schemes which take advantage of the time and frequency correlation can be considered as extensions of the feedback schemes in SISO systems to the MIMO case. These schemes perform in a per-antenna manner, ignoring the spatial domain that usually shows a considerable correlation. On the other hand, feedback schemes that consider the spatial correlation can be considered specific to MIMO systems. These schemes deal with the spatial correlation as an additional resource along with time and frequency correlation in order to reduce the feedback overhead. 


\subsection{Objectives}

The objectives of this thesis are mainly focused on improving the efficiency of precoding implementations and the performance of feedback schemes in MU-MISOOFDM systems. Particularly, the main objectives of this thesis are:

- Evaluation of the performance and computational complexity of the existing precoding techniques, including a fair comparison among them.

- Efficient implementation of the existing precoding techniques on different parallel architectures.

- Development of new channel quantization schemes that contribute to the reduction of the feedback information by taking advantage of the frequency and spatial correlations.

\subsection{Contributions}

The main contributions of the thesis are:

Comparison of the performance and computational complexity of the existing precoding techniques

A comparison of the performance and computational complexity of some of the most used precoding techniques, such as ZF [20], THP [21], sphere encoder (SE) [22] and LR-based precoding techniques [15, 16], has been carried out in a unified framework. The computational complexity analysis has separated the different operations into two types: channel-related operations and signal-related operations. This separation allows the evaluation of the computational complexity independently of the channel characteristics.

\section{Efficient implementation of the existing precoding techniques}

A parallel GPU implementation of precoding techniques in MU-MISOOFDM systems has been proposed. The GPU implementation has been compared with its CPU counterpart, achieving significant speed-ups. In addition, the LR stage, which has shown to be the main bottleneck in the GPU implementations, has been addressed independently. The implementation of LR on a heterogeneous platform consisting of CPU+GPU has been proposed and compared to implementations of CPU and GPU, demonstrating that it achieves a significant improvement. 


\section{Design of channel quantization schemes leveraging the frequency cor- relation}

Two channel quantization schemes that take advantage of the frequency correlation to reduce the feedback overhead have been proposed. The first scheme is based on VQ. The performance achieved by different configurations of the quantizer has been evaluated. Results show an interesting trade-off between performance and computational complexity. The second scheme is based on scalar quantization. The scheme consists of using the $\mathrm{KL}$ transform to decorrelate the channel information in the frequency domain before quantizing it. This scheme has been compared with a scheme based on time-domain channel quantization, showing a better performance in realistic scenarios.

\section{Design of channel quantization schemes leveraging the spatial correla- tion}

Three channel quantization schemes that take advantage of the spatial correlation to reduce the feedback information have been proposed. All schemes have been evaluated in a realistic channel model such as the SCM from the 3GPP. The first scheme is based on a spatial statistical characterization of the SCM itself. Due to the complexity of this model and the large number of parameters involved, the statistical characterization has been obtained only for the high correlation case. The proposed quantization scheme has shown to successfully reduce the feedback overhead in highly correlated environments.

In order to obtain a more complete spatial statistical characterization, the Kronecker correlation model has been considered. This simpler model allows the characterization for high and low correlations. Based on this characterization, two channel quantization schemes have been presented and evaluated using the SCM. Results show that the proposed schemes outperform some existing schemes in highly and moderately correlated environments.

\subsection{Organization}

First, this thesis presents an introduction to MIMO systems and some other useful concepts as preliminaries. Next, the dissertation is organized in two parts: precoding and limited feedback. The chapters are organized as follows:

Chapter 2 This chapter contains an introduction to MIMO systems. Particularly, single-user and multiuser MIMO systems, coordinated systems and massive MIMO systems are analyzed. Additionally, an introduction to MIMOOFDM is presented. Next, a review is given of the main concepts and 
schemes in the topic of limited feedback in wireless communication. Finally, the channel models used throughout this dissertation are described and a brief overview of lattices is also provided.

Part I This part contains the chapters related to precoding.

Chapter 3 This chapter contains the description of different precoding techniques (ZF, SE, THP, LRAP-linear, LRAP-VB and LR-THP) assuming a perfect CSIT. Their performance in terms of bit error rate (BER) and sum-rate, as well as their computational complexity are analyzed and compared.

Chapter 4 In this chapter, a comparison among the performance of ZF, THP, and LR-THP is carried out when imperfect CSIT is available. Again, the performance is shown in terms of BER and sum-rate.

Chapter 5 This chapter presents different implementations of the precoding algorithms presented in previous chapters. First, a brief introduction to GPUs as general computing devices is provided. Next, the different precoding algorithms are implemented on a GPU for a MUMISO-OFDM system, and the implementations of THP and LR-THP are optimized and joined in a reconfigurable implementation. Finally, the problem of LR is addressed independently and several parallel implementations are proposed.

Part II This part contains the chapters related to limited feedback.

Chapter 6 This chapter contains a general introduction to scalar and VQ. Next, two different channel quantization schemes that allow leveraging the frequency correlation to reduce the feedback overhead are presented. The first scheme is based on VQ. The performance achieved by different configurations of the quantizer are evaluated. The second scheme is based on scalar quantization, and its performance is compared with other existing techniques.

Chapter 7 This chapter contains the spatial statistical characterization of the SCM and the Kronecker correlation model. Based on these characterizations, three channel quantization schemes have been presented and evaluated considering the SCM. The scheme based on the characterization of the SCM has shown to outperform existing schemes in a highly correlated environment, whereas the schemes based on the Kronecker correlation have shown to outperform existing schemes for both low and high correlation. 
Chapter 8 Finally, the conclusions obtained throughout the thesis and some suggestions for future research are presented. In addition, a list of the publications related to this thesis is also included. 



\section{Chapter 2}

\section{Preliminaries}

This chapter contains a brief overview of MIMO communication systems, from the well-studied single-user MIMO case to the massive MIMO system, which is the newest research field in MIMO communications. The different MIMO systems are presented throughout the different sections of this chapter. Next, a review of different feedback schemes and channel models for MIMO systems are presented. Finally, the concept of lattices and the problem of lattice reduction is introduced in the last section.

\section{$2.1 \quad$ MIMO systems}

MIMO systems have been the subject of extensive research in the last decades due to the promising performance that they offer. In systems that are power or bandwidth limited, MIMO techniques increase the system robustness or the throughput by using multiple transmit and receive antennas [23, 24]. The main concept in MIMO communication is to introduce the signal processing in the spatial domain at both the transmitter and the receiver. Thus, the signal can be combined to improve the reliability of the communication by adding diversity or by increasing the data rate through spatial multiplexing. In addition, these systems benefit from a multipath environment, which traditionally has been one of the main limitations of wireless communication systems [25]. However, MIMO systems have a higher complexity and require more hardware resources than SISO systems. 


\subsubsection{Single-user}

A single-user multiple-input multiple-output (SU-MIMO) system consists of a transmitter equipped with $N_{t}$ antennas and a receiver equipped with $N_{r}$ antennas, as can be seen in Fig. 2.1. In SU-MIMO systems, the channel is modeled as a matrix that contains the propagation effects from every transmit antenna to every receive antenna. The received signal in a narrowband SU-MIMO system can be expressed as

$$
\mathbf{y}=\mathbf{H x}+\mathbf{n},
$$

where vector $\mathbf{y} \in \mathbb{C}^{N_{r} \times 1}$ contains the received signal at every receive antenna, the element $h_{i j}$ of matrix $\mathbf{H} \in \mathbb{C}^{N_{r} \times N_{t}}$ represents the channel gain from the $j$ th transmit antenna to the $i$ th receive antenna, vector $\mathbf{x} \in \mathbb{C}^{N_{t} \times 1}$ represents the transmitted signal and vector $\mathbf{n} \in \mathbb{C}^{N_{r} \times 1}$ is the noise vector whose elements are independent and identically distributed (i.i.d.) circularly-symmetric complex Gaussian variables, with zero mean and unit variance, $\mathcal{C N}(0,1)$. The transmitter is assumed to be power limited with an average power constraint across all the antennas, $\mathbb{E}\left[\mathbf{x}^{H} \mathbf{x}\right] \leq P[26]$. Since the noise power is normalized, the power of the transmitted signal is commonly referred as the signal-to-noise ratio (SNR).

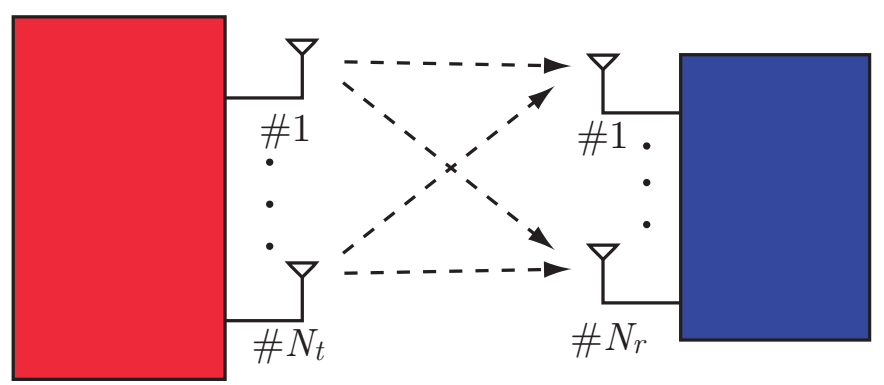

Figure 2.1: SU-MIMO system model.

The performance of SU-MIMO systems considerably depends on the knowledge of the channel matrix that the transmitter and receiver have. Different situations according to the amount of CSI are usually analyzed. The ideal case takes place when both transmitter and receiver have perfect CSI. For static or low mobility channels, an accurate channel state information at the receiver (CSIR) can be obtained through channel estimation using pilot signals. More details on channel estimation techniques in modern communication systems can be found in [11, Chap. 8] and references therein. In general, CSIT is harder to obtain. In TDD systems, the transmitter can obtain a partial CSIT using the reciprocal properties of the channel. However, in FDD systems, CSIT has to be provided by the receiver through a feedback channel. In high mobility environments, other assumptions including a statistical knowledge of the channel or channel distribution information (CDI) are used. For simplicity, in this chapter we examine the capacity of a SU- 
MIMO system assuming a perfect CSI. A more detailed analysis can be found in [26] and [27, Chap. 2].

For constant channels and assuming perfect CSIT and CSIR, the capacity of a SU-MIMO system (expressed in bps/Hz) can be expressed as:

$$
C=\max _{\mathbf{Q}: \operatorname{Tr}(\mathbf{Q})=P} \log _{2} \operatorname{det}\left(\mathbf{I}+\mathbf{H} \mathbf{Q} \mathbf{H}^{\dagger}\right),
$$

where $\mathbf{Q}=\mathbb{E}\left[\mathbf{x x}^{H}\right]$ is the covariance matrix of the transmitted signal and the optimization is performed over it [24].

The SU-MIMO channel can be converted into $\min \left(N_{t}, N_{r}\right)$ parallel and non-interfering SISO channels through the singular value decomposition (SVD) of the channel matrix [24]. The SVD of matrix $\mathbf{H}$ is a factorization of the form

$$
\mathbf{H}=\mathbf{U} \boldsymbol{\Sigma} \mathbf{V}^{H},
$$

where $\mathbf{U} \in \mathbb{C}^{N_{r} \times N_{r}}$ and $\mathbf{V} \in \mathbb{C}^{N_{t} \times N_{t}}$ are unitary matrices and $\boldsymbol{\Sigma} \in \mathbb{R}_{\geq 0}^{N_{r} \times N_{t}}$ is a diagonal matrix that contains the singular values $\sigma_{i}$ of $\mathbf{H}$. There are $R_{\mathbf{H}}$ non-zero singular values, where $R_{\mathbf{H}} \leq \min \left(N_{t}, N_{r}\right)$ is the rank of $\mathbf{H}$. Pre-multiplying the original signal by $\mathbf{V}$, i.e. transmit precoding, and post-multiplying the received signal by $\mathbf{U}^{H}$, i.e. receiver shaping, (2.1) results in

$$
\tilde{\mathbf{y}}=\boldsymbol{\Sigma} \tilde{\mathbf{x}}+\tilde{\mathbf{n}},
$$

where $\tilde{\mathbf{y}}=\mathbf{U}^{H} \mathbf{y}, \tilde{\mathbf{x}}=\mathbf{V}^{H} \mathbf{x}$ and $\tilde{\mathbf{n}}=\mathbf{U}^{H} \mathbf{n}$. It is important to note that $\mathbf{n}$ and $\tilde{\mathbf{n}}$ have the same distribution since $\mathbf{U}$ is unitary. As $\boldsymbol{\Sigma}$ is a diagonal matrix with $R_{\mathbf{H}}$ positive elements, (2.4) describes $R_{\mathbf{H}}$ parallel and non-interfering SISO channels. Since the different SISO channels may have different gains, $\sigma_{i}$, the power allocation over the different channels can be adjusted to optimize the system rate. The optimal power allocation of the $i$ th parallel channel can be calculated as

$$
P_{i}=\left(\mu-\frac{1}{\sigma_{i}^{2}}\right)^{+}, \quad 1 \leq i \leq R_{\mathbf{H}},
$$

where $\mu$ is the solution to $\sum_{i=1}^{R_{\mathbf{H}}} P_{i}=P$, which can be obtained with the waterfilling algorithm $[24,28]$. Thus, the capacity of the MIMO channel can be expressed as

$$
C=\sum_{i=1}^{R_{\mathbf{H}}}\left(\log _{2}\left(\mu \sigma_{i}^{2}\right)\right)^{+},
$$

which is equivalent to (2.2) with the optimal covariance matrix obtained as $\mathbf{Q}=$ $\mathbf{V P V} \mathbf{V}^{H}$, where $\mathbf{P}$ is a diagonal matrix with the values $P_{i}$ in its diagonal [27]. The capacity obtained in (2.6) is a theoretical bound and can require techniques that are difficult to implement in real systems due to their high complexity. In practice, techniques with a lower complexity are used. 
SU-MIMO techniques can be divided in two categories: diversity techniques and multiplexing techniques [4]. Diversity techniques consist of transmitting the signal over independent fading paths in time, frequency or space. In MIMO communications, spatial diversity is used since it does not require additional transmission time or bandwith. In the MIMO channel, up to $N_{t} N_{r}$ links with independent fading can be found. A carefully design of the transmitted signal and a suitable combination of the received signal allow the reduction of the amplitude variability of the resultant signal compared to a SISO link [5]. This technique is known as space-time coding [29, 30].

As stated before, SU-MIMO systems can achieve an increase of up to $\min \left(N_{t}, N_{r}\right)$ in the capacity compared with SISO systems. This improvement is achieved through spatial multiplexing of up to $\min \left(N_{t}, N_{r}\right)$ streams. Spatial multiplexing can be used even without CSIT. In this case, the transmitter sends independent signals through each one of the transmit antennas and the receiver has to determine the original signal despite the interference introduced by the MIMO channel. There are many techniques to do so, which differs in performance and complexity [31-33].

SU-MIMO techniques can increase the reliability of the communication (through spatial diversity) and the data rate (through spatial multiplexing). Thus, the trade-off between spatial multiplexing and diversity in each of the spatial streams plays an important role in MIMO communication systems and it has been analyzed in [34], [35, Chap. 9] and [36, Chap. 8].

\subsubsection{Multiuser}

A MU-MIMO system consists of a base station (BS) equipped with $N_{t}$ antennas that serves to $M$ mobile stations (MSs) or user equipments (UEs), as seen in Fig. 2.2. The $m$ th $\mathrm{MS}$ is equipped with $N_{m}$ antennas.

MU-MIMO differs from SU-MIMO in the fact that the different users share the spatial dimension, causing multiuser interference. This interference is managed through the use of multiple antennas, allowing the spatial separation of the different users. On the uplink or multiple access channel (MAC), the MU-MIMO techniques appeared as a generalization of SU-MIMO for the multiuser case. However, the downlink or broadcast channel (BC) presents more challenges since it is expected to be more rate-demanding in practical systems [6].

The advantages of MU-MIMO over SU-MIMO have motivated an extensive research on this field over the last few years. The main advantages are listed below $[6]$ : 


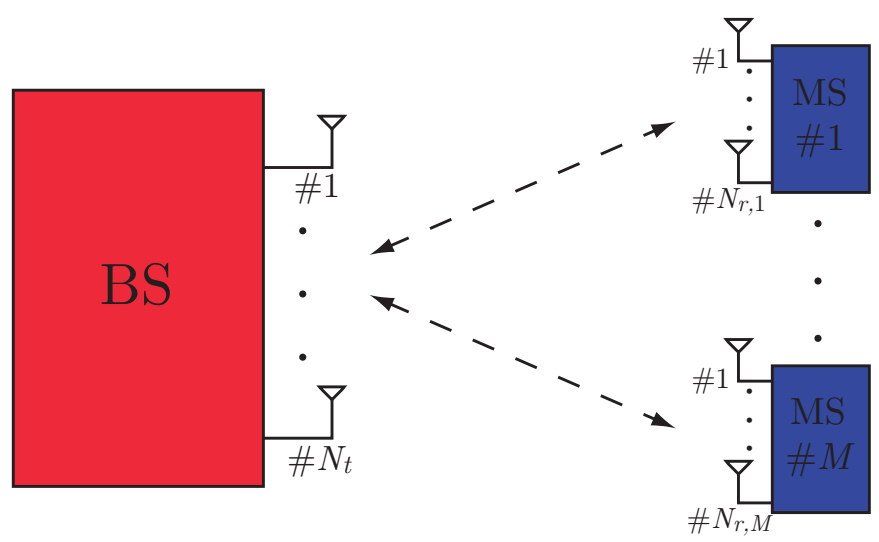

Figure 2.2: MU-MIMO system.

- A gain proportional to the number of antennas at the BS is obtained through multiuser multiplexing schemes.

- User scheduling techniques provide higher immunity against the main limitations in SU-MIMO schemes like ill-conditioned channels. Furthermore, unlike SU-MIMO, line-of-sight propagation does not cause degradation in spatial multiplexing schemes.

- MU-MIMO allows spatial multiplexing without requiring multiple antennas at the MSs, keeping most part of the hardware at the transmitter side and allowing the use of small and inexpensive terminals.

However, all these benefits come at the cost of a higher complexity in the system. Most of MU-MIMO techniques require CSIT, which usually has to be provided by the MSs. Thus, the efficiency of the uplink decreases, especially in wideband or high-mobility systems. In addition, tasks such as scheduling, precoding or feedback in these scenarios may suffer from a prohibitive computational complexity.

\section{Uplink}

In the uplink or MAC (see Fig. 2.3), the received signal at the BS can be expressed as

$$
\mathbf{y}=\sum_{m=1}^{M} \mathbf{H}_{m}^{H} \mathbf{x}_{m}+\mathbf{n},
$$

where $\mathbf{y} \in \mathbb{C}^{N_{t} \times 1}$ is the received signal at the BS, $\mathbf{H}_{m} \in \mathbb{C}^{N_{m} \times N_{t}}$ is the channel matrix for the $m$ th MS, $\mathbf{x}_{m} \in \mathbb{C}^{N_{m} \times 1}$ is the signal transmitted by the $m$ th MS and $\mathbf{n} \in \mathbb{C}^{N_{t} \times 1}$ represents i.i.d. circularly-symmetric complex Gaussian noise vector, 
with zero mean and unit variance, $\mathcal{C N}(0,1)$. The power constraint for the $m$ th MS is expressed as $\operatorname{Tr}\left(\mathbf{Q}_{m}\right) \leq P_{m}$, where $\mathbf{Q}_{m}$ is the covariance matrix of the transmitted signal, $\mathbf{Q}_{m}=\mathbb{E}\left[\mathbf{x}_{m} \mathbf{x}_{m}^{H}\right]$.

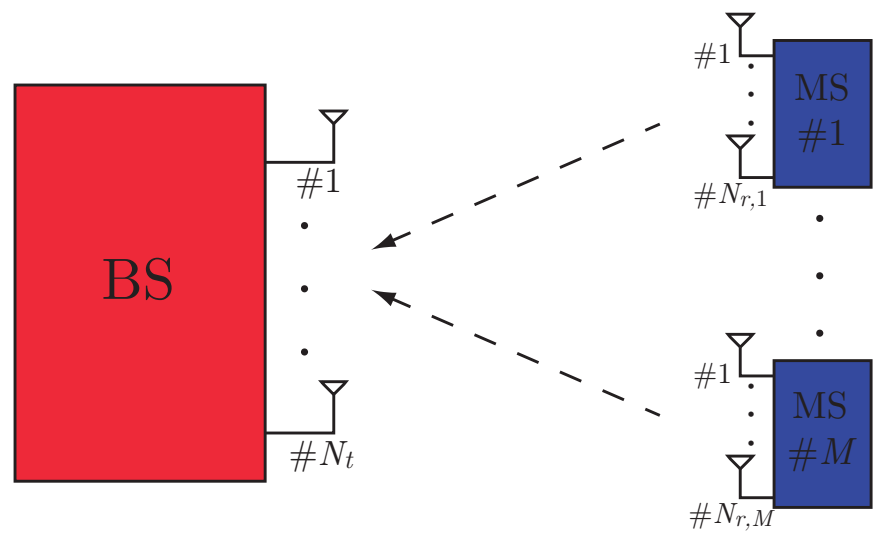

Figure 2.3: Uplink of a MU-MIMO system.

In MU-MIMO systems, the capacity is not given by a scalar value but by a $M$-dimensional region that contains the set of all rate vectors $\left(R_{1}, \ldots, R_{M}\right)$ simultaneously achievable by all $M$ MSs. Assuming a perfect knowledge of the different channel matrices, $\mathbf{H}_{m}$, and any set of power constraints $\mathcal{P}=\left(P_{1}, \ldots, P_{m}\right)$, the capacity region of the MIMO MAC has been obtained in $[26,37]$ and is given by

$$
\begin{aligned}
& C_{\mathrm{MAC}}\left(\mathcal{P}, \mathbf{H}^{H}\right)= \\
& =\underset{\substack{\mathbf{Q}_{m} \geq 0, \operatorname{Tr}\left(\mathbf{Q}_{m}\right) \leq P_{m}, \forall m}}{\bigcup}\left\{\begin{array}{l}
\left(R_{1}, \ldots, R_{M}\right): \\
\sum_{m=1}^{M} R_{m} \leq \log _{2} \operatorname{det}\left(\mathbf{I}+\sum_{m=1}^{M} \mathbf{H}_{m}^{H} \mathbf{Q}_{m} \mathbf{H}_{m}\right)
\end{array}\right\} .
\end{aligned}
$$

Considering the transmitted signals as zero-mean Gaussian random variables with spatial covariance matrix $\mathbf{Q}_{m}$, the set of covariances matrices $\left(\mathbf{Q}_{1}, \ldots, \mathbf{Q}_{m}\right)$ corresponds to a $M$-dimensional polyhedron of achievable rates

$$
\left\{\left(R_{1}, \ldots, R_{M}\right): \sum_{m=1}^{M} R_{m} \leq \log _{2} \operatorname{det}\left(\mathbf{I}+\sum_{m=1}^{M} \mathbf{H}_{m}^{H} \mathbf{Q}_{m} \mathbf{H}_{m}\right)\right\}
$$

and the capacity region is equal to the union over all covariance matrices satisfying the trace constraints of all such polyhedrons. The corner points of each polyhedron can be achieved by successive decoding, in which users' signals are successively decoded and subtracted out of the received signal. For the two-user case, each set of covariance matrices corresponds to a pentagon, as shown in Fig. 2.4 [26]. 


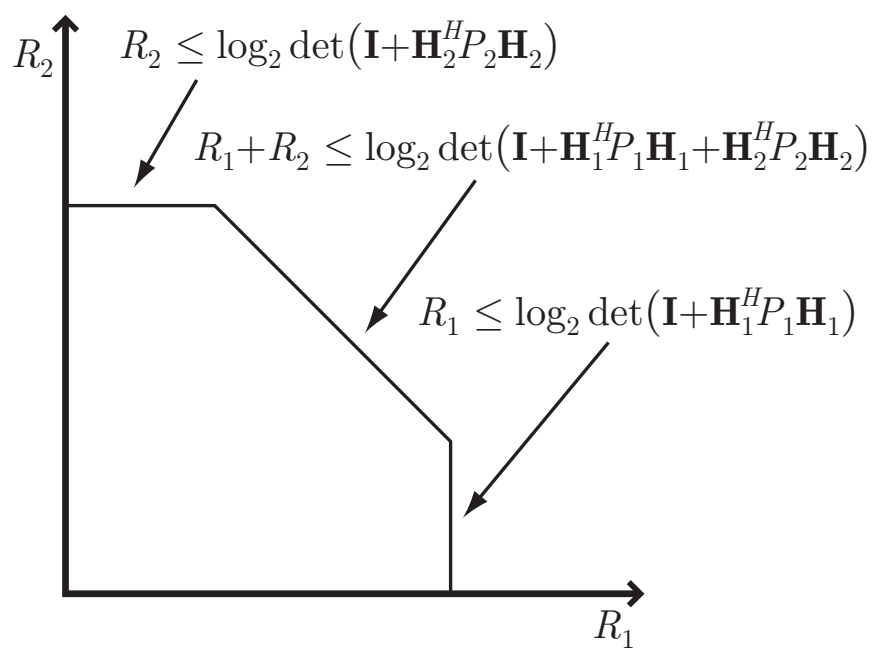

Figure 2.4: Capacity region of the MIMO MAC for $M=2$ and $N_{1}=N_{2}=1$.

\section{Downlink}

In the downlink or BC (see Fig. 2.5), the received signal at the $m$ th MS can be expressed as

$$
\mathbf{y}_{m}=\mathbf{H}_{m} \mathbf{x}+\mathbf{n}_{m},
$$

where $\mathbf{y}_{m} \in \mathbb{C}^{N_{m} \times 1}$ contains the received symbols, $\mathbf{H}_{m} \in \mathbb{C}^{N_{m} \times N_{t}}$ is the downlink channel matrix and $\mathbf{n}_{m}$ is the i.i.d. circularly-symmetric complex Gaussian noise, $\mathcal{C N}(0,1)$, at the $m$ th MS. Vector $\mathbf{x} \in \mathbb{C}^{N_{t} \times 1}$ represents the transmitted signal, which contains information for all the MSs, and its covariance matrix is given by $\mathbf{Q}=\mathbb{E}\left[\mathbf{x x}^{H}\right]$. An example of transmitted signal is $\mathbf{x}=\sum_{m=1}^{M} \mathbf{x}_{m}$, where $\mathbf{x}_{m}$ contains the information for the $m$ th MS. The power allocated to the $m$ th MS is determined by $\operatorname{Tr}\left(\mathbf{Q}_{m}\right)$, where $\mathbf{Q}_{m}$ is the spatial covariance matrix given by $\mathbf{Q}_{m}=\mathbb{E}\left[\mathbf{x}_{m} \mathbf{x}_{m}^{H}\right]$, and the sum-power constraint is expressed as $\sum_{m=1}^{M} \operatorname{Tr}\left(\mathbf{Q}_{m}\right)=$ $\operatorname{Tr}(\mathbf{Q}) \leq P[26]$. Per antenna power constraints have been considered in [38].

A general review of MU-MIMO systems has been presented in [39]. From an information theoretic point of view, the multiuser interference can be pre-cancelled with no extra power cost in transmission using the concept of DPC. If the interference is perfectly known at the transmitter, DPC states that the channel capacity is the same as if the interference was not present [40]. It has been proven that $\mathrm{DPC}$ is optimal in the sense that it achieves the sum-capacity of the Gaussian 


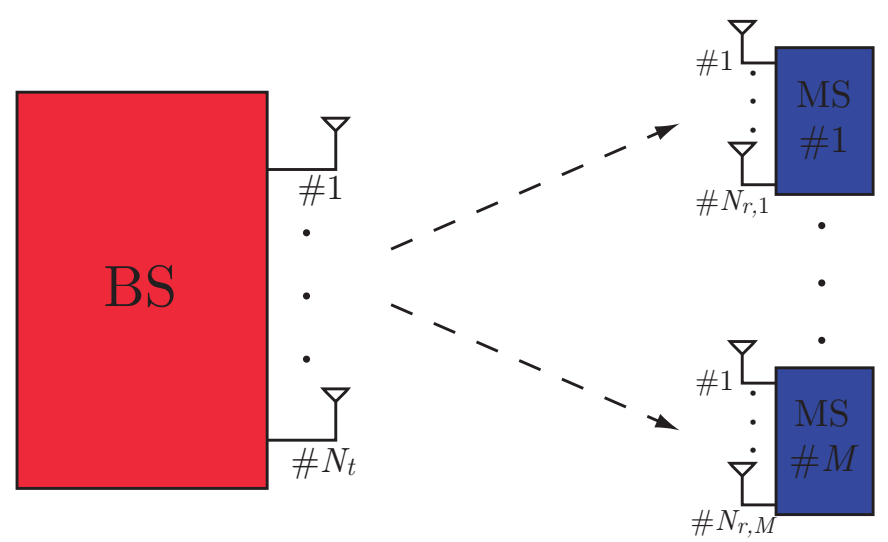

Figure 2.5: Downlink of a MU-MIMO system.

MIMO BC [41-44]. The components of the achievable rate vector are given by

$$
R_{\pi(m)}=\log _{2} \frac{\operatorname{det}\left(\mathbf{I}+\mathbf{H}_{\pi(m)}\left(\sum_{j \geq m} \mathbf{Q}_{\pi(j)}\right) \mathbf{H}_{\pi(m)}^{H}\right)}{\operatorname{det}\left(\mathbf{I}+\mathbf{H}_{\pi(m)}\left(\sum_{j>m} \mathbf{Q}_{\pi(j)}\right) \mathbf{H}_{\pi(m)}^{H}\right)},
$$

where $\pi(m)$ represents the encoding ordering of the $m$ th MS. The dirty paper region is defined as the convex hull of the union of all the rate vectors over all the covariance matrices that fulfill the power constraint $\operatorname{Tr}\left(\mathbf{Q}_{1}+\ldots+\mathbf{Q}_{M}\right) \leq P$ and over all permutations $(\pi(1), \ldots, \pi(M))[26,27]$ :

$$
C_{\mathrm{DPC}}(P, \mathbf{H})=\bigcup_{\pi, \mathbf{Q}_{m}} \mathbf{r}\left(\pi, \mathbf{Q}_{m}\right),
$$

where $\mathbf{r}\left(\pi, \mathbf{Q}_{m}\right)$ is the rate vector obtained from (2.11). Figure 2.6 shows the DPC region for a system where a BS equipped with $N_{t}=2$ antennas serves to $M=2$ single-antenna MSs [26].

The dirty paper region may be difficult to compute since functions in (2.11) are not either concave or convex and an exhaustive search over all the covariance matrices that meet the power constraint is required. In $[42,45,46]$, the capacity region is obtained making use of the duality relationship between MIMO MAC and MIMO $\mathrm{BC}$. Through this duality, the dirty paper region of the $\mathrm{BC}$ with power constraint $P$ is obtained from the union of regions of the dual MAC that meet the sum power constraint $P$. Although the DPC technique requires an extremely high complexity, it has given rise to numerous suboptimal techniques involving linear and nonlinear precoding $[15,20-22,47]$. 


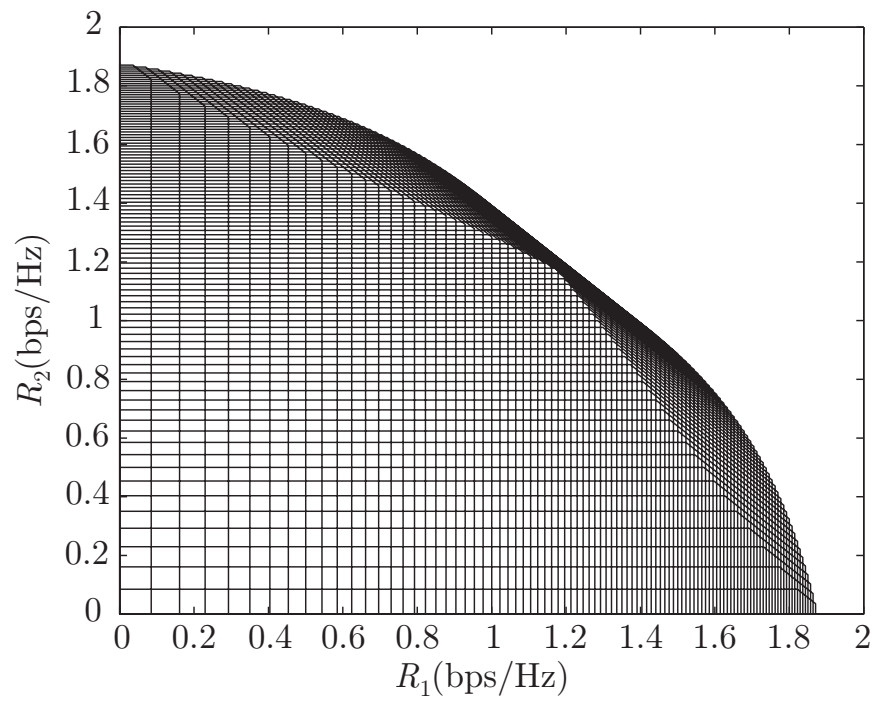

Figure 2.6: Dirty paper region for $N_{t}=2, M=2, N_{1}=N_{2}=1, \mathbf{H}_{1}=\left[\begin{array}{ll}1 & 0.5\end{array}\right]$, $\mathbf{H}_{2}=\left[\begin{array}{ll}0.5 & 1\end{array}\right]$ and $P=10[26]$.

\subsection{Coordinated systems}

Interference is one of the main limitations of traditional wireless communication systems [48]. In mobile communications systems, the intercell interference (ICI) forces to reduce the reuse of the channels (time, frequency or codes) along the network, which entails a reduction in the overall spectral efficiency expressed in $\mathrm{bps} / \mathrm{Hz} / \mathrm{BS}$ [49]. Alternatively, a full reuse can be kept at the cost of a high interference at the cell edge. In this area, the data rate strongly decays as a consequence of the ICI, causing an inhomogeneous distribution of the data rate along the network. The primary results about the effect of the interference in wireless communication systems are collected in [50-54].

Network coordination emerges as a technique capable of reducing the ICI and increasing the data rate at the cell edge, thus improving the spectral efficiency of the system. This technique is also known as cooperative MIMO [55] and network MIMO [56] in the literature. The main idea of network coordination resides in the use of the backhaul links that interconnect the different BSs in order to allow a coordinated transmission and/or reception.

As a first approach, a system where a set $\mathcal{B}$ of BSs (equipped with $N_{t}$ antennas each one) are connected to a central unit through backhaul links of infinite capacity, was considered [57-59]. Ignoring the individual power constraints of the BSs or antennas, this system can be viewed as a large MU-MIMO with a 'super' BS 
with distributed antennas. Assuming a synchronous reception from all the BSs and neglecting the interference from outside the coordinated cluster, the received signal at the $m$ th MS can be expressed as (see Fig. 2.7):

$$
\mathbf{y}_{m}=\sum_{b=1}^{|\mathcal{B}|} \mathbf{H}_{m, b} \mathbf{x}_{b}+\mathbf{n}_{m}
$$

where $\mathbf{H}_{m, b} \in \mathbb{C}^{N_{m} \times N_{t}}$ is the downlink channel matrix between the $b$ th BS and the $m$ th MS, vector $\mathbf{x}_{b} \in \mathbb{C}^{N_{t} \times 1}$ represents the transmitted signal by the $b$ th BS and $\mathbf{n}_{m}$ is noise vector at the $m$ th MS. Aggregating the channel matrices and the transmitted signals of the different BSs, (2.13) can be expressed as

$$
\mathbf{y}_{m}=\mathbf{H}_{m} \mathbf{x}+\mathbf{n}_{m},
$$

where $\mathbf{H}_{m}=\left[\mathbf{H}_{m, 1}, \ldots, \mathbf{H}_{m,|\mathcal{B}|}\right] \in \mathbb{C}^{N_{m} \times|\mathcal{B}| N_{t}}$ is the aggregated channel matrix and $\mathbf{x}=\left[\mathbf{x}_{1}^{H}, \ldots, \mathbf{x}_{|\mathcal{B}|}^{H}\right]^{H} \in \mathbb{C}^{|\mathcal{B}| N_{t} \times 1}$ is the aggregated transmitted signal. Thus, up to $|\mathcal{B}| N_{t}$ MSs can be spatially multiplexed using MU-MIMO techniques.

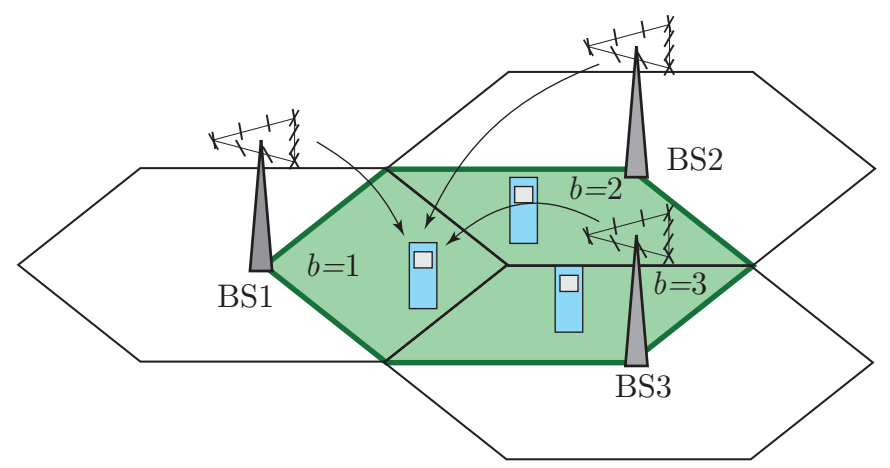

Figure 2.7: Example of a coordinated cluster of $|\mathcal{B}|=3$ cooperating sectors and $N_{t}=4$ transmit antennas per sector.

However, the practical implementation of this system entails more realistic assumptions such as per-BS or per-antenna power constraints [60, 61], finite capacity of the backhaul [62-64], some latency in the communications between the BSs [64] and asynchronous reception at the MSs [65]. In addition, the computational complexity of some algorithms increases rapidly as the dimensions of the system become larger [49].

Within the standard LTE-Advanced, the network coordination techniques are known as coordinated multipoint (CoMP) transmission and reception [66]. A general description of this technique can be found in [11, 49, 67-69]. The performance evaluation of CoMP in practical implementations has been presented in $[70,71]$. 


\subsection{Massive MIMO systems}

Massive MIMO systems, a.k.a. very large MIMO systems, appear to avoid the limitations related to the backhaul communication due to the coordination of different BSs. Massive MIMO systems consist of a very large array of antennas serving a smaller number of MSs. Whereas LTE-Advanced allows for BSs with up to 8 antennas, massive MIMO entails the use of hundreds of antennas. In this case, the number of MSs is not limited by the number of antennas but by the difficulty of obtaining CSIT from a large number of MSs [72]. Different antenna configurations and deployments are shown in Fig. 2.8.

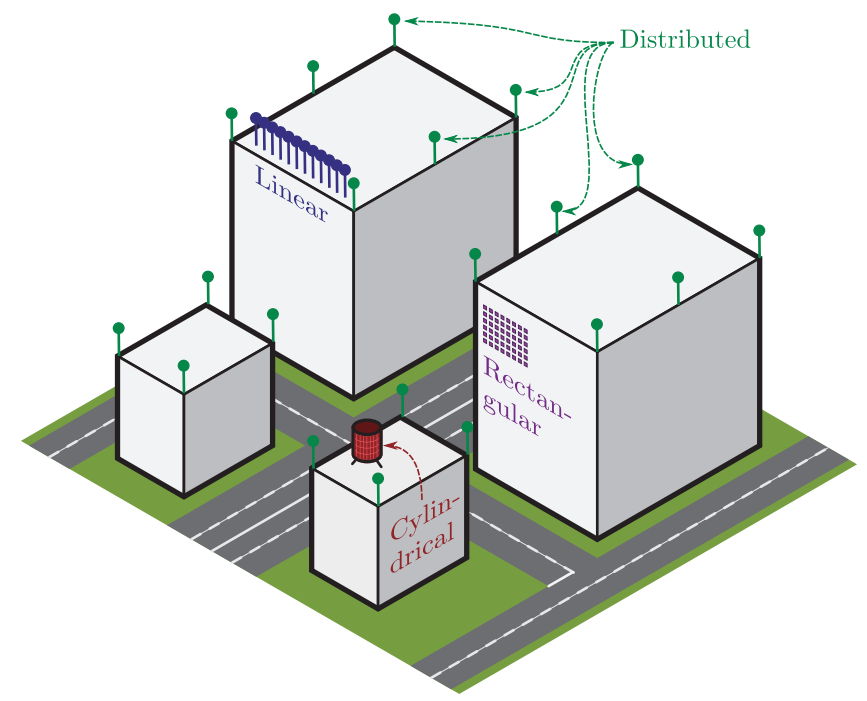

Figure 2.8: Some possible antenna configurations and deployment scenarios for a massive MIMO base station [73].

The main advantage of massive MIMO in cellular systems is the ability to considerably reduce the ICI with low-complexity techniques [74]. In addition, massive MIMO enhances even more the advantages of MIMO [72, 73]:

- Massive MIMO can increase the capacity around 10 times through spatial multiplexing, improving the radiated energy-efficiency in the order of 100 times.

- Keeping the total transmitted power constant and increasing the number of antennas results in a lower power per antenna. Thus, low-power components can be used in massive MIMO systems, resulting in less expensive equipment. 
- Due to its additional degrees of freedom, massive MIMO systems have a higher robustness against interference. Additionally, they are also more robust against the failure of the antenna units.

With very large antenna arrays, some random properties become deterministic, such as the distribution of the singular values of the channel matrix. In addition, the channel vectors for the different users become more orthogonal as the number of antennas increases [72]. These effects allow the simplification of the MAC techniques and reduce the effects of fading dips [73].

One of the main problems of these systems lies in obtaining CSIT. First, the channel estimation process suffers from pilot contamination due to the reuse of non-orthogonal pilot sequences in contiguous cells [74]. This problem has been analyzed and some solutions are proposed in [75-79]. Second, the amount of CSI sent by the MSs in the uplink is larger since it is proportional to the number of antennas at the BS. Generally, this problem is solved using TDD systems, which allow the use of the reciprocal properties of the channel [74]. Other problem which complicates the practical implementation of this system involves the physical installation of large arrays in space-limited BSs [80, 81].

The capacity and achievable rates of these systems under different assumptions has been analyzed in [74, 79, 82-88]. Results presented in [89, 90] involve some cooperation between BSs, but they can be useful since they present the performance for the large system limit. Some downlink techniques such as resource allocation, modulation and precoding are studied in [91-93]. Finally, [80] covers the practical implementation of these systems, showing system-level simulations, and [94] presents a prototype with $N_{t}=64$ antennas serving to $M=15 \mathrm{MSs}$

\subsection{MIMO-OFDM}

OFDM has become a very attractive technique in wireless communications during the last decades. OFDM was first proposed in [95, 96], but its high implementation complexity limited its use. However, since an efficient implementation using the discrete Fourier transform (DFT) was proposed in [97], it has been used in many systems and adopted in different standards, such as IEEE 802.11a, IEEE 802.11g, IEEE 802.11n, 802.16 WiMAX (see [98, Chap. 2], [8] and references therein) and LTE/LTE-Advanced (see [11, Chap. 5], [99, Chap. 3]).

OFDM is used to mitigate the effects of ISI in frequency selective channels, turning a broadband frequency selective channel into a set of parallel narrowband frequency flat subchannels. A high spectral efficiency is achieved since the frequency separation between subcarriers is the minimum required to preserve the orthogonality. In addition, different signal constellations and different power loadings can 
be used along the different subcarriers in order to optimize the throughput or to assure a certain quality of service (QoS).

In OFDM systems, $K$ information symbols are transmitted in parallel on $K$ OFDM subcarriers. Thus, the time duration of an OFDM symbol is $K$ times larger than the symbol in a single-carrier system. The OFDM modulation can be efficiently implemented through the inverse discrete Fourier transform (IDFT) and a digitalto-analog converter. The ISI caused by the channel spread is eliminated by the cyclic prefix $(\mathrm{CP})$, which acts as a guard interval and whose length must be at least equal to the length of the multipath channel. In addition, it allows using the circular convolution as the linear convolution between the channel and the transmitted signal, allowing the use of efficient algorithms such as the fast Fourier transform (FFT) [7].

MIMO techniques can enhance the performance of OFDM by leveraging the spatial domain. OFDM simplifies the signal processing in MIMO techniques, since they are performed over narrowband flat-fading channels and each channel can be processed independently [100]. A general description of these systems can be found in [7, 8, 101], [102, Chap. 9], and some field results are presented in [100].

For each pair of transmit and receive antennas, the time-domain channel impulse response (CIR) is given by

$$
\mathbf{h}_{\mathrm{CIR}}=\left[h_{\mathrm{CIR}}[0], \ldots, h_{\mathrm{CIR}}[L-1]\right]^{T} \text {, }
$$

where $L$ denotes the number of samples in the CIR. The channel frequency response (CFR) for $K \geq L$ OFDM subcarriers is obtained as

$$
\mathbf{h}_{\mathrm{CFR}}=\sqrt{K} \mathbf{F}_{L} \mathbf{h}_{\mathrm{CIR}},
$$

where $\mathbf{h}_{\mathrm{CFR}}=\left[h_{\mathrm{CFR}}[0], \ldots, h_{\mathrm{CFR}}[K-1]\right]^{T}$ and $\mathbf{F}_{L}$ is an $K \times L$ matrix obtained by taking the first $L$ columns of a unitary $K \times K$ DFT matrix, whose elements are given by $\left[\mathbf{F}_{L}\right]_{k, l}=(1 / \sqrt{K}) e^{-j 2 \pi k l / K}$.

Assuming a cyclic prefix whose length is longer than the maximum delay of any channel path, the received signal on the $k$ th subcarrier in a MIMO system can be expressed similarly to 2.1 as

$$
\mathbf{y}[k]=\mathbf{H}[k] \mathbf{x}[k]+\mathbf{n}[k],
$$

where $\mathbf{H}[k]$ represents the CFR matrix, vector $\mathbf{x}[k]$ includes the transmitted symbols and $\mathbf{n}[k]$ is the noise vector on the $k$ th subcarrier [5]. Thus, (2.17) can be seen as $K$ multiple narrowband MIMO systems in parallel. 


\subsection{Limited Feedback}

As seen in sections 2.1-2.3, the performance of MIMO communications systems can be extremely strengthened if CSI is available at the transmitter. Channel adaptive signaling allows the continuous adaptation of the transmitted signal to the propagation conditions of the channels.

In FDD systems, a reasonably accurate CSIR can be easily obtained through techniques such as training. However, CSIT cannot be obtained directly from the reverse link since the uplink and downlink channels are separated in frequency and are usually uncorrelated. In these systems, CSIT must be provided by the receivers preferably through a low-rate feedback channel, commonly referred to as limited feedback schemes. The amount of feedback information depends on the system scenario and, generally speaking, is larger when the channel introduces some form of disturbance, such as spatial or multiuser interference [13]. Thus, designing limited feedback schemes to reduce the amount of feedback information plays an important role in the design of multiuser communication systems.

Limited feedback schemes for SU-MIMO and MU-MIMO systems have been extensively studied in the literature (see [13] and references therein, and the special issues $[103,104])$. This section presents a review of some of the most relevant limited feedback schemes for MIMO systems proposed in the existing literature.

\subsubsection{Single-user}

Although CSIT is not required to achieve the diversity gain (e.g., space-time codes), it is necessary in SU-MIMO techniques such as beamforming or interference mitigation at the transmitter side.

\section{Covariance adaptation}

From an information-theoretic point of view, the covariance adaptation technique allows the characterization of the performance of the system in terms of the maximum achievable rate [24]. In [105], the receiver provides some unquantized statistical CSI and the optimal covariance matrix of the transmitted signal is obtained from this information at the transmitter. A general review of feedback schemes based on statistical information has been carried out in [106].

In quantization-based feedback systems, both the transmitter and the receiver are assumed to have an identical codebook of possible covariance matrices. Making use of the CSIR, the receiver can select the optimal covariance matrix from the codebook and send its binary index to the transmitter. Lloyd's algorithm is used in [107] to efficiently design the codebook while random codebooks are analyzed 
in [108] showing that the capacity loss of MIMO systems decreases exponentially as a function of the number of feedback bits. If temporal correlation between channel realizations is considered, a better performance can be obtained through feedback schemes based on gradient methods for channel tracking [109] or codebooks which are dynamically adapted to leverage the correlation [110].

\section{Beamforming}

The importance of accurate CSIT in beamforming techniques can be extracted from the analysis in $[111,112]$. The simplest feedback scheme in beamforming systems is antenna selection, where the receiver sends back the index of the transmit antenna which maximizes the SNR and the data is transmitted only from this antenna [113], [114, Chap. 9]. The work in [115] assumes a similar envelope of the channel fading from all the antennas and only quantizes their phases using a uniform quantization. A more complex feedback scheme based on VQ is considered in [116], where the space of the channel vectors is divided into regions and the index of the region with a lower distortion is selected and sent to the transmitter. The performance of beamforming is analyzed as a function of the level of CSIT. A review of other different VQ approaches is presented in [117].

An alternative approach based on beamforming quantization instead of channel quantization has also been used in limited feedback systems. Since the receiver has a more accurate CSI than the transmitter, the receiver selects the beamforming vector from a codebook and send its index back to the transmitter. This approach has been widely employed in works such as $[113,118,119]$. The problem of the optimal codebook design for spatially uncorrelated Rayleigh fading channel has been addressed in [120], showing that it is equivalent to the Grassmannian line packing problem. Basically, this problem consists of maximizing the sine of the minimum angular separation between any two vectors of the codebook. A systematic codebook construction based on rotations and scalings of the Grassmannian line packing for correlated channels has been proposed in [121].

The Grassmannian line packing problem is still far from trivial and requires a high computational complexity. An alternative approach to generate the beamforming codebooks is using VQ techniques. A Lloyd-like algorithm that iteratively constructs the codebook is proposed in [122]. A rate-distortion analysis and high resolution analysis of VQ have been carried out in [123] and [124], respectively. Codebook designs for correlated Rayleigh fading channels have been performed making use of the rate-distortion analysis [123] and the high resolution analysis [125]. A much more simple technique such as random generation of the codebooks has been proposed in [126] and thoroughly analyzed in [127]. 


\section{Spatial multiplexing}

Similarly to the beamforming case, the first limited feedback schemes focused on channel quantization [128]. The performance of the subsequent feedback schemes was improved by allowing the receiver to directly design the precoder. The simplest feedback schemes for precoding is the antenna subset selection technique [129, 130]. In these systems, the receiver selects the optimum subset among all possible transmit and receive antennas according to some performance (maximum rate, minimum error, etc.). Codebooks for linear precoding based on Grassmannian line packing [131] and random vector quantization (RVQ) $[132,133]$ have also been proposed.

\section{Broadband systems}

Latest wireless communications standards, such as LTE/LTE-A or WiMAX, have adopted the MIMO-OFDM technology in the physical layer. As seen in section 2.4, characterizing the full CSI may require much information for a large number of OFDM subcarriers or a long CIR. Since the signal processing is performed on a per-subcarrier basis, this information is required at the transmitter and receiver. CSIR at the pilot subcarriers can be obtained through training, but it can be difficult to obtain in the rest of subcarriers. The work in $[134,135]$ proposes to quantize the beamforming vectors and the precoding matrices of a set of subcarriers (i.e., pilot subcarriers) and send them back to the transmitter. Taking advantage of the frequency correlation, the beamforming vectors at the rest of subcarriers are obtained by spherical interpolation. Other interpolation methods based on the Grassmannian line packing problem have been proposed, such as the weighted least squares approach presented in [136] or the geodesic interpolation in [137].

An alternative approach with a lower complexity than interpolation is clustering. This technique consists of dividing all the subcarriers into different sets or clusters, and the subcarriers within the same set use the same beamforming vector or precoding matrix $[134,135,138]$.

\subsubsection{Multiuser}

Multiuser communication systems introduce an additional degree of freedom and can provide a large improvement in the system rate when the spatial resources are spread among multiple users [13]. However, the amount of CSIT required increases with the number of users and it is essential for multiuser interference mitigation. Since this thesis is focused on MU-MIMO systems with single-antenna MSs, the review of literature pays special attention to single-antenna receivers.

The main difference between feedback schemes for single-user systems and multiuser systems is the amount of CSI at the receiver side. While in single-user 
systems the receiver can easily estimate the full channel matrix, MSs usually cannot cooperate in multiuser systems and do not have any information about the rest of MSs. Thus, each MS is unable to obtain the optimal precoder or beamformer [139].

\section{Narrowband systems}

Limited feedback schemes for multiuser FDD systems can be mostly classified into two categories. In the first category, the MSs quantize the channel vector/matrix (or a function of it) and send the quantized information to the BS. One of the first schemes was proposed in [140], where the feedback information consists of pilot symbols or reference signals (RSs) received at the MSs. Due to the quantization error, multiuser interference cannot be fully cancelled and the system becomes interference limited, leading to a system rate ceiling for high SNR [141-143]. The advantages of quantized (digital) feedback over unquantized (analog) feedback in terms of achievable rates have been analyzed in [144]. In the analysis, the effects of imperfect channel estimation and delayed feedback have been considered.

Regarding the quantized information that is fed back, two different feedback approaches are compared in [145]. The first approach is based on channel quantization and the second is based on linear precoder quantization. In the scenario under evaluation, the approach based on channel quantization has shown a higher sum-rate. Feedback based on channel quantization is also used in [146] to determine the number of scheduled MSs that maximizes the throughput per antenna. Another advantage of this approach is that it does not restrict the precoding technique, allowing the use of non-linear precoding [139, 147]. However, the approach based on precoder quantization often leads to a higher performance when linear precoding is used $[148,149]$.

The limited feedback schemes in the second category are based on opportunistic beamforming [13]. The work in [150] uses multiple spatial beams chosen randomly according to a known distribution. The different MSs provide the received signalto-interference-plus-noise ratio (SINR) through the feedback link and the MSs with the highest SINRs are scheduled. The asymptotic analysis shows that the system throughput achieves the same scaling law of the sum-rate capacity when perfect CSI is available, as also shown in [151]. The special case of 1-bit of quantized SINR is analyzed in [152], showing that multiuser diversity is achievable even with a minimum feedback overhead. Using ranking-based CSIT can improve the temporal fairness between MSs, as shown in [153]. Interesting trade-offs between the number of feedback bits, the number of MSs and the SINR are presented in [154].

Although, opportunistic beamforming techniques show a good performance when the number of MSs in the system is large, its performance quickly degrades for 
practical values of MSs. This problem is addressed in [155]. Authors in this work propose a framework where the problems of scheduling and beamforming are decoupled. The main idea is that, once the scheduling has been performed, additional CSIT can be requested in order to improve the performance of the beamforming (compared to random beamforming). Per user unitary rate control (PU2RC) is another technique that appears as a generalization of opportunistic beamforming, where the codebook is constructed from multiple orthonormal basis. The performance of PU2RC for an asymptotically large number of MSs is analyzed in $[156]$.

\section{Broadband systems}

Limited techniques for multiuser broadband systems appear as a generalization of the single-user case, where subcarrier clustering (the total number of subcarriers are divided into sets) is often used in order to leverage frequency correlation [134]. A feedback scheme based on clustering and only providing the information about the strongest clusters is proposed in [157]. Similarly, [158] proposes three different feedback schemes for opportunistic beamforming in MIMO-OFDMA systems. In these schemes, each user only feeds back the indices of those subcarriers where their rate is maximized according to different expressions. The scheme in [159] incorporates antenna combining and subcarrier clustering to further reduce the quantization distortion.

The scheme proposed in [160] is also based on subcarrier clustering, where the spectral width of the clusters is chosen according to the coherence bandwidth. In addition, the bit allocation regarding the quantization of the different clusters is analyzed. A comparison between analog feedback, direction quantized feedback [141] and time-domain channel quantized feedback is carried out in [161]. Similarly to the single-user case, subcarrier clustering has been used in the first two schemes in order to exploit frequency correlation [134]. Results show that time domain quantization is simpler to implement and achieves the best performance.

\section{Coordinated systems}

In coordinated systems, MSs have to estimate and provide the CSI related to multiple BSs. In addition, BSs need to share CSI and MS data via backhaul links of finite capacity. Thus, limited feedback schemes are also crucial in order to decrease the high amount of CSI necessary in coordinated systems [49, 162].

Different degrees of cooperation have been proposed in the literature, offering a trade-off between performance and the amount of overhead on the backhaul and on the feedback link. In order to limit the base station cooperation, dynamic clustering is proposed in $[163,164]$. Only a subset of MSs is selected to work with 
coordinated techniques in [163], whereas a greedy technique for the clustering formation is used in [164].

Static clustering is considered in [165], where it is shown that most of the sum rate benefits from coordinated systems can be achieved with small-sized clusters (about 7 cells), leading to an important decrease in the signaling overhead compared to the global coordination case. The use of overlapping clusters in [166] performs very close to the full coordinated system. Practical aspects of the trade-off between achievable rate and backhaul usage are discussed in [167].

Additional research is mainly focused on reducing the feedback overhead, which also brings a reduction to the amount of information on the backhaul. A subspacebased channel quantization method and a hierarchical codebook design that leverages the temporal correlation are proposed in [168]. A limited feedback technique based on selective feedback is proposed in [169], where the MSs only have to provide those channel coefficients that are above a threshold. In addition, scheduling and precoding schemes are also proposed in order to reduce the backhaul overhead.

The development of signal processing techniques involving local CSI and MS data has also become important in coordinated systems. The objective is to achieve a performance close to the ideal cooperative case with a reduced overhead mainly on the backhaul, but also on the feedback link [49]. Distributed precoding techniques where each BS only has local CSI have been analyzed in $[63,170]$

\subsection{Channel models}

The statistical properties of the propagation channel determines the performance of MIMO systems. In fact, most of the techniques used in these systems are strongly related to the propagation conditions. Thus, characterizing the MIMO channel is of key importance in the design and simulation of MIMO communication systems. Detailed overviews of the existing channel models have been presented in $[171,172]$. This section collects the channel models that are used throughout this thesis. As in the previous section, MU-MIMO systems with single-antenna MSs are considered. 


\subsubsection{Gaussian i.i.d. model}

From a theoretical point of view, it can be useful to consider the elements of the narrowband MIMO channel matrix as i.i.d. circularly-symmetric complex Gaussian random variables, with zero mean and unit variance, $\mathcal{C N}(0,1)$ (e.g., [23]). This model corresponds to a rich scattering propagation environment and a large spacing between elements in the transmit antenna array. Although the analysis of this model can be useful (see [173]), it does not reflect the effects of limited scattering environments and small antenna separations present in practical systems.

\subsubsection{Kronecker model}

The Kronecker model introduces the effect of the correlation in the channel model. This model assumes that the spatial covariance matrix can be written as the Kronecker product of the transmit and receive covariance [174, 175]. Focusing on a multiuser system with single-antenna MSs, the channel vector for the $m$ th MS is expressed as

$$
\mathbf{h}_{m}=\mathbf{C}_{m}^{1 / 2} \mathbf{g}_{m},
$$

where $\mathbf{C}_{m}=\mathbb{E}\left[\mathbf{h}_{m} \mathbf{h}_{m}^{H}\right] \in \mathbb{C}^{N_{t} \times N_{t}}$ is the spatial covariance $^{1}$ matrix at the transmitter experienced by the $m$ th MS and $\mathbf{g}_{m} \in \mathbb{C}^{N_{t} \times 1}$ is a vector whose elements are i.i.d. circularly-symmetric complex Gaussian variables, with zero mean and unit variance, $\mathcal{C N}(0,1)$. The matrix $\mathbf{C}_{m}^{1 / 2}$ denotes any matrix that meets $\mathbf{C}_{m}^{1 / 2}\left(\mathbf{C}_{m}^{1 / 2}\right)^{H}=\mathbf{C}_{m}$. Previous research [177, 178] has shown that the Kronecker model results in poor estimates for capacity.

\subsubsection{Extended ITU model}

In order to evaluate the $20 \mathrm{MHz}$ LTE channel, the International Telecommunication Union (ITU) extended the pedestrian and urban models presented in $[179,180]$. The main parameter that describes these channel models is the power delay profile of the multipath fading. The Extended Pedestrian A (EPA) and the Extended Typical Urban (ETU) have a root mean square (RMS) delay spread of 43 and $991 \mathrm{~ns}$, respectively. The power delay profile of these models are presented in [181] and collected in Table 2.1.

\footnotetext{
${ }^{1}$ In this case, the covariance matrix matches the correlation matrix because the elements of $\mathbf{h}_{m}$ have zero mean and unit variance [176]. Thus, this matrix will be referred to by using both terms.
} 
Table 2.1: Power delay profiles of extended ITU models.

\begin{tabular}{|c|c|c|c|c|}
\cline { 2 - 5 } \multicolumn{1}{c|}{} & \multicolumn{2}{c|}{ EPA model } & \multicolumn{2}{c|}{ ETU model } \\
\hline Tap number & $\begin{array}{l}\text { Relative } \\
\text { delay (ns) }\end{array}$ & $\begin{array}{l}\text { Relative mean } \\
\text { power }(\mathrm{dB})\end{array}$ & $\begin{array}{l}\text { Relative } \\
\text { delay (ns) }\end{array}$ & $\begin{array}{l}\text { Relative mean } \\
\text { power (dB) }\end{array}$ \\
\hline 1 & 0 & 0.0 & 0 & -1.0 \\
2 & 30 & -1.0 & 50 & -1.0 \\
3 & 70 & -2.0 & 120 & -1.0 \\
4 & 80 & -3.0 & 200 & 0.0 \\
5 & 110 & -8.0 & 230 & 0.0 \\
6 & 190 & -17.2 & 500 & 0.0 \\
7 & 410 & -20.8 & 1600 & -3.0 \\
8 & & & 2300 & -5.0 \\
9 & & & 5000 & -7.0 \\
\hline
\end{tabular}

\subsubsection{Spatial channel model}

The SCM was developed by the 3GPP and 3GPP2 in order to evaluate different MIMO techniques for high-speed downlink packet access (HDSPA) in a more realistic channel [182]. The SCM is a parametric stochastic channel model that characterizes the MIMO channels through geometrical parameters such as angle of arrival (AoA) and angle of departure (AoD). The received signal is modeled as a superposition of waves that describe the changes in the CIR between each pair of antennas.

In the SCM, system simulations consist of multiple BSs deployed in a multicell network with multiple MSs. Three different propagation environments are available: suburban macrocell, urban macrocell and urban microcell. The number of scatterer cluster determine the number of resolvable paths, which is fixed to $S=6$. The delay spread (DS) and the angular spread (AS) of the different paths are random variables. The number of subpaths within a path is $R=20$, and their angular offsets are also fixed and depend on the different propagation environments. Figure 2.9 shows the angular parameters of the SCM for the case of single-antenna MSs and Table 2.2 shows the parameters used in each of the environments. 


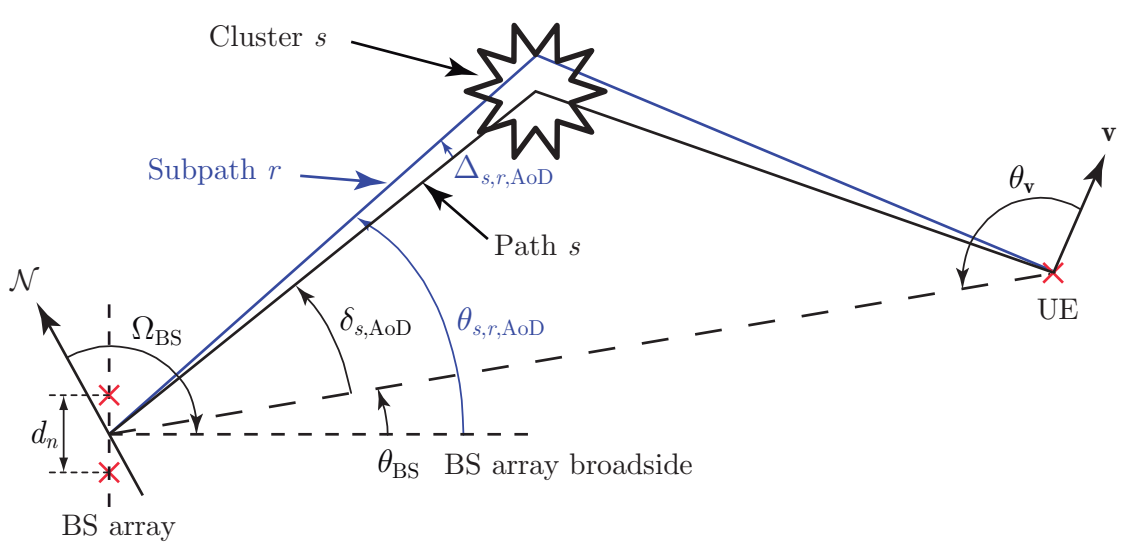

Figure 2.9: Angular parameters in SCM specifications (see [182] for more details)

Following [182], the coefficients of the $S$-multipath channel between each singleantenna MS and the $n$th antenna of the sector array are given by:

$$
h_{n s}=\sqrt{\frac{P_{s} \sigma_{\mathrm{PL}} \sigma_{\mathrm{SF}}}{R}} \sum_{r=1}^{R}\left(\begin{array}{l}
\left.\sqrt{G_{\mathrm{BS}}\left(\theta_{s, r, \mathrm{AoD}}\right)} \exp \left(j\left[k d_{n} \sin \left(\theta_{s, r, \mathrm{AoD}}\right)+\Phi_{s, r}\right]\right) \times\right), \\
\exp \left(j k\|\mathbf{v}\| \cos \left(\theta_{\mathbf{v}}\right) t\right)
\end{array}\right),
$$

where

$\begin{array}{ll}P_{s} & \text { is the normalized power of the } s \text { th path (the total power } \\ & \left.\text { for the } S \text { paths is equal to one, } \sum_{s=1}^{S} P_{s}=1\right) . \\ & \text { is the path loss. As seen in Table } 2.2 \text {, the model of path } \\ & \text { loss depends on the environment. } \\ & \text { is the lognormal shadow fading. } \\ \sigma_{\mathrm{SF}} & \text { is the number of subpaths per path. } \\ \theta_{s, r, \mathrm{AoD}} & \text { is the AoD for the } r \text { th subpath of the } s \text { th path. } \\ G_{\mathrm{BS}}\left(\theta_{s, r, \mathrm{AoD}}\right) & \text { is the BS antenna gain of each array element. } \\ k & \text { is the wave number } 2 \pi / \lambda, \text { where } \lambda \text { is the carrier wavelength. } \\ d_{n} & \text { is the distance from the } n \text {th antenna element to the refer- } \\ \Phi_{s, r} & \text { ence antenna element }(n=1) \text { at the BS. } \\ \|\mathbf{v}\| & \text { is the phase of the } r \text { th subpath of the } s \text { th path. } \\ \theta_{\mathbf{v}} & \text { is the magnitude of the MS velocity vector. } \\ & \text { is the angle of the MS velocity vector. }\end{array}$

One of the characteristics of the SCM channel is that the channel is generated without explicitly setting any spatial correlation parameter. A more detailed analysis of the spatial correlation in the SCM channel can be found in [183]. A backward compatible extension to the SCM, known as SCME (SCM-Extended), was presented in [184]. The main contribution of this extension is an increase of the 
Table 2.2: Environment parameters in SCM specifications (see [182] for more details).

\begin{tabular}{|c|c|c|c|}
\hline Parameter & $\begin{array}{l}\text { Suburban } \\
\text { macrocell }\end{array}$ & $\begin{array}{l}\text { Urban } \\
\text { macrocell }\end{array}$ & Urban microcell \\
\hline Mean AS at BS & $5^{\circ}$ & $8^{\circ}, 15^{\circ}$ & $19^{\circ}$ \\
\hline $\begin{array}{l}\text { Per-path AS at BS } \\
\text { (fixed) }\end{array}$ & $2^{\circ}$ & $2^{\circ}$ & $5^{\circ}$ \\
\hline Mean AS at MS & $68^{\circ}$ & $68^{\circ}$ & $68^{\circ}$ \\
\hline $\begin{array}{l}\text { Per-path AS at MS } \\
\text { (fixed) }\end{array}$ & $35^{\circ}$ & $35^{\circ}$ & $35^{\circ}$ \\
\hline Mean total RMS DS & $0.17 \mu s$ & $0.65 \mu s$ & $0.251 \mu s$ \\
\hline $\begin{array}{l}\text { Log-normal shadow- } \\
\text { ing SD }\end{array}$ & $8 \mathrm{~dB}$ & $8 \mathrm{~dB}$ & $\begin{array}{l}\text { NLOS: } 10 \mathrm{~dB} \\
\text { LOS: } 4 \mathrm{~dB}\end{array}$ \\
\hline Path loss model & $\begin{array}{l}31.5+ \\
35 \log _{10}(d)\end{array}$ & $\begin{array}{l}34.5+ \\
35 \log _{10}(d)\end{array}$ & $\begin{array}{l}\text { NLOS: } 34.53+38 \log _{10}(d) \\
\text { LOS: } 30.18+26 \log _{10}(d)\end{array}$ \\
\hline
\end{tabular}

channel model bandwidth from $5 \mathrm{MHz}$ to $100 \mathrm{MHz}$. A comparison between these two channel models and the WINNER channel model is carried out in [185].

\section{$2.7 \quad$ Lattices}

The concept of lattices has been used in many different fields of mathematics, such as geometry, algebra, number theory or group theory, and many fields of engineering, such as in signal processing for wireless communications, image processing or cryptography. In this section, a brief overview of lattices and the problem of LR is presented. A more detailed review of LR can be found in [186-188].

\subsubsection{Introduction}

A real-valued lattice $\mathcal{L}$ is a discrete additive subgroup of the real Euclidean space $\mathbb{R}^{n}$. Any lattice can be characterized by a set of $m$ linearly independent vectors $\mathbf{b}_{1}, \ldots, \mathbf{b}_{m} \in \mathcal{L}$ as

$$
\mathcal{L}(\mathbf{B})=\left\{\mathbf{B z}, \mathbf{z} \in \mathbb{Z}^{m}\right\}=\left\{\sum_{i=1}^{m} z_{i} \mathbf{b}_{i}, z_{i} \in \mathbb{Z}\right\},
$$

where $\mathbf{B}=\left[\mathbf{b}_{1}, \ldots, \mathbf{b}_{m}\right]$ is the basis matrix of the lattice. The integers $n$ and $m \leq n$ are called the dimension and the rank of the lattice, respectively. If $m=n$, the lattice is called a "full-rank" lattice [188]. 
Two important features of a lattice are the fundamental parallelotope and the Voronoi region. The fundamental parallelotope of the lattice (a.k.a. fundamental region) consist of the set of points that can be written as

$$
\mathcal{P}(\mathbf{B})=\sum_{i=1}^{m} a_{i} \mathbf{b}_{i}, 0 \leq a_{i}<1 .
$$

Since the lattice basis is not unique, the fundamental parallelotope is not unique either. However, the volume of the different fundamental parallelotopes is the same for all the basis of a given lattice, and is given by

$$
|\mathcal{L}|=\sqrt{\operatorname{det}\left(\mathbf{B}^{T} \mathbf{B}\right)}
$$

The Voronoi region is defined as the set of points that are closer to the origin than to any other lattice point. This region can be expressed as

$$
\mathcal{V}(\mathcal{L})=\{\mathbf{x} \mid\|\mathbf{x}\| \leq\|\mathbf{x}-\mathbf{y}\| \forall \mathbf{y} \in \mathcal{L}\}
$$

Unlike the fundamental parallelotope, the Voronoi region is independent of the lattice basis. Figures 2.10 and 2.11 show the fundamental parallelotope and the Voronoi regions of some two-dimensional real-valued lattices.

The "quality" of a lattice basis can be obtained in terms of the orthogonality defect, defined as [186]

$$
\xi(\mathbf{B})=\frac{1}{|\mathcal{L}|} \prod_{i=1}^{m}\left\|\mathbf{b}_{i}\right\| .
$$

The orthogonality defect fulfills $\xi(\mathbf{B}) \geq 1$, with equality if and only if $\mathbf{B}$ is orthogonal. The condition number is also used to measure the "quality" of a lattice basis and can be calculated as

$$
\kappa(\mathbf{B})=\|\mathbf{B}\|\left\|\mathbf{B}^{-1}\right\|=\frac{\sigma_{\max }}{\sigma_{\min }},
$$

where $\sigma_{\max }$ and $\sigma_{\min }$ are the maximum and the minimum singular value of $\mathbf{B}$, respectively.

The previous definition of lattice can be straightforwardly generalized to the complex case as

$$
\mathcal{L}(\mathbf{B})=\left\{\mathbf{B z}, \mathbf{z} \in \mathbb{Z}_{j}^{m}\right\}=\left\{\sum_{i=1}^{m} z_{i} \mathbf{b}_{i}, z_{i} \in \mathbb{Z}_{j}\right\},
$$

where $\mathbf{b}_{i} \in \mathbb{C}^{n}, \mathbb{Z}_{j}^{m}=\mathbb{Z}^{m}+j \mathbb{Z}^{m}$ and $\mathbb{Z}_{j}=\mathbb{Z}+j \mathbb{Z}$ denoting sets of Gaussian integers. Since the matrix product $\mathbf{x}=\mathbf{B} \mathbf{z}$ can be expressed through its equivalent real-valued form,

$$
\left[\begin{array}{c}
\mathfrak{R e}\{\mathbf{x}\} \\
\mathfrak{I m}\{\mathbf{x}\}
\end{array}\right]=\left[\begin{array}{cc}
\mathfrak{R e}\{\mathbf{B}\} & -\mathfrak{I m}\{\mathbf{B}\} \\
\mathfrak{I m}\{\mathbf{B}\} & \mathfrak{R e}\{\mathbf{B}\}
\end{array}\right]\left[\begin{array}{c}
\mathfrak{R e}\{\mathbf{z}\} \\
\mathfrak{I m}\{\mathbf{z}\}
\end{array}\right]
$$




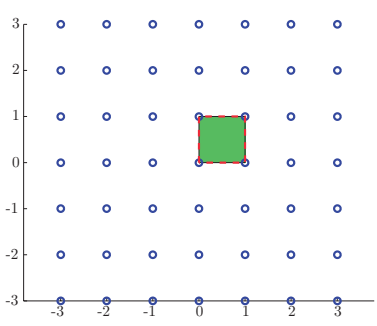

(a) $\mathbf{B}=\left(\begin{array}{ll}1 & 0 \\ 0 & 1\end{array}\right)$

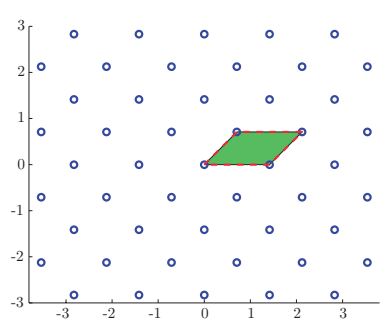

(b) $\mathbf{B}=\frac{1}{\sqrt{2}}\left(\begin{array}{ll}2 & 1 \\ 0 & 1\end{array}\right)$

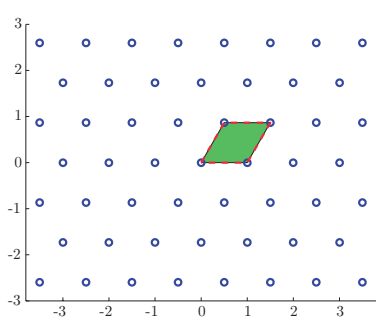

(c) $\mathbf{B}=\frac{1}{2}\left(\begin{array}{rr}2 & 1 \\ 0 & \sqrt{3}\end{array}\right)$

Figure 2.10: Illustration of two-dimensional example lattices showing their fundamental parallelotope.

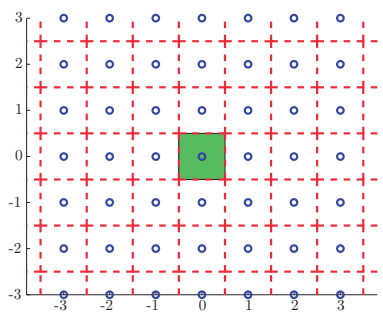

(a) $\mathbf{B}=\left(\begin{array}{ll}1 & 0 \\ 0 & 1\end{array}\right)$

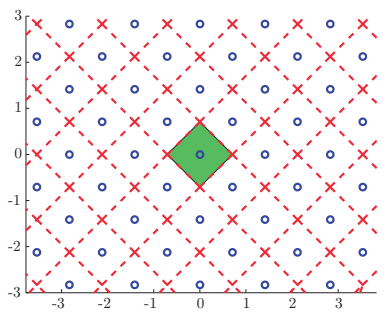

(b) $\mathbf{B}=\frac{1}{\sqrt{2}}\left(\begin{array}{ll}2 & 1 \\ 0 & 1\end{array}\right)$

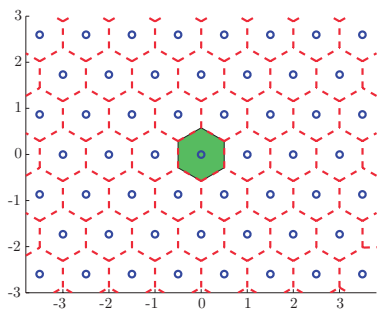

(c) $\mathbf{B}=\frac{1}{2}\left(\begin{array}{rr}2 & 1 \\ 0 & \sqrt{3}\end{array}\right)$

Figure 2.11: Illustration of two-dimensional example lattices showing their Voronoi region.

any $m$-dimensional complex-valued lattice in $\mathbb{C}^{n}$ can be expressed using a $2 m$ dimensional real-valued lattice in $\mathbb{R}^{2 n}[186]$.

\subsubsection{Lattice reduction}

The problem of LR has become very important in many fields of engineering, such as in signal processing for communications or cryptography.

Given a basis, LR consists of finding another basis whose vectors are more orthogonal and shorter, according to the Euclidean norm, than the original ones. The Minkowski or Hermite-Korkine-Zolotareff reductions are the techniques that obtain the best performance in terms of reduction, but also the ones with a higher computational cost [189]. Both techniques require the calculation of the shortest lattice vector, which has been proven to be a NP-hard problem (see [186], [187, Chap. 14], [190, Chap. 5] and references therein). 
In order to reduce the complexity of LR techniques, a polynomial computing time algorithm was proposed by Lenstra, Lenstra and Lovász, known as the LLL algorithm [14]. This algorithm, which can be seen as an extension of Gauss reduction [186] or a relaxation of Hermite-Korkine-Zolotareff conditions [188], obtains the reduced basis by applying two different operations over the original basis: size-reduction (or linear combination between columns) and column swap. Although some other reduction techniques have been proposed afterwards, the LLL algorithm is used the most due to the good trade-off between performance and computational complexity.

\section{LLL algorithm}

Let $\mathbf{B}^{*}=\left(\mathbf{b}_{1}^{*}, \ldots, \mathbf{b}_{m}^{*}\right) \in \mathbb{R}^{n \times m}$ denote the associated orthogonal basis of $\mathbf{B}$ calculated by the Gram-Schmidt orthogonalization (GSO) process as

$$
\begin{aligned}
& \mathbf{b}_{1}^{*}=\mathbf{b}_{1}, \\
& \mathbf{b}_{i}^{*}=\mathbf{b}_{i}-\sum_{j=1}^{i-1} \mu_{i, j} \mathbf{b}_{j}^{*} \quad \text { for } 2 \leq i \leq m,
\end{aligned}
$$

where

$$
\mu_{i, j}=\frac{\left(\mathbf{b}_{i}, \mathbf{b}_{j}^{*}\right)}{\left\|\mathbf{b}_{j}^{*}\right\|^{2}} \quad \text { for } 1 \leq j<i \leq n
$$

are known as the Gram-Schmidt coefficient (GSC). Thus, matrix B can be expressed as

$$
\mathbf{B}=\mathbf{B}^{*} \cdot \mathbf{U}^{T},
$$

where matrix $\mathbf{U}=\left[\mu_{i, j}\right] \in \mathbb{R}^{m \times m}$ is lower triangular with unit diagonal.

Definition 1 Given a lattice $\mathcal{L}$ with basis $\mathbf{B} \in \mathbb{R}^{n \times m}$, associated orthogonal basis $\mathbf{B}^{*} \in \mathbb{R}^{n \times n}$ and Gram-Schmidt coefficients $\mu_{i, j}, \mathbf{B}$ is called LLL-reduced if the following conditions are satisfied [14]:

$$
\begin{aligned}
\left|\mu_{i, j}\right| & \leq \frac{1}{2} & & \text { for } 1 \leq j<i \leq n \\
\left\|\mathbf{b}_{i}^{*}+\mu_{i, i-1} \mathbf{b}_{i-1}^{*}\right\|^{2} & \geq \delta\left\|\mathbf{b}_{i-1}^{*}\right\|^{2} & & \text { for } 1<i \leq n, \frac{1}{4}<\delta<1
\end{aligned}
$$

Condition (2.33) can be equivalently expressed as:

$$
\left\|\mathbf{b}_{i}^{*}\right\|^{2} \geq\left(\delta-\mu_{i, i-1}^{2}\right)\left\|\mathbf{b}_{i-1}^{*}\right\|^{2} \quad \text { for } 1<i \leq n, \frac{1}{4}<\delta<1
$$

The value of the constant $\delta$ in (2.33) and (2.34) may affect to the "quality" of the reduced basis and the computational complexity of the LLL algorithm. The 
typical value is $\delta=3 / 4$, which was the value used in [14]. Although $\delta=1$ is the value that obtains the lattice with the best orthogonal properties, it is not often utilized because the algorithm might not run in polynomial time [191, Chap. 2].

The original LLL algorithm is shown in Algorithm 1. The input of the algorithm consists of the matrix containing the basis vectors, $\mathbf{B}=\left[\mathbf{b}_{1}, \ldots, \mathbf{b}_{m}\right]$, and the reduction parameter $\delta$. In this "in-place" version of the LLL algorithm, the original basis $\mathbf{B}$ is overwritten by the reduced basis $\widetilde{\mathbf{B}}$. The transformations performed on matrix $\mathbf{B}$ are registered in the transformation matrix $\mathbf{T}$, so that

$$
\widetilde{\mathbf{B}}=\mathbf{B T}
$$

where $\mathbf{T}$ is an unimodular matrix, $|\operatorname{det}(\mathbf{T})|=1$, with integer elements.

Two different procedures are used in the LLL algorithm: $\operatorname{SizeReduction}(k, l)$ and $\operatorname{SwaP}(k)$. Procedure $\operatorname{SizeReduction}(k, l)$ checks for the condition (2.32). If the condition is fulfilled, the procedure does nothing. Otherwise, the procedure reduces $\mathbf{b}_{k}$ by subtracting integer $\mu$ times $\mathbf{b}_{l}$. The reason of rounding $\mu_{k, l}$ to the closest integer $\mu$ is to assure that the new $\mathbf{b}_{k}$ remains in the lattice. Then, the GSC are updated. Procedure $\operatorname{SwAP}(k)$ swaps the vectors $\mathbf{b}_{k}$ and $\mathbf{b}_{k-1}$ and updates the orthogonal basis and the GSC.

In the main loop of the algorithm, size reductions are applied over contiguous vectors. Then, if condition (2.34) is fulfilled, size reductions are applied between the current vector and the previous ones and the index $k$ is increased. If the condition is not met, a vector swap is carried out and $k$ is decreased. The termination of the algorithm is not straightforward, but it has been proven that the LLL runs in a polynomial time. More details about the algorithm ant its implementation can be found in $[14,187,188,191]$. 


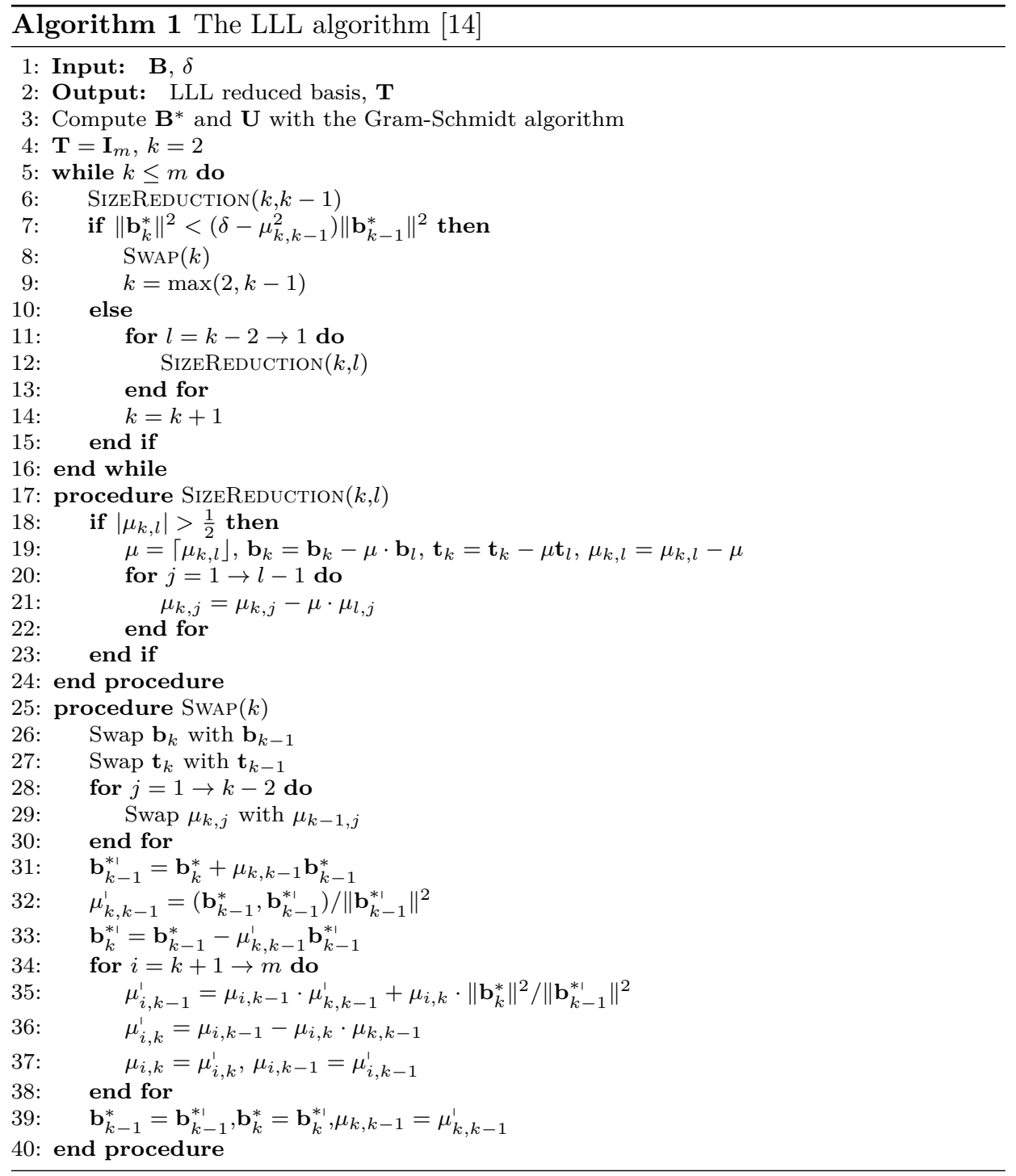


Part I

Precoding 



\section{Chapter 3}

\section{Precoding with Perfect CSIT}

This chapter contains the description of several sub-optimal multiuser precoding techniques to allow multiuser spatial multiplexing. The techniques analyzed include SE, ZF, THP and techniques based on LR. The performance and the computational cost of the different techniques presented in [192] are shown.

In the last decades, different precoding techniques allowing spatial multiplexing of several users have been proposed to improve the spectral efficiency of multiuser multiple-input multiple-output (MU-MIMO) communication systems. DPC is a theoretical scheme that allows the precancellation of the non-casually known interference at the transmitter without incurring a power penalty [40]. For a given user ordering, DPC is serially applied over the users allowing to pre-subtract the interference caused by users with lower indices [26]. Although it has been proven that DPC achieves the whole capacity region of the MIMO broadcast channel [41-44], it suffers from a high level of complexity. In practical systems, precoding schemes requiring lower complexity are usually utilized.

This thesis focuses on the downlink of MU-MIMO systems with single-antenna MSs, a.k.a. MU-MISO. The received signal at the $m$ th MS has been expressed in (2.10). However, this equation can be expressed in a more compact way by aggregating the received signal of the different users in a vector as

$$
\mathbf{y}=\mathbf{H} \mathbf{x}+\mathbf{n},
$$

where $\mathbf{y} \in \mathbb{C}^{M \times 1}$ contains the received signal for the $M$ MSs, $\mathbf{H}=\left[\mathbf{h}_{1} \ldots \mathbf{h}_{M}\right]^{T} \in$ $\mathbb{C}^{M \times N_{t}}$ is the aggregated downlink channel matrix, $\mathbf{x} \in \mathbb{C}^{N_{t} \times 1}$ represents the transmitted signal and $\mathbf{n} \in \mathbb{C}^{M \times 1}$ is the noise vector whose elements are i.i.d. circularly-symmetric complex Gaussian random variables, with zero mean and 
unit variance, $\mathcal{C N}(0,1)$. It is important to note that this notation can also be extended to a coordinated system as the one shown in (2.14).

Similarly to (2.27), the received signal in (3.1) can be expressed through its realvalued form as

$$
\left[\begin{array}{c}
\mathfrak{R e}\{\mathbf{y}\} \\
\mathfrak{I m}\{\mathbf{y}\}
\end{array}\right]=\left[\begin{array}{cc}
\mathfrak{R e}\{\mathbf{H}\} & -\mathfrak{I m}\{\mathbf{H}\} \\
\mathfrak{I m}\{\mathbf{H}\} & \mathfrak{R e}\{\mathbf{H}\}
\end{array}\right]\left[\begin{array}{c}
\mathfrak{R e}\{\mathbf{x}\} \\
\mathfrak{I m}\{\mathbf{x}\}
\end{array}\right]+\left[\begin{array}{c}
\mathfrak{R e}\{\mathbf{n}\} \\
\mathfrak{I m}\{\mathbf{n}\}
\end{array}\right]
$$

obtaining a $\left(2 M \times 2 N_{t}\right)$-dimensional real representation. This model, which can be more convenient for practical implementations or when working with lattices, will be denoted as

$$
\mathbf{y}_{\mathrm{r}}=\mathbf{H}_{\mathrm{r}} \mathbf{x}_{\mathrm{r}}+\mathbf{n}_{\mathrm{r}}
$$

An interesting sub-optimal technique is vector-perturbation (VP), which can be considered as a general form of precoding. This technique consists of perturbing the data in order to reduce the power of the transmitted signal [22] or to maximize the SNR in case the transmit power is fixed. The precoded signal can be expressed as

$$
\mathbf{x}=\sqrt{\gamma \mathbf{H}^{\dagger}}(\mathbf{s}+\mathbf{p})
$$

where $\mathbf{s} \in \mathbb{C}^{M \times 1}$ is the vector which contains the original data symbols of the $M$ users, $\mathbf{p} \in \mathbb{C}^{M \times 1}$ denotes de perturbation vector and $\sqrt{\gamma}$ limits the sum-power of all the transmit antennas, $\|\mathbf{x}\|^{2}$. Since the actual transmit power depends on the data $\mathbf{s}, \sqrt{\gamma}$ is usually chosen to assure a certain average sum-power $\mathbb{E}\left[\|\mathbf{x}\|^{2}\right]=P$. Using the real-valued equivalent model, the precoded signal is given by

$$
\mathbf{x}_{\mathrm{r}}=\sqrt{\gamma} \mathbf{H}_{\mathrm{r}}^{\dagger}\left(\mathbf{s}_{\mathrm{r}}+\mathbf{p}_{\mathrm{r}}\right) \text {. }
$$

The perturbation vector cannot be an arbitrary vector since this perturbation is unknown at the MSs and will cause decoding errors. In order to allow the MSs to remove the perturbation, the perturbation is set to

$$
\mathbf{p}=A \mathbf{l}
$$

where $A$ is a positive real number and $\mathbf{l}$ is an $M$-dimensional complex vector $\mathbf{l}_{\mathrm{R}}+j \mathbf{l}_{\mathrm{I}}$, where $\mathbf{l}_{\mathrm{R}}, \mathbf{l}_{\mathrm{I}} \in \mathbb{Z}^{M}$. Equivalently,

$$
\mathbf{p}_{\mathrm{r}}=A \mathbf{l}_{\mathrm{r}},
$$

where $\mathbf{l}_{\mathrm{r}} \in \mathbb{Z}^{2 M}$. The value of $A$ is chosen such that the points from the signal constellation can be uniquely recovered. Assuming an $\mathcal{M}$-QAM modulation whose components take odd integer values and its average power is given by

$$
\sigma_{s}^{2}=\frac{2(\mathcal{M}-1)}{3}
$$




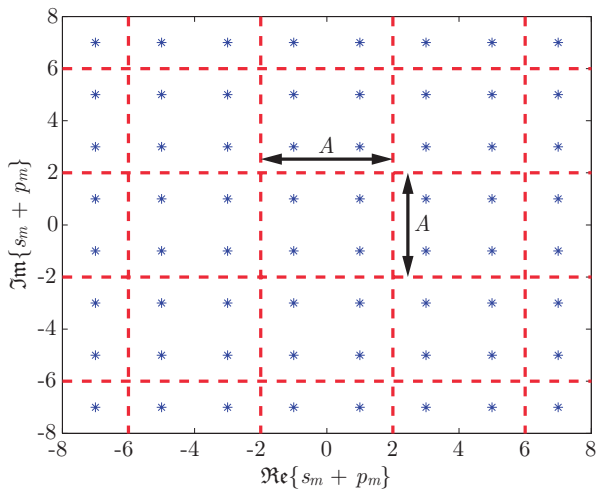

(a) $\mathcal{M}=4$

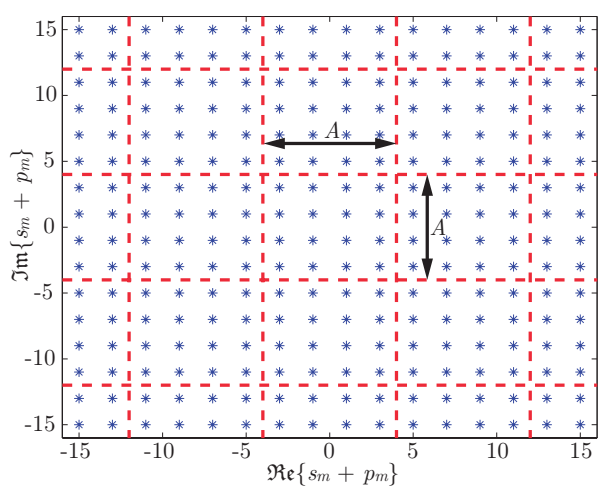

(b) $\mathcal{M}=16$

Figure 3.1: Illustration of the effect of the perturbation in square $\mathcal{M}$-QAM modulated signals with $A=2 \sqrt{\mathcal{M}}$.

a possible value of $A$ is given by $A=2 \sqrt{\mathcal{M}}$. Larger values of $A$ reduce the effect of the perturbation, leading to $\mathbf{l}=\mathbf{0}$ as $A$ increases. A more detailed discussion about the value of $A$ can be found in [22]. The MSs remove the perturbation applying the modulo operation:

$$
y_{m} \operatorname{MOD} A:=y_{m}-A\left\lfloor\frac{y+A / 2}{A}\right\rfloor .
$$

In the complex-valued model, the modulo operation is applied to both the real and imaginary parts separately. Figure 3.1 shows the effect of the perturbation in square $\mathcal{M}$-QAM modulated signals. It can be seen that the original constellation is extended periodically and the modulo operation allows to bring any symbol back to its equivalent symbol in the original constellation.

There are different techniques to obtain the perturbation vector which differ in performance and computational complexity: sphere encoder (SE) [22], zero-forcing (ZF) [20], Tomlinson-Harashima precoding (THP) [21] or LR-based techniques [15, 16]. These techniques can also be used for multiple-antenna receivers, making the detection easier since the interference is cancelled at each antenna. However, a higher throughput can be achieved by block diagonalization (BD). This technique consists of only cancelling the multiuser interference, allowing the interference between the different spatial streams of each user, which in turn can be cancelled through detection techniques thanks to the use of multiple antennas at the receiver [47]. 


\subsection{Zero-Forcing}

Channel inversion is known as ZF precoding when it is performed at the transmitter. This linear technique was proposed for single-antenna MSs and suffers from a power enhancement when the channel matrix is ill-conditioned [20]. It can be considered as a specific case of VP where $\mathbf{p}=\mathbf{0}$, so the modulo operation is not required at the receiver. The beamforming matrix is directly obtained from the Moore-Penrose pseudoinverse and the precoded signal can be expressed as (Fig. 3.2)

$$
\mathbf{x}=\sqrt{\gamma_{\mathrm{ZF}} \mathbf{H}^{\dagger} \mathbf{s}}
$$

where $\sqrt{\gamma_{\mathrm{ZF}}}$ limits the average sum-power $\mathbb{E}\left[\|\mathbf{x}\|^{2}\right]=P$ and can be obtained from

$$
\gamma_{\mathrm{ZF}}=\frac{P / \sigma_{s}^{2}}{\operatorname{Tr}\left(\left(\mathbf{H H}^{H}\right)^{-1}\right)}=\frac{P / \sigma_{s}^{2}}{\sum_{m=1}^{M}\left(1 / \lambda_{m}^{2}\right)},
$$

where $\lambda_{m}$ is the $m$ th singular value of $\mathbf{H}$. Other possible values of $\gamma_{\mathrm{ZF}}$ are analyzed in [193]. In addition, more realistic power constraints such as per-antenna power constraints or per-BS power constraints in coordinated networks have been analyzed in [194] and [61], respectively. The received signal by the MSs can be expressed as

$$
\mathbf{y}=\mathbf{H} \mathbf{x}+\mathbf{n}=\sqrt{\gamma_{\mathrm{ZF}} \mathbf{H H}^{\dagger} \mathbf{s}}+\mathbf{n}=\sqrt{\gamma_{\mathrm{ZF}}} \mathbf{s}+\mathbf{n} .
$$

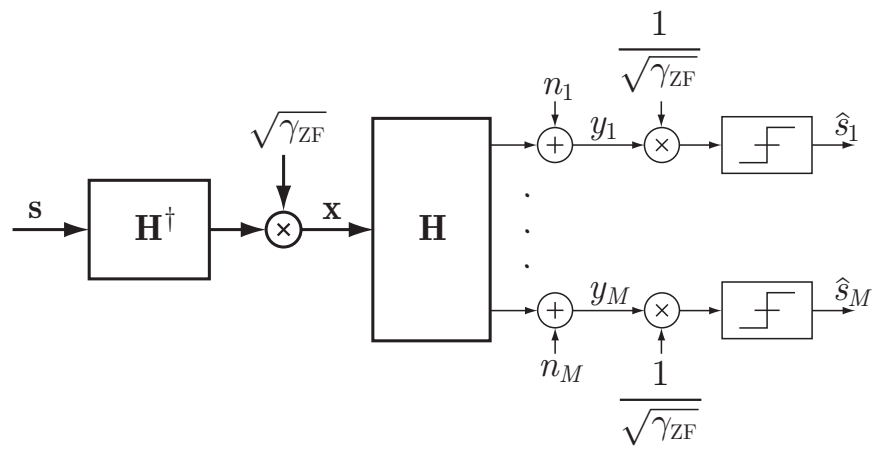

Figure 3.2: ZF precoding scheme.

It can be seen that all the MSs receive the original signal scaled by the same value and contaminated with additive noise. Thus, all the MSs experience the same SNR, $\gamma_{\mathrm{ZF}} \sigma_{s}^{2}$, and hence achieve the same rate. The system sum-rate can be expressed as

$$
R_{\mathrm{ZF}}=M \log _{2}\left(1+\gamma_{\mathrm{ZF}} \sigma_{s}^{2}\right)=M \log _{2}\left(1+\frac{P}{\sum_{m=1}^{M}\left(1 / \lambda_{m}^{2}\right)}\right)
$$


This scheme assures the same rate for the different MSs and guarantees the minimization of the maximum BER [195]. However, different power allocation among the MSs have been proposed in order to maximize the sum-rate or minimize the sum-BER [194-196].

\subsection{Sphere encoder}

The optimal choice of the perturbation vector $\mathbf{p}_{\mathrm{r}}$ is such that it minimizes the power of the transmitted signal [22]:

$$
\mathbf{p}_{\mathrm{r}}=\arg \min _{\mathbf{p}_{\mathrm{r}}^{\prime} \in A \mathbb{Z}^{2 M}}\left\|\mathbf{H}_{\mathrm{r}}^{\dagger}\left(\mathbf{s}_{\mathrm{r}}+\mathbf{p}_{\mathrm{r}}^{\prime}\right)\right\|^{2} .
$$

Restricting the possible perturbation to a limited range, the optimal perturbation can be found by exhaustive search over all the equivalent symbols in the extended constellation (Fig. 3.1). However, note that (3.14) can be seen as a search for the point $\mathbf{H}_{\mathrm{r}}^{\dagger} \mathbf{p}_{\mathrm{r}}^{\prime}$ that is closest to $-\mathbf{H}_{\mathrm{r}}^{\dagger} \mathbf{s}_{\mathrm{r}}$, which is a search for a $2 M$-dimensional lattice point. The solution to this problem can be obtained more efficiently using the Fincke and Pohst algorithm [197-199]. Since this algorithm is known as sphere decoder when it is used for space-time demodulation in [200], it is often referred to as sphere encoder when applied in precoding. This search can also be computationally expensive and therefore, other alternative approaches are usually employed.

The precoded signal for the SE scheme is expressed as ${ }^{1}$

$$
\mathbf{x}=\sqrt{\gamma_{\mathrm{SE}} \mathbf{H}^{\dagger}}(\mathbf{s}+\mathbf{p}),
$$

and the received signal as

$$
\begin{aligned}
\mathbf{y} & =\mathbf{H} \mathbf{x}+\mathbf{n}=\sqrt{\gamma_{\mathrm{SE}}} \mathbf{H H}^{\dagger}(\mathbf{s}+\mathbf{p})+\mathbf{n}= \\
& =\sqrt{\gamma_{\mathrm{SE}}}(\mathbf{s}+\mathbf{p})+\mathbf{n} .
\end{aligned}
$$

Since the perturbation $\mathbf{p}$ is removed with the modulo operation (see Fig. 3.3), the SNR experienced by the MSs is $\gamma_{\mathrm{SE}} \sigma_{s}^{2}$ and the system sum-rate is given by

$$
R_{\mathrm{SE}}=M \log _{2}\left(1+\gamma_{\mathrm{SE}} \sigma_{s}^{2}\right) .
$$

\footnotetext{
${ }^{1}$ Note that the real-valued equivalent model has been used to obtain the optimal perturbation in (3.14) and once the perturbation is obtained, the complex-valued model is used for the sake of comparison between the different algorithms.
} 


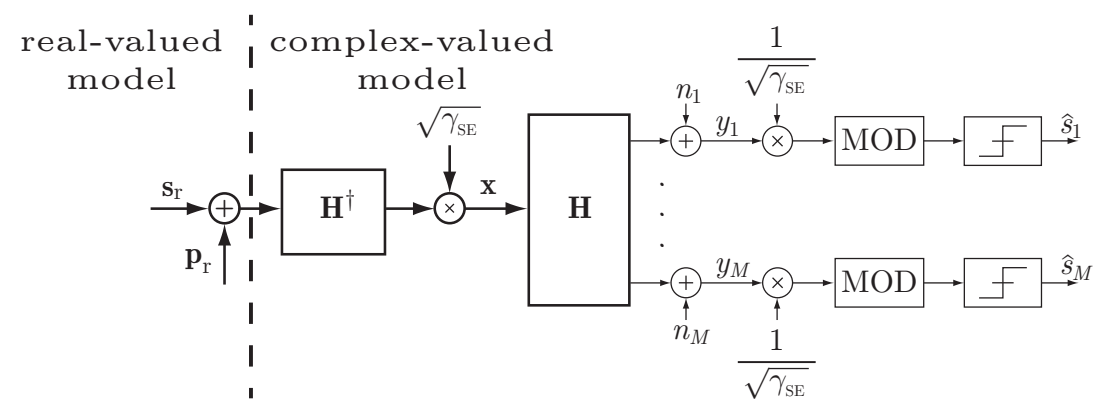

Figure 3.3: SE scheme.

\subsection{Tomlinson-Harashima precoding}

The main drawback of $\mathrm{ZF}$ is that it requires a high transmit power to ensure a certain QoS when the channel matrix is ill-conditioned. THP makes use of the modulo operator to reduce the power of the transmitted signal compared to a linear precoding scheme [21]. A detailed analysis of THP for the SU-MIMO case is carried out in [201-203]. In [201], it is shown that THP is equivalent to $\mathrm{VP}$ where the perturbation vector $\mathbf{p}$ is obtained sequentially but very efficiently through the feedback filter and the modulo operation. The perturbation of the transmitted signal reduces its power and allows cancelation of the interference when the modulo operator is applied at the receiver. Figure 3.4 shows the THP scheme for decentralized receivers [204].

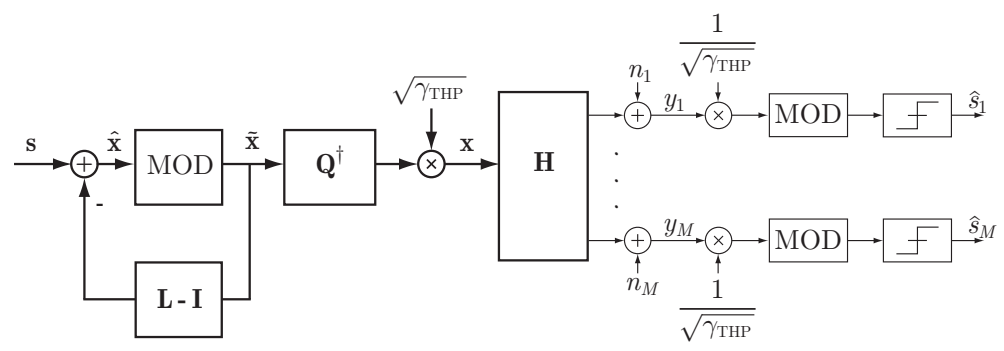

Figure 3.4: THP scheme for decentralized receivers.

The matrices that take part in the precoding process are obtained from an LQ decomposition of the channel matrix,

$$
\mathbf{H}=\mathbf{L Q},
$$


where $\mathbf{L} \in \mathbb{C}^{M \times M}$ is a lower triangular matrix with ones in its diagonal and $\mathbf{Q} \in \mathbb{C}^{M \times N_{t}}$ contains orthogonal rows. Matrices $\mathbf{L}$ and $\mathbf{Q}$ can be expressed as

$$
\begin{aligned}
\mathbf{L} & =\mathbf{L}_{0} \mathbf{G}^{-1} \\
\mathbf{Q} & =\mathbf{G Q}_{0},
\end{aligned}
$$

where $\mathbf{L}_{0}$ and $\mathbf{Q}_{0}$ are obtained from a conventional LQ decomposition, $\mathbf{H}=\mathbf{L}_{0} \mathbf{Q}_{0}$, and $\mathbf{G}$ is a diagonal matrix containing the diagonal of $\mathbf{L}_{0}$.

It can be noted that, if the modulo operation were omitted, this scheme would be equivalent to ZF. However, using modulo operation at the transmitter allows the reduction of the transmit power by reducing the symbols in $\tilde{\mathbf{x}}$ into the boundary region of the $\mathcal{M}$-QAM constellation. The precoded symbols $\hat{\mathbf{x}}$ can be initially expressed as

$$
\hat{x}_{m}=s_{m}-\sum_{l=1}^{m-1} l_{m, l} \tilde{x}_{m} .
$$

Next, the modulo operation restricts the symbols to the original constellation,

$$
\tilde{x}_{m}=\hat{x}_{m} \operatorname{MOD} A,
$$

and thus the transmit power of this method is lower than the linear precoding. The modulo operation can be modeled by adding integer multiples of $A$ to the real and imaginary parts of the original signal before the linear filtering,

$$
\tilde{\mathbf{x}}=\mathbf{L}^{-1}(\mathbf{s}+\mathbf{p}),
$$

Finally, $\mathbf{x}=\sqrt{\gamma_{\mathrm{THP}}} \mathbf{Q}^{\dagger} \tilde{\mathbf{x}}$ is transmitted over the channel. The transmitted signal can be expressed as

$$
\mathbf{x}=\sqrt{\gamma_{\mathrm{THP}}} \mathbf{Q}^{\dagger} \mathbf{L}^{-1}(\mathbf{s}+\mathbf{p}) .
$$

This expression is equivalent to (3.4), with the difference that $\mathbf{p}$ has not been explicitly calculated, but it has been obtained through the feedback filter and the modulo operation. Constant $\sqrt{\gamma_{\text {THP }}}$ is now determined by

$$
\gamma_{\mathrm{THP}}=\frac{\mathcal{M}-1}{\mathcal{M}} \frac{P / \sigma_{s}^{2}}{\sum_{m=1}^{M}\left(1 / l_{0, m m}^{2}\right)},
$$

where $l_{0, m m}$ represents the $m$ th element in the diagonal of $\mathbf{L}_{0}$ in (3.19). The first factor compensates the slight power increase of $\tilde{\mathbf{x}}$ with regard to s. Signal $\tilde{\mathbf{x}}$ is uniformly distributed over the boundary region of a $\mathcal{M}$-QAM modulated signal [205, Chap. 3]. However, this power increase is not very significant for high order modulation. The signal received by the users can be expressed as:

$$
\begin{aligned}
\mathbf{y} & =\mathbf{H} \mathbf{x}+\mathbf{n}=\sqrt{\gamma_{\mathrm{THP}} \mathbf{H} \mathbf{Q}^{\dagger} \mathbf{L}^{-1}}(\mathbf{s}+\mathbf{p})+\mathbf{n}= \\
& =\sqrt{\gamma_{\mathrm{THP}}}(\mathbf{s}+\mathbf{p})+\mathbf{n} .
\end{aligned}
$$


Disregarding the perturbation vector $\mathbf{p}$ since it is removed at the receiver with the modulo operation, it can be easily seen that all the MSs experience the same $\mathrm{SNR}, \gamma_{\mathrm{THP}} \sigma_{s}^{2}$. Thus, all the MSs achieve the same rate and the system sum-rate is expressed as

$$
\begin{aligned}
R_{\mathrm{THP}} & =M \log _{2}\left(1+\gamma_{\mathrm{THP}} \sigma_{s}^{2}\right) \\
& =M \log _{2}\left(1+\frac{\mathcal{M}-1}{\mathcal{M}} \frac{P}{\sum_{m=1}^{M}\left(1 / l_{0, m m}^{2}\right)}\right) .
\end{aligned}
$$

A more detailed analysis of the achievable rates and some other practical aspects such as the MSs ordering are presented in [206].

\subsection{Lattice-Reduction-aided precoding}

LR techniques have demonstrated to improve the detection stage of SU-MIMO communications [207, 208]. In addition, LR is known to be a very useful method for precoding in multiuser downlink communications, as various research on this topic shows $[15,16]$.

Although the complexity of (3.14) can be alleviated with the use of the Fincke and Pohst algorithm, its computational complexity is still high compared with ZF or THP. LRAP techniques make use of LR to obtain efficient approximations of (3.14). Two different LRAP techniques are presented in [15]: LRAP-linear and LRAP-VB. In addition, a scheme where THP is applied after performing a LR over the channel matrix is presented in [16].

\subsubsection{LRAP-linear}

This technique uses the rounding off approximation to solve (3.14) [209]. The approximated perturbation vector is obtained as [15]

$$
\mathbf{p}_{\mathrm{r}}=-A \mathbf{R}^{-1}\left\lceil\frac{\mathbf{R s}_{\mathrm{r}}}{A}\right\rfloor,
$$

where $\mathbf{R}$ is the transformation matrix obtained from an LR applied to the columns of $\mathbf{H}_{\mathrm{r}}^{\dagger}$ such that $|\operatorname{det}(\mathbf{R})|=1$ and

$$
\mathbf{H}_{\mathrm{r}}^{\dagger}=\widetilde{\mathbf{H}_{\mathrm{r}}^{\dagger}} \mathbf{R} .
$$

Here, $\widetilde{\mathbf{H}_{\mathrm{r}}^{\dagger}}$ is the reduced basis with better orthogonality properties.

The precoded signal can be directly obtained from (3.5). Figure 3.5 shows the LRAP-linear scheme. Similarly to the SE case, the system sum-rate can be expressed as

$$
R_{\mathrm{LRAP}-\operatorname{lin}}=M \log _{2}\left(1+\gamma_{\mathrm{LRAP}-\operatorname{lin}} \sigma_{s}^{2}\right)
$$




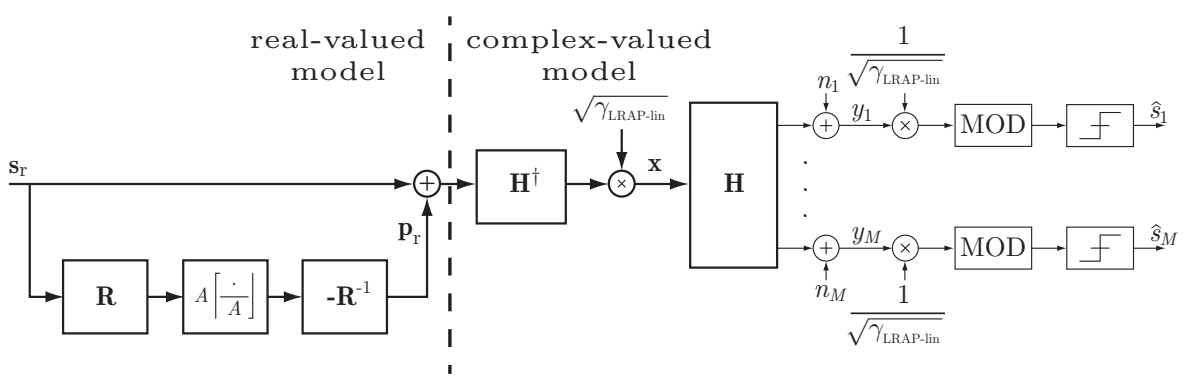

Figure 3.5: LRAP-linear scheme.

\subsubsection{LRAP-VB}

A variant of the nearest-plane algorithm is also considered for the solution of (3.14) in [15]. This algorithm is similar to successive interference cancellation (SIC) and consists of successive rounding operations considering the previous rounded values. Here the matrices $\mathbf{Q}$ and $\mathbf{L}$ can also be straightforwardly calculated from a QR-like decomposition of $\mathbf{H}_{\mathrm{r}}^{\dagger}$ such that:

$$
\widetilde{\mathrm{QH}} \widetilde{\mathrm{r}}^{\dagger}=\mathbf{L}
$$

where $\mathbf{Q}$ contains orthogonal rows and $\mathbf{L}$ is a lower triangular matrix. It can be observed in Fig. 3.6 that the first step of the algorithm builds the vector

$$
\mathbf{q}=-\mathbf{Q} \mathbf{H}_{\mathrm{r}}^{\dagger} \mathbf{s}_{\mathrm{r}} \text {. }
$$

Next, the components of $\widetilde{\mathbf{q}}$ are calculated as

$$
\widetilde{\mathrm{q}}_{m}=A\left\lceil\frac{\mathrm{q}_{m}-\sum_{l=1}^{m-1} \mathrm{~L}_{m, l} \widetilde{\mathrm{q}}_{l}}{A}\right\rfloor .
$$

Finally, the vector perturbation is calculated as

$$
\mathbf{p}_{\mathrm{r}}=\mathbf{R}^{-1} \widetilde{\mathbf{q}}
$$

Likewise, the precoded signal can be directly obtained from (3.5) and the system sum-rate can be expressed as

$$
R_{\mathrm{LRAP}-\mathrm{VB}}=M \log _{2}\left(1+\gamma_{\mathrm{LRAP}-\mathrm{VB}} \sigma_{s}^{2}\right) .
$$




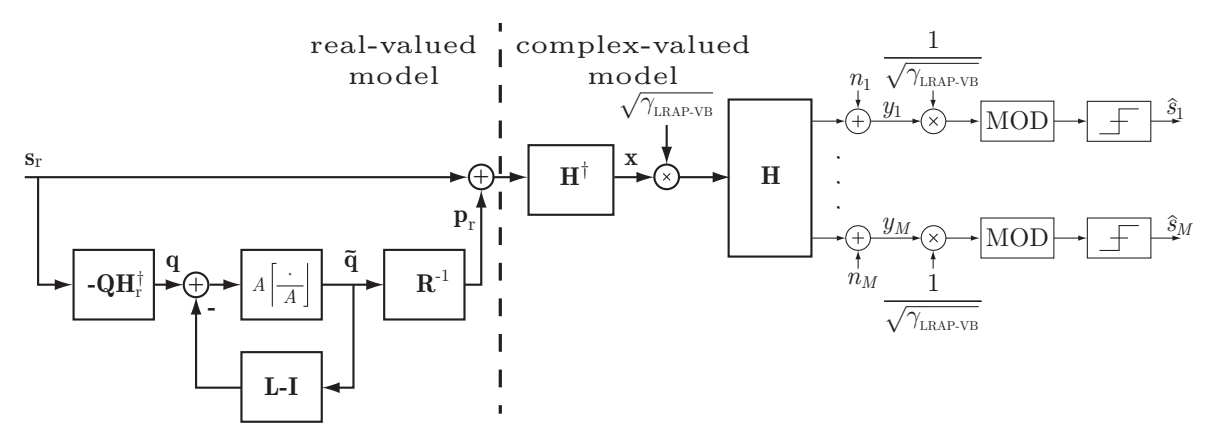

Figure 3.6: LRAP-VB scheme.

\subsubsection{LR-THP}

The THP strategy can also be performed after an LR of the channel matrix [16]. As shown in Fig. 3.7, the components of the original signal vector $\mathbf{s}$ in (3.21) are replaced by the components of a new vector

$$
\tilde{\mathbf{s}}=\mathbf{R}^{-1} \mathbf{s},
$$

where $\mathbf{R}$ is the transformation matrix obtained from an LR applied to the rows of $\mathbf{H}$ such that $|\operatorname{det}(\mathbf{R})|=1$ and

$$
\mathbf{H}=\mathbf{R} \widetilde{\mathbf{H}} .
$$

It is important to note that this LR process is slightly different from the one in $(3.29)$.

In this scheme, the THP is performed over the lattice-reduced channel matrix, which shows better orthogonality properties. Thus, matrices $\mathbf{L}$ and $\mathbf{Q}$ are obtained from the LQ decomposition of $\widetilde{\mathbf{H}}$,

$$
\widetilde{\mathbf{H}}=\mathbf{L} \mathbf{Q}=\left(\mathbf{L}_{0} \mathbf{G}^{-1}\right)\left(\mathbf{G} \mathbf{Q}_{0}\right),
$$

and constant $\sqrt{\gamma_{\mathrm{LR}-\mathrm{THP}}}$ can be calculated as

$$
\gamma_{\mathrm{LR}-\mathrm{THP}}=\frac{\mathcal{M}-1}{\mathcal{M}} \frac{P / \sigma_{s}^{2}}{\sum_{m=1}^{M}\left(1 / l_{0, m m}^{2}\right)},
$$

where $l_{0, m m}$ represents the $m$ th element in the diagonal of $\mathbf{L}_{0}$ in (3.38), whose calculation is identical to (3.19). It has been observed that the product $\mathbf{R}^{-1} \mathbf{s}$ does not change the average power of $\tilde{\mathbf{x}}$, which is given in [205, Chap. 3]. However, it is important to realize that the number of terms in the summation in (3.39) is $M$. Therefore, in order to obtain the constant $\gamma_{\mathrm{LR}-\mathrm{THP}}$ it is necessary to perform a complex LR in (3.37) such as the proposed in [210, 211]. 
The precoded signal can be expressed as

$$
\mathbf{x}=\sqrt{\gamma_{\mathrm{LR}-\mathrm{THP}}} \mathbf{Q}^{\dagger} \mathbf{L}^{-1}(\tilde{\mathbf{s}}+\mathbf{p}),
$$

and the received signal as

$$
\begin{aligned}
\mathbf{y} & =\mathbf{H} \mathbf{x}+\mathbf{n}=\sqrt{\gamma_{\mathrm{LR}-\mathrm{THP}} \mathbf{R} \tilde{\mathbf{H}} \mathbf{Q}^{\dagger} \mathbf{L}^{-1}(\tilde{\mathbf{s}}+\mathbf{p})+\mathbf{n}=} \\
& =\sqrt{\gamma_{\mathrm{LR}-\mathrm{THP}} \mathbf{R}(\tilde{\mathbf{s}}+\mathbf{p})+\mathbf{n}} .
\end{aligned}
$$

Since the elements of $\mathbf{R}$ are integers and the operations of multiplication and addition on integer sets are closed, the term $\mathbf{R} \mathbf{p}$ can be expressed as $\mathbf{R} \mathbf{p}=A \mathbf{l}$ where $A$ is a positive real number and $\mathbf{l}$ is an $M$-dimensional complex vector $\mathbf{l}_{\mathrm{R}}+j \mathbf{l}_{\mathrm{I}}$ with $\mathbf{l}_{\mathrm{R}}, \mathbf{l}_{\mathrm{I}} \in \mathbb{Z}^{M}$. Hence, the term $\mathbf{R p}$ can be removed at the receiver with the modulo operation.

Finally, the system sum-rate can be expressed as

$$
\begin{aligned}
R_{\mathrm{LR}-\mathrm{THP}} & =M \log _{2}\left(1+\gamma_{\mathrm{LR}-\mathrm{THP}} \sigma_{s}^{2}\right) \\
& =M \log _{2}\left(1+\frac{\mathcal{M}-1}{\mathcal{M}} \frac{P}{\sum_{m=1}^{M}\left(1 / l_{0, m m}^{2}\right)}\right) .
\end{aligned}
$$

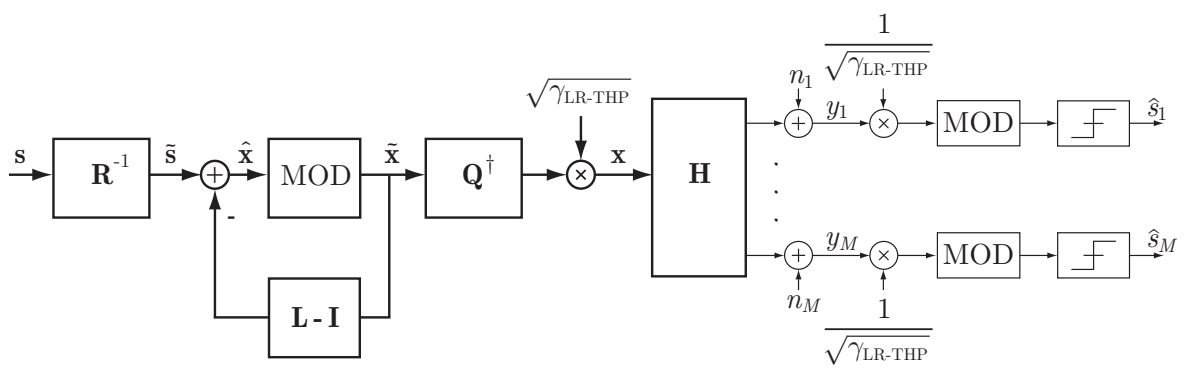

Figure 3.7: LR-THP scheme.

\subsection{Performance}

In this section, a performance comparison among the precoding algorithms under study is presented for the sake of completeness. The comparison has been carried out in terms of BER and sum-rate. A system such as the one presented in (3.1) is considered. A 4-QAM modulation has been used in all the simulations, while two different configurations regarding the number of transmit antennas and number of users have been considered: $N_{t}=M=4$ and $N_{t}=M=8$. The results are shown for a transmit power $P$ ranging from 0 to $30 \mathrm{~dB}$. 


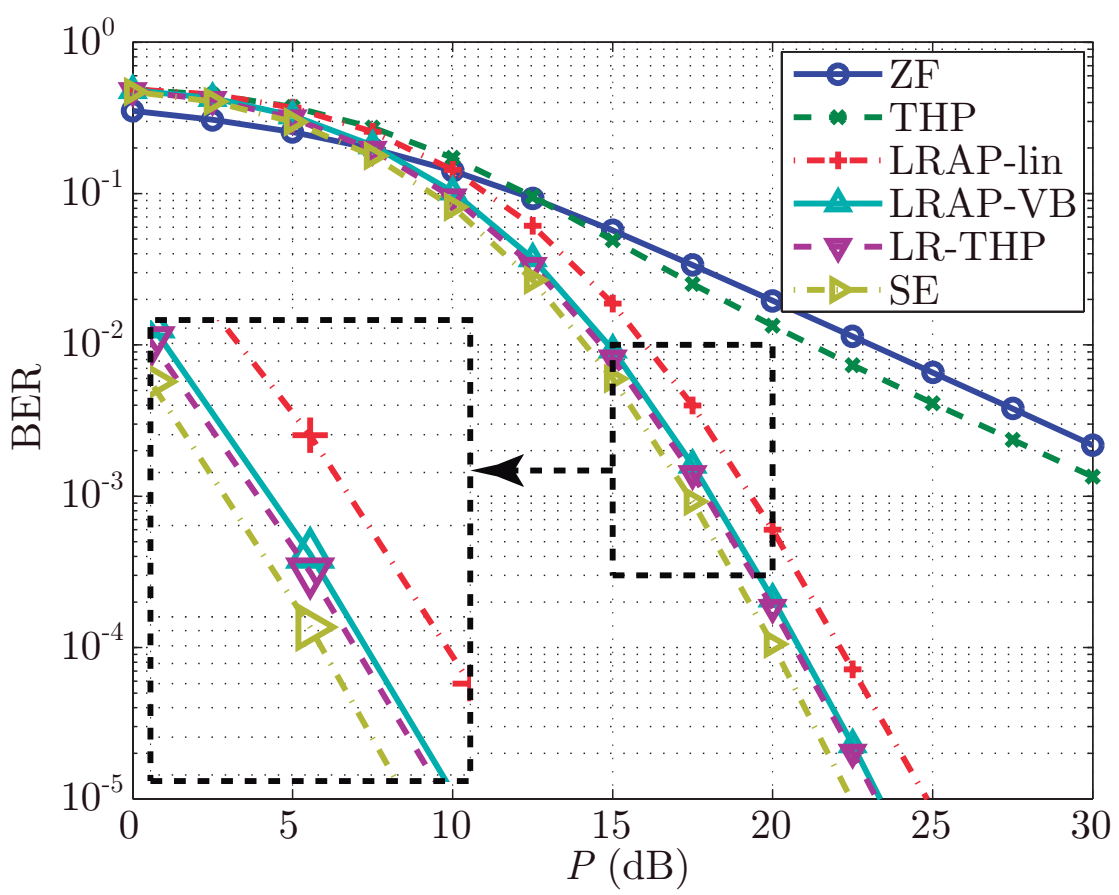

Figure 3.8: BER for the different precoding techniques for $N_{t}=M=4$ and 4-QAM.

It is important to note that, as highlighted in Sec. 4.1, the power allocation among the MSs assure the same SNR at each MS. However, other power allocations can maximize the sum-rate by allocating a higher power to those MSs that experience better channel conditions [194-196, 212], at the cost of reducing the rate and increasing the BER of those MSs with a worse channel. Likewise, the user-ordering can be optimized to further improve the sum-rate or BER [21, 206, 213, 214].

Figure 3.8 shows the BER for $N_{t}=M=4$. The diversity order is defined as the negative of the asymptotic slope of the average symbol error rate (SER) versus SNR in a log-log plot, i.e., the orders of magnitude gained by an increase of 10 dB [102, Chap. 1], [215, Chap. 7]. Since a 4-QAM modulation with Gray mapping is used, the SER for high $P$ can be approximated by SER $\approx 2$ BER. Thus, the slope in a log-log plot does not change and the diversity order can be obtained from the BER curve. On the one hand, results show that THP slightly outperforms ZF due to the integration of the modulo operation. However, both schemes show a diversity order equal to 1 . On the other hand, results show that SE and LR-based techniques show the full diversity order 4. LR-based techniques have shown a performance considerably close to SE, particularly LRAP-VB and LR-THP. The 


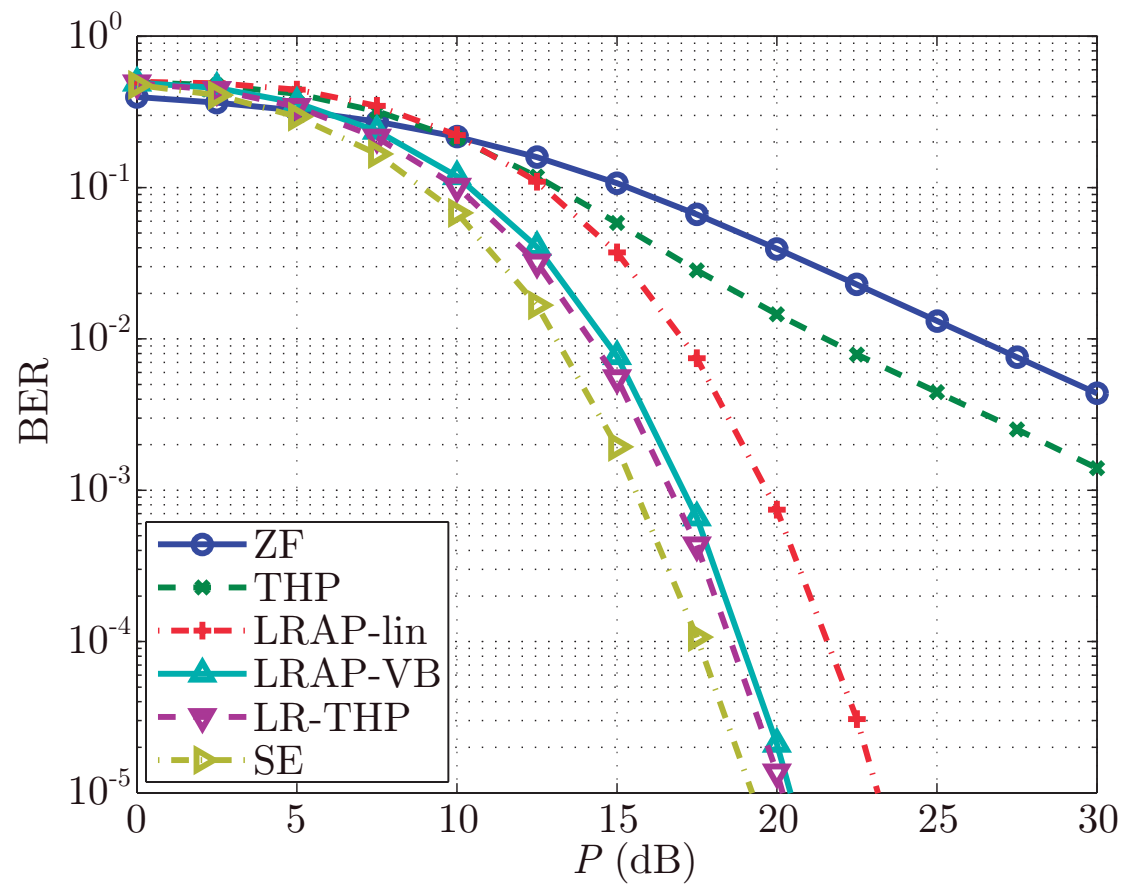

Figure 3.9: BER for the different precoding techniques for $N_{t}=M=8$ and 4-QAM.

different diversity orders achieved by the techniques cause a large difference in the performance for the high SNR regime.

The BER for $N_{t}=M=8$ is shown in Fig. 3.9. Results show that the differences between the algorithms have slightly increased: the improvement of THP compared to $\mathrm{ZF}$ is more noticeable and the performance of LRAP-VB and LR-THP is slightly further from SE. However, the performance of the LR-based techniques is still close to $\mathrm{SE}$.

Figure 3.10 shows the system sum-rate for the different techniques, which have been expressed throughout this chapter. Again, it can be seen that LRAP-VB and LR-THP achieve almost the same sum-rate as SE. It can also be noted that the power efficiency of $\mathrm{ZF}$ is far from THP, whose sum-rate is only slightly lower than the LRAP-lin one.

Similar results have been obtained for $N_{t}=M=8$ in Fig. 3.11. As for the BER case, the difference between the sum-rate of the LR-based techniques and the SE increases with the system dimension. In addition, the sum-rate achieved by THP and LRAP-lin is almost identical in this case. 


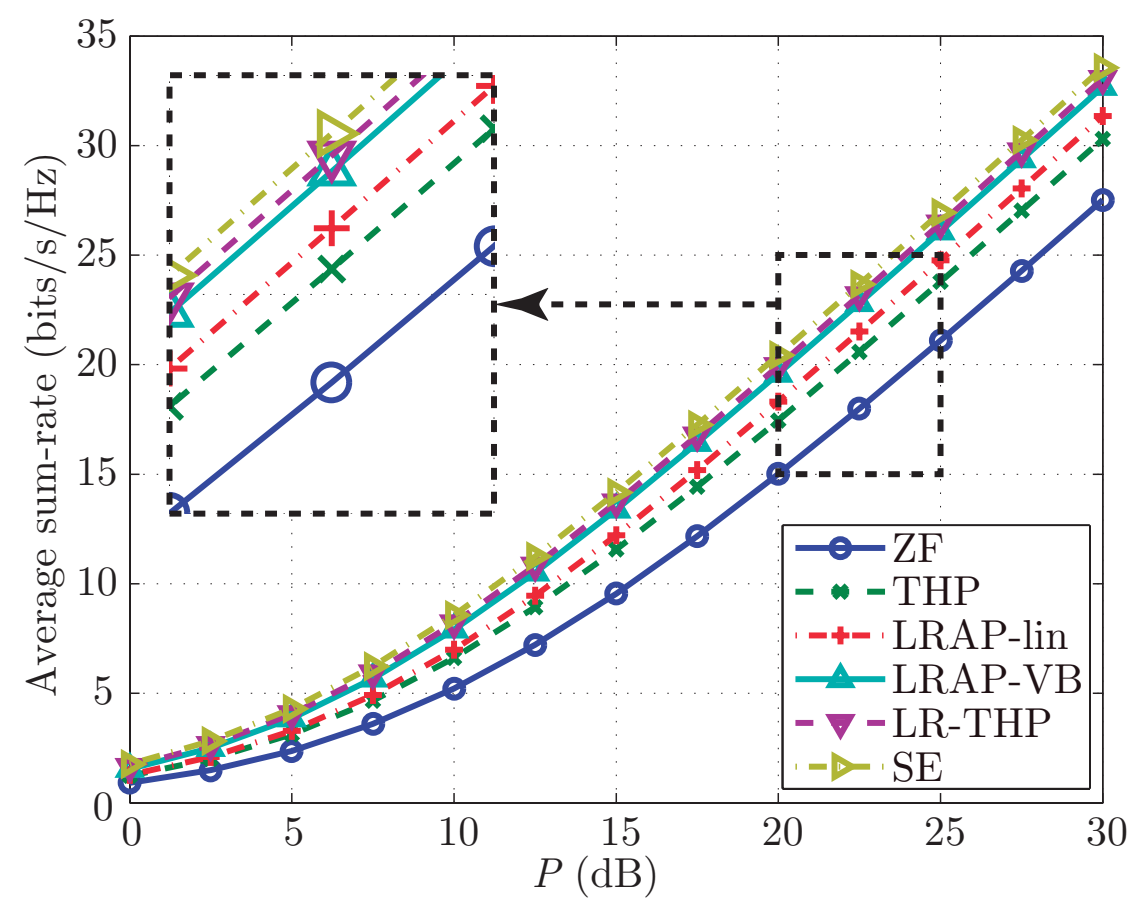

Figure 3.10: Sum-rate for the different precoding techniques for $N_{t}=M=4$.

\subsection{Complexity analysis}

In practical applications, the computational complexity restrictions can help select the most suitable algorithm to be used in practice. Thus, it is important to analyze the different precoding schemes from a computational perspective.

The main problem with SE is its high and variable complexity [200]. Its expected complexity is generally exponential and, although tractable, it is significantly more computationally demanding than the rest of algorithms presented in this chapter. In addition, its variable complexity makes it difficult to implement for real time applications. Although a fixed complexity version of this algorithm has been proposed, it suffers from a small performance degradation compared to the original algorithm [216]. A brief comparison between the SE and other LR-based techniques has been carried out in [15], concluding that the complexity of the SE considerably exceeds the computational complexity of the other algorithms. For this reason, the $\mathrm{SE}$ is excluded from this analysis.

An interesting fact is that the overall computational cost of the precoding algorithms described above stems from two different points that must be analyzed 


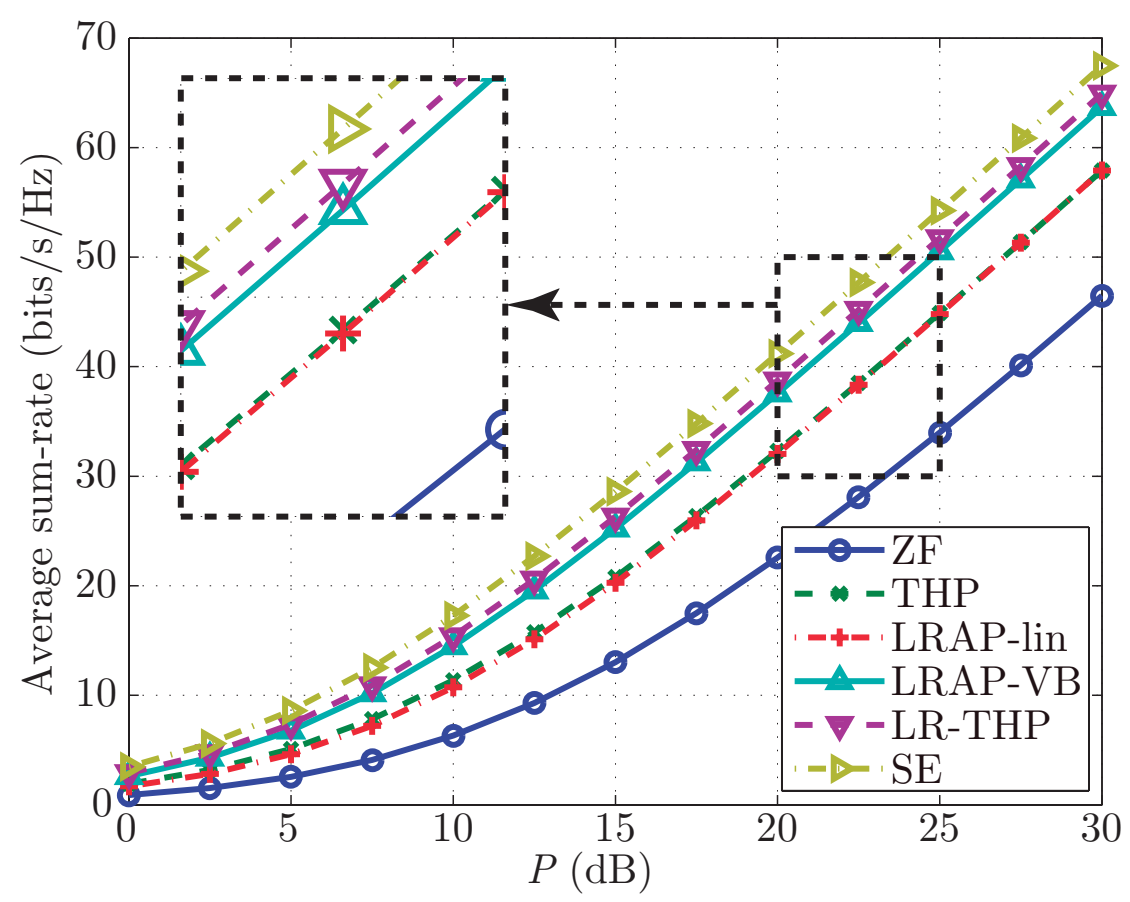

Figure 3.11: Sum-rate for the different precoding techniques for $N_{t}=M=8$.

independently. On the one hand, the preprocessing stages (matrix inversion, QR decomposition, LR, etc.) together with some matrix-matrix products that do not depend on the symbol-vector to transmit are only carried out when the channel changes and therefore, they can be performed off-line. On the other hand, the remaining calculations are carried out every time that a new symbol vector is transmitted, which happens several times for a given realization of a block-fading channel.

\subsubsection{Preprocessing computational cost}

The preprocessing stage is responsible for the calculations related to the channel, not to the signal vector. The typical operations in this stage are QR decompositions, LRs and matrix inversions. Table 3.1 shows which of the precoding algorithms under study carry out these operations:

It is important to note that the THP scheme has been considered for the ZF implementation by suppressing the modulo operation. This is due to the lower computational complexity of the QR decomposition compared to the pseudoinverse. The number of arithmetic operations of the LQ decomposition is $2(2 M)^{2}\left(2 M-2 N_{t} / 3\right)$ 
Table 3.1: Main preprocessing stages of precoding algorithms.

\begin{tabular}{c||c|c|c|c} 
& QR & LLL & $\mathbf{R}^{-1}$ & $\mathbf{H}^{\dagger}$ \\
\hline \hline ZF & Yes & No & No & No \\
\hline THP & Yes & No & No & No \\
\hline LRAP-lin & No & Yes & Yes & Yes \\
\hline LRAP-VB & Yes & Yes & Yes & Yes \\
\hline LR-THP & Yes & Yes & Yes & No \\
\hline
\end{tabular}

for a $2 M \times 2 N_{t}$ matrix, assuming that it has been computed through Householder reflections [217]. On the other hand, the inversion of a $2 M \times 2 M$ matrix through Gaussian elimination requires an amount of $M(2 M+1)$ divisions, $\left(2(2 M)^{3}+3(2 M)^{2}-10 M\right) / 6$ products and $\left(2(2 M)^{3}+3(2 M)^{2}-10 M\right) / 6$ sums.

It can also be noted that the pseudoinverse operation $\mathbf{Q}^{\dagger}$ in THP and LR-THP schemes has not been considered in Table 3.1 because it can be performed efficiently. Since the rows of $\mathbf{Q}_{0}$ in (3.20) are orthogonal unit vectors, i.e. $\mathbf{Q} \mathbf{Q}^{H}=\mathbf{I}$, the pseudoinverse of $\mathbf{Q}$ can be calculated as

$$
\mathbf{Q}^{\dagger}=\left(\mathbf{G Q}_{0}\right)^{\dagger}=\mathbf{Q}_{0}^{H} \mathbf{G}^{-1}
$$

requiring only the inversion of a diagonal matrix and the product between a matrix and a diagonal matrix.

Obtaining the total number of arithmetic operations required by the LLL algorithm is difficult due to the lack of a bounded worst-case complexity. For this reason, the fixed complexity LLL algorithm presented in [218] has been considered. In addition, the extension of the LLL algorithm to obtain the inverse of the transformation matrix, $\mathbf{R}^{-1}$, has also been considered. This extension saves some computational cost when this matrix is required. Inside each LLL loop, there are at most $(2 M-1)$ size-reductions, with $\left(4 N_{t}-1\right)$ sums and $\left(4 N_{t}-1\right)$ products each. Also, a maximum number of $8 N_{t}+8 M$ products and $4 N_{t}+4 M$ sums are needed for the QR update of a matrix with a maximum size of $2 M \times 2 N_{t}$ [192]. Note that the cost of the column swap has been disregarded, as there is no implicit arithmetic operation in it. However, the actual cost of the method can be far from the worst-case, since the LLL conditions are not always fulfilled. Thus, the average number of arithmetic operations obtained through simulations will be used instead of the worst-case.

Finally, the number of sums and products of any other preprocessing calculations apart from the ones discussed in Table 3.1 are included in Table 3.2. Basically, these calculations are needed to perform $\mathbf{Q}^{\dagger}$ as seen in (3.43) (ZF, THP, LR-THP), 
matrix products related to the pseudoinverse of the channel matrix (LRAP-lin, LRAP-VB) and some other matrix products.

Table 3.2: Additional preprocessing cost of precoding algorithms.

\begin{tabular}{c||c|c} 
& Sums & Products \\
\hline \hline ZF & - & $4 M N_{t}+2 M$ \\
\hline THP & - & $4 M N_{t}+2 M$ \\
\hline LRAP-lin & $16 M^{2} N_{t}-4 M^{2}-4 M N_{t}$ & $16 M^{2} N_{t}$ \\
\hline LRAP-VB & $24 M^{2} N_{t}-8 M^{2}-4 M N_{t}$ & $24 M^{2} N_{t}$ \\
\hline LR-THP & - & $4 M N_{t}+2 M$ \\
\hline
\end{tabular}

\subsubsection{Per-symbol-vector computational cost}

Table 3.3 collects the number of sums and products in the per-symbol-vector stage for the five precoding algorithms under study. This stage is responsible for calculating the precoded signal vector $\mathbf{x}$ from the original signal vector $\mathbf{s}$.

The per-symbol-vector arithmetic operations of the ZF and THP schemes come from equations (3.21)-(3.24) ${ }^{2}$. The results consider the modulo operation as 2 sums and 2 products according to (3.9), and take into account the power normalization by the factor $\sqrt{\gamma}$ but not its calculation. Thus, the results may be slightly different from those presented in [192], although the terms in $M^{2}$ and $M N_{t}$ match. The increased cost of the LR-THP with respect to the conventional THP is due to the product in (3.36). The operations of LRAP-lin and LRAP-VB come from the calculation of the perturbation vector $\mathbf{p}$ in (3.28) and (3.32)-(3.34), respectively, and the calculation of the precoded signal in (3.5).

Table 3.3: Per-symbol-vector cost of precoding algorithms.

\begin{tabular}{c||c|c}
\multicolumn{1}{l||}{ Sums } & Products \\
\hline \hline ZF & $2 M^{2}+4 M N_{t}-M-2 N_{t}$ & $2 M^{2}+4 M N_{t}-M+2 N_{t}$ \\
\hline THP & $2 M^{2}+4 M N_{t}+3 M-2 N_{t}$ & $2 M^{2}+4 M N_{t}+3 M+2 N_{t}$ \\
\hline LRAP-lin & $8 M^{2}+4 M N_{t}+2 M-2 N_{t}$ & $8 M^{2}+4 M N_{t}+4 M+2 N_{t}$ \\
\hline LRAP-VB & $10 M^{2}+4 M N_{t}+M-2 N_{t}$ & $10 M^{2}+4 M N_{t}+3 M+2 N_{t}$ \\
\hline LR-THP & $6 M^{2}+4 M N_{t}+M-2 N_{t}$ & $6 M^{2}+4 M N_{t}+3 M+2 N_{t}$ \\
\hline
\end{tabular}

\footnotetext{
${ }^{2}$ Note that ZF does not use the modulo operation.
} 


\subsubsection{Overall computational cost}

The overall computational cost depends on how often the preprocessing stage has to be performed, which in turn depends on the number of symbol vectors that can be transmitted through the same channel in a block fading channel or, at least, the time that CSIT remains unchanged. Hence, the overall cost of the precoding of a symbol vector is

$$
C_{\mathrm{tot}}=C_{\mathrm{psv}}+\frac{C_{\mathrm{pre}}}{L_{\mathrm{ch}}},
$$

where it has been considered that the preprocessing cost $\left(C_{\text {pre }}\right)$ is shared among $L_{\text {ch }}$ transmitted symbol vectors.

For the computational cost comparison, it is useful to know from which value of $L_{\mathrm{ch}}$ the per-symbol-vector cost $\left(C_{\mathrm{psv}}\right)$ exceeds $C_{\mathrm{pre}} / L_{\mathrm{ch}}$ and thus, the preprocessing cost decrease is not as important. Table 3.4 shows that value for the five precoding algorithms under study in a system with $N_{t}=M=4$. Note that for values of $L_{\mathrm{ch}} \leq 5$, the per-symbol-vector costs of all the algorithms are lower than their respective preprocessing costs, whereas for $L_{\mathrm{ch}}>14$ the opposite happens. It is also interesting to observe that LR-based techniques need a higher $L_{\mathrm{ch}}$ value to compensate for their more demanding preprocessing.

Table 3.4: Minimum value of $L_{\mathrm{ch}}$ for $C_{\mathrm{psv}}>\left(C_{\mathrm{pre}} / L_{\mathrm{ch}}\right)$.

\begin{tabular}{c|c|c|c|c|c} 
& ZF & THP & LRAP-lin & LRAP-VB & LR-THP \\
\hline$L_{\mathrm{ch}}$ & 5 & 5 & 9 & 14 & 10 \\
\hline
\end{tabular}

Taking into account the results above, the total number of arithmetic operations of the precoding algorithms under study for $N_{t}=4$ and different values of $M$ are shown in Fig. 3.12. For the LRAP-lin, LRAP-VB and LR-THP schemes, the computational cost with the conventional calculation of $\mathbf{R}^{-1}$ is displayed as a continuous line and the one with the extended fixed complexity LLL algorithm [219] that directly obtains $\mathbf{R}^{-1}$ is displayed as a dashed line. Figures 3.12a and $3.12 \mathrm{~b}$ show the cost for $L_{\mathrm{ch}}=5$ and $L_{\mathrm{ch}}=20$, respectively. Note that in the first case, the extended LLL algorithm slightly decreases the overall cost of the methods, whereas in the second case, the cost decrease is hardly noticeable. The reason is that for all the precoding algorithms $\left(C_{p r e} / L_{\mathrm{ch}}\right)>C_{p s v}$ when $L_{\mathrm{ch}}=5$ and therefore a decrease in the preprocessing cost is worthwhile in those cases. On the other hand, for $L_{\mathrm{ch}}=20$ the per-symbol-vector cost is predominant and masks the preprocessing cost reduction. 


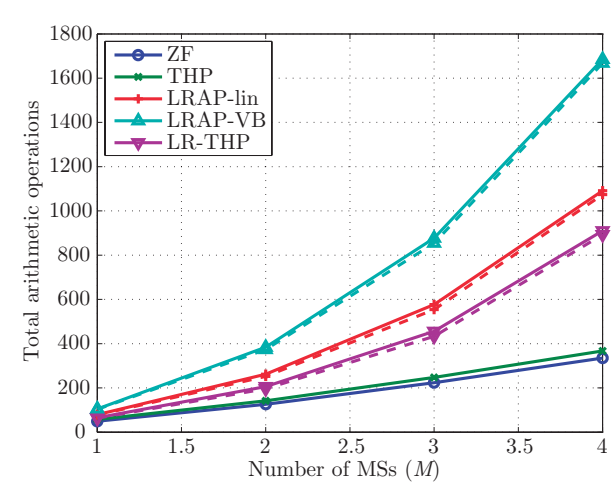

(a) $L_{\mathrm{ch}}=5$

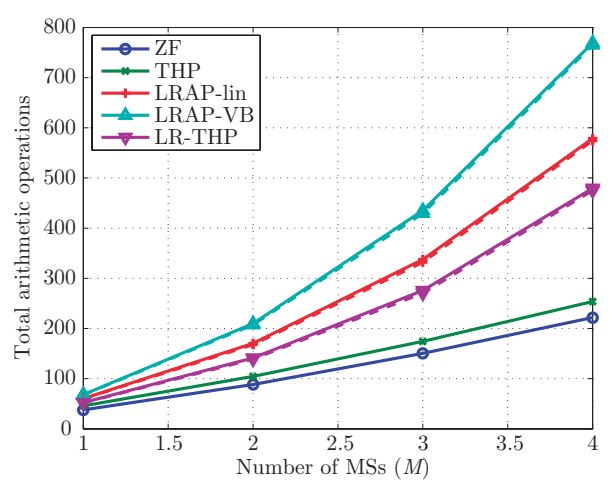

(b) $L_{\mathrm{ch}}=20$

Figure 3.12: Total number of arithmetic operations of the five precoding algorithms under study for a system with $N_{t}=4$.

\subsection{Conclusion}

This chapter has described different precoding algorithms and has presented a comparison among them in terms of BER, sum-rate and computational complexity. In Figs. 3.8-3.11 it was observed that the LR-THP and LRAP-VB methods achieved the best performance. Regarding the computational complexity, Fig. 3.12 showed that the cost of the LR-THP is significantly lower than the one of the LRAP-VB. The reason is that the LR-THP scheme is equivalent to a VP scheme where the perturbation vector has been obtained very efficiently through the modulo operation, whereas the LRAP-VB method explicitly obtains the perturbation vector and then the precoded vector. Therefore, the LR-THP method seems to be a better choice over LRAP-VB. However, in the case of tighter computational requirements, either ZF or THP should be employed instead. Whereas THP offers a better performance, ZF can be useful in some systems where linearity is important. 



\section{Chapter 4}

\section{Precoding with Imperfect CSIT}

The analysis in terms of performance and computational complexity carried out in the previous chapter has shown that ZF, THP and LR-THP are the best techniques for the various requirements. This chapter contains the analysis of the performance of these techniques when imperfect CSI is available at the transmitter [220]. Again, the performance is shown in terms of BER and sum-rate.

Chapter 3 has shown that multiuser interference can be completely suppressed through precoding at the transmitter if perfect CSIT is available. However, perfect CSIT is not possible in practical systems. Multiple errors, such as channel estimation errors, channel quantization errors, feedback errors and errors due to the channel time variation, may affect the CSIT. Thus, it is important to analyze the different precoding techniques working with imperfect CSI. Since ZF, THP and LR-THP have demonstrated to be the best precoding techniques for different performance and computational requirements, the analysis of the effect of imperfect CSIT is focused on these techniques.

Similarly to [221], the imperfect channel matrix can be modeled as

$$
\mathbf{H}_{\mathrm{e}}=\mathbf{H}+\mathbf{E},
$$

where $\mathbf{H}$ represents the actual channel matrix and $\mathbf{E}$ is an additive error matrix whose entries are i.i.d., follow a $\mathcal{C N}\left(0, \sigma_{e}^{2}\right)$ distribution and are independent of $\mathbf{H}$. Hence, the design of the precoding at the BS is obtained from $\mathbf{H}_{\mathrm{e}}$ instead of $\mathbf{H}$. 


\subsection{Zero-Forcing}

In the $\mathrm{ZF}$ scheme, the precoded signal can be expressed as

$$
\mathbf{x}=\sqrt{\gamma_{\mathrm{ZF}} \mathbf{H}_{\mathrm{e}}^{\dagger} \mathbf{s}}
$$

where $\sqrt{\gamma_{\mathrm{ZF}}}$ limits the average sum-power $\mathbb{E}\left[\|\mathbf{x}\|^{2}\right]=P$ and has been given in (3.11) for the case of perfect CSIT. In this case, it is specified as

$$
\gamma_{\mathrm{ZF}}=\frac{P / \sigma_{s}^{2}}{\operatorname{Tr}\left(\left(\mathbf{H}_{\mathrm{e}} \mathbf{H}_{\mathrm{e}}^{H}\right)^{-1}\right)}=\frac{P / \sigma_{s}^{2}}{\sum_{m=1}^{M}\left(1 / \lambda_{m}^{2}\right)},
$$

where $\lambda_{m}$ is the $m$ th singular value of $\mathbf{H}_{\mathrm{e}}$.

Now, the received signal by the MSs is expressed as

$$
\begin{aligned}
\mathbf{y} & =\mathbf{H} \mathbf{x}+\mathbf{n}=\sqrt{\gamma_{\mathrm{ZF}}} \mathbf{H H}_{\mathrm{e}}^{\dagger} \mathbf{s}+\mathbf{n}=\sqrt{\gamma_{\mathrm{ZF}}}\left(\mathbf{H}_{\mathrm{e}}-\mathbf{E}\right) \mathbf{H}_{\mathrm{e}}^{\dagger} \mathbf{s}+\mathbf{n}= \\
& =\sqrt{\gamma_{\mathrm{ZF}}} \mathbf{s}-\sqrt{\gamma_{\mathrm{ZF}}} \mathbf{E} \mathbf{H}_{\mathrm{e}}^{\dagger} \mathbf{s}+\mathbf{n},
\end{aligned}
$$

where the term $\sqrt{\gamma_{\mathrm{ZF}} \mathbf{E H}} \mathbf{e}_{\mathrm{e}}^{\dagger} \mathbf{s}$ represents the interference, which is not completely cancelled. The autocorrelation matrix of the interference-plus-noise term is given by $[220]$

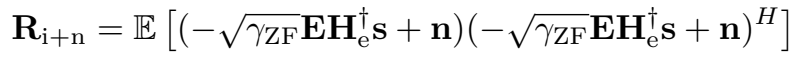

$$
\begin{aligned}
& =\gamma_{\mathrm{ZF}} \sigma_{s}^{2} \mathbb{E}\left[\mathbf{E H}_{\mathrm{e}}^{\dagger}\left(\mathbf{H}_{\mathrm{e}}^{\dagger}\right)^{H} \mathbf{E}^{H}\right]+\mathbf{I}= \\
& =\gamma_{\mathrm{ZF}} \sigma_{s}^{2} \sigma_{e}^{2} \operatorname{Tr}\left(\boldsymbol{\Sigma}^{-2}\right) \mathbf{I}+\mathbf{I}=\left(P \sigma_{e}^{2}+1\right) \mathbf{I},
\end{aligned}
$$

where the SVD $\mathbf{H}_{\mathrm{e}}=\mathbf{U} \boldsymbol{\Sigma} \mathbf{V}^{H}$ and the property that the statistics of matrix $\mathbf{E}$ do not change when multiplied by a unitary matrix such as $\mathbf{V}$ have been applied. Thus, the SINR of each user and the system sum-rate can be expressed respectively as

$$
\begin{aligned}
& \mathrm{SINR}_{\mathrm{ZF}}=\frac{\gamma_{\mathrm{ZF}} \sigma_{s}^{2}}{P \sigma_{e}^{2}+1}=\frac{1}{\left(\sigma_{e}^{2}+1 / P\right) \sum_{m=1}^{M}\left(1 / \lambda_{m}^{2}\right)} \\
& R_{\mathrm{ZF}}=M \log _{2}\left(1+\frac{1}{\left(\sigma_{e}^{2}+1 / P\right) \sum_{m=1}^{M}\left(1 / \lambda_{m}^{2}\right)}\right)
\end{aligned}
$$

It is important to point out that the term $\sigma_{e}^{2}$ in (4.7) limits the sum-rate, i.e., the system sum-rate presents an asymptote for $P \rightarrow \infty$ in

$$
R_{\mathrm{ZF}}=M \log _{2}\left(1+\frac{1}{\sigma_{e}^{2} \sum_{m=1}^{M}\left(1 / \lambda_{m}^{2}\right)}\right) .
$$

To mitigate this problem, robust versions of linear precoders have been proposed in $[222,223]$. 


\subsection{Tomlinson-Harashima precoding}

When imperfect CSIT is available in the THP scheme, the signal before the linear filtering, $\tilde{\mathbf{x}}$, and the precoded signal, $\mathbf{x}$, can be expressed as

$$
\begin{gathered}
\tilde{\mathbf{x}}=\mathbf{L}^{-1}(\mathbf{s}+\mathbf{p}), \\
\mathbf{x}=\sqrt{\gamma_{\mathrm{THP}}} \mathbf{Q}^{\dagger} \mathbf{L}^{-1}(\mathbf{s}+\mathbf{p}) .
\end{gathered}
$$

where matrix $\mathbf{L}$ is lower triangular with ones in its diagonal, $\mathbf{Q}$ contains orthogonal rows and both matrices have been obtained through an LQ decomposition of the imperfect channel matrix, $\mathbf{H}_{\mathrm{e}}=\mathbf{L Q}$. This decomposition has been detailed in (3.19)-(3.20) for the perfect CSIT case. Factor $\sqrt{\gamma_{\mathrm{THP}}}$ limits the average sumpower $\mathbb{E}\left[\|\mathbf{x}\|^{2}\right]=P$ and is given by

$$
\gamma_{\mathrm{THP}}=\frac{\mathcal{M}-1}{\mathcal{M}} \frac{P / \sigma_{s}^{2}}{\sum_{m=1}^{M}\left(1 / l_{0, m m}^{2}\right)} .
$$

The received signal can be expressed as

$$
\begin{aligned}
\mathbf{y} & =\mathbf{H} \mathbf{x}+\mathbf{n}=\left(\mathbf{H}_{\mathrm{e}}-\mathbf{E}\right) \mathbf{x}+\mathbf{n}= \\
& =\sqrt{\gamma_{\mathrm{THP}}}(\mathbf{s}+\mathbf{p})-\sqrt{\gamma_{\mathrm{THP}} \mathbf{E} \mathbf{Q}^{\dagger} \tilde{\mathbf{x}}+\mathbf{n}}
\end{aligned}
$$

Here, the interference is represented by the term $\sqrt{\gamma_{\mathrm{THP}}} \mathbf{E} \mathbf{Q}^{\dagger} \tilde{\mathbf{x}}$. Similarly, the autocorrelation matrix of the interference-plus-noise term is given by [220]

$$
\begin{aligned}
& \mathbf{R}_{\mathrm{i}+\mathrm{n}}=\mathbb{E}\left[\left(-\sqrt{\gamma_{\mathrm{THP}} \mathbf{E} \mathbf{Q}^{\dagger}} \tilde{\mathbf{x}}+\mathbf{n}\right)\left(-\sqrt{\gamma_{\mathrm{THP}}} \mathbf{E} \mathbf{Q}^{\dagger} \tilde{\mathbf{x}}+\mathbf{n}\right)^{H}\right] \\
& =\gamma_{\mathrm{THP}} \sigma_{\tilde{\mathbf{x}}}^{2} \mathbb{E}\left[\mathbf{E} \mathbf{Q}^{\dagger}\left(\mathbf{Q}^{\dagger}\right)^{H} \mathbf{E}^{H}\right]+\mathbf{I}= \\
& =\gamma_{\mathrm{THP}} \sigma_{\tilde{\mathbf{x}}}^{2} \sigma_{e}^{2} \operatorname{Tr}\left(\mathbf{G}^{-2}\right) \mathbf{I}+\mathbf{I}= \\
& =\left(P \sigma_{e}^{2}+1\right) \mathbf{I} \text {, }
\end{aligned}
$$

where matrix $\mathbf{G}$ is obtained in the LQ decomposition, as seen in (3.20). Therefore, the SINR of each user and the system sum-rate can be expressed respectively as

$$
\begin{aligned}
& \operatorname{SINR}_{\mathrm{THP}}=\frac{\gamma_{\mathrm{THP}} \sigma_{s}^{2}}{P \sigma_{e}^{2}+1}=\frac{\mathcal{M}-1}{\mathcal{M}} \frac{1}{\left(\sigma_{e}^{2}+1 / P\right) \sum_{m=1}^{M}\left(1 / l_{0, m m}^{2}\right)}, \\
& R_{\mathrm{THP}}=M \log _{2}\left(1+\frac{\mathcal{M}-1}{\mathcal{M}} \frac{1}{\left(\sigma_{e}^{2}+1 / P\right) \sum_{m=1}^{M}\left(1 / l_{0, m m}^{2}\right)}\right) .
\end{aligned}
$$


Likewise, the term $\sigma_{e}^{2}$ in (4.7) limits the sum-rate, i.e., the system sum-rate presents an asymptote for $P \rightarrow \infty$ in

$$
R_{\mathrm{THP}}=M \log _{2}\left(1+\frac{\mathcal{M}-1}{\mathcal{M}} \frac{1}{\sigma_{e}^{2} \sum_{m=1}^{M}\left(1 / l_{0, m m}^{2}\right)}\right) .
$$

Robust versions of THP that improve the performance of a system with imperfect CSIT have been proposed in [224-227]

\subsection{Lattice-Reduction-aided Tomlinson-Harashima precoding}

As seen in section 3.4.3, the THP scheme can be performed over the lattice-reduced channel if the original signal is transformed according to (3.36). In this case, the LR is performed on the imperfect channel matrix such that:

$$
\mathbf{H}_{\mathrm{e}}=\mathbf{R}_{\mathrm{e}} \widetilde{\mathbf{H}}_{\mathrm{e}}
$$

Equation (4.17) can be expressed as

$$
\widetilde{\mathbf{H}}_{\mathrm{e}}=\mathbf{R}_{\mathrm{e}}^{-1} \mathbf{H}_{\mathrm{e}}=\mathbf{R}_{\mathrm{e}}^{-1}(\mathbf{H}+\mathbf{E})=\widetilde{\mathbf{H}}+\widetilde{\mathbf{E}},
$$

where $\widetilde{\mathbf{H}}=\mathbf{R}_{\mathrm{e}}^{-1} \mathbf{H}, \widetilde{\mathbf{E}}=\mathbf{R}_{\mathrm{e}}^{-1} \mathbf{E}$ and $\mathbf{R}_{\mathrm{e}}^{-1}$ is an unimodular matrix with integer elements. Since the elements of $\mathbf{E}$ are i.i.d., the elements of $\widetilde{\mathbf{E}}$ will have an equal or higher variance than the elements of $\widetilde{\mathbf{E}}$, with equality only when there are no combinations of elements from $\mathbf{E}$, i.e., only one non-zero element in each row of $\mathbf{R}_{\mathrm{e}}^{-1}$ that must be 1 or -1 . However, this larger channel uncertainty can be better tolerated due to the higher orthogonality of $\widetilde{\mathbf{H}}$ compared to $\mathbf{H}$ [186].

As in the THP case, the SINR of each user and the system sum-rate can be expressed respectively as

$$
\begin{aligned}
& \mathrm{SINR}_{\mathrm{LR}-\mathrm{THP}}=\frac{\gamma_{\mathrm{THP}} \sigma_{s}^{2}}{P \sigma_{e}^{2}+1}=\frac{\mathcal{M}-1}{\mathcal{M}} \frac{1}{\left(\sigma_{e}^{2}+1 / P\right) \sum_{m=1}^{M}\left(1 / l_{0, m m}^{2}\right)}, \\
& R_{\mathrm{LR}-\mathrm{THP}}=M \log _{2}\left(1+\frac{\mathcal{M}-1}{\mathcal{M}} \frac{1}{\left(\sigma_{e}^{2}+1 / P\right) \sum_{m=1}^{M}\left(1 / l_{0, m m}^{2}\right)}\right),
\end{aligned}
$$

where the difference lies in the fact that $l_{0, m m}$ comes from the LQ decomposition of the lattice-reduced channel $\widetilde{\mathbf{H}}_{\mathrm{e}}$. 


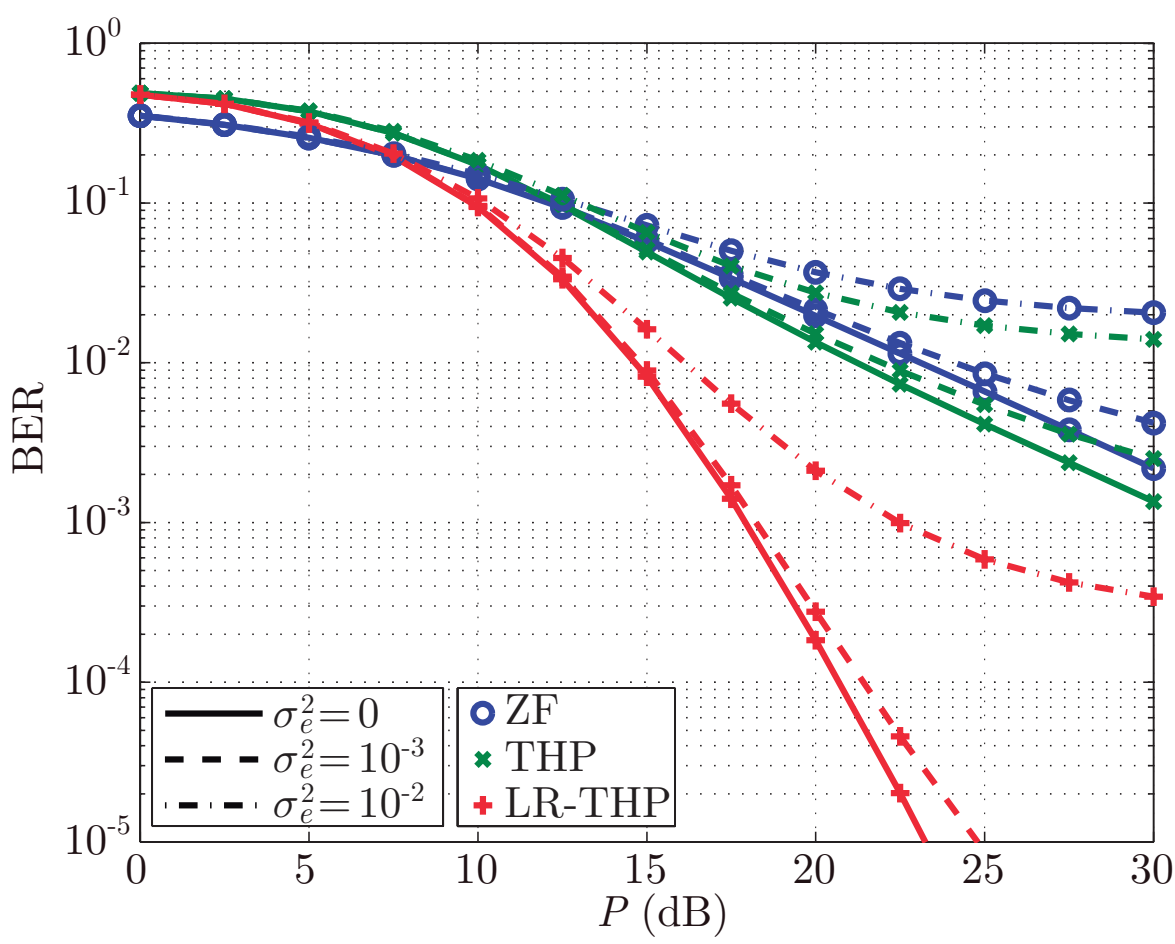

Figure 4.1: BER for ZF, THP and LR-THP, $N_{t}=M=4$, 4-QAM and $\sigma_{e}^{2}=$ $0,10^{-2}, 10^{-3}$.

\subsection{Performance}

In this section, the performance of these precoding algorithms in the case of imperfect CSIT is evaluated. Again, the performance is obtained in terms of the BER and the system sum-rate.

Figure 4.1 shows the BER for the precoding algorithms under evaluation with $N_{t}=M=4$ and different variances of the channel error, $\sigma_{e}^{2}$. In the figure, two different regions can be distinguished: a noise-limited region and an interferencelimited region. Considering the term $\sigma_{e}^{2}+1 / P$ which appears in the previous SINR expressions, the value of $P$ that produces the same noise and interference perturbation can be calculated as $P=1 / \sigma_{e}^{2}$. These values correspond to $P=30$ $\mathrm{dB}$ and $P=20 \mathrm{~dB}$ for $\sigma_{e}^{2}=10^{-3}$ and $\sigma_{e}^{2}=10^{-2}$, respectively. Hence, the region to the left of these values represents the noise-limited region and the region to the right represents the interference-limited region.

In the noise-limited region, it can be observed that there is almost no difference between the perfect and the imperfect CSIT cases. However, in the interference- 


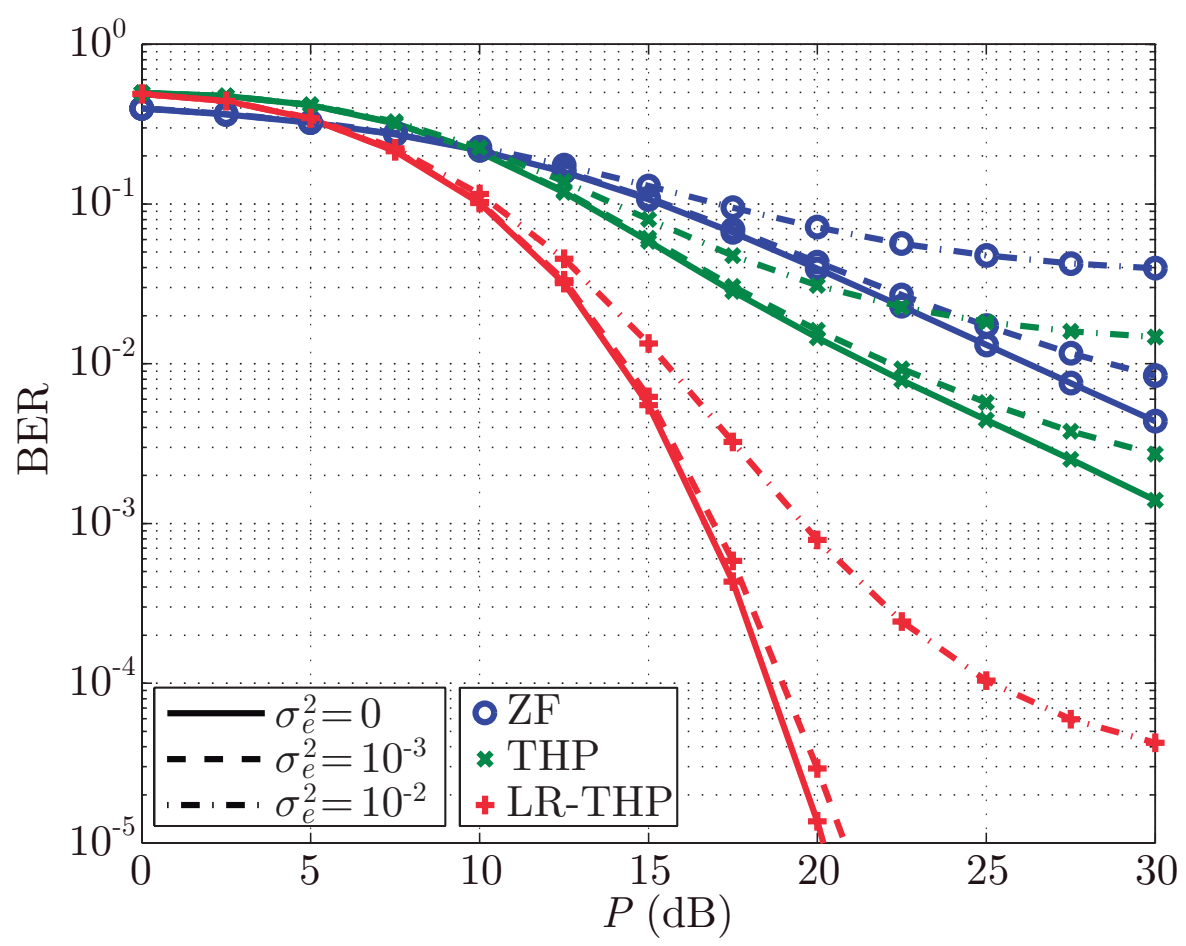

Figure 4.2: BER for ZF, THP and LR-THP, $N_{t}=M=8$, 4-QAM and $\sigma_{e}^{2}=$ $0,10^{-2}, 10^{-3}$.

limited region, the difference between the systems with different channel error increases. In this region, it can be noted that the BER does not decrease at the same rate than in the noise-limited region. In addition, the BER presents an error floor for high values of $P$. This fact can easily be appreciated in the trace corresponding to $\sigma_{e}^{2}=10^{-2}$.

Regarding the different precoding schemes, Fig. 4.1 shows that THP provides a gain of around $3 \mathrm{~dB}$ over ZF for a given BER. However, both schemes perform far from LR-THP for perfect and imperfect CSIT. Although the LR process can increase the channel uncertainty in the reduced channel as seen in (4.18), LR-THP has demonstrated to provide a better performance in the case of imperfect CSIT as well.

Figure 4.2 shows the same behavior than Fig. 4.1. These results show that the difference between ZF and THP has slightly increased. In addition, the difference between these algorithms and LR-THP have also increased for a high $P$ due to the higher diversity order of the LR-based techniques. 


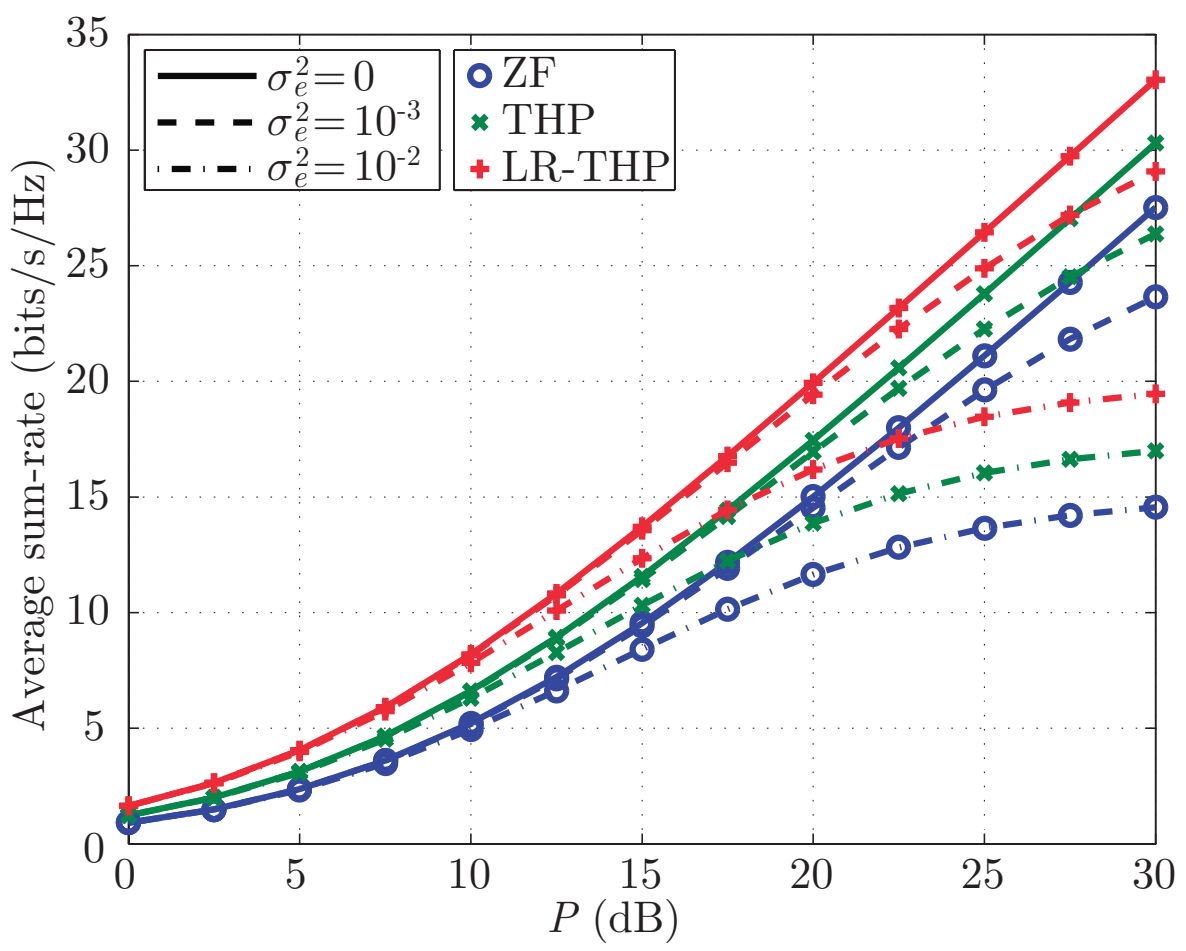

Figure 4.3: Sum-rate for ZF, THP and LR-THP, $N_{t}=M=4$ and $\sigma_{e}^{2}=0,10^{-2}, 10^{-3}$.

The average sum-rate for a system with $N_{t}=M=4$ and different variances of the channel error, $\sigma_{e}^{2}$, is shown in Fig. 4.3. In this figure, the difference between the noise-limited and the interference-limited regions can also be appreciated. Whereas the difference among the systems with different $\sigma_{e}^{2}$ is insignificant in the noiselimited region, this difference is considerable in the interference-limited region. In addition, the sum-rate asymptotes due to the interference can also be noted, especially for $\sigma_{e}^{2}=10^{-2}$.

Finally, Fig. 4.4 shows the average sum-rate for a system with $N_{t}=M=8$. These results are in line with the ones in Fig. 4.3. However, it is important to highlight that channel imperfections are more noticeable in larger systems, since the aggregated interference is higher. For instance, in this case, the sum-rate achieved by $\mathrm{ZF}$ and $\sigma_{e}^{2}=10^{-2}$ is less than half of the one achieved with perfect CSIT. 


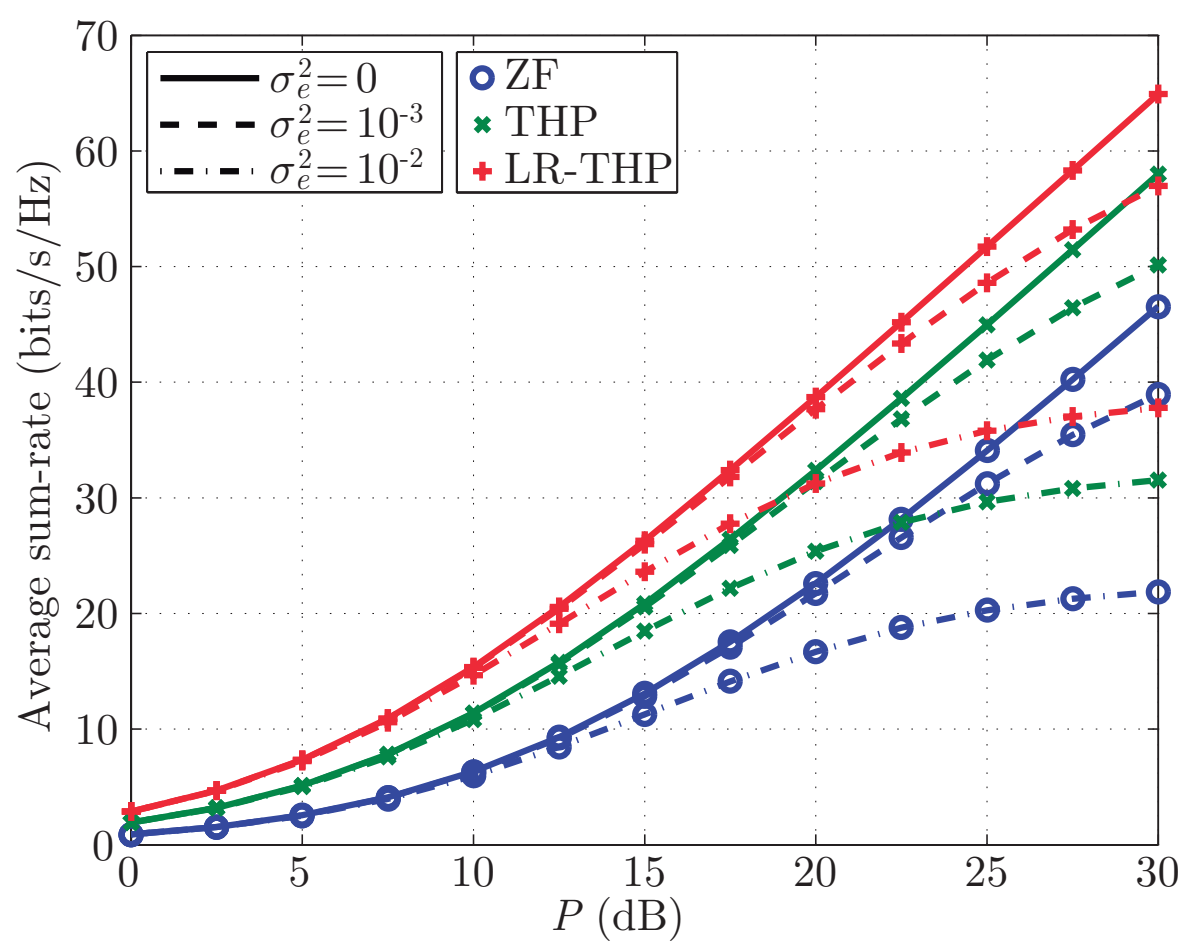

Figure 4.4: Sum-rate for ZF, THP and LR-THP, $N_{t}=M=8$ and $\sigma_{e}^{2}=0,10^{-2}, 10^{-3}$.

\subsection{Conclusion}

This chapter has presented the performance of the most interesting precoding techniques seen in chapter 3 when imperfect CSIT is available. The analysis showed that, regarding the transmit power, two different regions can be distinguished: a noise-limited region and an interference-limited region. In the noise-limited region, the channel errors are almost negligible and the results are similar to the perfect CSIT case. However, in the interference-limited region the channel errors entail a strong interference that limits the performance of the precoding algorithms. In both regions, LR-THP was the best technique in terms of performance, showing that LR is also a key technique when imperfect CSIT is available. 


\section{Chapter 5}

\section{Hardware Implementation}

In this chapter, some implementations of the precoding algorithms analyzed in Chapter 3 are presented. First, a brief introduction to GPUs as general-purpose computing devices is provided. Next, all the precoding algorithms are implemented on a GPU for a MU-MISOOFDM system [228]. Subsequently, the implementations of the THP and LR-THP algorithms are optimized and a reconfigurable THP scheme is presented [229]. Finally, the problem of LR is itself optimized since it has shown to be the main bottleneck for GPU implementations and real time applications [230-232].

In Chapter 3 an analysis of the computational complexity of the different precoding algorithms has been carried out. Although that was an interesting result, evaluating their implementations is more useful for practical systems. In this chapter, GPU implementations of the precoding algorithms are proposed and compared to their CPU counterpart.

The implementations of the precoding algorithms are evaluated in a MU-MISOOFDM system. MIMO-OFDM techniques allow transmitting different streams over the different subcarriers and, through MIMO precoding, different spatial beams in each of the subcarriers. However, this scheme requires a huge computational complexity because it is necessary to perform a precoding algorithm over all the subcarriers.

In the last few years, the use of many-core processors such as general-purpose graphic processing units (GPGPUs) is becoming attractive for the efficient implementation of algorithms in many fields of engineering [18, 233]. This is due to the cost-effectiveness of GPUs together with their tremendous capability for parallel processing. 
The chapter is organized as follows. Section 5.1 presents an introduction to the GPU architecture and its possibilities. Section 5.2 presents a GPU implementation of all the precoding techniques analyzed in Section 3.6, showing that the THP structure is the one that best adapts to the GPU architecture. Section 5.3 optimizes the THP implementation and presents a reconfigurable scheme that allows to transmit using two precoding techniques: THP and LR-THP. Finally, the problem of LR is analyzed and an efficient solution for GPU and GPU+CPU architectures is proposed, since it has shown to be the main bottleneck in the implementation of the related precoding techniques.

\subsection{Introduction to GPU and CUDA}

Traditionally, the main role of GPU has been rendering 3D scenes into a $2 \mathrm{D}$ screen. This work basically consists on continuously calculating the value of each pixel according to some complex rendering operations. Since the calculations for the different pixels can be performed independently, this task shows an enormous inherent parallelism [19, 234].

In the last few years, the demand for more powerful GPUs has increased due to the appearance of higher definition screens with higher refresh rates. In addition, the adoption of these devices as general-purpose computing resources has allowed a faster evolution. Nowadays, GPUs ${ }^{1}$ are architectures that contain hundreds of computational cores with very fast on-chip memory and high-bandwidth external memory. Due to their ubiquitous presence in computers, laptops and smartphones together with the fact that not many applications make an intense use of them, a GPU can be considered as a powerful coprocessor that allows offloading the CPU [235].

In order to understand the features of GPUs as a computational architecture, it is important to revise the two main branches microprocessor design: multicore architectures and many-core architectures [236]. A multi-core architecture (typically CPUs) may represent up to 10-12 processing cores, each designed to maximize the execution speed of sequential applications. Hence, a refined control logic and large cache memories are provided to reduce the latency. On the other hand, a many-core architecture (typically GPUs) may have hundreds of processing cores and its design focuses on the maximization of the execution throughput of parallel applications. In order to save chip real state to accommodate the large number of processing cores, pipelined memories and arithmetic operations with long latency are employed. As a result, many-core architectures may achieve 10 times as many floating-point operations per second (FLOPS) as those achieved by multicores [237]. However, these results are not directly related to execution times of applications, but are the raw speed that the computational resources can support.

\footnotetext{
${ }^{1}$ In what follows, the term GPU will refer refer to both dedicated GPUs or GPGPUs.
} 
Generally speaking, multi-core architectures will perform better in applications with a few threads, while many-core architectures will achieve better performance in applications with a large number of threads [236].

\subsubsection{CUDA}

Although GPUs prior to 2006 had powerful architectures, its use was very restricted to graphics processing. The main reason was that performing non-graphic computations using $\mathrm{Cg}$ language programming or graphics application programming interface (API) such as OpenGL was a difficult task [19, 234]. In 2006, Nvidia introduced compute unified device architecture (CUDA), a general purpose parallel computing platform and programming model that exposes the massive computation potential offered by the programmable GPU [237]. CUDA allows using a well-known programming language, such as $\mathrm{C}, \mathrm{C}++$ or Fortran, as a high level programming language in a multithreaded environment, providing instructions to support communication and synchronization.

According to the CUDA programming model, the CUDA threads are executed in a separate device operating as a coprocessor of the host. Normally, the host consists of a CPU and its corresponding random access memory (RAM) that execute one or more sequential threads. The device consists of one or several GPUs and GPU memory executing one or more parallel kernels. There are two different types of GPU memory: shared memory and global memory. The shared memory is a fast on-chip memory whose size is about several hundred of kilobytes, while the global memory is a larger and slower off-chip memory.

The kernel consists of a set of common sequential operations performed independently in all the threads. Since all the threads execute the same code, a CUDA device can be considered as a single program, multiple data (SPMD) machine [238]. This concept is similar to single-instruction multiple-data (SIMD) [239]. However, in an SIMD system, all the processing units execute the same instruction at the same time, while in an SPMD system the execution does not need to be concurrent.

Threads are organized into thread-blocks (TBs) and TBs are organized into a grid, setting up a two-level hierarchy (see Fig. 5.1). CUDA supports 1, 2 or 3dimensional TBs, as well as 1, 2 or 3-dimensional grids. All the threads in a TB have the same block index but a different thread index. Hence, a unique identifier can be generated from these indices. The number of elements in each dimension must be specified on each kernel launch [237]. Threads within a TB can synchronize through a barrier to coordinate memory accesses. In contrast, TBs are completely independent and can only share data through the global memory, as seen in Fig. 5.2. Thread organization is very important because different organizations may result in a very different performance. 


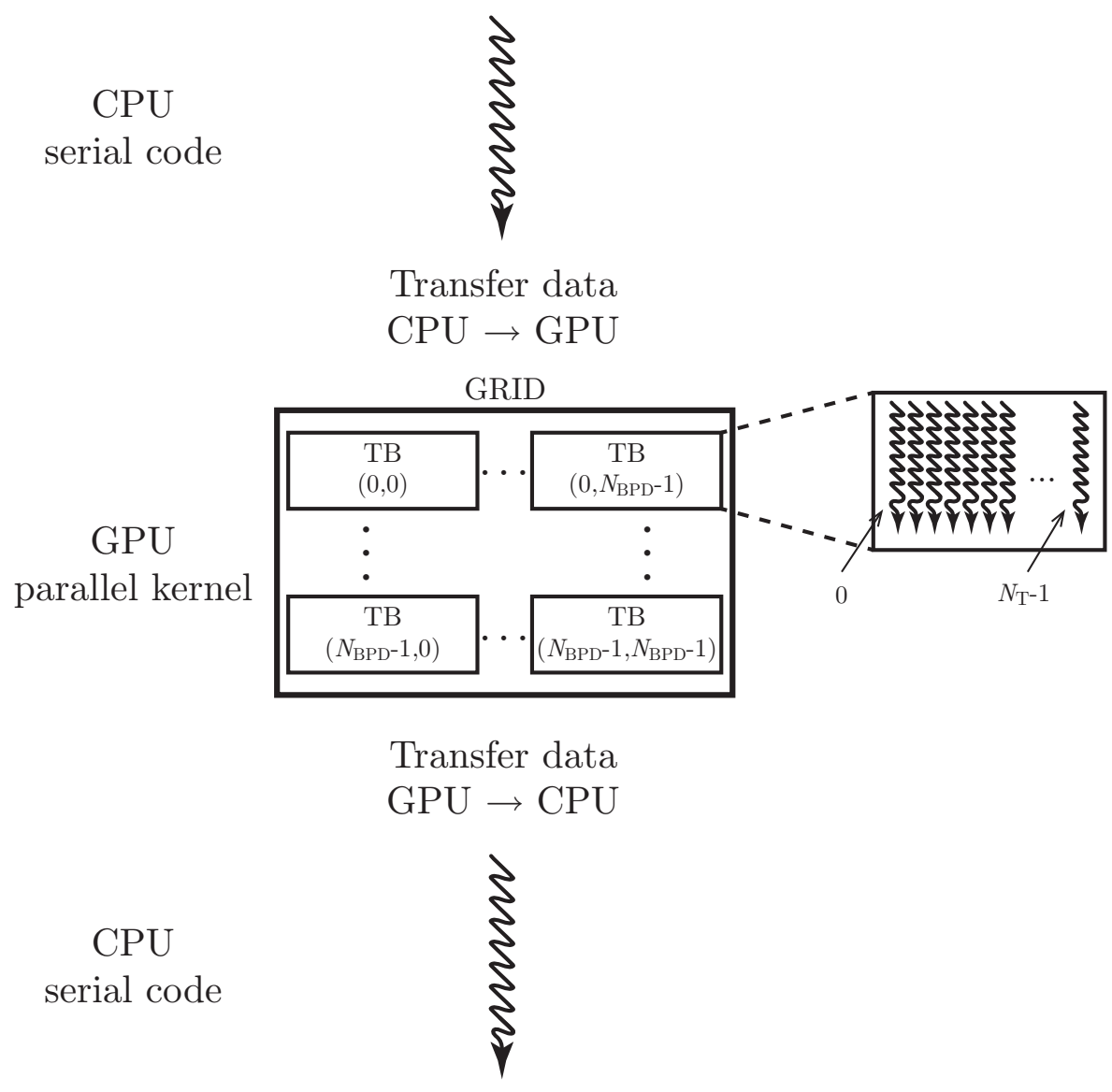

Figure 5.1: Example of execution of a CUDA program using a 2-dimensional grid with $N_{\mathrm{BPD}}$ blocks per dimension and 1-dimensional block with $N_{\mathrm{T}}$ threads.

Before performing the operations of the kernel, the global memory on the device has to be allocated and the data has to be transferred from the host memory to the device memory (see Fig. 5.2). Once the kernel execution has finished, the result data is copied from the device memory to the host memory and, if the data is no longer needed, the device memory is freed up.

Figure 5.1 shows the execution of a CUDA program. It is important to point out that the operations in the kernel are executed by a large number of threads. Once all the threads have completed their operations, the kernel finishes and the execution continues on the host. In this simplified example, the kernel is called from the CPU and there is no overlap between CPU and GPU operations. However, more advanced applications could use these features to achieve a higher performance. 


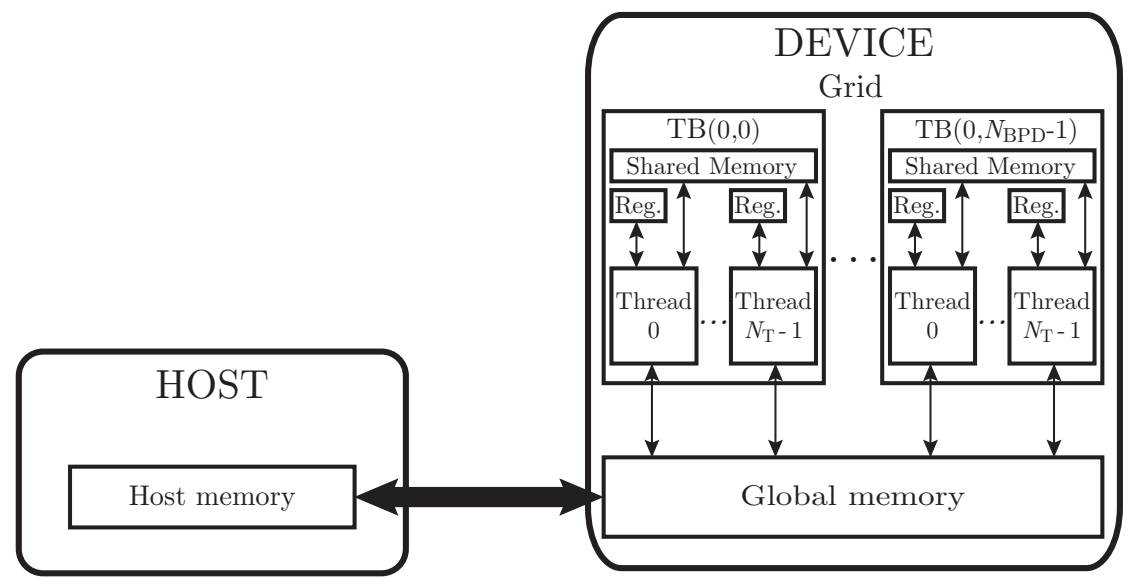

Figure 5.2: Host memory and device memory.

\subsubsection{Hardware considerations}

The CUDA GPU hardware architecture is based on an array of multithreaded streaming multiprocessors (SMs). Once a kernel is called, the blocks of the grid are distributed among the multiple SMs. The threads within a TB are executed concurrently on one SM and, depending on the TB dimension, multiple TBs can be executed concurrently. As the execution of TBs finishes, new TB are processed on the vacant SMs.

An SM basically consists of several CUDA cores for arithmetic operations, several special functions for single-precision floating-point operations and several warp schedulers. A warp is a group of 32 consecutive threads that form the basic unit of thread scheduling in SMs and its size is implementation-specific. The number of CUDA cores, special functions and warp schedulers depends on the compute capability. In the case of CUDA cores, this number ranges between 8 (compute capability 1.x) and 128 (compute capability 5.0) [237].

When one or several TBs are processed, the SM divides them into warps and the warp scheduler sets the scheduling of each one. The execution units are designed to execute all the threads in a warp following the SIMD model, i.e., each instruction is performed in all the threads in the warp. Thus, full efficiency is achieved when all the threads have the same execution path. Warp divergence occurs when threads do not follow the same execution path and it is mainly due to conditional statements or conditional loops. In this case, the SIMD model turns into a singleinstruction multiple-thread (SIMT) model where all the branches of the execution path are executed sequentially, disabling in each case the threads that are not on that path [237]. A considerable improvement can be obtained by avoiding warp divergence. 


\subsection{GPU implementation of precoding algorithms}

This section collects the results presented in [228] where a MU-MISO-OFDM system is contemplated. Considering the extension of (3.1) to the OFDM case analyzed in section 2.4 and aggregating the received signal of the different MSs in a vector, the received signal can be expressed as

$$
\mathbf{y}[k]=\mathbf{H}[k] \mathbf{x}[k]+\mathbf{n}[k] .
$$

Matrices $\mathbf{H}[k]$ used for the evaluation of the implementations follow the Gaussian i.i.d. model depicted in section 2.6.1 and no correlation between channel matrices for different subcarriers has been considered.

Tables 5.1 and 5.2 collect the steps of the different algorithms analyzed in chapter 3. It is important to point out that, as in section 3.6, the implementation of the ZF algorithm consists of using the THP scheme suppressing the modulo operation. In addition, the implementations make use of the real-valued form expressed in (3.2).

\subsubsection{Implementation details}

The implementation of multiuser precoding schemes in a MU-MISO-OFDM system is a quite expensive computational task, since the precoding algorithms must be performed for every subcarrier. In order to take advantage of the inherent parallelism of this multi-carrier system, the Nvidia Quadro FX 5800 GPU has been used for the implementations. Its specifications are given in Table 5.3.

In the proposed implementations, the operations involved in the preprocessing and the per-symbol-vector stages have been parallelized independently, defining for each stage a kernel with a different grid configuration. The first kernel includes the operations required by each one of the $K$ subcarriers during the preprocessing stage, such as QR-decompositions, lattice basis reduction and matrix inversions. These operations are performed in $R=K$ threads, that is, the operations related to different subcarriers are executed concurrently by different threads.

The second kernel is devoted to performing the various operations to obtain the precoded signal using the results obtained in the preprocessing stage, which are valid while the channel remains constant. As the channel remains constant during $L_{\text {ch }}$ symbol periods, a total of $R=K L_{\text {ch }}$ threads use the preprocessing results to obtain their precoded symbol vectors. A one-dimensional grid configuration with $N_{\mathrm{T}}=128$ number of threads per block is considered in both kernels. The number of blocks can be straightforwardly calculated as $N_{\mathrm{B}}=\left\lceil R / N_{\mathrm{T}}\right\rceil$.

Due to the large amount of required memory, the implementations only employ GPU global memory to store the data structures needed for the precoding process, 
Table 5.1: ZF and THP algorithms.

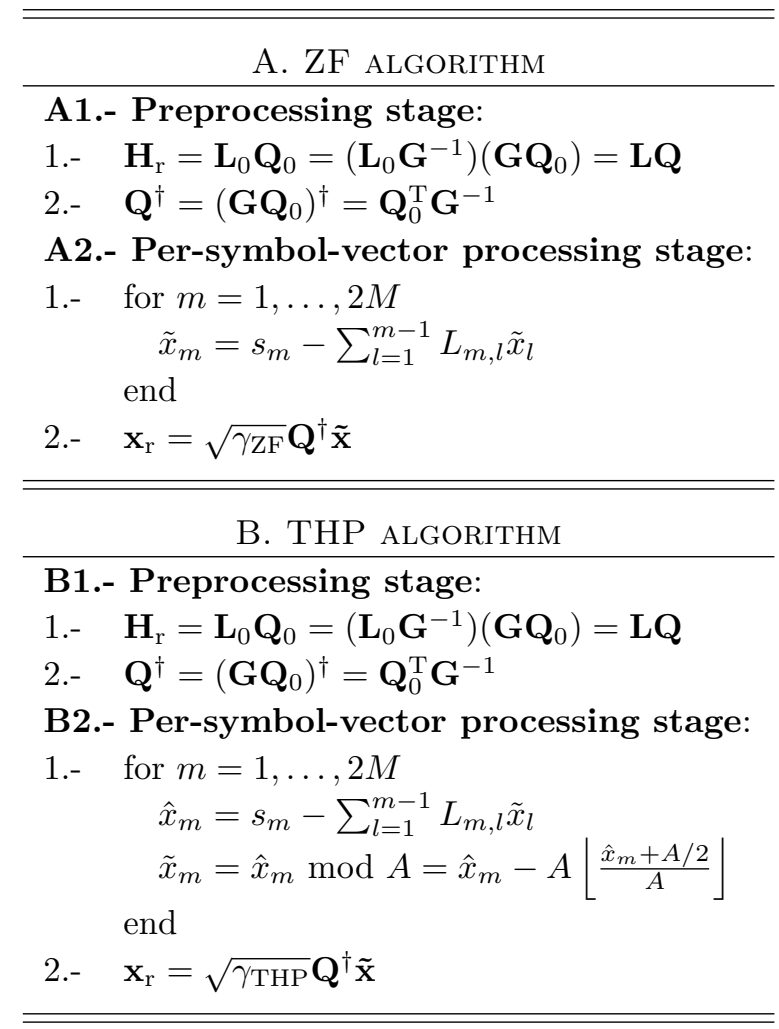

using double precision. Before starting to execute the first kernel (preprocessing stage), the channel matrices of all the subcarriers, $\mathbf{H}[k]$, are copied to the GPU global memory. When the kernel finishes, the threads store their results in the global memory of the GPU as well. These results are subsequently used by the second kernel (per-symbol-vector stage).

To carry out the next stage, where the final precoded signal is obtained, only the information symbols of all the subcarriers and MSs, $\mathbf{s}[k]$, need to be copied to the global memory, since the preprocessing results are already on the device. Once the final precoded signals are obtained by the threads, they are transferred to the CPU memory in order to be transmitted. 
Table 5.2: LRAP algorithms.

\begin{tabular}{|c|c|}
\hline \multicolumn{2}{|c|}{ C. LRAP-LIN ALGORITHM } \\
\hline \multicolumn{2}{|c|}{ C1.- Preprocessing stage: } \\
\hline \multicolumn{2}{|l|}{ 1.- $\quad \mathbf{H}_{\mathrm{r}}^{\dagger}=\widetilde{\mathbf{H}_{\mathrm{r}}^{\dagger}} \mathbf{R}$} \\
\hline \multicolumn{2}{|c|}{ C2.- Per-symbol-vector processing stage: } \\
\hline \multicolumn{2}{|c|}{ 1.- $\quad \mathbf{p}_{\mathrm{r}}=-A \mathbf{R}^{-1}\left\lceil\frac{\mathbf{R s}_{\mathrm{r}}}{A}\right\rfloor$} \\
\hline \multicolumn{2}{|c|}{ 2.- $\quad \mathbf{x}_{\mathrm{r}}=\sqrt{\gamma_{\mathrm{LRAP}-\operatorname{lin}}} \mathbf{H}_{\mathrm{r}}^{\dagger}\left(\mathbf{s}_{\mathrm{r}}+\mathbf{p}_{\mathrm{r}}\right)$} \\
\hline \multicolumn{2}{|c|}{ D. LRAP-VB ALGORITHM } \\
\hline \multicolumn{2}{|c|}{ D1.- Preprocessing stage: } \\
\hline \multicolumn{2}{|l|}{ 1.- $\quad \mathbf{H}_{\mathrm{r}}^{\dagger}=\widetilde{\mathbf{H}_{\mathrm{r}}^{\dagger}} \mathbf{R}$} \\
\hline \multicolumn{2}{|c|}{ 2.- $\quad \widetilde{\mathbf{Q H}} \tilde{r}_{\mathrm{r}}^{\dagger}=\mathbf{L}$} \\
\hline \multicolumn{2}{|c|}{ D2.- Per-symbol-vector processing stage: } \\
\hline \multicolumn{2}{|c|}{ 1.- $\quad \mathbf{q}=-\mathbf{Q} \mathbf{H}_{\mathrm{r}}^{\dagger} \mathbf{s}_{\mathrm{r}}$} \\
\hline \multicolumn{2}{|l|}{ 2.- $\quad$ for $m=1, \ldots, 2 M$} \\
\hline$\widetilde{q}_{m}=A\left\lceil\frac{q_{m}-\sum_{l=1}^{m-1} L_{m, l} \widetilde{q}_{l}}{A}\right.$ & \\
\hline end & \\
\hline 3.- $\quad \mathbf{p}_{\mathrm{r}}=\mathbf{R}^{-1} \widetilde{\mathbf{q}}$ & \\
\hline 4.- $\quad \mathbf{x}_{\mathrm{r}}=\sqrt{\gamma_{\mathrm{LRAP}-\mathrm{VB}}} \mathbf{H}_{\mathrm{r}}^{\dagger}\left(\mathbf{s}_{\mathrm{r}}+\mathbf{p}_{\mathrm{r}}\right)$ & \\
\hline
\end{tabular}

E. LR-THP ALGORITHM

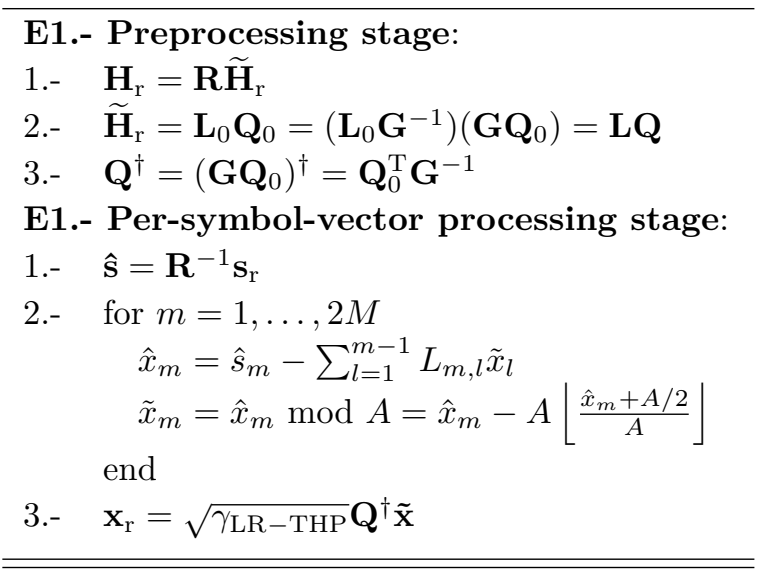


Table 5.3: Nvidia Quadro FX 5800 GP features.

\begin{tabular}{|l|l|}
\hline Compute capability & 1.3 \\
\hline Number of SM & 30 \\
\hline Number of CUDA cores & 240 \\
\hline Clock rate & $1.30 \mathrm{GHz}$ \\
\hline Global memory & $4 \mathrm{~GB}$ \\
\hline Constant memory & $64 \mathrm{kB}$ \\
\hline Shared memory per block & $16 \mathrm{kB}$ \\
\hline
\end{tabular}

\subsubsection{Results}

In this section, the execution times of the GPU and CPU implementations of the previous algorithms are evaluated. The selected CPU is an Intel Xeon E5430 at $2.66 \mathrm{GHz}$ with $32 \mathrm{~GB}$ of RAM memory and $12 \mathrm{MB}$ of cache memory. The compiler used is the Intel $\mathrm{C}$ compiler with the global performance optimization -o3.

Without loss of generality, the results consider the same number of transmit antennas and MSs, $N_{t}=M=4, L_{\mathrm{ch}}=20$ and different numbers of subcarriers, $K$. In LTE Rel. 10, the transmission bandwidth can be further expanded from the one in Rel. 8 by means of carrier aggregation, supporting transmission bandwidths up to $100 \mathrm{MHz}$ using 6000 subcarriers [240].

Figure 5.3 shows the speed-up obtained when running algorithms on the GPU compared to the CPU implementation. Note that not all the algorithms under study achieve the same speed-up, indicating that some of them are more suitable for GPU implementation than others. Algorithms such as ZF, THP or LR-THP achieve a high speed-up even for a small number of subcarriers.

In Fig. 5.4, the times of the preprocessing stage and the per-symbol-vector stage are considered independently for the speed-up calculation. Figures 5.4a and 5.4b represent the speed-up for the preprocessing stage and the per-symbol-vector stage, respectively, for a system with $N_{t}=M=4$. It can be noticed that there is not a large difference in the algorithms regarding the speed-up at the preprocessing stage. However, Fig. 5.4b shows that a parallel implementation on a GPU of the per-symbol-vector stage for ZF, THP and LR-THP can be much more efficient than its implementation on a CPU.

A comparison among the computational complexity of the precoding algorithms based on the system size was carried out in section 3.6 and in [192]. In order to validate this analysis, a comparison among their computational times has been performed for $N_{t}=M=4$ and different number of subcarriers, $K$ (Fig. 5.5). Again, it is interesting to point out that THP has almost the same computational time than $\mathrm{ZF}$, providing a better performance for a very similar computational time, 


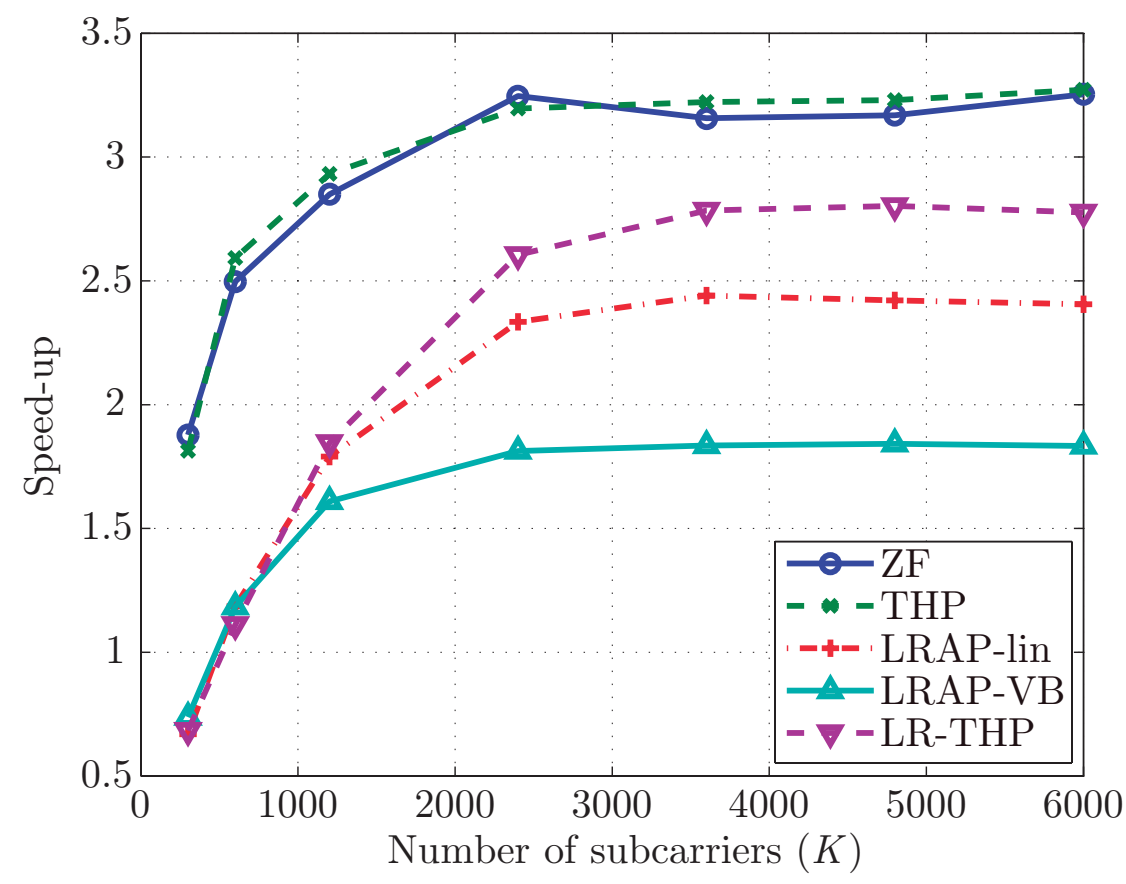

Figure 5.3: Speed-up for $N_{t}=M=4$ and different number of subcarriers, $K$.

but at the cost of non-linearity. It is also interesting to observe that, although Fig. 3.12 shows that LR-THP has a slightly lower computational complexity than LRAP-lin, its computational time is slightly higher. This difference can be justified by the fact that matrix $\mathbf{R}$ and $\mathbf{R}^{-1}$ in $\mathrm{C} 2.1$ (Table 5.2) may contain many zeros. Hence, the computational cost of these matrix products decreases and it has not been considered in section 3.6. In addition, LR-THP requires more memory accesses than LRAP-lin. However, the computational time for LR-THP is still much lower than for LRAP-VB, allowing better BER and sum-rate performances at a lower computational cost and time.

The algorithms with the best speed-ups are those that make use of the THP structure (ZF, THP, LR-THP). This fact confirms that LR-THP is the best choice to achieve quasi-optimal bit-error-rate through an efficient implementation on a GPU. 


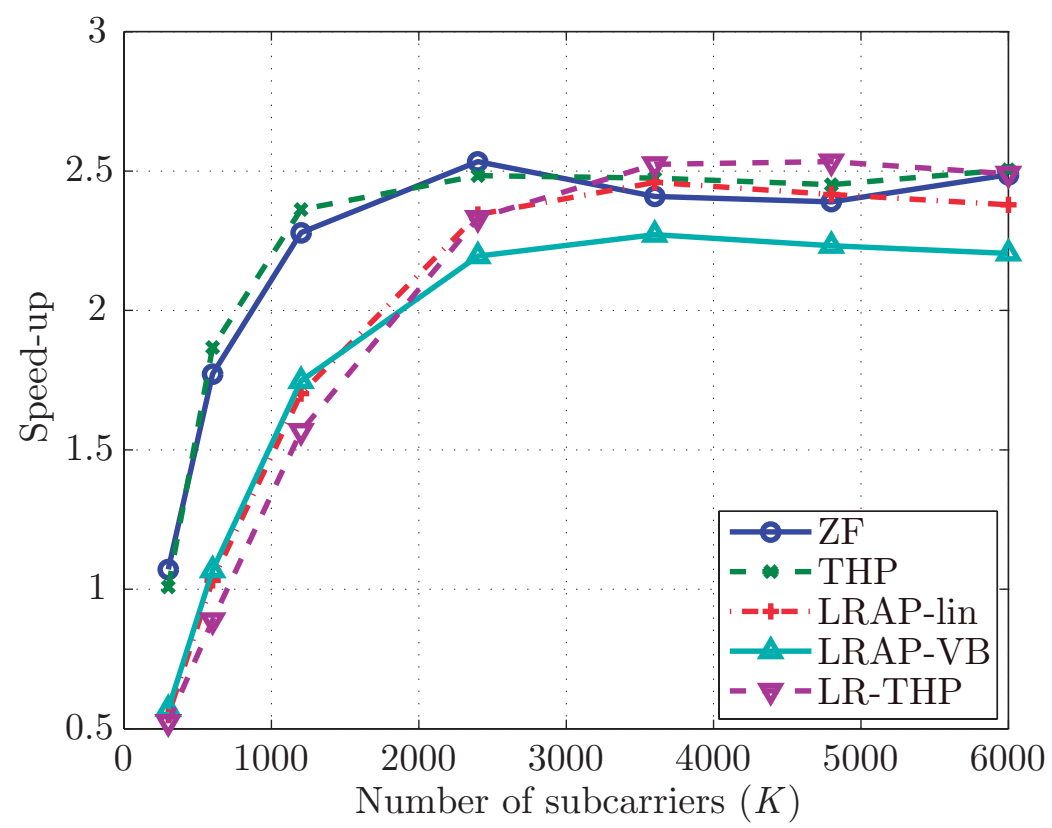

(a) Preprocessing stage

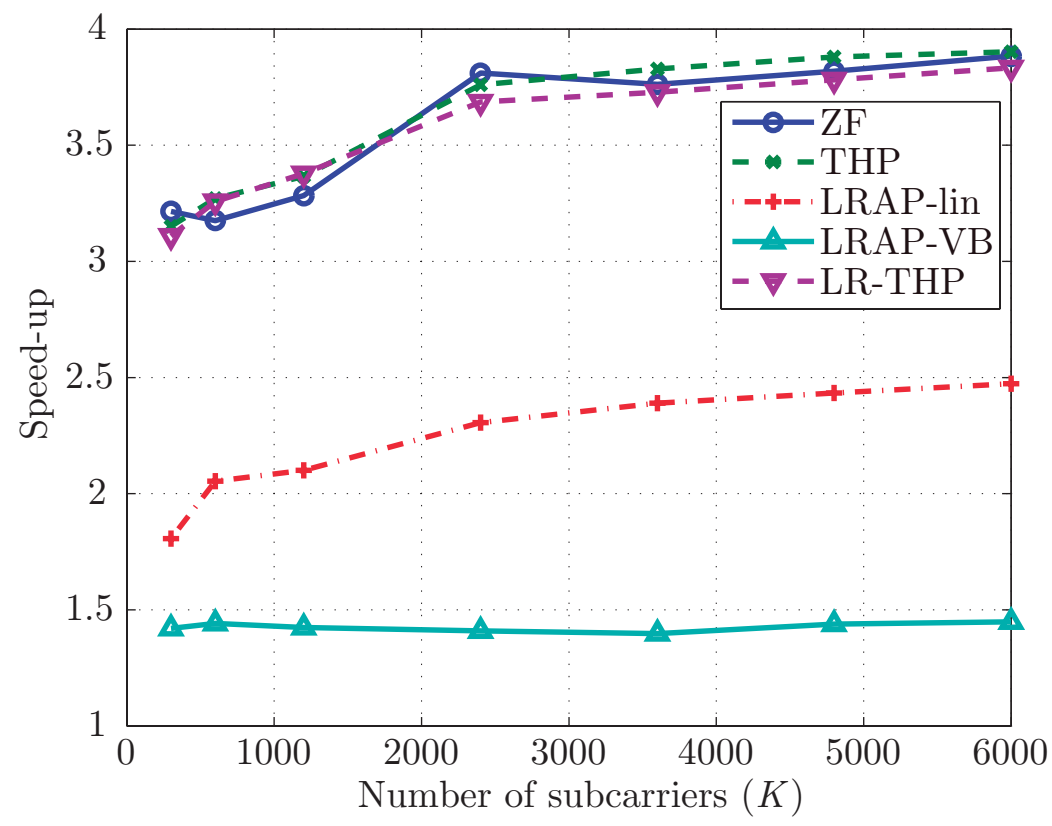

(b) Per-symbol-vector stage

Figure 5.4: Speed-up per stage for $N_{t}=M=4$ and different number of subcarriers. 


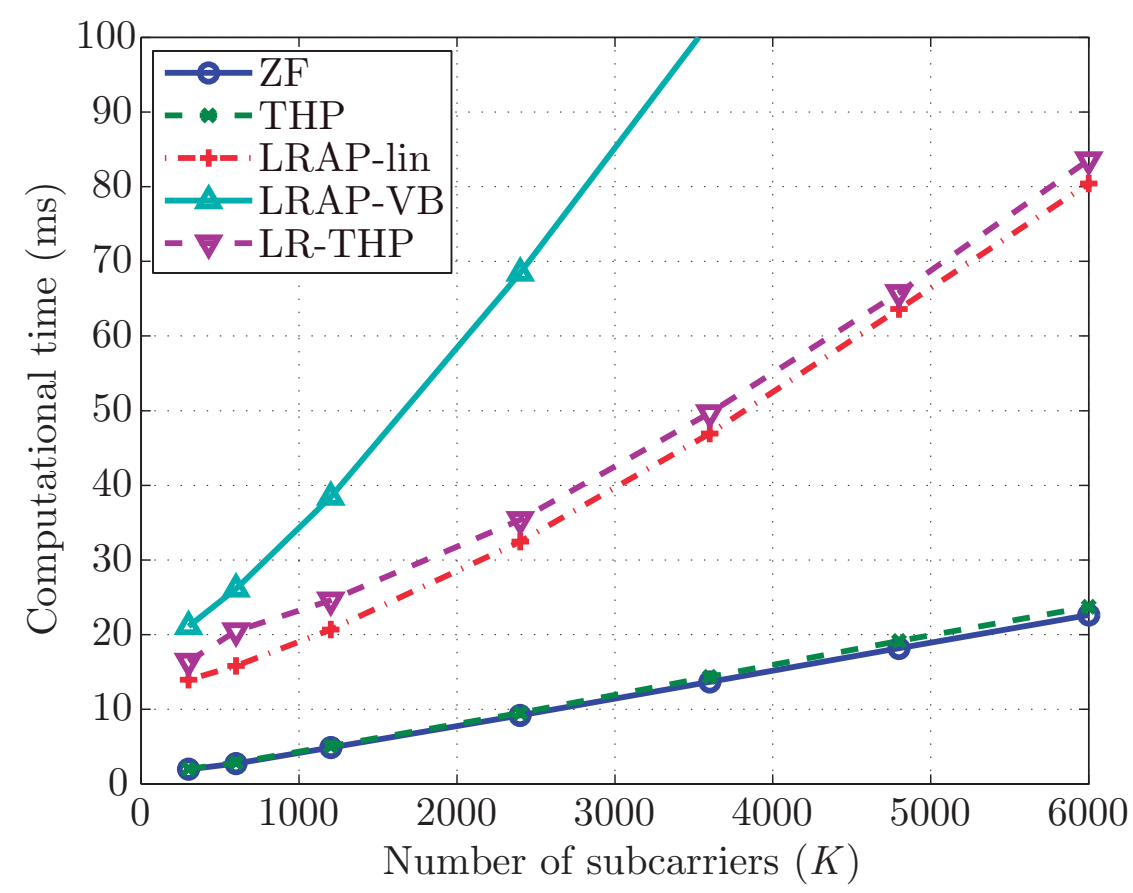

Figure 5.5: Computational time in GPU for $N_{t}=M=4$ and different number of subcarriers, $K$.

\subsection{Reconfigurable GPU implementation of THP}

Results in the previous section have shown that ZF, THP and LR-THP techniques best fit the GPU architecture. This interesting result motivated the improvement of the THP and LR-THP implementations along with a reconfigurable GPU implementation combining the use of LR [229].

In section 3.6, LR-THP demonstrated to improve the performance of conventional THP at the expense of an increased computational cost, mainly due to the LR stage. To address this issue, the proposed implementation has been developed using a GPU since it provides a huge capability of parallel processing and rapid prototyping.

A comparison between the improved GPU implementations of the algorithms under study and their CPU counterparts is carried out, showing that the GPU considerably speeds up the execution of the algorithms. GPUs also allow for reconfigurability and this advantage has been exploited to propose the reconfigurable THP scheme. Moreover, since the GPU is more seldom used than the CPU in 


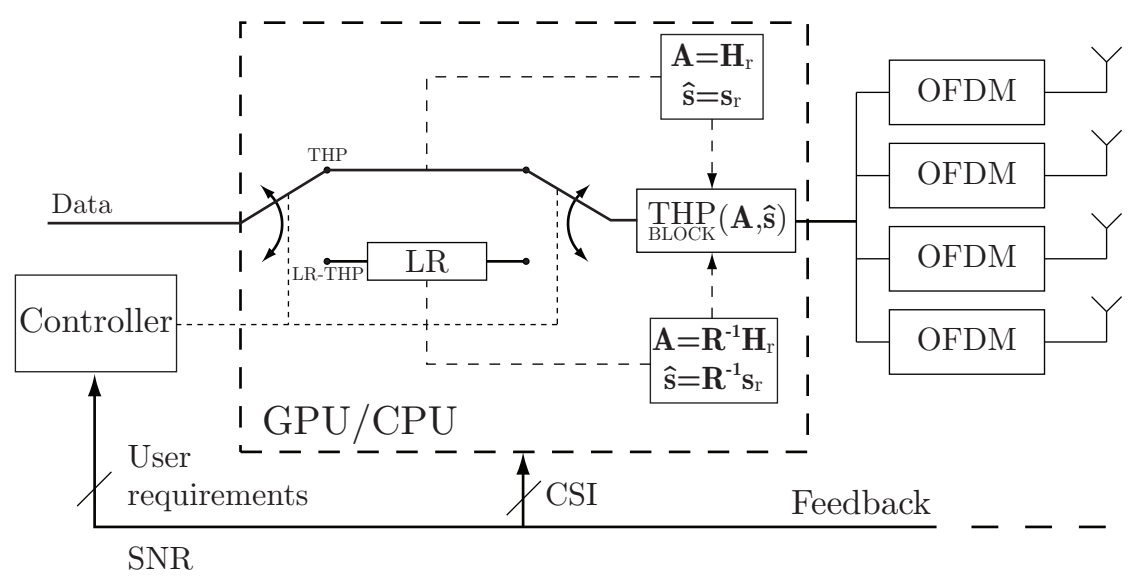

Figure 5.6: Reconfigurable THP scheme.

conventional applications, its use as a co-processor in signal processing systems is very promising.

\subsubsection{Reconfigurable THP scheme}

Figs. 3.8-3.11 show that the THP algorithm can be substantially improved by first performing an LR over the channel matrix. However, depending on the user requirements and channel conditions, the performance of THP can be sufficient with a much lower computational cost than LR-THP. Thus, a reconfigurable scheme that can switch between the conventional THP algorithm and LR-THP is proposed and depicted in Fig. 5.6.

As reported in [241], reconfigurable approaches are meaningful since they allow a flexible trade-off between the energy efficiency and QoS. Note that, through a switch, the original data $\mathbf{s}_{\mathrm{r}}$ is replaced by the modified data $\mathbf{R}^{-1} \mathbf{s}_{\mathrm{r}}$, allowing the use of a reduced channel matrix $\widetilde{\mathbf{H}}_{\mathrm{r}}=\mathbf{R}^{-1} \mathbf{H}_{\mathrm{r}}$ in the THP block.

\subsubsection{Implementation details}

The Nvidia Tesla C2070 GPU has been used for the reconfigurable scheme. Table 5.4 presents the specifications of the device. The architecture of this GPU is Fermi and hence, it supports a high parallelism level with several kernel execution overlapping, data copy and kernel execution overlapping, simultaneous host to device and device to host data copy, etc. The installed CUDA toolkit and SDK version is 4.0 [237]. 
Table 5.4: Nvidia Tesla C2070 features.

\begin{tabular}{|l|l|}
\hline Compute capability & 2.0 \\
\hline Number of SM & 14 \\
\hline Number of CUDA cores & 448 \\
\hline Clock rate & $1.15 \mathrm{GHz}$ \\
\hline Global memory & $4 \mathrm{~GB}$ \\
\hline Constant memory & $64 \mathrm{kB}$ \\
\hline Shared memory per block & $48 \mathrm{kB}$ \\
\hline
\end{tabular}

In this case, a single kernel is employed for the implementation of either the THP or the LR-THP algorithm, reducing the overhead related to kernel executions. The initial input data will differ depending on the selected algorithm. A twodimensional grid with two-dimensional blocks is considered. The blocks have 16 threads per dimension, such that $N_{\mathrm{T}}=256$. Since the channel is considered to remain constant during $L_{\mathrm{ch}}=20$ time intervals, each thread block will be in charge of the processing of a subgroup of subcarriers associated with these number of intervals. Thus, the number of subcarriers to be processed by each block can be software-defined at the beginning as $N_{\text {sub }}=\left\lfloor N_{\mathrm{T}} / L_{\mathrm{ch}}\right\rfloor$. For the case considered in this section $N_{\text {sub }}=12$ subcarriers/block. Then, for a certain number of subcarriers the number of blocks per dimension is obtained as $N_{\mathrm{B}}=\left\lceil\sqrt{K / N_{\text {sub }}}\right\rceil$.

Before starting the process, the channel matrices and signals to be precoded $(\mathbf{H}[k], \mathbf{s}[k])$ associated with $L_{c h}$ time intervals and $K$ subcarriers are stored in the GPU global memory. The preprocessing stage of the algorithm is executed by one thread per subcarrier and its output data (matrices $\mathbf{L}, \mathbf{Q}^{\dagger}$ and, only for the LR-THP case, $\mathbf{R}^{-1}$ ) are stored in the GPU shared memory, which allows a faster access. After this, all threads are synchronized to fetch the data from shared memory and start the per-symbol-vector processing stage using one thread per symbol-vector. Vector $\mathbf{x}$ is obtained and stored temporally in the registers and, at the end, copied to global memory for its output.

\subsubsection{Results}

In this section, the parallel implementation of THP and LR-THP algorithms on a GPU is compared to their implementations in a high-performance CPU. The selected CPU is an Intel Xeon X5680 at $3.33 \mathrm{GHz}$ with $96 \mathrm{~GB}$ of DDR3 main memory and $12 \mathrm{MB}$ of cache memory running Linux. The compiler used is Intel C compiler with the global performance optimization -o3.

Figure 5.7 shows the speed-up for $N_{t}=M=4$ using double precision and different number of subcarriers, $K$. It can be observed that, while the GPU-based 


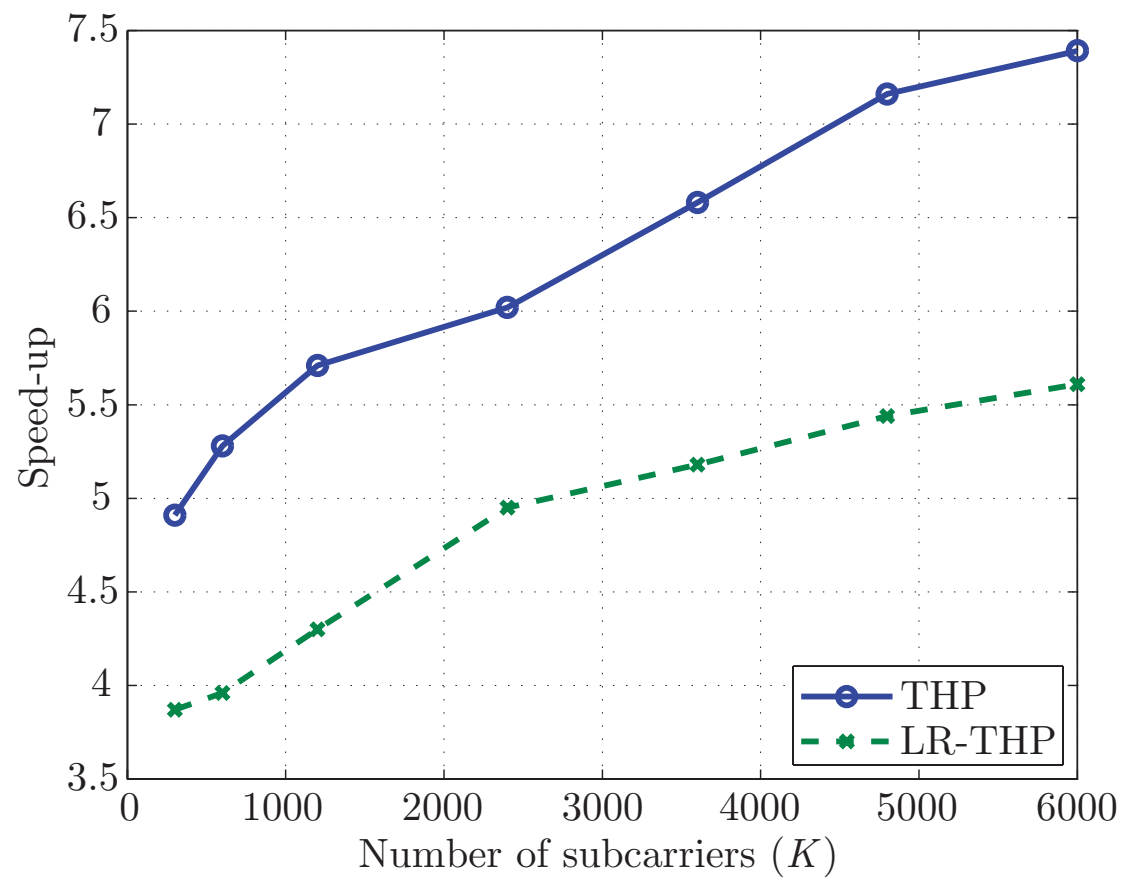

Figure 5.7: Speed-up of THP and LR-THP schemes on GPU compared to CPU for $L_{\mathrm{ch}}=20$ and $N_{t}=M=4$.

THP implementation performs up to 7 times faster than its CPU counterpart, the LR-THP implementation reaches a speed-up up to 5.5. Both speed-ups are quite promising, but it would be interesting to investigate the speed-up reduction effect experienced by the LR-THP with respect to the THP. For this purpose, the speed-up achieved by the implementation of only the LR stage was evaluated independently for the same system configurations previously discussed. Results showed that this partial speed-up ranges between 2 and 3, meaning that this stage acts as a bottleneck for the implementation of the LR-THP.

As described in section 5.1, any flow control instruction can significantly impact the effective instruction throughput achieved by the GPU due to warp divergence. Indeed this is what happens when the LR is applied over different channel matrices $\mathbf{H}[k]$, since the LLL method contains two if statements dependent on the processed channel matrix (see Algorithm 1 in section 2.7). This situation causes the serial execution of the different paths, thus increasing the total number of instructions executed for this warp and consequently the computational time. This problem could be partially alleviated when correlation between subcarriers exists, since the execution path can be similar for highly correlated matrices. However, in this case, 


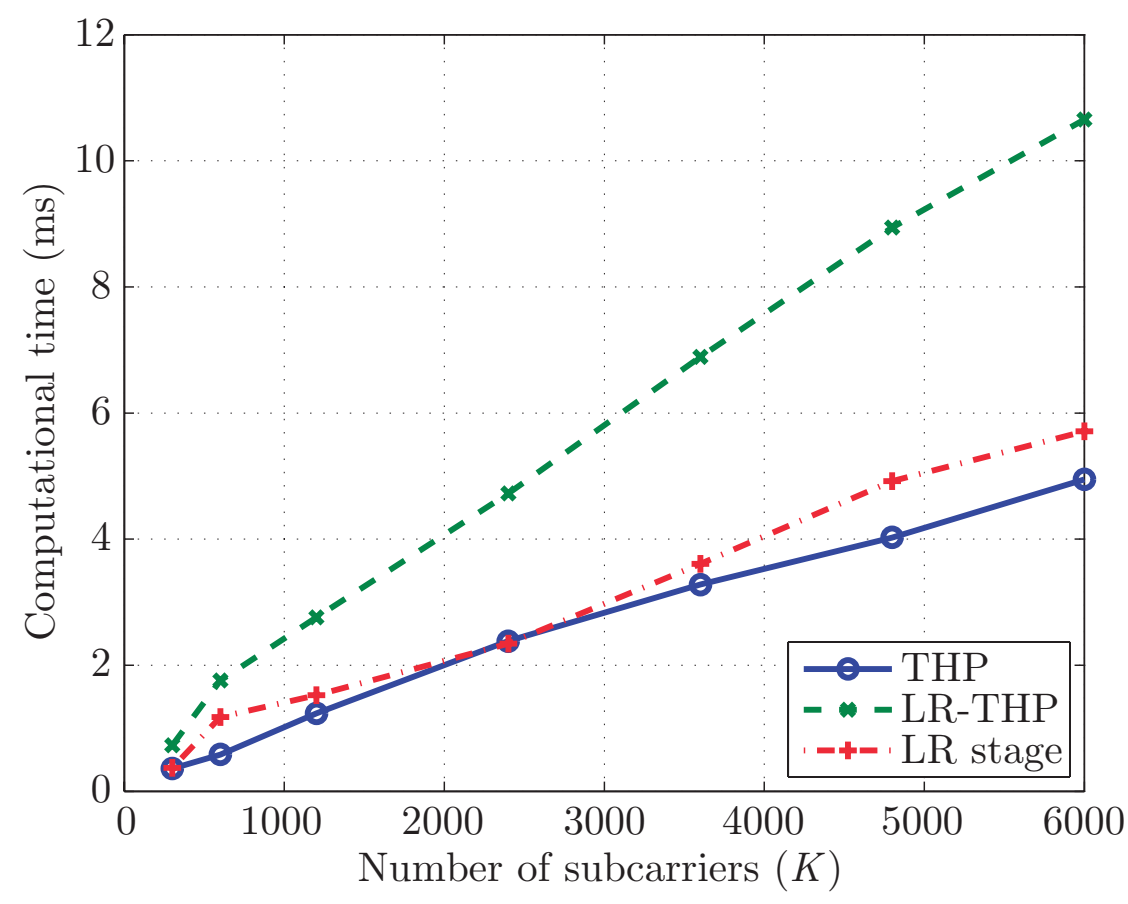

Figure 5.8: Computational time of THP, LR-THP and the LR-stage on GPU compared to CPU for $L_{\mathrm{ch}}=20$ and $N_{t}=M=4$.

uncorrelated channel matrices are considered, and therefore, the implementation evaluation is performed considering the worst case.

In addition, the computational time of the THP and LR-THP schemes on a GPU is depicted in Fig. 5.8. It can be seen that LR-THP has a considerably higher execution time than THP, increasing the difference between them as the number of subcarriers increases. Observing the execution time of the LR-stage used in LRTHP scheme, it can be observed that this stage takes even longer than $L_{\text {ch }}=20$ runs of the THP. This result again shows that the LLL algorithm is not as suitable as the THP scheme for a GPU implementation. 


\subsection{Parallelization of the LLL algorithm on GPU}

The previous sections showed that LR is the main bottleneck in the implementation of LR-THP in a multi-carrier system. Until now, only the multi-carrier parallelism had been considered. In this section, the parallelization of the LR problem itself is proposed. This result can be used to leverage different levels of parallelism in multi-carrier systems.

Several hardware implementations of the LLL algorithm can be found in the literature. Implementations that make use of LR to improve the detection performance of multiple antenna systems can be found in [242-246]. In [242] an LR-aided symbol detector for MIMO-OFDM is implemented using $65 \mathrm{~nm}$ application-specific integrated circuit (ASIC) technologies. In [243] a field-programmable gate array (FPGA) implementation of a variant of the LLL algorithm, the Clarkson's algorithm, is presented. Its main benefit is the complexity reduction without significant performance loss in MIMO detection. In [244] a hardware-efficient very-large-scale integration (VLSI) architecture of the LLL algorithm is implemented, which is used together with SIC in MIMO data detection. More recently, [245] makes use of a Xilinx XC4VLX80-12 FPGA for implementing LR-aided detectors, whereas [246] uses an efficient VLSI design with a pipelined architecture capable of sustaining a throughput of $880 \mathrm{Mb} / \mathrm{s}$ for detecting 64-QAM symbols in a 4x4 MIMO system.

This section presents a low level, fine-grained parallelism LR algorithm, the costreduced all-swap LLL (CRAS-LLL) algorithm. Its low processing time is assured by an efficient work distribution, minimizing the idle time of the launched threads [230]. Based on the parallel block-reduction concept presented in [247], a higher level, coarse-grained parallelism is presented as an extra level of parallelism. The idea is to subdivide the original lattice basis matrix in several smaller submatrices and perform an independent LR on them followed by a boundary check between adjacent submatrices [231]. The independent LR over the submatrices is performed using the CRAS-LLL algorithm. The implementation of the algorithms is carried out on several architectures including multi-core CPU, GPU and a heterogeneous platform consisting of CPU+GPU. The nomenclature used in this section can be found in section 2.7.

\subsubsection{Introduction to parallel lattice reduction strategies}

Since the LLL algorithm shows a highly sequential behavior, multiple levels of parallelism have to be identified and explored in order to parallelize this algorithm. Previous parallel LR implementations, such as the ones presented in [248-250], have only focused on multi-core architectures. Their main drawback is the low number of threads and the limited parallelization possibilities offered by these architectures compared to the GPU architectures. If the problem could be divided into several sub-problems and these sub-problems could benefit from a multi- 
threaded environment, the low number of threads offered by the multi-cores would represent a significant limiting factor. In the case of GPUs, the high number of CUDA cores makes the parallel execution of a high number of threads possible, thus making the usage of a multi-level parallelism feasible.

\subsubsection{Cost-reduced all-swap LLL algorithm}

The order in which the SwAP procedure is applied in the LLL algorithm (Algorithm 1) is very limiting when implemented within a parallel framework. The concept of any swap reduction, which was introduced in [251], enables simultaneous basis swaps and serves as a basis for future parallel implementations. Further computational cost can be saved by rearranging and delaying the frequently used SizeREDUCTION procedure [248].

The procedures SimpleSizeReduction, SimpleSwap and Swap are defined in order to give an accurate description of the CRAS-LLL and the following algorithms.

Procedure 1 SimpleSizeReduction $(\mathbf{B}, k, l)$ Given a lattice generator matrix $\mathbf{B}$ and the associated GSCs matrix $\mathbf{U}$, if the condition (2.32) is not satisfied, i.e. $\left|\mu_{k, l}\right|>\frac{1}{2}$, the following updates have to be applied:

- $\mu=\left\lceil\mu_{k, l}\right\rfloor, \mu_{k, l}=\mu_{k, l}-\mu, \mathbf{b}_{k}=\mathbf{b}_{k}-\mu \mathbf{b}_{l}$.

Procedure 2 SimpleSwap $(\mathbf{B}, k)$ Given a lattice generator matrix $\mathbf{B}$, the associated orthogonal basis $\mathbf{B}^{*}$ and GSCs matrix $\mathbf{U}$, if the condition (2.33) is not satisfied, or equivalently $\left\|\mathbf{b}_{k}^{*}\right\|^{2}<\left(\delta-\mu_{k, k-1}^{2}\right)\left\|\mathbf{b}_{k-1}^{*}\right\|^{2}$, the following updates have to be applied:

- $\operatorname{swap} \mathbf{b}_{k}$ with $\mathbf{b}_{k-1}$

- $\mathbf{b}_{k-1}^{* \text { । }}=\mathbf{b}_{k}^{*}+\mu_{k, k-1} \mathbf{b}_{k-1}^{*}, \mu_{k, k-1}^{\prime}=\left(\mathbf{b}_{k-1}^{*}, \mathbf{b}_{k-1}^{* 1}\right) /\left\|\mathbf{b}_{k-1}^{* 1}\right\|^{2}$,

$$
\mathbf{b}_{k}^{* 1}=\mathbf{b}_{k-1}^{*}-\mu_{k, k-1}^{\prime} \mathbf{b}_{k-1}^{* 1}
$$

- $\mathbf{b}_{k-1}^{*}=\mathbf{b}_{k-1}^{* 1}, \mathbf{b}_{k}^{*}=\mathbf{b}_{k}^{* !}, \mu_{k, k-1}=\mu_{k, k-1}^{1}$

Procedure $3 \operatorname{SwAP}(\mathbf{B}, k)$ Given a lattice generator matrix $\mathbf{B}$, the associated orthogonal basis $\mathbf{B}^{*}$ and GSCs matrix $\mathbf{U}$, if the condition (2.33) is not satisfied, or equivalently $\left\|\mathbf{b}_{k}^{*}\right\|^{2}<\left(\delta-\mu_{k, k-1}^{2}\right)\left\|\mathbf{b}_{k-1}^{*}\right\|^{2}$, the following updates have to be applied: 
- $\operatorname{swap} \mu_{k, j}$ with $\mu_{k-1, j}$, for $1 \leq j<k-1$,

- $\left(\begin{array}{c}\mu_{i, k-1} \\ \mu_{i, k}\end{array}\right)=\left(\begin{array}{c}\mu_{i, k-1} \mu_{k, k-1}^{\prime}+\mu_{i, k}\left\|\mathbf{b}_{k}^{*}\right\|^{2} /\left\|\mathbf{b}_{k-1}^{* !}\right\|^{2} \\ \mu_{i, k-1}-\mu_{i, k} \mu_{k, k-1}\end{array}\right)$ for $k+1 \leq i<n$.

In Algorithm 2, a detailed description of the CRAS-LLL algorithm is presented. This algorithm combines the any swap strategy with the size reductions strategy [230]. The performance of the CRAS-LLL algorithm basically depends on the efficiency of the work distribution, inner products, size reductions and column swappings.

Figure 5.9 depicts the implementation details for the main parts of the algorithm. The work distribution is simple to solve and it is highly parallel. In order to get an efficient utilization of the threads, a 2 dimensional thread block configuration is proposed, $\operatorname{TB}\left(T_{x}, T_{y}\right)$. The $y$ dimension is defined based on the size of the original basis, namely $T_{y}=\min (m / 2,32)$, where the maximum limit is selected for optimization reasons. By enabling the usage of $T_{x}=\min (m, 32)$ threads in the $x$ dimension, the threads that belong to the same $y$ dimension will form a warp, and consequently, the global memory loads and stores issued by the threads of the warp will be coalesced. Thus, the size reductions, inner products and column swaps can be computed in a more efficient manner, exploiting the advantages of the caching system and the low latency of the shared memory [230].

Algorithm 8 in Appendix A shows the CUDA pseudo-code to facilitate the GPU implementation. In this algorithm, the $y$ dimension also defines the extent of parallelism. The iteration variable of the for loop is increased in every iteration by $2 T_{y}$. In other words, in every phase the threads with the same $y$-index have to reduce and swap at most $m /\left(2 T_{y}\right)$ vectors. Matrices $\mathbf{B}, \mathbf{B}^{*}, \mathbf{U}$ are stored in the global memory of the GPU. This memory has a high latency, but with a coalesced access pattern, optimal memory usage can be achieved. Depending on the basis dimension, the usage of the shared memory is also possible for storing matrices $\mathbf{B}, \mathbf{B}^{*}, \mathbf{U}$. The shared memory is also used to store the GSCs $\mu_{i, i-1}$ and to create two buffer arrays that will help compute the inner products and size reductions. When computing the inner product, the elements of $\mathbf{b}_{k}$ are read in a coalesced pattern and each thread will sum the corresponding elements in the shared memory buffer. After the sum, the parallel prefix sum pattern is applied to the buffer resulting in the inner product value. In the case of a size reduction, the corresponding elements are reached in a coalesced pattern and the corresponding $\mu_{k, k-1}$ is read from the shared memory. Because the threads belonging to the same $y_{t i d}$ will access the same $\mu_{k, k-1}$ in the shared memory, the result will consist of a memory broadcast instead of a bank conflict [231].

Algorithm 9 in Appendix A shows the implementation of the CRAS-LLL algorithm for multi-core GPU using OpenMP [252]. Two-level parallelism is implemented based on a nested parallelism construct. The outer level parallelism starts the 


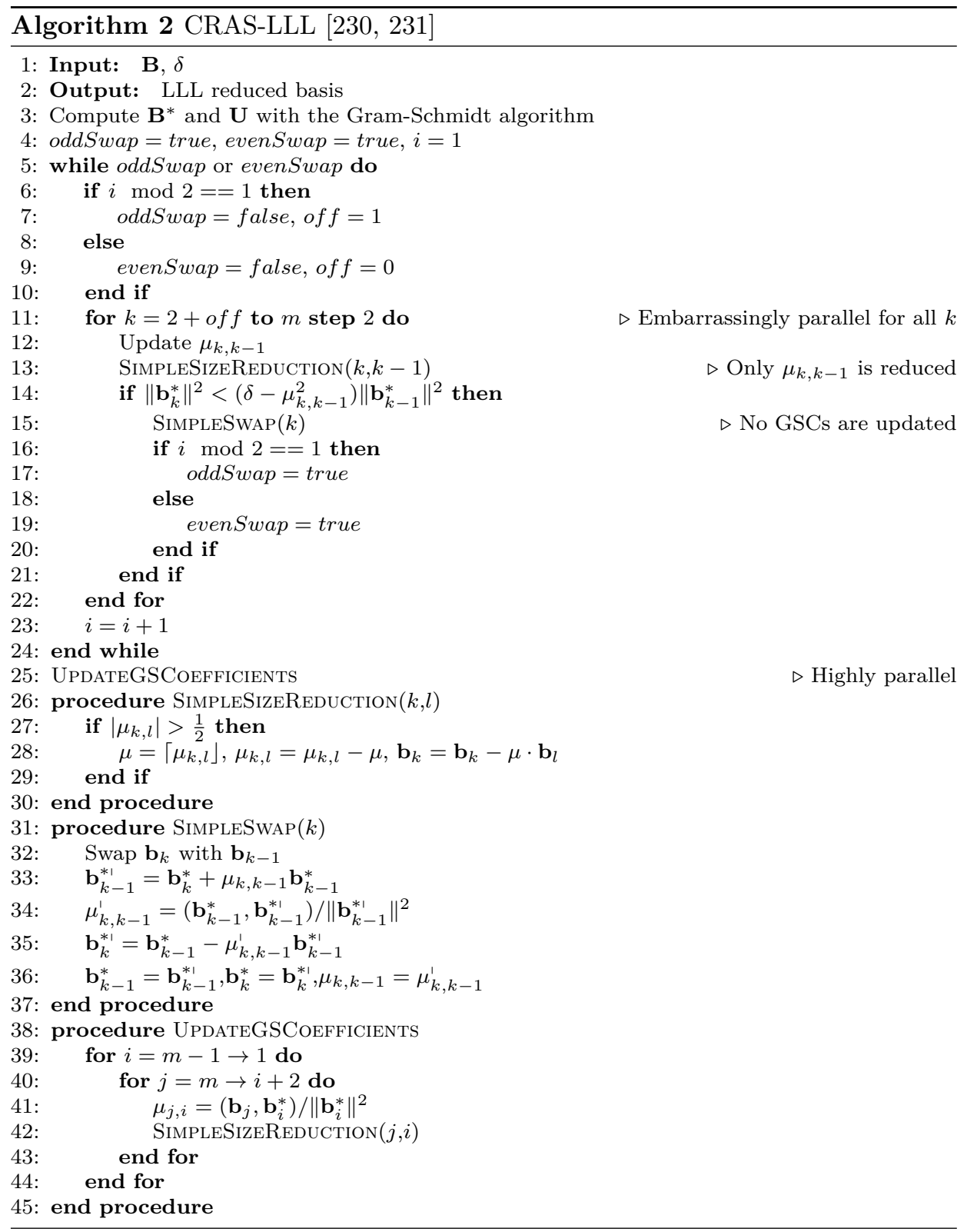



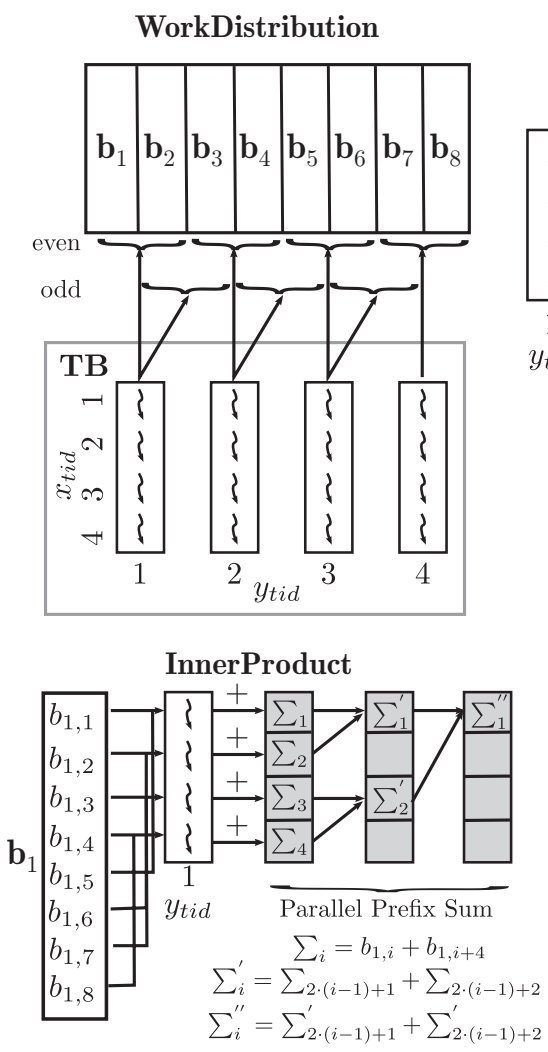

SizeReduction

$\mathbf{b}_{k}=\mathbf{b}_{k}-\mu_{k, k-1} \mathbf{b}_{k-1}$

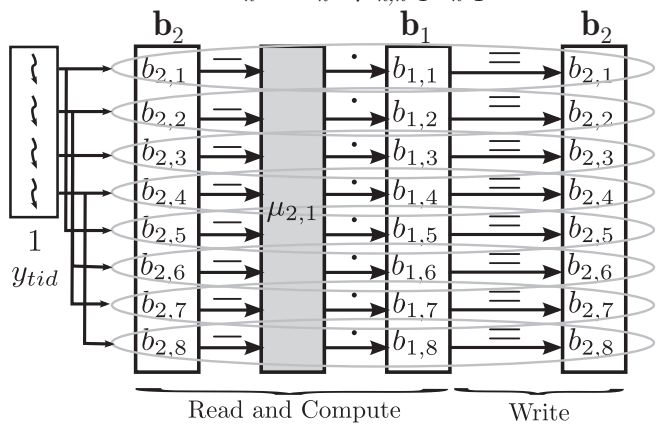

\section{Swap}

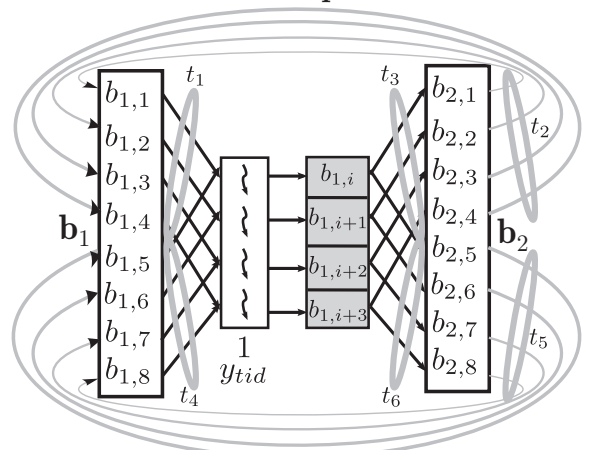

Figure 5.9: CRAS-LLL algorithm mapping to GPU architecture

concurrent processing of simMat number of lattice basis and the inner parallel construct is responsible for the parallel LR of a basis with TPM number of threads. As the outer level parallelism is expanded, simMat is increased and the threads available for the parallel LR are decreased.

\subsubsection{Modified block LLL}

This section presents the modified block LLL (MB-LLL) algorithm, which splits the original matrix basis into several submatrices of lower dimension in a block-wise manner and performs the CRAS-LLL on them. Once the LR of the submatrices is finished, the boundaries between adjacent submatrices are checked and finally the GSCs outside the initial groups are updated. The main condition is to keep every submatrix as an LLL-reduced matrix throughout the processing [231]. 


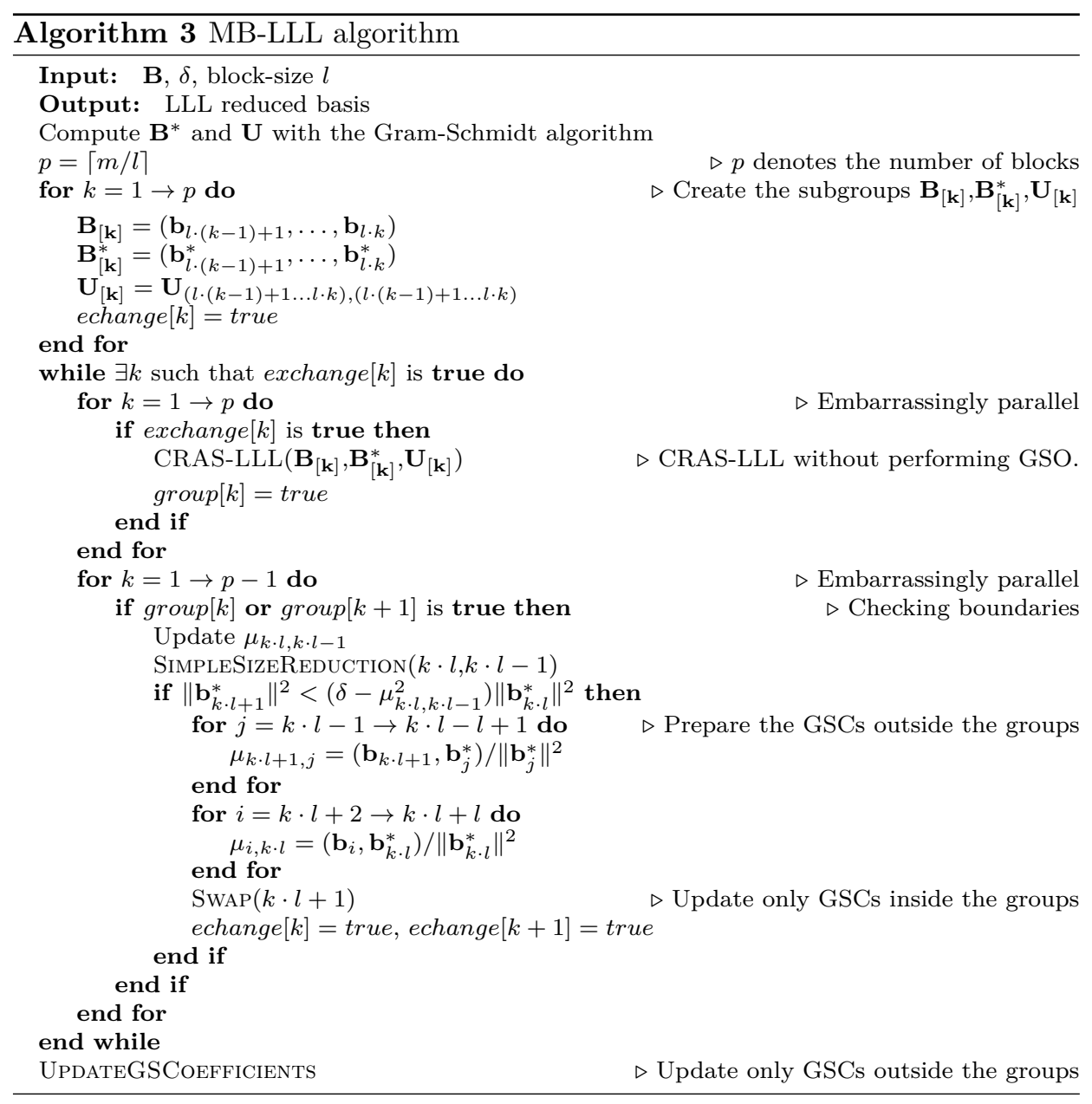

The details of MB-LLL are given in Algorithm 3. Since the LLL reduction of the subgroups and the boundaries check can be done independently, no frequent synchronization is required. Thus, coarse grained parallelism can be achieved by working on the sub-problems. 


\subsubsection{Cost-reduced modified block LLL}

This section presents the cost-reduced modified block LLL (CRMB-LLL) algorithm in order to further reduce the computational complexity of the MB-LLL algorithm. The main idea behind the CRMB-LLL algorithm is the relaxation of the first LLL condition while executing the LR for the submatrices, resulting in the delay of the GSCs update and using less costly procedures when performing the boundary checks [232].

In the MB-LLL algorithm, the submatrices affected by a boundary swap have to be LLL-reduced and the GSCs have to be updated. Moreover, in order to maintain the LLL conditions in the submatrices affected by a boundary swap, the SwAP procedure has to be performed. The computational reduction is achieved by eliminating the GSCs update in the submatrices after the execution of the CRASLLL and the usage of the SimpleSwAP procedure instead of SWAP in case of a boundary swap. Since the GSCs are updated only when the ordering condition (2.33) is met for every column vector, the processing time can be considerably reduced.

\subsubsection{Implementation of MB-LLL and CRMB-LLL}

The performance of the MB-LLL and CRMB-LLL algorithms is evaluated for different architectures: a heterogeneous platform consisting of a CPU and a GPU is proposed and it is compared with implementations running on a GPU with dynamic parallelism (DP) capability and a multi-core CPU architecture. The algorithm mappings on different parallel architectures is very challenging, since the number of processing cores, latency and size of cache and other different memories available differ significantly.

\section{DP-based GPU}

The implementation of these algorithms can make use of new features introduced in CUDA 5.0, such as DP [253]. This feature allows launching new kernels and synchronizing from the GPU without returning the control to the CPU. DP is only supported by devices of compute capability 3.5 and higher. Figure 5.10 presents the scheme of the MB-LLL kernel, but it is also valid for the CRMB-LLL algorithm. The CPU launches the $M B-L L L$ Kernel that is responsible for:

- Passing the correct data and launching the CRAS-LLL kernels in order to perform the LLL reduction of the sub-problems.

- Launching the Boundaries Check kernels for checking the LLL conditions at the boundaries of the sub-groups. 


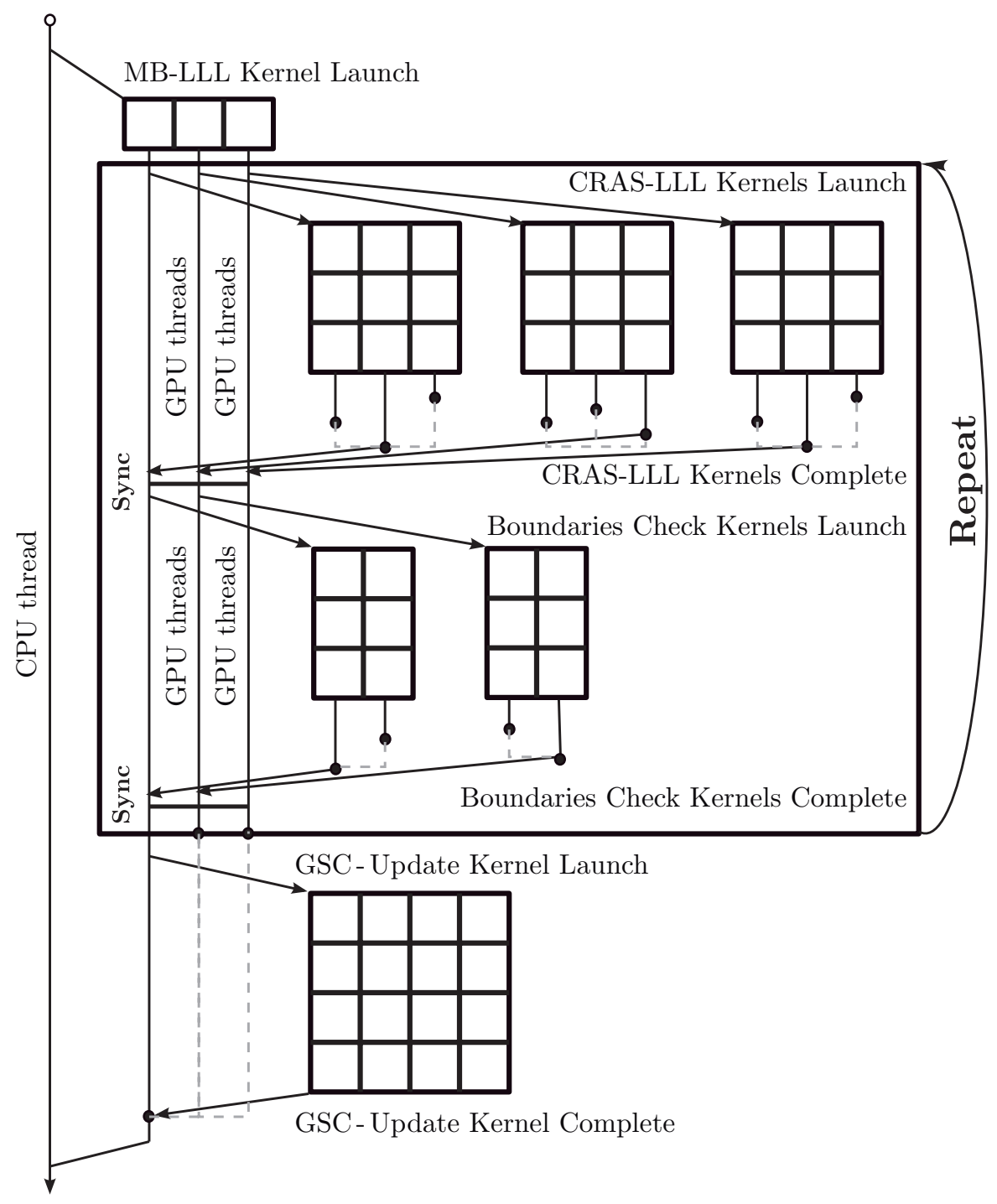

Figure 5.10: Kernels scheduling for the MB-LLL algorithm.

- Launching the GSC-Update kernel to update the GSCs coefficients and to perform the size reductions wherever it is required.

It can be seen that synchronization of the threads is necessary only after finishing the LR of the sub-problems and after the boundary check. The size of the TBs is different for each kernel because the tasks that have to be performed are different 
as well. Through the efficient work distribution and the emphasized use of the parallel design patterns, significant speed-up can be achieved compared to existing results.

The size of the grid is equal to the number of matrices that are simultaneously processed and the number of threads in one TB is equal to the number of submatrices. Every thread has to prepare the data for the corresponding submatrices and launch the CRAS-LLL kernel. The kernel has to be re-launched if the LLL conditions were broken by a boundary swap, which can be solved by tracking state variables placed in the global memory. When all the submatrices are reduced, the Boundaries Check kernel is launched. Since the operations performed in this section are dot products and column swaps, the thread configuration of the TB is the same as in case of the CRAS-LLL kernel.

The CRAS-LLL and Boundaries Check kernels are repeated until there are no swaps on the boundaries. Because one matrix is assigned to one TB in the parent $M B$ - $L L L$ kernel, the processing of the different matrices can be done simultaneously despite the variable number of iterations. Finally, the GSCs outside the blocks are updated with the $G S C$-Update kernel and the size-reduction is performed wherever is needed.

\section{Heterogeneous platform $C P U+G P U$}

The schematic of the heterogeneous platform is shown in Fig. 5.11. The multiple CPU OpenMP threads are responsible for launching CRAS-LLL, Boundaries Check and GSC Update kernels, updating the state variables and implementing the control logic of the dynamic scheduling. A comprehensive overview of OpenMP can be found in [252]. A different CUDA stream is assigned for every CPU thread, making possible the concurrent kernel execution and reducing the idle time of the CUDA cores [237]. The proposed architecture allows a dynamic scheduling of kernels where the overhead introduced by host-device communication is hidden by the use of CUDA streams.

The mapping details of the CRMB-LLL algorithm on the heterogeneous platform is presented in Algorithm 4. Before launching the CRAS-LLL and Boundaries Check kernels, the CPU thread updates the matIndD array placed in the GPU's global memory to specify which matrices need further processing. The size of the grid is dynamically adjusted according to the number of non-processed matrices in every iteration. After the Boundaries Check kernel is executed and the boundaryExchH is updated on the host, the CPU thread checks if the LR of any matrix has finished. If LLL reduced matrices are found, the matIndH is updated and consequently the size of the grids assigned to the CRAS-LLL and Boundaries Check kernels is decreased. The GSC update kernel starts after all the matrices assigned to one CPU thread have been completely processed. 


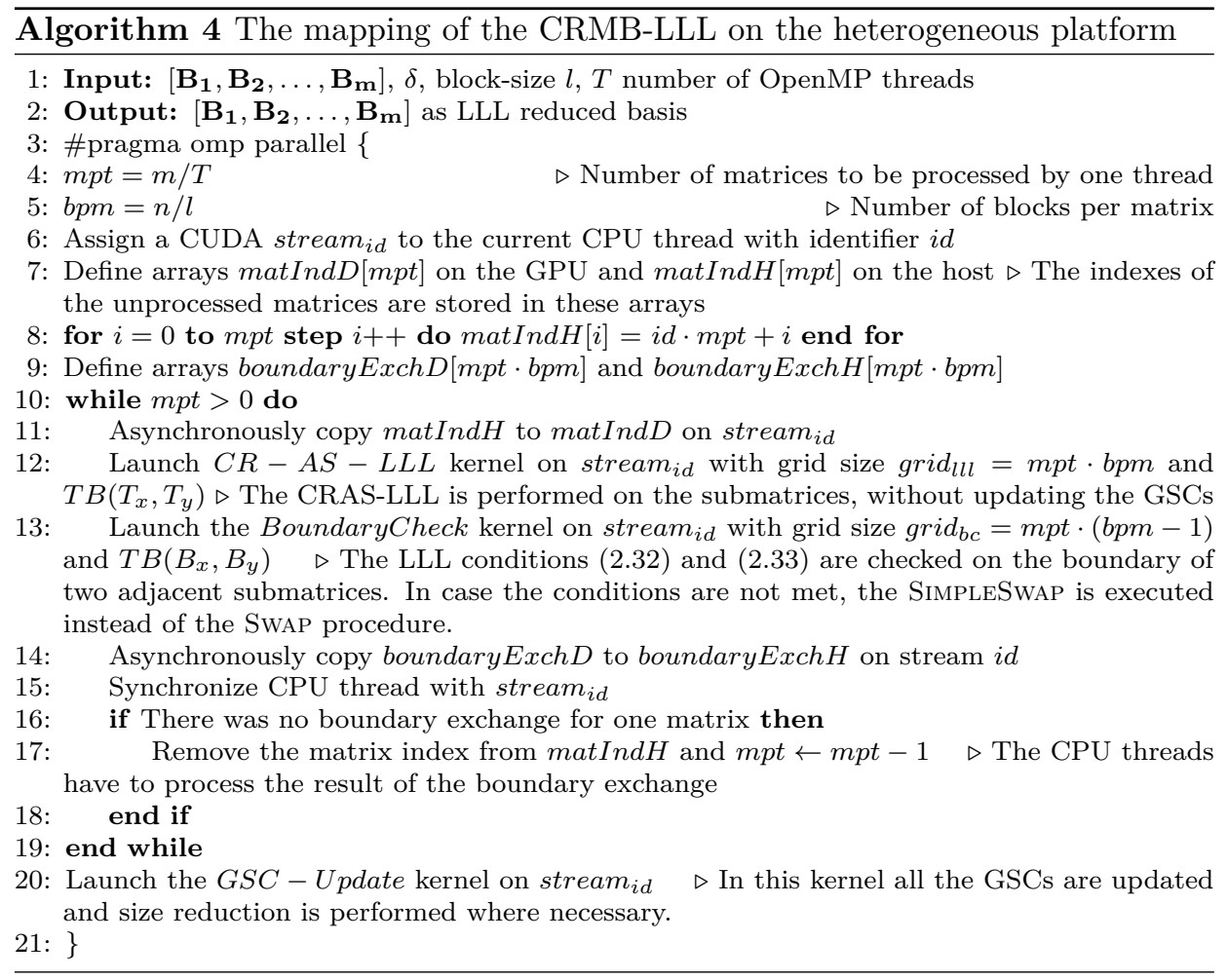




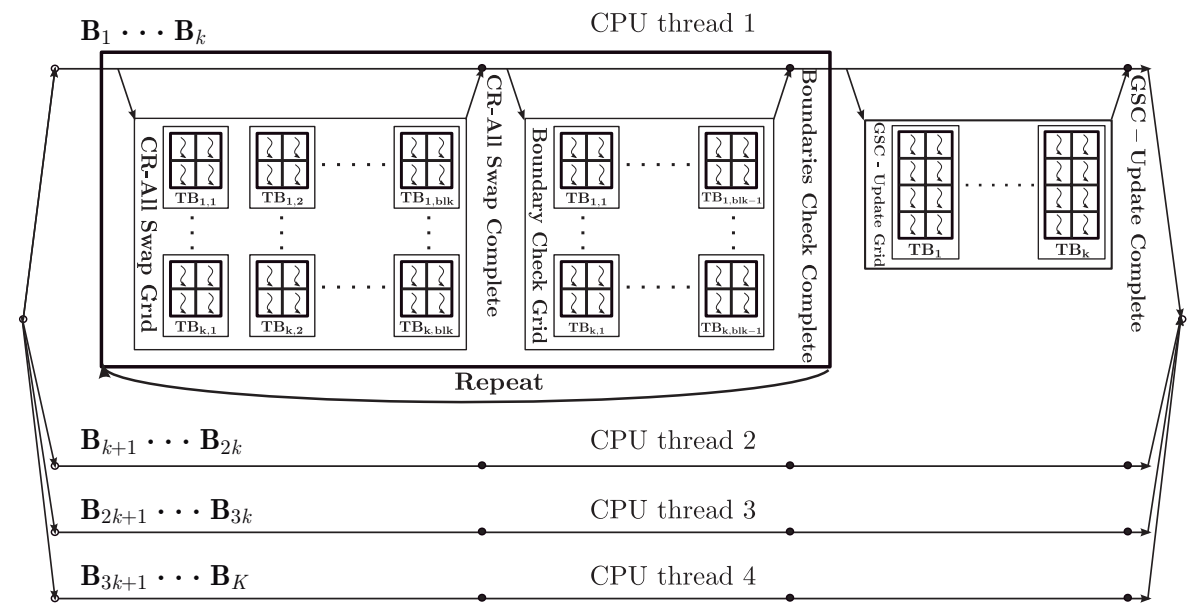

Figure 5.11: Kernels scheduling on the heterogeneous platform for the MB-LLL algorithm.

\section{Multi-core CPU}

The control structure required by the multi-core architecture is similar to the one presented in the heterogeneous platform. The difference is that instead of launching GPU kernels, the master threads fork a specified number of slave threads that are processing the submatrices in parallel. The parallel LR of the submatrices is performed according to Algorithm 9 in Appendix A, which gives the details of the OpenMP implementation of the CRAS-LLL algorithm.

Two-level parallelism is implemented based on nested parallelism. The outer level parallelism starts the concurrent processing of simMat number of lattice basis and the inner level is responsible for the parallel LR of a basis with TPM number of threads. As the outer level parallelism is expanded, simMat is increased and the threads available for the parallel LR are decreased. In this case, the very limited number of CPU threads restrict the exploitation of several levels of parallelism.

\subsubsection{Results}

This section presents results regarding the implementation of the CRAS-LLL, MBLLL, CRMB-LLL algorithms on the different architectures.

Firstly, a comparison of the CRAS-LLL implementation on different Nvidia GPUs is presented. The GPU configurations used for the comparison are: $1 \times \mathrm{K} 20,2 \times$ K20, $2 \times$ C2075 and $1 \times$ GTX690. A comparison of the hardware components of these GPUs can be found in Table 5.5. The GTX690 has the highest number of 
Table 5.5: Nvidia GTX690, K20 and C2075 GPU features.

\begin{tabular}{|c|c|c|c|c|c|}
\hline Model & Architecture & Die Count & Memory (MB) & SMs & CUDA cores \\
\hline GeForce GTX 690 & 2xGK104 & 2 & $2 \times 2048$ & $2 \times 8$ & $2 \times 1536$ \\
\hline Tesla K20 & GK110 & 1 & 5120 & 13 & 2496 \\
\hline Tesla C2075 & GF100 & 1 & 6144 & 14 & 448 \\
\hline
\end{tabular}

\begin{tabular}{|c|c|c|c|c|}
\hline Model & Clock rate $(\mathrm{MHz})$ & GFLOPS $($ FMA) & TDP $(\mathrm{W})$ & Price \\
\hline GeForce GTX 690 & 915 & $2 \times 2810$ & 300 & $\sim \$ 1100$ \\
\hline Tesla K20 & 705 & 3519 & 225 & $\sim \$ 3000$ \\
\hline Tesla C2075 & 1150 & 1030 & 238 & $\sim \$ 2100$ \\
\hline
\end{tabular}

CUDA cores and achievable FLOPS. In the case of the C2075, the clock rate of the cores is slightly higher. However, the number of CUDA cores is significantly lower. The multi-GPU configurations are used in order to balance the die number of the K20 and C2075 GPUs with the GTX690 GPU.

Figure 5.12 compares the average computational time of one lattice basis on the above mentioned GPU configurations. Results consider square real matrices of the form of $(2.27)$ and $\delta=3 / 4$. The average computational time was computed by averaging the processing time of 8000 lattice basis, thus the same number of thread blocks were defined for the grid. The computations were done in singleprecision floating point arithmetic. The best results are achieved by the $2 \times \mathrm{K} 20$ GPU configuration, however the GTX690 performs better than $1 \times$ K20. The result achieved with the $2 \times \mathrm{C} 2075$ also outperforms the $1 \times \mathrm{K} 20$. This is a surprising result since the FLOPS achieved by the $2 \times$ C2075 GPUs are significantly lower compared to the K20 GPU. Presumably, this is mainly motivated by the different use of the L1 cache in the Kepler architecture. In addition, comparing the computational times with other implementations in the literature, it can be stated that this implementation reduces the computational time even for small matrices [243, 244].

Figure 5.13 shows the computational time of the CRAS-LLL and the MB-LLL algorithms, evaluated on the Nvidia Tesla K20 GPU. In the case of MB-LLL, different configurations regarding the block size are shown. It can be observed that the block concept used in the MB-LLL algorithm allows the reduction of the computational time for matrix sizes larger than 256. Taking into account that the processed matrices are real, this case can correspond to a system with 128 transmit or receive antennas, known as massive MIMO systems as introduced in section 2.3. Thus, GPUs have shown to possess a powerful architecture for signal processing in massive MIMO systems. 


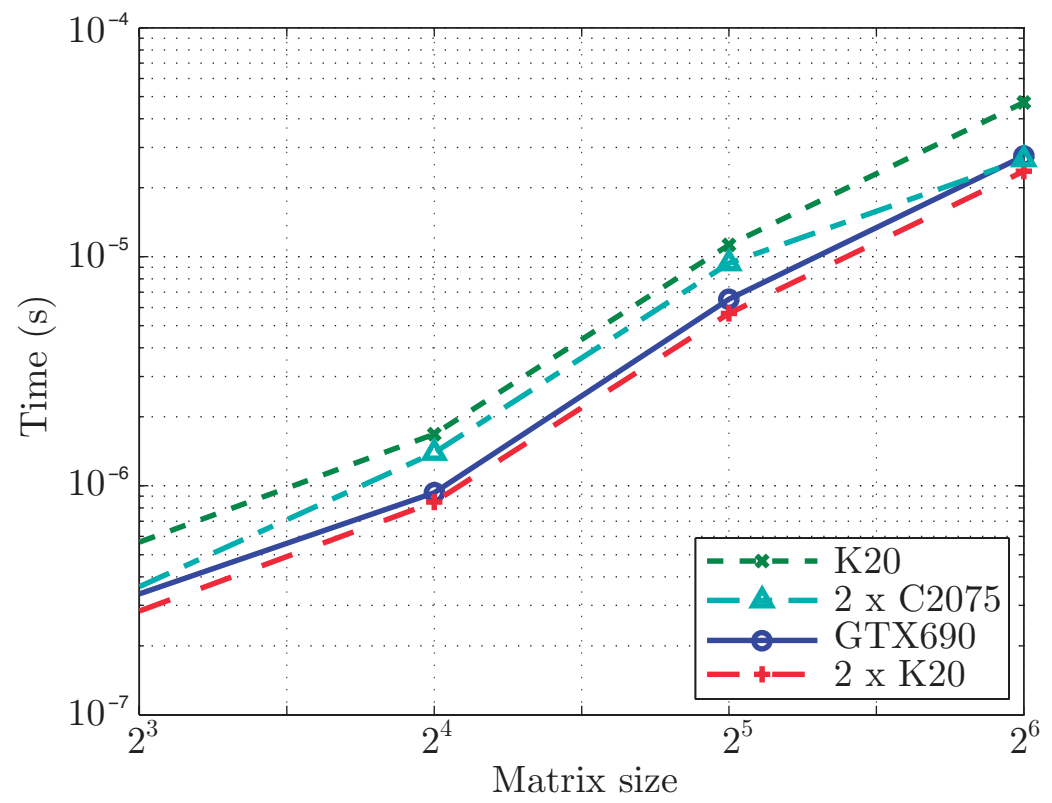

(a) Matrix size: $2^{3}-2^{6}$

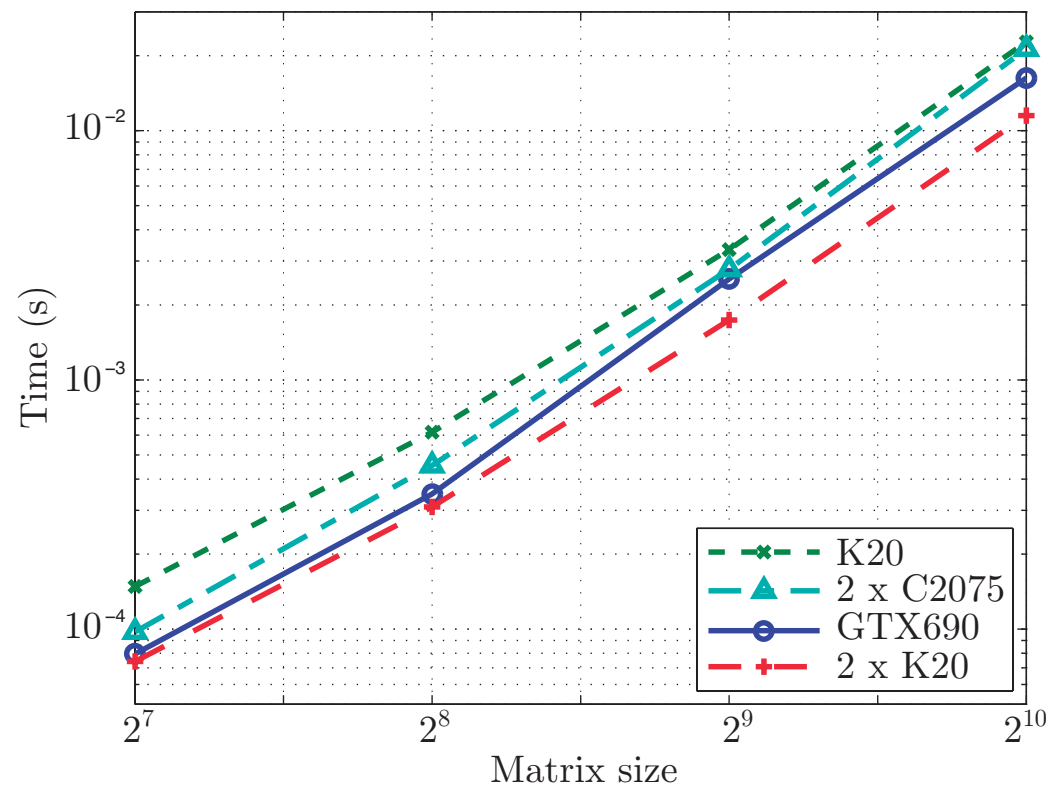

(b) Matrix size: $2^{7}-2^{10}$

Figure 5.12: Computational time of CRAS-LLL on $1 \times$ and $2 \times$ Tesla K20, $1 \times$ GeForce GTX690 and $2 \times$ Tesla C2075 GPUs for different matrix sizes. 


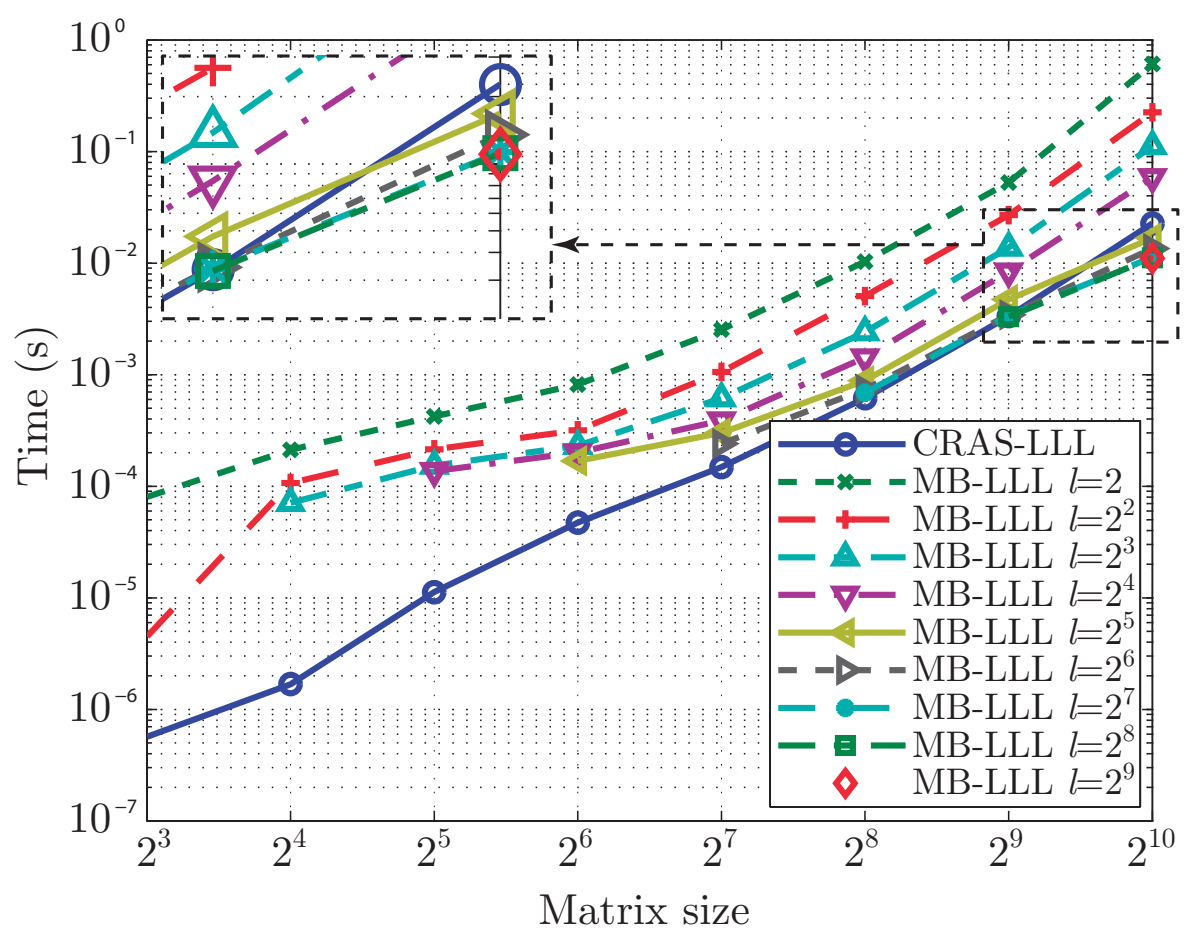

Figure 5.13: Computational time of the CRAS-LLL and MB-LLL algorithms with different block sizes, $l$, for different matrix sizes.

Figure 5.14 shows the computational times of the MB-LLL algorithm implemented on the architectures discussed in section 5.4.5 for different matrix sizes, where $l$ denotes the size of the processed blocks. The performance measurements were evaluated with all the possible block sizes and the best configuration is shown. The architectures used for the computational time measurements are the Nvidia Tesla K20 (with DP capability) and an Intel Core i7-3820 processor. The processing times show similar performance for large matrices when the GPU is involved. However, the heterogeneous platform clearly outperforms the solution based on DP in the case of small matrices. This gap is caused by the overhead required when launching CUDA kernels from CUDA kernels, without CPU involvement due to the DP feature, and the limited overlapping execution of kernels on different streams. The conclusion is that the data transfer between CPU and GPU required by the heterogeneous system is less time consuming than the overhead of the kernel launched with DP and the limitation of the concurrent execution of kernels on different streams. 


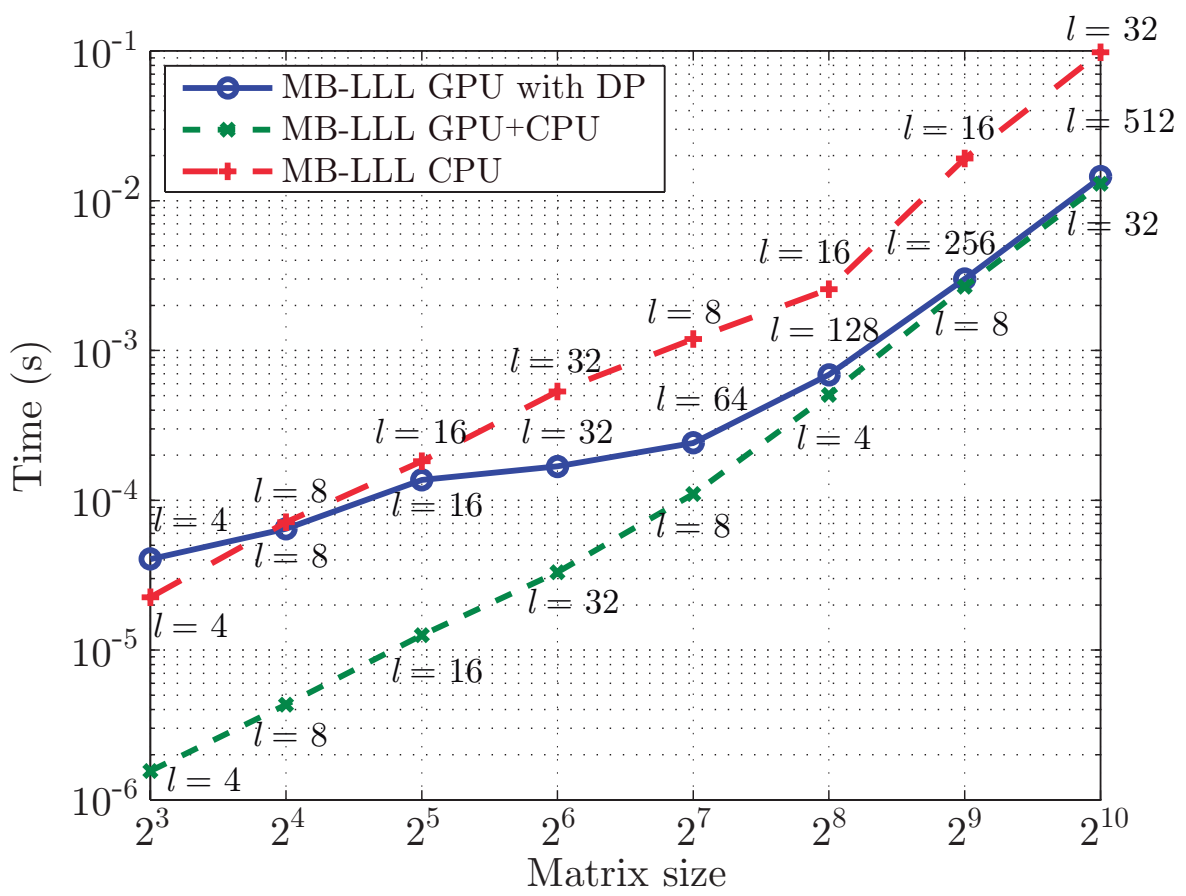

Figure 5.14: Computational time of the MB-LLL algorithm with different block sizes, $l$, on different architectures and for different matrix sizes.

Figure 5.15 compares the average computational time of the CRAS-LLL, MB-LLL and CRMB-LLL algorithms for different matrix sizes. The algorithms have also been evaluated on the Intel Core i7-3820 CPU, the Nvidia GeForce GTX 690 GPU and the heterogeneous system containing the previously mentioned CPU and GPU. Since DP does not mean any benefit as shown in Fig. 5.14, the Nvidia GeForce GTX 690 GPU has been selected instead of the K20. Regarding the GPU and combined CPU+GPU implementations, the following conclusions can be drawn:

- The computational time of the CRMB-LLL algorithm is $25-40 \%$ lower in cases of small and medium-sized matrices compared to the MB-LLL algorithm and the performance is similar with larger matrices.

- The CRAS-LLL algorithm performs better than the CRMB-LLL algorithm in case of small matrices. However, for large matrices the block concept implemented in the CRMB-LLL algorithm achieves 30\% speed-up compared to the CRAS-LLL algorithm. 


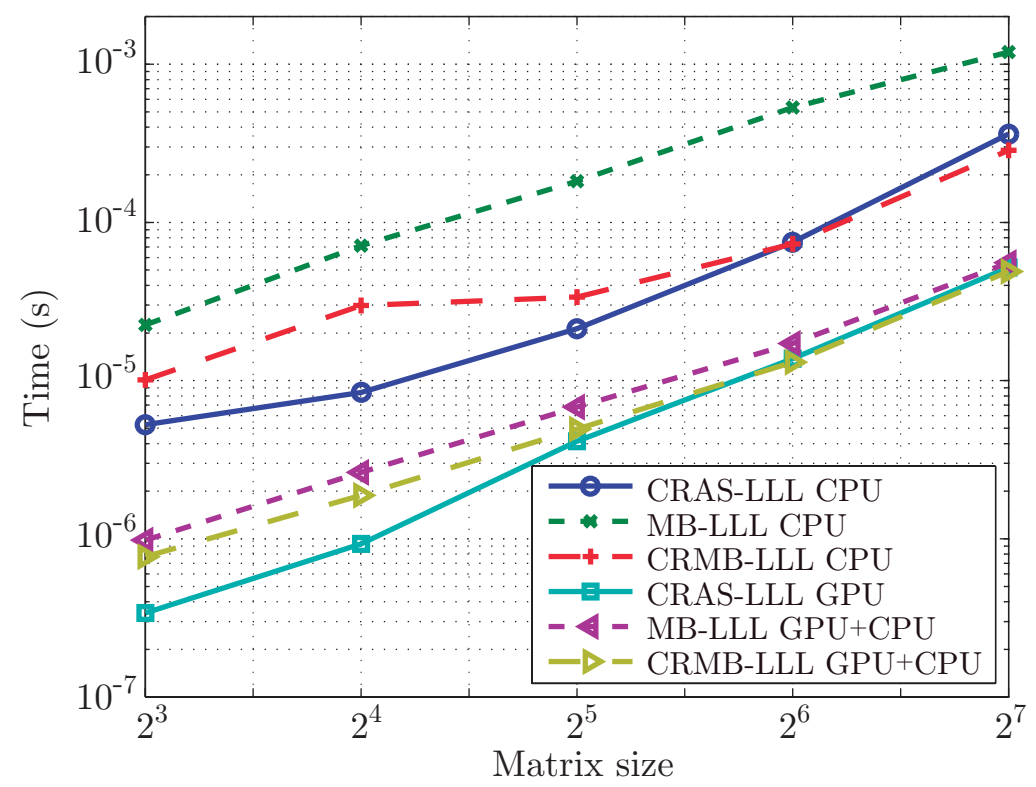

(a) Matrix size: $2^{3}-2^{7}$

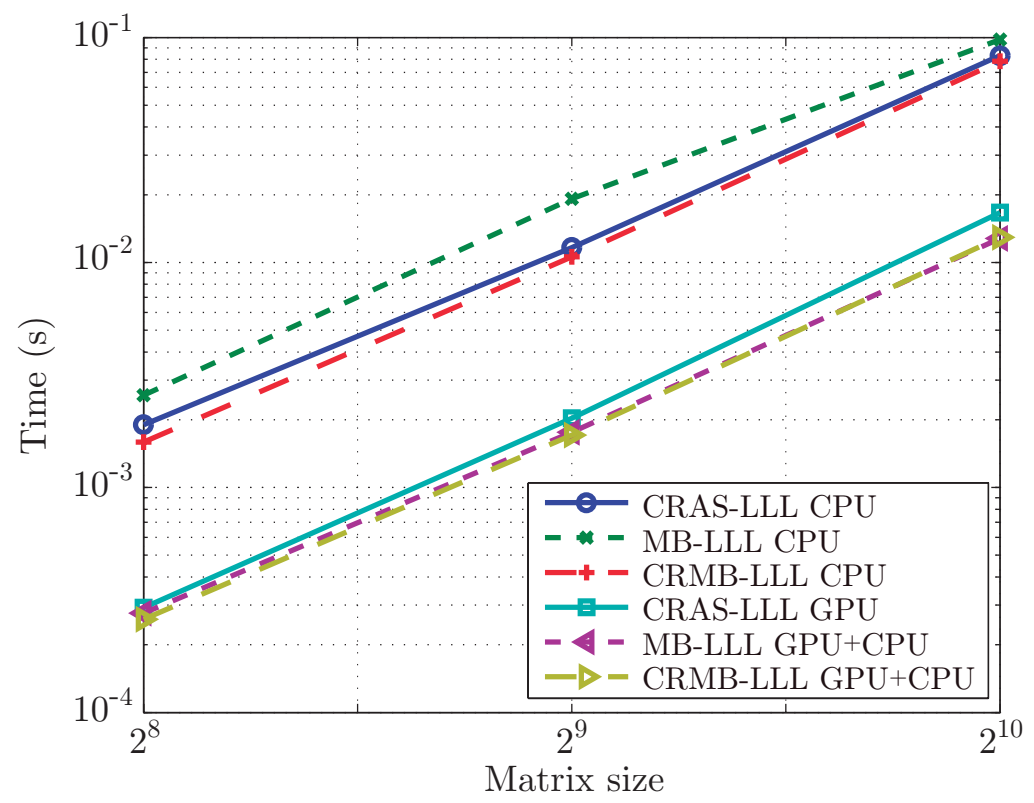

(b) Matrix size: $2^{8}-2^{10}$

Figure 5.15: Computational times of CRAS-LLL, MB-LLL and CRMB-LLL algorithms on different architectures for different matrix sizes. 
- The systems using the GPU outperforms the CPU for every matrix size with speed-ups ranging from 6 to 15 .

Regarding the CPU implementations the following conclusions can be drawn:

- The CRMB-LLL always outperforms the MB-LLL algorithm with speed-ups ranging from 2 to 7 ,

- The CRAS-LLL algorithm performs better than the MB-LLL and CRMBLLL for small matrices $\left(2^{3}-2^{6}\right)$,

- The computational time of the CRMB-LLL is 10-20\% lower in case of larger matrices compared to the CRAS-LLL.

A surprising result is that, while the CRMB-LLL achieves a significant speed-up compared to the MB-LLL for the CPU architecture, the same does not occur for the GPU architecture. This fact is due to several reasons. The computational complexity reductions for the CRMB-LLL affect only the CRAS-LLL and Boundaries Check kernels. However, in case of large matrices, the GSC-Update kernel is taking the major part of the processing time. This kernel has to access the global memory frequently and these accesses have a high latency. In case of the CPU, this problem is alleviated by the high speed memory access and the large amount of available cache.

The performance of LR mostly depends on the precision of the computation, the size and type of the basis matrix and the architecture used. In Table 5.6, the performance of existing implementations is presented. Previous research mostly focused on small matrices. In [249], performance measures for higher dimension matrices are presented as well. However, the total runtime of the algorithm is not specified.

\subsection{Conclusion}

This chapter has provided details about the GPU implementation of the precoding algorithms presented in Chapter 3 for a MU-MISO-OFDM system. The precoding stage is highly accelerated by simultaneously processing the calculations associated with all the subcarriers by forwarding their data to different threads. The GPU implementation has been compared with its CPU counterpart. The different speedup achieved by the different precoding algorithms reveals that ZF, THP and LRTHP algorithms are more suitable for a GPU implementation than the rest.

Due to the good performance of the THP implementation, a reconfigurable GPUbased implementation of the THP scheme combined with a LR stage has been 
Table 5.6: Performance comparison of different LR implementations.

\begin{tabular}{|c|c|c|c|c|c|c|}
\hline Ref & Algorithm & Architecture & $4 \times 4$ & $8 \times 8$ & $64 \times 64$ & $1024 \times 1024$ \\
\hline$[243]$ & Clarkson's Algorithm & Virtex-II-Pro FPGA & $4.2 \times 10^{-6}$ & $\mathrm{x}$ & $\mathrm{x}$ & $\mathrm{x}$ \\
\hline$[244]$ & Reverse Siegel LLL & Virtex-4 FPGA & $0.18 \times 10^{-6}$ & $\mathrm{x}$ & $\mathrm{x}$ & $\mathrm{x}$ \\
\hline$[244]$ & Reverse Siegel LLL & ASIC $130 \mathrm{nM}$ & $0.04 \times 10^{-6}$ & $\mathrm{x}$ & $\mathrm{x}$ & $\mathrm{x}$ \\
\hline$[250]$ & SB-LLL & ADRES & $0.17 \times 10^{-6}$ & $\mathrm{x}$ & $\mathrm{x}$ & $\mathrm{x}$ \\
\hline$[254]$ & Complex LLL & Virtex-5 FPGA & $0.79 \times 10^{-6}$ & $\mathrm{x}$ & $\mathrm{x}$ & $\mathrm{x}$ \\
\hline$[255]$ & Brun's Algorithm & ASIC $250 \mathrm{nM}$ & $0.07 \times 10^{-6}$ & $\mathrm{x}$ & $\mathrm{x}$ & $\mathrm{x}$ \\
\hline$[230]$ & CRAS-LLL & GTX690 GPU & $\mathrm{x}$ & $0.33 \times 10^{-6}$ & $1.37 \times 10^{-5}$ & $1.67 \times 10^{-2}$ \\
\hline$[232]$ & CRMB-LLL & GTX690 GPU + & $\mathrm{x}$ & $0.77 \times 10^{-6}$ & $1.30 \times 10^{-5}$ & $1.28 \times 10^{-2}$ \\
\hline
\end{tabular}

presented. The reconfigurable nature of GPUs allows gating the LR stage off when the user requirements are sufficiently guaranteed by the THP scheme, trading computational cost and performance. The LR stage has proven to be the main bottleneck in this parallel implementation.

In order to improve the LR stage in the implementation of precoding algorithms, several strategies for the parallel implementations of the LLL algorithm have been proposed. The CRAS-LLL algorithm allows carrying out different operations of the LLL algorithm simultaneously in parallel. In addition, the MB-LLL and CRMBLLL algorithms divide the original matrix into several submatrices, allowing to perform the CRAS-LLL algorithm on the submatrices. A heterogeneous platform based on a CPU and a GPU has been proposed for the implementation of these algorithms and its performance has been compared with implementations running on a GPU with DP capability and a multi-core architecture. Results show that the use of a GPU allows a speed-up ranging from 6 to 15 compared to multi-core CPUs. In addition, the block concept used in the CRMB-LLL allows achieving a $30 \%$ speed-up compared to the CRAS-LLL algorithm in the heterogeneous platform, which is the architecture that has achieved the best performance. 


\section{Part II}

\section{Limited Feedback}





\section{Chapter 6}

\section{Limited Feedback exploiting Frequency Correlation}

In FDD MU-MISO-OFDM systems, a large amount of channel information has to be provided by the MSs through the feedback link. In order to reduce this amount of information, the correlation in the frequency domain can be leveraged. In this chapter, two different feedback schemes based on vector quantization (VQ) [256] and on the KL [257] transform, respectively, are analyzed.

As seen in section 2.5, since CSIT cannot be obtained directly from the reverse link in FDD systems, it must be provided by the MSs through the feedback link. Usually, quantized feedback information is considered due to its better performance compared to unquantized feedback [144].

In MU-MISO-OFDM systems, the amount of feedback information can be considerable for a large number of OFDM subcarriers or a long CIR. Since the signal processing is performed on a per-subcarrier basis, information of all the subcarriers is required at the BS. In practice, CSI at the different subcarriers presents a high correlation that can be exploited to reduce the amount of feedback information.

First, this chapter presents an introduction to quantization. Next, two different strategies that allow taking advantage of the frequency correlation in order to improve the performance of the limited feedback scheme are presented. These strategies are based on the VQ and on the KL transform. 


\subsection{Introduction to quantization}

Given an input that usually can take any value in a continuous range, the purpose of quantization is to select a value from a finite set that best represents the input. Thus, the output can be represented by an integer $\{1,2, \ldots, N\}$ where $N$ is the size of the finite set. This operation is widely used in analog-to-digital conversion.

Depending on the dimensions of the input and the output, this process is known as scalar quantization or vector quantization. In scalar quantization, the input values as well as the elements of the finite set are scalars. In vector quantization, the quantizer operates with vectors instead of scalars. A detailed review on the history of quantization and different quantization techniques can be found in [258].

\subsubsection{Scalar quantization}

An $N$-level scalar quantizer can be defined by a mapping $\mathcal{Q}: \mathbb{R} \rightarrow \mathcal{C}$, where $\mathcal{C}$ is the codebook defined as

$$
\mathcal{C}=\left\{\hat{x}_{1}, \hat{x}_{2}, \ldots, \hat{x}_{N}\right\}, \hat{x}_{n} \in \mathbb{R} .
$$

The quantization rate or resolution is defined as $R=\log _{2} N$. Generally, $N$ is a power of 2 integer. If all the quantizer levels are assumed to have binary codewords of equal length, i.e. fixed rate quantizer, the number of bits per sample is also defined by $B=\log _{2} N$. Values $\hat{x}_{n}$ are commonly referred to as output values, quantized values or output levels. We assume that the indices of the output values are chosen so that

$$
\hat{x}_{1}<\hat{x}_{2}<\ldots<\hat{x}_{N}
$$

Associated with every output value, there is a region which is a partition of the real line such that

$$
\mathcal{R}_{n}=\left\{x \in \mathbb{R}: \mathcal{Q}(x)=\hat{x}_{n}\right\} .
$$

From this definition, it can be derived that the quantizer covers the whole range and the partition cells are non-overlapping:

$$
\begin{aligned}
\bigcup_{n} \mathcal{R}_{n} & =\mathbb{R}, \\
\mathcal{R}_{n} \bigcap \mathcal{R}_{n^{\prime}} & =\varnothing, \quad \text { for } n \neq n^{\prime} .
\end{aligned}
$$

A scalar quantizer is defined to be regular if each region $\mathcal{R}_{n}$ is an interval and

$$
\hat{x}_{n} \in\left(x_{n-1}, x_{n}\right) .
$$

The values $x_{n}$ are commonly referred to as boundary points, decision levels or decision thresholds [259]. Therefore, the regions can be defined as $\mathcal{R}_{n}=\left(x_{n-1}, x_{n}\right]$. 


\section{Conditions for optimality}

The design of the quantizer consists of obtaining the parameters of the quantizer, i.e. the output values and decision thresholds, that minimize the average distortion for a fixed $N$. In general, this problem does not have any explicit closed-form solution, but some effective algorithms can be used [259, Chap. 6].

There are two conditions for output levels and decision thresholds that are necessary for optimality by each one assuming that the other part is fixed:

\section{Optimal decision thresholds}

For a fixed set of output levels $\mathcal{C}=\left\{\hat{x}_{1}, \hat{x}_{2}, \ldots, \hat{x}_{N}\right\}$, each partition must satisfy

$$
\mathcal{R}_{n} \subset\left\{x: d\left(x, \hat{x}_{n}\right) \leq d\left(x, \hat{x}_{n^{\prime}}\right) ; \forall n^{\prime} \neq n\right\} .
$$

In other words, an input value $x$ is quantized to value $\hat{x}_{n}$ if and only if it produces the minimal distortion:

$$
\mathcal{Q}(x)=\hat{x}_{n} \Longleftrightarrow d\left(x, \hat{x}_{n}\right) \leq d\left(x, \hat{x}_{n^{\prime}}\right) ; \forall n^{\prime} \neq n .
$$

The most common measure of distortion is the squared error or squared Euclidean distance between the input and the output values, defined as

$$
d(x, \hat{x})=(x-\hat{x})^{2} .
$$

When using this distortion, (6.7) implies that for a given input $x$, the output $\hat{x}_{n}$ is chosen to minimize $\left|x-\hat{x}_{n}\right|$. In other words, the $n$th region consists of all the input values closer to $\hat{x}_{n}$ than to any other output value. This is achieved by selecting $x_{n}$ as the midpoint between the adjacent levels,

$$
x_{n}=\frac{\hat{x}_{n}+\hat{x}_{n+1}}{2} .
$$

For this reason, this condition is known as the nearest neighbor condition.

\section{Optimal output values}

For a given partition $\mathcal{R}_{n}$, its optimal output level $\hat{x}_{n}$ is the centroid or center of mass of that part of the input probability density function (PDF) that lies in the region $\mathcal{R}_{n}$. Considering the mean squared error, the optimal output value is given by

$$
\hat{x}_{n}=\mathbb{E}\left[x \mid x \in \mathcal{R}_{n}\right] .
$$

This condition is known as the centroid condition, and its fulfillment implies that the mean of the quantizer output is the same as the mean of the input, the quantizer output is uncorrelated with the quantizer error and the 
expected squared quantization error is the difference between the variances of the signal and the quantized output [259, Chap. 6]:

$$
\begin{aligned}
\mathbb{E}[\mathcal{Q}(x)] & =\mathbb{E}[x] \\
\mathbb{E}[\mathcal{Q}(x)(\mathcal{Q}(x)-x)] & =0 \\
\mathbb{E}\left[(x-\mathcal{Q}(x))^{2}\right] & =\mathbb{E}\left[x^{2}\right]-\mathbb{E}\left[(\mathcal{Q}(x))^{2}\right]
\end{aligned}
$$

In addition, the fulfillment of both conditions (optimal decision thresholds and optimal output values) considering the squared error distortion assures the regular condition expressed in (6.6).

\section{Uniform quantizer}

A uniform quantizer is defined as a regular quantizer where the decision thresholds are equally spaced and the output values are at the midpoints of the quantization intervals:

$$
\begin{array}{cc}
x_{n}-x_{n-1}=\Delta & n=2, \ldots, N-1 \\
\hat{x}_{n}=\frac{x_{n-1}+x_{n}}{2} & n=2, \ldots, N-1
\end{array}
$$

In case of bounded inputs, $x_{0}$ and $x_{N}$ are fixed by the boundaries of the inputs. However, in case of unbounded inputs, the quantizer has two overload regions where $x_{0}=-\infty$ and $x_{N}=\infty$. The output values of these regions are expressed as $\hat{x}_{1}=x_{1}-\Delta / 2$ and $\hat{x}_{N}=x_{n-1}+\Delta / 2$, as shown in Fig. 6.1.

The uniform quantizer provides a reasonably good performance for a wide variety of input signals, even though their PDFs are not uniformly distributed [259, Chap. 5]. In case of bounded inputs, the maximum possible error is $\Delta / 2$. The main problem of dealing with unbounded inputs is that the maximum error is infinite in the overload regions. However, most of the PDFs have a rapidly decreasing tail probability that reduces the probability of containing an input sample far from the output values for an appropriate quantizer design. For this reason, uniform quantizers are usually employed in analog-to-digital conversions.

\section{Non-uniform quantizer}

In non-uniform quantizers, the decision thresholds are not equally spaced, allowing to adapt the output levels to the shape of the input PDF. Thus, a better performance is obtained over the use of uniform quantizers. Non-uniform quantization can be performed using uniform quantizers together with non-linear functions at the input and output of the quantizer, known as compressor and expander functions. However, a general algorithm to obtain a non-uniform codebook from an 


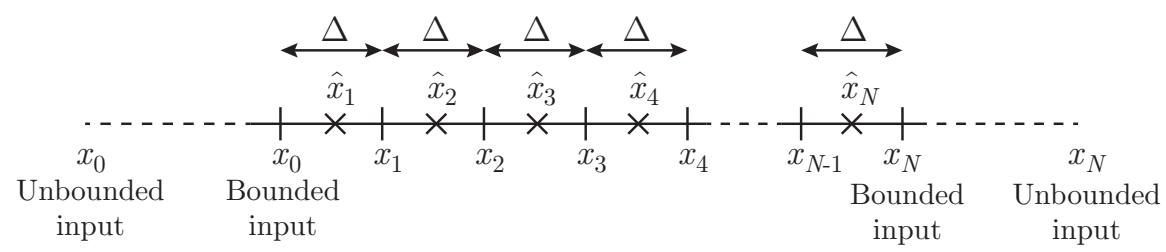

Figure 6.1: Example of uniform quantizer.

input $\mathrm{PDF}$ is presented and used in this thesis. In addition, this algorithm can also be applied when only training data is available instead of the PDF, although its details are not provided in this section.

Lloyd algorithm is an iterative algorithm based on the conditions of optimality. This algorithm was first described in an unpublished report in 1957 and published much later [260]. Independently, a similar algorithm was presented in [261], and therefore this algorithm is also known as Lloyd-Max algorithm.

The main idea behind Lloyd algorithm is to start with an initial codebook, a uniform codebook for instance, and iteratively improve the codebook by alternatively recalculating the decision thresholds and output values according to (6.10) and (6.11). The stopping criterion is usually based on the average distortion. If the reduction in the average distortion during the last iteration is below a threshold, convergence is assumed and the algorithm stops. In particular, the fractional drop in distortion

$$
\frac{D_{m}-D_{m+1}}{D_{m}}<\epsilon
$$

has shown to be an effective test to determinate the convergence [259, Chap. 6]. Given the PDF of the input signal, $f_{X}(x)$, the average distortion is obtained as

$$
D=\sum_{n=1}^{N} \int_{\mathcal{R}_{n}}\left(x-\hat{x}_{n}\right)^{2} f_{X}(x) \mathrm{d} x .
$$

The flow chart of the Lloyd algorithm is shown in Fig. 6.2. A different initial codebook could be used. However, a reasonable initial codebook according to the input PDF can considerably decrease the number of iterations. If the algorithm converges to a codebook in the sense that additional iterations do not change the codebook, then the achieved codebook satisfies both optimality conditions. 


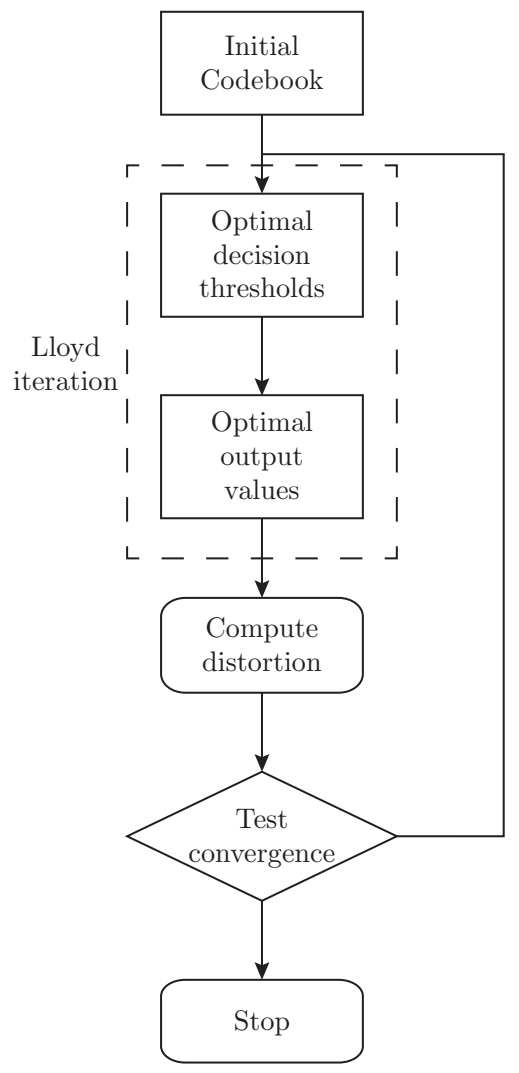

Figure 6.2: Lloyd algorithm for quantizer design.

\section{Bit allocation}

In communication systems, different parameters have to be simultaneously quantized with a limited number of bits. The manner in which those bits are distributed among the different parameters is known as bit allocation, and it is a major concern for obtaining the maximum benefit. In a system where the different parameters have the same "importance", an equal bit allocation can be used. However, bits have to be reasonably distributed in systems where the parameters have a different "importance".

\section{Real-valued bit allocation}

In order to perform the bit allocation, a trade-off between the distortion and the rate or quantization bits dedicated to quantize every random variable is necessary. Rate-distortion theory analyzes the problem of determining the minimal number of bits, $B$, allocated to the quantization of a random vari- 
able to achieve an expected distortion lower than $D[262,263]$. In the case of a Gaussian random variable with zero mean and variance $\sigma^{2}$, the ratedistortion function with squared error distortion is given by [28, Chap. 10]

$$
B(D)= \begin{cases}\frac{1}{2} \log _{2} \frac{\sigma^{2}}{D}, & 0 \leq D \leq \sigma^{2} \\ 0, & D>\sigma^{2}\end{cases}
$$

We can rewrite (6.19) to express the distortion in terms of the rate as

$$
D(B)=\sigma^{2} 2^{-2 B} .
$$

It is important to observe that this distortion is a lower bound. A performance close to this limit can be achieved by the joint quantization of multiple random variables. However, scalar quantization can also perform reasonably close to the rate-distortion function.

Assuming now that there exist $B$ bits to be allocated among the quantizers of a set of $K$ independent Gaussian random variables, $X_{1}, X_{2}, \ldots, X_{K}$, with zero mean and variances $\sigma_{1}^{2}, \sigma_{2}^{2}, \ldots, \sigma_{K}^{2}$. The definition of the squared error distortion is extended to

$$
d(\mathbf{x}, \hat{\mathbf{x}})=\|\mathbf{x}-\hat{\mathbf{x}}\|^{2}=\sum_{k=1}^{K}\left|x_{k}-\hat{x}_{k}\right|^{2},
$$

where $\mathbf{x}=\left[x_{1}, \ldots, x_{K}\right]$ and $\hat{\mathbf{x}}=\left[\hat{x}_{1}, \ldots, \hat{x}_{K}\right]$. Therefore, the optimal bit allocation that minimizes the average distortion subject to

$$
\begin{gathered}
B=\sum_{k=1}^{K} B_{k} \\
B_{k} \geq 0, \quad \forall k
\end{gathered}
$$

is given by [264]

$$
B_{k}=\max \left(\frac{1}{2} \log _{2}\left(\frac{\sigma_{k}^{2}}{\gamma}\right), 0\right),
$$

where $\gamma$ is the solution to

$$
\sum_{k=1}^{K} \max \left(\frac{1}{2} \log _{2}\left(\frac{\sigma_{k}^{2}}{\gamma}\right), 0\right)=B .
$$

The parameter $\gamma$ can be obtained through a kind of reverse water-filling [28, Chap. 10]. The achieved distortion is given by

$$
D=\sum_{k=1}^{K} D_{k}=\sum_{k=1}^{K} \min \left(\gamma, \sigma_{k}^{2}\right) .
$$




\section{Integer-valued bit allocation}

Although this bit allocation has considerable interest from a theoretical point of view, it presents some limitations in practical systems because the solution does not provide integer values of $B_{k}$. The extension to obtain integer-valued bit allocation is not straightforward, since it might lead to violate the restriction $\sum_{k=1}^{K} B_{k}=B$ or to require an excessive computation. For this reason, [259, Chap. 8] presents the greedy bit allocation (GBA) algorithm, a simple algorithm to obtain an integer-valued bit allocation. Although the algorithm is not optimal, it yields good assignments in practice.

The GBA algorithm makes use of the high resolution quantization approximation. This approximation allows expressing the distortion function for any input $\mathrm{PDF}$ as

$$
D(B) \approx a \sigma^{2} 2^{-2 B},
$$

where the constant $a$ can be calculated from the PDF of the normalized random variable $X / \sigma, f(x)$, as

$$
a=\frac{1}{12}\left(\int_{-\infty}^{\infty}(f(x))^{1 / 3} \mathrm{~d} x\right)^{3} .
$$

For a Gaussian random variable, the constant is $a_{\mathrm{g}}=\sqrt{3} \pi / 2$. This constant determines the distance from the rate-distortion bound shown in (6.20).

The GBA algorithm is shown in Algorithm 5. This algorithm essentially consists of allocating one bit at a time to the most needy quantizer, where the degree of neediness is measured by the average distortion given in (6.27). This algorithm together with the high resolution approximation allows to perform an integer-valued bit allocation among different quantizers with different PDFs.

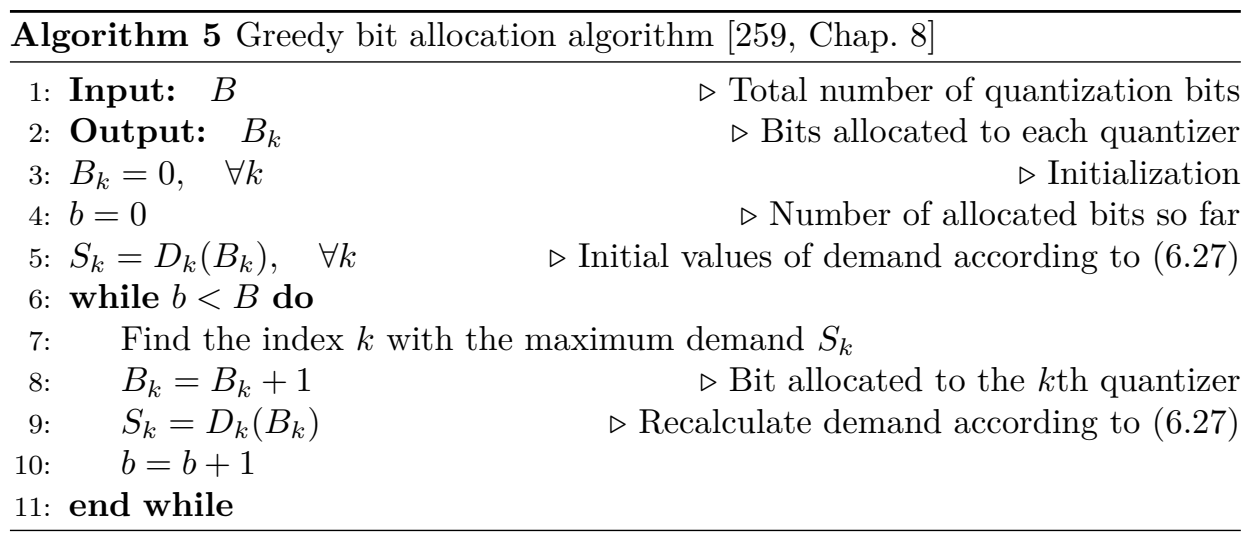




\subsubsection{Vector quantization}

VQ is a generalization of scalar quantization that deals with vectors instead of scalars. The availability of multiple dimensions introduces new ideas and techniques that offer a larger flexibility in the quantizer design. However, there are many similarities between vector and scalar quantization.

VQ has traditionally been used in lossy data compression due to the superior performance compared to the scalar quantization. However, the performance improvement comes at the cost of larger computational and memory requirements, which grow exponentially with the dimension [265].

A vector quantizer can by defined by a mapping $\mathcal{Q}: \mathbb{R}^{K} \rightarrow \mathcal{C}$, where $\mathcal{C}$ is the codebook defined as

$$
\mathcal{C}=\left\{\hat{\mathbf{x}}_{1}, \hat{\mathbf{x}}_{2}, \ldots, \hat{\mathbf{x}}_{N}\right\}, \hat{\mathbf{x}}_{n} \in \mathbb{R}^{K}
$$

The rate or resolution of the vector quantizer is defined as $R=\left(\log _{2} N\right) / K$, which expresses the number of quantization bits per vector component.

Associated with every output vector $\hat{\mathbf{x}}_{n}$, there is a region in $\mathbb{R}^{K}$ such that

$$
\mathcal{R}_{n}=\left\{\mathbf{x} \in \mathbb{R}^{K}: \mathcal{Q}(\mathbf{x})=\hat{\mathbf{x}}_{n}\right\} .
$$

From this definition, it can be determined that the quantizer covers the entire range and the partition cells are non-overlapping:

$$
\begin{aligned}
\bigcup_{n} \mathcal{R}_{n} & =\mathbb{R}^{K}, \\
\mathcal{R}_{n} \bigcap_{\mathcal{R}_{n^{\prime}}} & =\varnothing, \quad \text { for } n \neq n^{\prime} .
\end{aligned}
$$

Regions can be classified into granular and overload regions. A granular region has a finite $K$-dimensional volume, whereas an overload region is unbounded. A vector quantizer can be defined as regular if every region $\mathcal{R}_{n}$ is a convex set and

$$
\hat{\mathbf{x}}_{n} \in \mathcal{R}_{n}, \forall n \text {. }
$$

Figure 6.3 shows a comparison between scalar quantization and vector quantization for two independent, zero mean and unit variance Gaussian variables, $X_{1}$ and $X_{2}$. This case is equivalent to the quantization of a complex variable where the real and imaginary parts are uncorrelated. Figure $6.3 \mathrm{a}$ shows the different regions and output values corresponding to the scalar quantization of each variable, whereas Fig. 6.3b shows the regions and output values for a vector quantizer. These figures show that the vector quantizer has a larger flexibility since it can have different region shapes, which are adapted to the PDFs of the input random variables. 


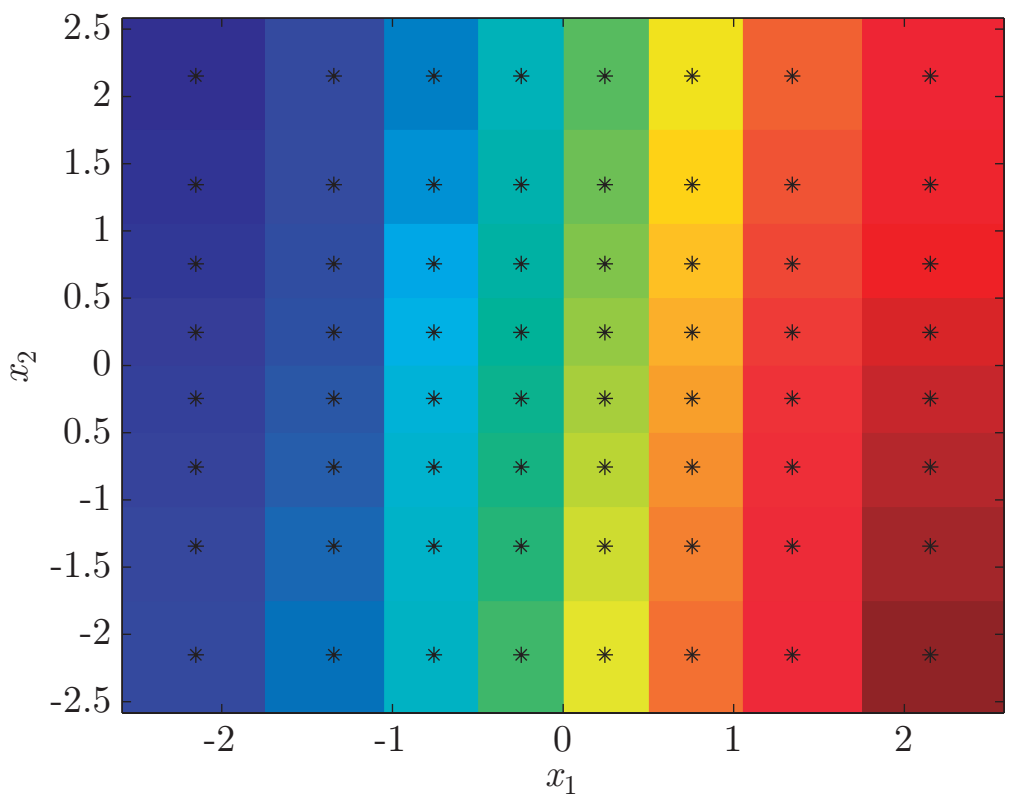

(a) Scalar quantization

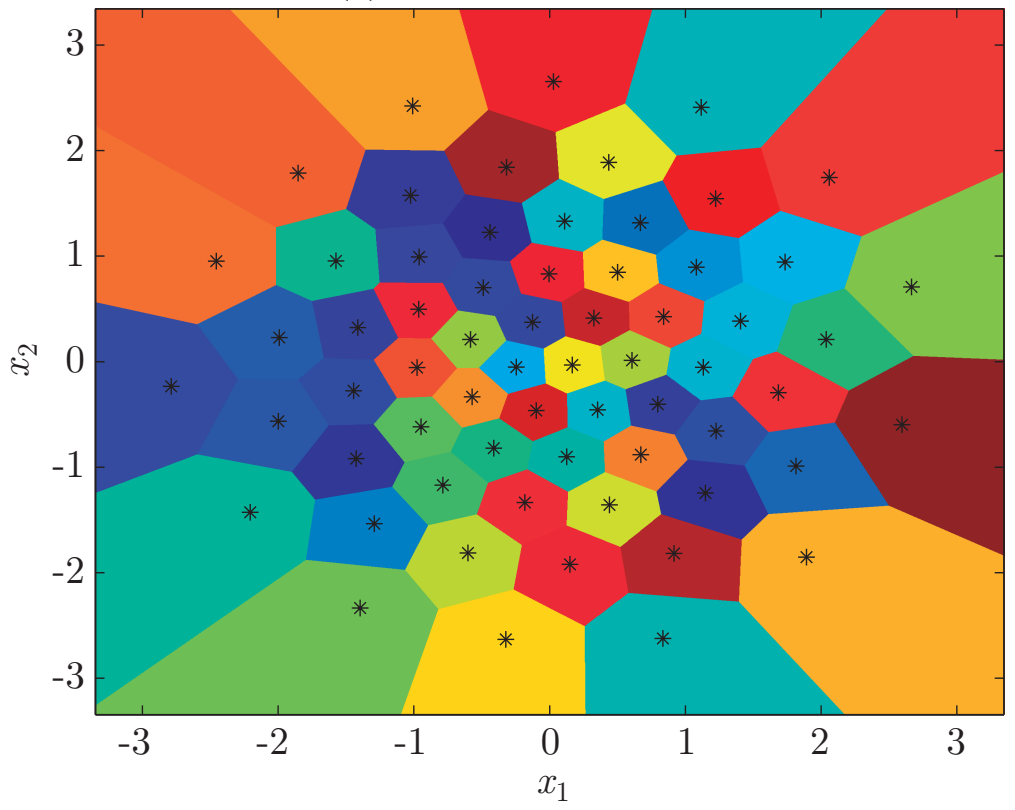

(b) Vector quantization

Figure 6.3: Scalar quantization vs. vector quantization for $X_{1}, X_{2} \sim N(0,1)$. 


\section{Conditions for optimality}

The optimality conditions for scalar quantization can be generalized to vector quantization [259, Chap. 11]. As in the scalar case, this thesis considers the squared error distortion applied over vectors defined in (6.21).

\section{Optimal decision thresholds}

For a fixed set of output levels $\mathcal{C}=\left\{\hat{\mathbf{x}}_{1}, \hat{\mathbf{x}}_{2}, \ldots, \hat{\mathbf{x}}_{N}\right\}$, each partition must satisfy

$$
\mathcal{R}_{n} \subset\left\{\mathbf{x}: d\left(\mathbf{x}, \hat{\mathbf{x}}_{n}\right) \leq d\left(\mathbf{x}, \hat{\mathbf{x}}_{n^{\prime}}\right) ; \forall n^{\prime} \neq n\right\} .
$$

In other words, an input value $\mathbf{x}$ is quantized to value $\hat{\mathbf{x}}_{n}$ if and only if it produces the minimal distortion:

$$
\mathcal{Q}(\mathbf{x})=\hat{\mathbf{x}}_{n} \Longleftrightarrow d\left(\mathbf{x}, \hat{\mathbf{x}}_{n}\right) \leq d\left(\mathbf{x}, \hat{\mathbf{x}}_{n^{\prime}}\right) ; \forall n^{\prime} \neq n .
$$

Thus, all input points closer to $\hat{\mathbf{x}}_{n}$ than to any other output vector must be assigned to regions $\mathcal{R}_{n}$. This condition is equivalent to the nearest neighbor condition for scalar quantizers.

\section{Optimal output values}

For a given partition $\mathcal{R}_{n}$, its optimal output level $\hat{\mathbf{x}}_{n}$ is the centroid or center of mass of that part of the input PDF that lies in the region $\mathcal{R}_{n}$ :

$$
\hat{\mathbf{x}}_{n}=\mathbb{E}\left[\mathbf{x} \mid \mathbf{x} \in \mathcal{R}_{n}\right]
$$

Again the fulfillment of the centroid condition implies that the mean of the quantizer output is the same as the mean of the input, the quantizer output is uncorrelated with the quantizer error and the expected squared quantization error is the difference between the variances of the signal and the quantized output [259, Chap. 11]:

$$
\begin{aligned}
\mathbb{E}[\mathcal{Q}(\mathbf{x})] & =\mathbb{E}[\mathbf{x}] \\
\mathbb{E}\left[\mathbf{x}^{T} \mathcal{Q}(\mathbf{x})\right] & =\mathbb{E}\left[\|\mathcal{Q}(\mathbf{x})\|^{2}\right] \\
\mathbb{E}\left[\|\mathcal{Q}(\mathbf{x})\|^{2}\right] & =\mathbb{E}\left[\|\mathbf{x}\|^{2}\right]-\mathbb{E}\left[\|\mathbf{x}-\mathcal{Q}(\mathbf{x})\|^{2}\right]
\end{aligned}
$$

In addition, the fulfillment of both conditions considering the squared error distortion assures the regular condition expressed in (6.33). 


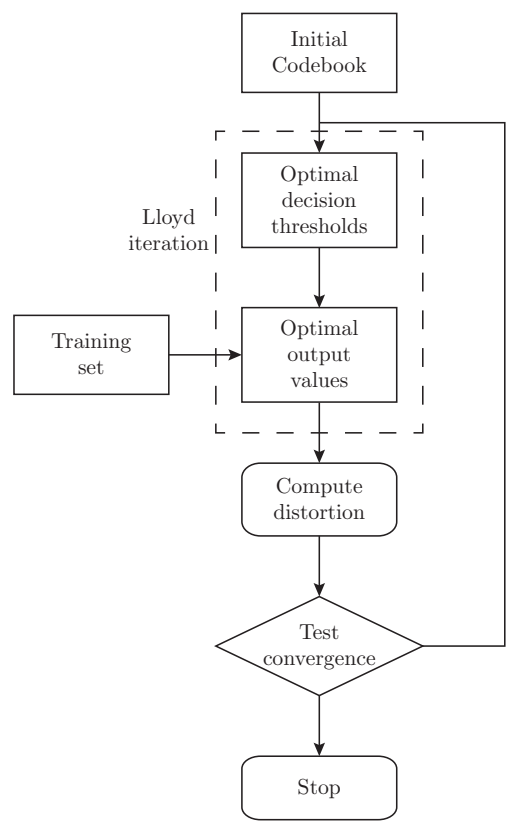

Figure 6.4: Lloyd algorithm for quantizer design with empirical data.

\section{Vector quantizer design}

Lloyd algorithm can be straightforwardly generalized to the vector quantization case by considering its optimality conditions. As previously seen in Fig. 6.2, this algorithm consists of starting with an initial codebook and iteratively improving it by alternatively applying the optimality conditions. Applied over vectors, this algorithm is known as the Generalized Lloyd algorithm [259, Chap. 11], k-mean algorithm [266] or LBG algorithm [267].

The optimality conditions expressed above are valid when the input PDF is known. In vector quantization, the centroid calculation may involve the evaluation of multiple integrals over non-regular regions in $\mathbb{R}^{K}$ which cannot be obtained through analytical methods. In addition, the input PDF is unknown in many practical systems. Therefore, the evaluation of the optimality conditions over empirical observations of the data is usually performed, as shown in Fig. 6.4.

Let $\mathcal{T}$ be a set of signal observations, a.k.a. training set,

$$
\mathcal{T}=\left\{\mathbf{v}_{1}, \ldots, \mathbf{v}_{T}\right\}
$$




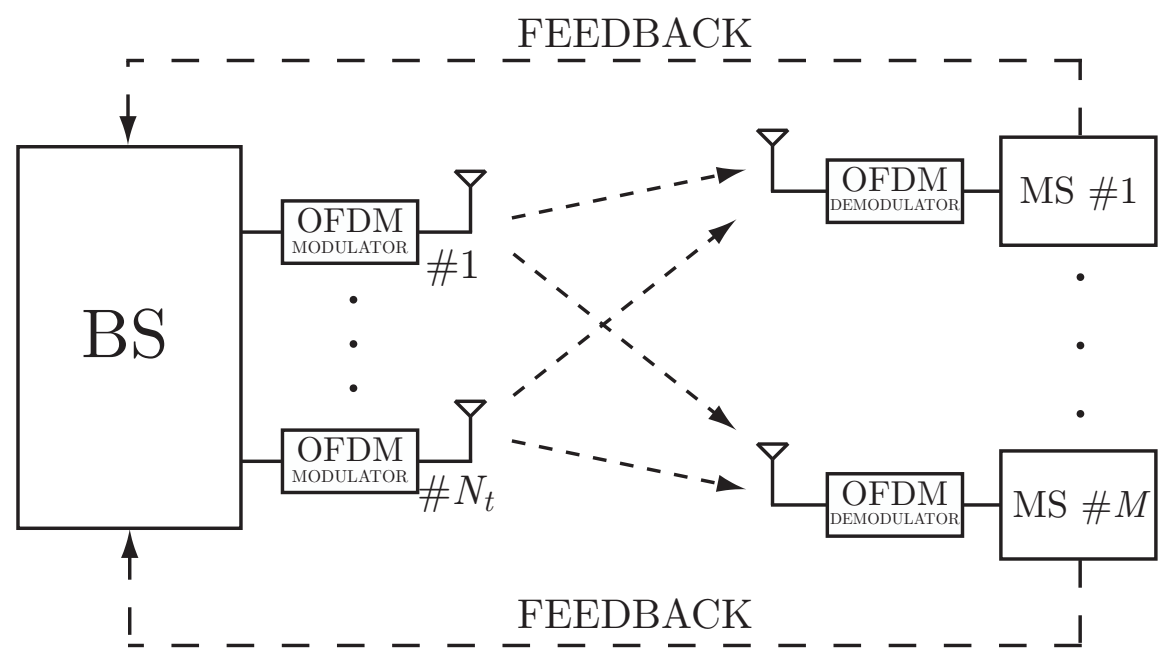

Figure 6.5: System model.

where $T$ is the size of the training set. The subset $\mathcal{T}_{n} \subset \mathcal{T}$ is defined as the subset that contains all the training vectors located in the region $\mathcal{R}_{n}$ :

$$
\mathcal{T}_{n}=\left\{\mathbf{v}_{t}: \mathbf{v}_{t} \in \mathcal{R}_{n}\right\} .
$$

Hence, the calculation of the centroid from the training set can be performed as

$$
\hat{\mathbf{x}}_{n}=\frac{1}{\left|\mathcal{T}_{n}\right|} \sum_{t=1}^{\left|\mathcal{T}_{n}\right|} \mathbf{v}_{t}
$$

The convergence of the Generalized Lloyd algorithm has been analyzed in [268]. Since the initial codebook may be decisive for the convergence of the algorithm, different techniques for the calculation of the initial codebook are proposed in [259, Chap. 11].

\subsection{Vector quantization over CFR pilots}

In this section, the performance of a feedback scheme based on VQ is evaluated in a MU-MISO-OFDM system (see Fig. 6.5). In the proposed scheme, the different MSs estimate the channel at the pilot subcarriers and group the estimations into vectors, which are quantized using VQ. Hence, the vector quantizer is used in the frequency domain [256]. 


\subsubsection{Channel estimation}

The channel estimation algorithm is performed as in [269]. This algorithm exploits the frequency correlation properties of the time varying wireless channel to estimate the different subcarriers. The estimation of the channel matrix is equivalent to estimating the $P$ frequency coefficients per transmit antenna for each of the $M$ MSs. Given the uncorrelated spatial fading assumption, the strategy consists of transmitting $N_{t}$ orthogonal pilot sequences, one from each transmit antenna, and estimating the gain parameter in each receive antenna. The SISO estimation algorithm is described and then, extended to the MISO case.

\section{SISO Channel Estimation}

The SISO channel estimation is based on transmitting pilot symbols in several equally spaced OFDM subcarriers, with data symbols in the rest of subcarriers. For a complex-valued, discrete-time OFDM channel with $L$ non-zero samples, at least $P \geq L$ pilot symbols are necessary to obtain an estimate of all $K$ subcarriers [269]. The SISO-OFDM channel estimation is performed as follows:

1. The frequency domain pilot symbols are collected into a $P \times P$ diagonal matrix:

$$
\mathbf{X}_{P}=\operatorname{diag}\{[x[0], x[K / P], \ldots, x[(P-1) K / P]]\} .
$$

2. The received symbols at the pilot subcarriers are collected in a vector:

$$
\mathbf{y}_{P}=[y[0], y[K / P], \ldots, y[(P-1) K / P]]^{T} .
$$

The least squares (LS) estimation of the pilot subcarriers is obtained by dividing the received symbols by the pilot transmitted symbols:

$$
\overline{\mathbf{h}}_{\mathrm{LS}}=\mathbf{X}_{P}^{-1} \mathbf{y}_{P},
$$

where $\overline{\mathbf{h}}_{\mathrm{LS}}=\left[\bar{h}_{\mathrm{CFR}}[0], \bar{h}_{\mathrm{CFR}}[K / P], \ldots, \bar{h}_{\mathrm{CFR}}[(P-1) K / P]\right]^{T}$ is a vector that contains the estimated CFR at the pilot subcarriers. The CIR is obtained by performing the $P$-point IDFT operation:

$$
\overline{\mathbf{h}}_{\mathrm{CIR}}=\frac{K}{P} \frac{1}{\sqrt{K}} \mathbf{F}_{P}^{H} \overline{\mathbf{h}}_{\mathrm{LS}}
$$

where $\mathbf{F}_{P}$ is the $P \times L$ matrix obtained by selecting the rows corresponding to the pilot subcarriers positions and the first $L$ columns of the $K \times K$ DFT matrix.

3. The estimated $K$-point CFR is obtained through the DFT with zero-padding of the estimated CIR,

$$
\overline{\mathbf{h}}_{\mathrm{CFR}}=\sqrt{K} \mathbf{F}_{L} \overline{\mathbf{h}}_{\mathrm{CIR}}=\frac{K}{P} \mathbf{F}_{L} \mathbf{F}_{P}^{H} \overline{\mathbf{h}}_{\mathrm{LS}},
$$


where $\mathbf{F}_{L}$ is the $K \times L$ matrix obtained selecting the first $L$ columns of the $K \times K$ DFT matrix.

\section{MIMO Channel Estimation}

In order to estimate the MU-MISO-OFDM channel, the SISO channel estimation is extended. Instead of transmitting $P$ pilot symbols spaced by $K / P$ subchannels, it can be seen as transmitting $P$ frequency domain sequences of length $N_{t}$. This scheme is shown in Fig. 6.6 where $p$ and $d$ generically denote a pilot symbol and a data symbol, respectively. Since the pilot symbols are orthogonal, the channel estimation can be performed independently for each transmit antenna at the different MSs. The total amount of pilot symbols necessary to estimate the MU-MISO-OFDM channel are $N_{t} P$.

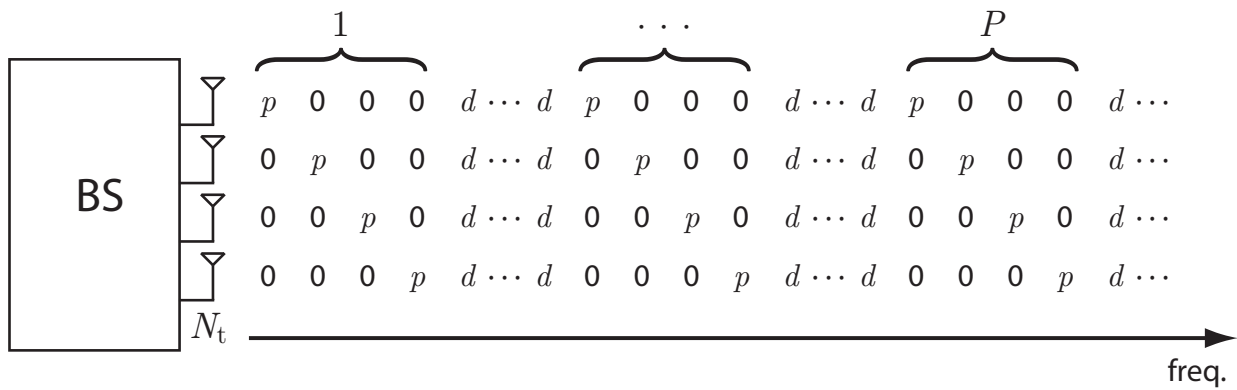

Figure 6.6: Pilot symbol distribution for channel estimation, where $p$ and $d$ generically denote a pilot symbol and a data symbol, respectively.

Choosing $P=K / N_{t}$, the best estimation of the channel matrix can be obtained at the cost of not transmitting data symbols during the estimation period. Thereby, pilot symbols in each antenna are spaced by $N_{t}$ subcarriers and all subcarriers are occupied by a pilot symbol during this period.

\subsubsection{Vector quantization}

Once the channel estimation has been performed, there are several techniques to feedback the channel information. Since channel estimation method is known at both the transmitter and receiver, an easy method consists of sending back the estimation of the channel at the position of the pilot subcarriers so that the interpolation can be performed at the BS.

Grouping the estimations at the position of the pilot subcarriers into one vector, $\mathbf{h}_{L S}$, and quantizing it would be the straightforward manner of using VQ. However, 
the large number of vector elements involve a large number of quantization bits, resulting in a prohibitive computational complexity of the codebook design and the searching process. This section proposes to divide the vector $\mathbf{h}_{L S}$ into smaller vectors of $L_{\mathrm{v}}$ components, such that

$$
\overline{\mathbf{h}}_{L S}^{l}=\left[\bar{h}_{L S}\left[l L_{\mathrm{v}}\right], \ldots, \bar{h}_{L S}\left[(l+1) L_{\mathrm{v}}-1\right]\right] \text {, for } l=1, \ldots, P / L_{\mathrm{v}}-1 .
$$

Then, VQ is performed over vectors $\overline{\mathbf{h}}_{L S}^{l}$. The accuracy of the CSIT will depend on the number of bits used to feed back these vectors. Given $B_{\mathrm{v}}$ feedback bits per vector of $L_{\mathrm{v}}$ components, a codebook with $2^{B_{\mathrm{v}}}$ codewords is built using the generalized Lloyd algorithm. The codebook can be expressed as

$$
\mathcal{C}=\left\{\mathbf{v}_{j}, 1 \leq j \leq 2^{B_{\mathrm{v}}}\right\}
$$

The codebook is generated from a random training set obtained from the statistical channel model. The same codebook can be equally generated at the BS and the different MSs by using the same seed to initialize the random generator. For small values of $B_{\mathrm{v}}$, the vector quantizer has a small number of codewords, therefore training and searching processes do not have a large computational cost. However, as $B_{\mathrm{v}}$ increases, the computational complexity of the designing and searching processes also increase.

Given a segment of the channel vector, $\overline{\mathbf{h}}_{L S}^{l}$, the MS selects the codeword which minimizes the squared Euclidian distance:

$$
\hat{\mathbf{v}}_{l}=\arg \min _{1 \leq j \leq 2^{B_{\mathbf{v}}}}\left\|\overline{\mathbf{h}}_{L S}^{l}-\mathbf{v}_{j}\right\|^{2} .
$$

Once these indices have been provided, the BS can recompose the entire channel from its own codebook given de index $l$ of $\hat{\mathbf{v}}_{l}$. The recomposed channel can be expressed as

$$
\hat{\mathbf{H}}[k]=\mathbf{H}[k]+\mathbf{E}[k],
$$

where $\hat{\mathbf{H}}[k]$ is the estimated channel at the BS, $\mathbf{H}[k]$ is the true channel and the $m$ th row in $\mathbf{E}[k]$ represents the additive estimation and quantization error vector for the $m$ th user and $k$ th subcarrier, whose entries are i.i.d. and follow a $\mathcal{C N}\left(0, \sigma_{e, k}^{2}\right)$ distribution. For a given subcarrier, the normalized mean square error (MSE) of the estimated channel is defined as

$$
\mathrm{MSE}=\frac{\mathbb{E}\left[\|\hat{\mathbf{H}}-\mathbf{H}\|_{\mathrm{F}}^{2}\right]}{\mathbb{E}\left[\|\mathbf{H}\|_{\mathrm{F}}^{2}\right]}=\frac{\mathbb{E}\left[\|\mathbf{E}\|_{\mathrm{F}}^{2}\right]}{\mathbb{E}\left[\|\mathbf{H}\|_{\mathrm{F}}^{2}\right]}
$$




\subsubsection{Numerical results}

In this section, the performance of the proposed limited feedback scheme in a MU-MISO-OFDM system is evaluated. The system consists of a BS with $N_{t}=4$ transmit antennas and $M=4$ single-antenna MSs. The transmission is done using $K=512$ subcarriers. The EPA and ETU from the Extended ITU channel models have been considered. The power delay profiles of these models can be found in Table 2.1. The spatial fading is assumed to be uncorrelated. The channel estimation is performed with $P=K / N_{t}=128$, such that there are no data symbol during this symbol period. Since the effect of the different SNRs in precoding algorithms has been previously studied, only the effect of having an imperfect knowledge of the matrix channel due to the limited feedback scheme is analyzed. Therefore, the noise is not considered either in the channel estimation or in the symbol detection, so errors at the MSs are only due to the imperfect CSIT.

Regarding the vector quantizer, several codebooks with different $L_{\mathrm{v}}$ and $B_{\mathrm{v}}$ have been obtained. Codebooks with $L_{\mathrm{v}}$ equal to $4,8,16$ and 32 have been calculated. The cost of each codebook is defined as $B_{\mathrm{v}} / L_{\mathrm{v}}$ and indicates the number of quantization bits per pilot subcarrier and MS. The values of $B_{\mathrm{v}}$ have been selected to obtain a comparable cost for different values of $L_{\mathrm{v}}$. However, obtaining codebooks for $B_{\mathrm{v}}>13$ has been unaffordable for computational restrictions. Therefore, the cost of the codebook is mainly determined by $L_{\mathrm{v}}$.

In Fig. 6.7, the MSE of the recomposed channel at the BS is plotted versus the cost of sending back the quantized version of the pilot subcarriers. It can be seen that the efficiency of the feedback information regarding the cost increases with $L_{\mathrm{v}}$. However, larger values of $B_{\mathrm{v}}$ are necessary in this case, affecting the complexity of the design of the codebook and also the complexity of the quantization process. It can also be noted that the MSE achieved with the EPA channel model is lower than the one achieved with the ETU channel model. This is mainly due to its larger coherence bandwidth, since VQ allows taking advantage of the correlation.

Figure 6.8 shows the BER obtained with the different precoding techniques versus the number of bits per vector $B_{\mathrm{v}}$ for $L_{\mathrm{v}}=16$. The value $L_{\mathrm{v}}=16$ has been chosen because it has shown a good efficiency and covers a wide range of MSE. As expected, the MSE decreases as the amount of feedback information increases. However, these figures are useful for selecting a precoding technique and the value of $B_{\mathrm{v}}$ for a given MSE requirement in the system. For example, the use of an LRbased precoding technique can save around 1-2 bits per each vector quantization compared to ZF or THP at the cost of a higher computational complexity. 


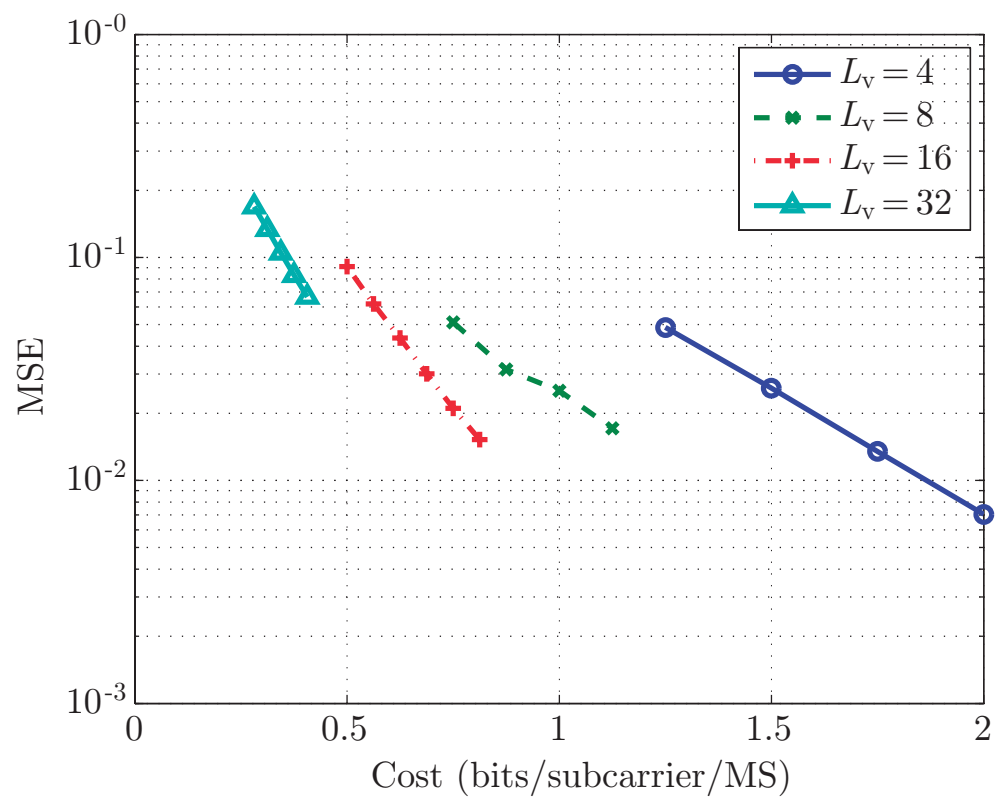

(a) EPA

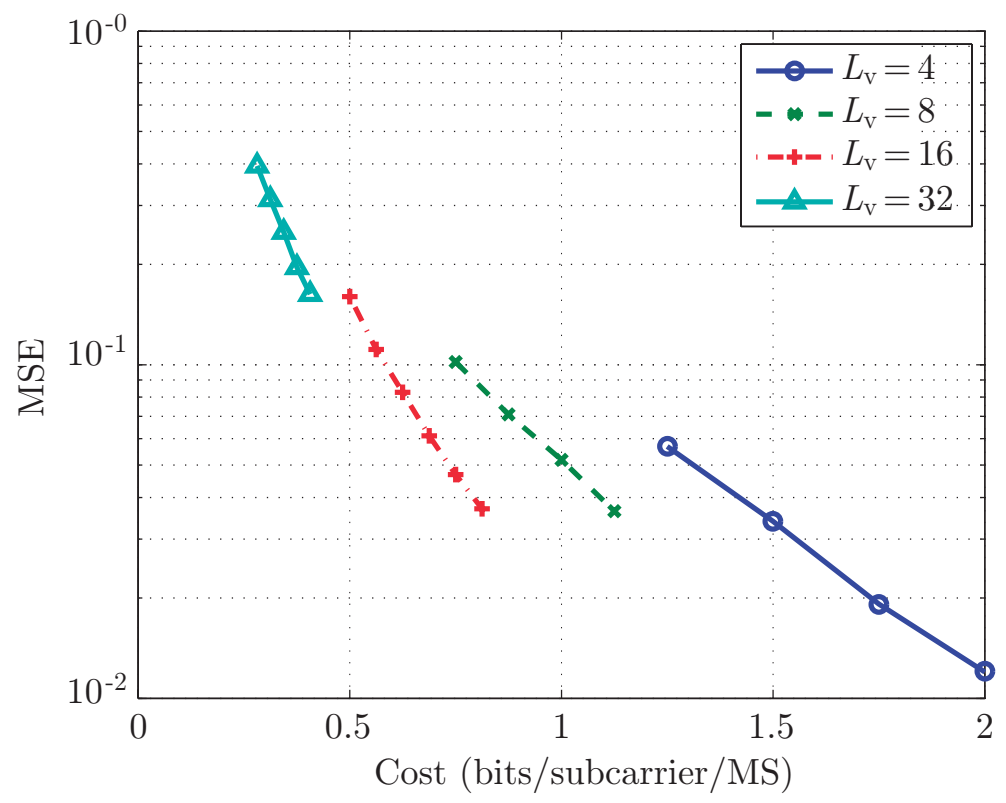

(b) ETU

Figure 6.7: Average MSE for the quantized channel matrices considering different $L_{\mathrm{v}}$ and $B_{\mathrm{v}}$. 


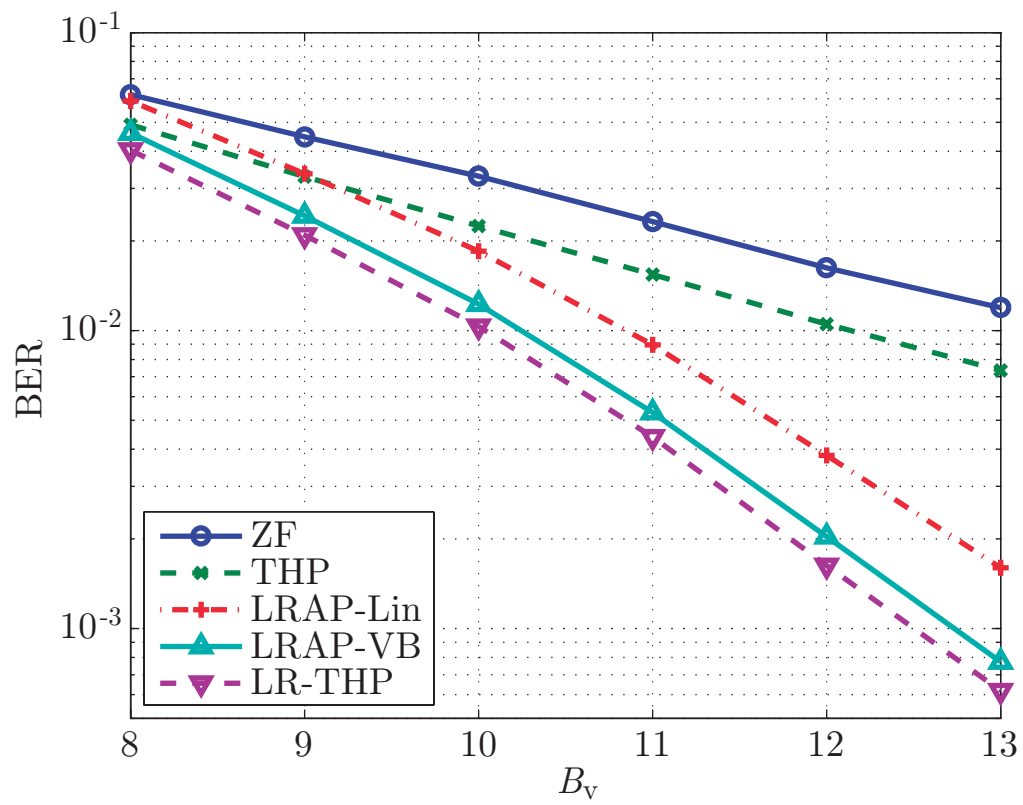

(a) EPA

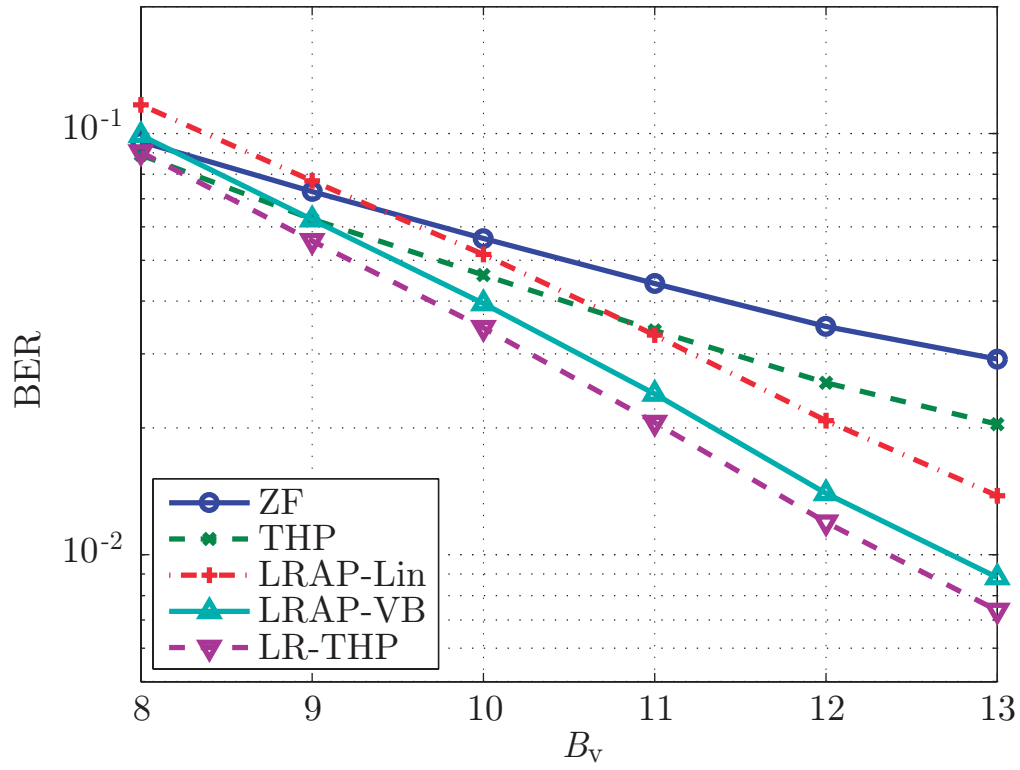

(b) ETU

Figure 6.8: BER for $L_{\mathrm{v}}=16$ and different values of $B_{\mathrm{v}}$. 


\subsubsection{Conclusion}

A limited feedback scheme based on VQ for MU-MISO-OFDM systems has been presented in this section. The CFR vector at the pilot positions is divided into segments of fixed length and quantized through VQ by a fixed number of bits. The performance achieved by different length and bits configurations has been compared. Simulations show that the higher efficiency for feedback information is achieved using longer vectors in the vector quantizer. However, it implies the use of larger codebooks, increasing the complexity of the codebook design and the quantization process.

In addition, the performance of different multiuser precoding algorithms has been evaluated when this channel quantization scheme has been used. Results show that nonlinear techniques based on LR are sufficiently robust against imperfect CSIT, outperforming the non-LR techniques. This main conclusion confirms the benefit of LR techniques even under imperfect CSIT.

\subsection{Channel quantization based on the Karhunen-Loève transform}

The previous section presented a VQ scheme that allowed to exploit the benefits of VQ and the correlation of the CFR. However, the design of the vector quantizer and the quantization process have a considerable computational cost for large codebooks. Because of that, other alternatives with a lower computational complexity can be found in the literature.

In [161], three different quantization schemes that make use of the frequency correlation to reduce the feedback information are compared: analog feedback, direction quantized feedback using VQ and time domain quantized feedback. The results show that the scheme based on time-domain quantization outperforms the other schemes in systems where all the subcarriers are modulated, requiring a lower computational complexity as well.

The KL transform has been used in many fields of engineering, especially in image and video compression [270]. The KL transform is considered an optimal orthogonal transform in the sense of decorrelation of Gaussian sources and energy compaction. This property makes the KL transform the optimal choice to quantize correlated Gaussian variables with a minimum MSE. In this section, a channel quantization scheme based on the KL transform is presented [257]. A comparison in terms of MSE and computational complexity between this scheme and the time-domain quantization scheme presented in [161] is shown. The comparison is carried out in a system where not all the subcarriers are modulated, as it usually occurs in modern communication standards [271]. 
The same system as in the previous section is again considered (see Fig. 6.5). For simplicity, the expressions of the channel in the time and frequency domain are repeated since they are used throughout this section. For a given transmit antenna and a given MS, the discrete CIR is given by $\mathbf{h}_{\mathrm{CIR}}=\left[h_{\mathrm{CIR}}[0], \ldots, h_{\mathrm{CIR}}[L-1]\right]^{T}$, where $L$ denotes the number of samples in the CIR. The CFR for the $K \geq L$ OFDM subcarriers is expressed as

$$
\mathbf{h}_{\mathrm{CFR}}=\sqrt{K} \mathbf{F}_{L} \mathbf{h}_{\mathrm{CIR}},
$$

where $\mathbf{h}_{\mathrm{CFR}}=\left[h_{\mathrm{CFR}}[0], \ldots, h_{\mathrm{CFR}}[K-1]\right]^{T}$ and $\mathbf{F}_{L}$ is an $K \times L$ matrix obtained by taking the first $L$ columns of a unitary $K \times K$ DFT matrix.

As in [161], it is assumed that channels are identically distributed and spatially uncorrelated for all MSs. However, in this case not all the subcarriers are used to carry data since only a subset of $K_{\mathrm{m}}$ subcarriers from a total number of $K$ subcarriers are modulated. The $\left(K-K_{\mathrm{m}}\right)$ unmodulated subcarriers, also known as virtual subcarriers or null subcarriers, are located at the edges of the used spectrum and used as a guard band. The CFR corresponding to the modulated subcarriers can then be expressed as

$$
\mathbf{h}_{\mathrm{CFR}, \mathrm{m}}=\mathbf{M h}_{\mathrm{CFR}},
$$

where $\mathbf{M}$ is a diagonal matrix with ones and zeros in the position of the modulated and unmodulated subcarriers, respectively.

\subsubsection{Time-domain quantization}

Time-domain channel quantization has been analyzed in [161] for the case where all the subcarriers are modulated. However, in this case, there are some unmodulated subcarriers at the edge of the baseband spectrum and the analysis is slightly different. It is important to note that, for the sake of clarity, the indices corresponding to the transmit antenna and the MSs have been omitted from the CIR and CFR, since the quantization has to be performed for every antenna and every MS independently.

The quantized CIR vector is given by

$$
\hat{\mathbf{h}}_{\mathrm{CIR}}=\mathcal{Q}\left\{\mathbf{h}_{\mathrm{CIR}}\right\} \text {. }
$$

Hence, the quantized CFR can be expressed as

$$
\hat{\mathbf{h}}_{\mathrm{CFR}}=\sqrt{K} \mathbf{F}_{L} \hat{\mathbf{h}}_{\mathrm{CIR}} \text {. }
$$

In order to compare the performance of different channel quantization schemes, the MSE of a quantized channel for the $K_{\mathrm{m}}$ modulated subcarriers is defined as

$$
\mathrm{MSE}=\mathbb{E}\left[\left\|\mathbf{h}_{\mathrm{CFR}, \mathrm{m}}-\hat{\mathbf{h}}_{\mathrm{CFR}, \mathrm{m}}\right\|^{2}\right] .
$$


Substituting (6.54) and (6.56) into (6.57), the MSE for the time-domain quantization scheme can be expressed as

$$
\mathrm{MSE}=K \mathbb{E}\left[\left(\mathbf{h}_{\mathrm{CIR}}-\hat{\mathbf{h}}_{\mathrm{CIR}}\right)^{H} \mathbf{F}_{L}^{H} \mathbf{M}^{H} \mathbf{M} \mathbf{F}_{L}\left(\mathbf{h}_{\mathrm{CIR}}-\hat{\mathbf{h}}_{\mathrm{CIR}}\right)\right] .
$$

When all the subcarriers are modulated, $\mathbf{M}$ becomes the identity matrix and (6.58) turns into $K \mathbb{E}\left[\left\|\mathbf{h}_{\mathrm{CIR}}-\hat{\mathbf{h}}_{\mathrm{CIR}}\right\|^{2}\right]$, as seen in [161], eq. (18). In fact, (6.58) shows that the MSE is minimized by minimizing the quantization error, $\mathbb{E}\left[\left\|\mathbf{h}_{\mathrm{CIR}}-\hat{\mathbf{h}}_{\mathrm{CIR}}\right\|^{2}\right]$. Assuming an accurate CIR estimation, it can be assumed that the error in the frequency-domain is equally distributed along the whole CFR (used and non-used subcarriers). Then, using the high resolution approximation of [259], the overall MSE in (6.58) can be approximated by

$$
\mathrm{MSE} \approx K_{\mathrm{m}} \mathbb{E}\left[\left\|\mathbf{h}_{\mathrm{CIR}}-\hat{\mathbf{h}}_{\mathrm{CIR}}\right\|^{2}\right] \approx K_{\mathrm{m}} a_{\mathrm{g}} \sum_{l=0}^{L-1} \sigma_{l}^{2} 2^{-b_{l}},
$$

where $\sigma_{l}^{2}$ is the variance of $h_{\mathrm{CIR}}[l]$, obtained from the covariance matrix $\mathbf{C}_{\mathbf{h}_{\mathrm{CIR}}}=$ $\mathbb{E}\left[\mathbf{h}_{\mathrm{CIR}} \mathbf{h}_{\mathrm{CIR}}{ }^{H}\right]$, and $a_{\mathrm{g}}=\sqrt{3} \pi / 2$ is the constant associated with the Gaussian $\mathrm{PDF}$. The parameter $b_{l}$ is the number of bits used to quantize $h_{\mathrm{CIR}}[l]$, and can be expressed as

$$
b_{l}=\max \left(\log _{2}\left(\frac{\sigma_{l}^{2}}{\gamma}\right), 0\right),
$$

where $\gamma$ is the solution to $\sum_{l}^{L} \max \left(\log _{2}\left(\sigma_{l}^{2} / \gamma\right), 0\right)=B$ and $B$ is the total number of quantization bits. It is important to note that this bit allocation process is equivalent to the one shown in section 6.1 .1 but applied over complex random variables. Taking a look at (6.59), it is interesting to point out that the MSE per subcarrier, i.e. MSE $/ K_{\mathrm{m}}$, is independent of the number of modulated subcarriers, $K_{\mathrm{m}}$.

\subsubsection{Karhunen-Loève-Domain Quantization}

Unlike the DFT where the orthogonal functions consist of sinusoids at different frequencies, the KL transform orthogonal functions are determined by the covariance matrix of the process. In this case, the covariance matrix of the modulated channel can be expressed as

$$
\mathbf{C}_{\mathbf{h}_{\mathrm{CFR}, \mathrm{m}}}=\mathbb{E}\left[\mathbf{h}_{\mathrm{CFR}, \mathrm{m}} \mathbf{h}_{\mathrm{CFR}, \mathrm{m}}^{H}\right]=\mathbf{M C}_{\mathbf{h}_{\mathrm{CFR}}} \mathbf{M}^{H},
$$

where $\mathbf{C}_{\mathbf{h}_{\mathrm{CFR}}}=\mathbb{E}\left[\mathbf{h}_{\mathrm{CFR}} \mathbf{h}_{\mathrm{CFR}}^{H}\right]=K \mathbf{F}_{L} \mathbf{C}_{\mathbf{h}_{\mathrm{CIR}}} \mathbf{F}_{L}^{H}$ is the covariance of the entire CFR.

The KL transformed channel is expressed as

$$
\mathbf{h}_{\mathrm{KL}}=\mathbf{V}^{H} \mathbf{h}_{\mathrm{CFR}, \mathrm{m}},
$$


where $\mathbf{V}^{H}$ is obtained from the eigendecomposition $\mathbf{C}_{\mathbf{h}_{\mathrm{CFR}, \mathrm{m}}} \mathbf{V}=\mathbf{V D}$, where the columns of matrix $\mathbf{V}$ are the eigenvectors of $\mathbf{C}_{\mathbf{h}_{\mathrm{CFR}, \mathrm{m}}}$ related to the corresponding eigenvalues of the diagonal matrix D. From (6.61) and assuming that the elements of $\mathbf{h}_{\mathrm{CIR}}$ are uncorrelated, it can be shown that the number of non-zero eigenvalues is related to the number of non-zero elements in the diagonal of $\mathbf{C}_{\mathbf{h}_{\mathrm{CIR}}}$. In addition, when all the subcarriers are modulated, i.e. $K_{\mathrm{m}}=K$ and $\mathbf{M}=\mathbf{I}$, the eigenvalues are equal to the diagonal of $\mathbf{C}_{\mathbf{h}_{\mathrm{CIR}}}$ since $\mathbf{F}_{L}$ forms a complete orthogonal basis of $\mathbf{C}_{\mathbf{h}_{\mathrm{CFR}, \mathrm{m}}}$.

The covariance matrix of the transformed channel is

$$
\mathbf{C}_{\mathbf{h}_{\mathrm{KL}}}=\mathbb{E}\left[\mathbf{h}_{\mathrm{KL}} \mathbf{h}_{\mathrm{KL}}^{H}\right]=\mathbf{V}^{H} \mathbf{C}_{\mathbf{h}_{\mathrm{CFR}, \mathrm{m}}} \mathbf{V}=\mathbf{D},
$$

that shows that the elements of $\mathbf{h}_{\mathrm{KL}}$ are uncorrelated and their variances are given by the eigenvalues of matrix $\mathbf{C}_{\mathbf{h}_{\mathrm{CFR}, \mathrm{m}}}$.

The channel at the BS can be calculated as

$$
\hat{\mathbf{h}}_{\mathrm{CFR}, \mathrm{m}}=\mathbf{V} \hat{\mathbf{h}}_{\mathrm{KL}}=\mathbf{V} \mathcal{Q}\left\{\mathbf{h}_{\mathrm{KL}}\right\} .
$$

Taking into account the recovered channel (6.64), the MSE for the KL-domain quantization scheme can be expressed as

$$
\begin{aligned}
\mathrm{MSE} & =\mathbb{E}\left[\left(\mathbf{h}_{\mathrm{KL}}-\hat{\mathbf{h}}_{\mathrm{KL}}\right)^{H} \mathbf{V}^{H} \mathbf{V}\left(\mathbf{h}_{\mathrm{KL}}-\hat{\mathbf{h}}_{\mathrm{KL}}\right)\right] \\
& =\mathbb{E}\left[\left\|\mathbf{h}_{\mathrm{KL}}-\hat{\mathbf{h}}_{\mathrm{KL}}\right\|^{2}\right] .
\end{aligned}
$$

As in the time-domain quantization scheme, equation (6.65) reveals that the MSE is due to the quantization stage. Using the high resolution approximation, the overall MSE can be approximated as

$$
\mathrm{MSE} \approx a_{\mathrm{g}} \sum_{l}^{L} \eta_{l}^{2} 2^{-b_{l}},
$$

where $a_{\mathrm{g}}=\sqrt{3} \pi / 2$ and $\eta_{l}^{2}$ is the variance of the elements of $\mathbf{h}_{\mathrm{KL}}$, obtained in (6.63). The parameter $b_{l}$ is the number of bits dedicated to quantize the $l$ th element of $\mathbf{h}_{\mathrm{KL}}$, which can be expressed as

$$
b_{l}=\max \left(\log _{2}\left(\eta_{l}^{2} / \gamma\right), 0\right),
$$

where $\gamma$ is the solution to $\sum_{l}^{L} \max \left(\log _{2}\left(\eta_{l}^{2} / \gamma\right), 0\right)=B$. The main advantage of this scheme compared to the time-domain quantization scheme is that, as the number of modulated subcarriers decreases, so does the variance of the elements to quantize, $\eta_{l}^{2}$. This fact can be proven using Cauchy's Interlace Theorem [272], taking into account that reducing the number of modulated subcarriers sets to zero the rows and columns of $\mathbf{C}_{\mathbf{H}_{\mathrm{m}}}$ corresponding to the unmodulated subcarriers. 


\subsubsection{Efficient eigendecomposition}

The computation of the eigenvalues and eigenvectors of the channel covariance matrix $\mathbf{C}_{\mathbf{h}_{\text {CFR,m }}}$ is usually the most computational demanding task in the KLdomain quantization procedure. The tridiagonalization of the matrix through Householder reflections, together with the QR method or the bisection method would efficiently obtain all the eigenvalues and eigenvectors. However, not all the eigenvalues and eigenvectors must be obtained since the number of non-zero eigenvalues is related to the number of paths in the channel, as seen in the previous section. In addition, the process can take advantage of the Hermitian Toeplitz structure of the channel covariance matrix in order to reduce the computational complexity. Under these conditions, iterative methods, such as Jacobi-Davidson or Lanczos, can be advantageous since they access the matrix only through matrixvector products in an iterative manner [273].

In the following scheme, the Lanczos method has been used because it has shown to have very good properties for parallel implementations on modern multi and many core processors [274]. In the Lanczos method, given an initial vector $\mathbf{r}$ which may be random or may contain some information about an eigenvector, an orthonormal basis $\mathbf{u}_{1}, \ldots, \mathbf{u}_{m}$ of the Krylov subspace $\mathcal{K}_{m}(\mathbf{A}, \mathbf{r})$ is established. In this basis, the matrix $\mathbf{A}$ is represented as a tridiagonal matrix [273]

$$
\tilde{\mathbf{A}}_{j}=\left[\begin{array}{cccc}
\alpha_{1} & \beta_{1} & & 0 \\
\beta_{1} & \alpha_{2} & \ddots & \\
& \ddots & \ddots & \beta_{j-1} \\
0 & & \beta_{j-1} & \alpha_{j}
\end{array}\right],
$$

where the calculation of the different $\alpha$ 's and $\beta$ 's are detailed in [273] and rewritten in Algorithm 6. This tridiagonal eigendecomposition can be performed in an efficient and parallelizable way with special techniques [275].

From Algorithm 6, it can be seen that the new basis is built by calculating one column at a time. The algorithm stops when a sufficiently large basis $\mathbf{U}_{j}$ has been obtained and, consequently, the eigenvalues of $\tilde{\mathbf{A}}_{j}$ (called Ritz values) provide good approximations to the eigenvalues of $\mathbf{A}$. The error, given by the norm of the residual vector, can be used as a convergence criteria and can be calculated from $\beta_{j}$ and $\mathbf{S}$ (see [273]). The Lanczos method first converges to the largest eigenvalues in terms of magnitude, although a spectral transformation (also known as shift-andinvert) can modify this behavior and even accelerate it [273]. Once the algorithm has converged, the approximated eigenvectors of $\mathbf{A}$ can be obtained as

$$
\mathbf{V}=\mathbf{U}_{j} \mathbf{S},
$$

where $\mathbf{U}_{j}=\left[\mathbf{u}_{1}, \ldots, \mathbf{u}_{j}\right]$. 


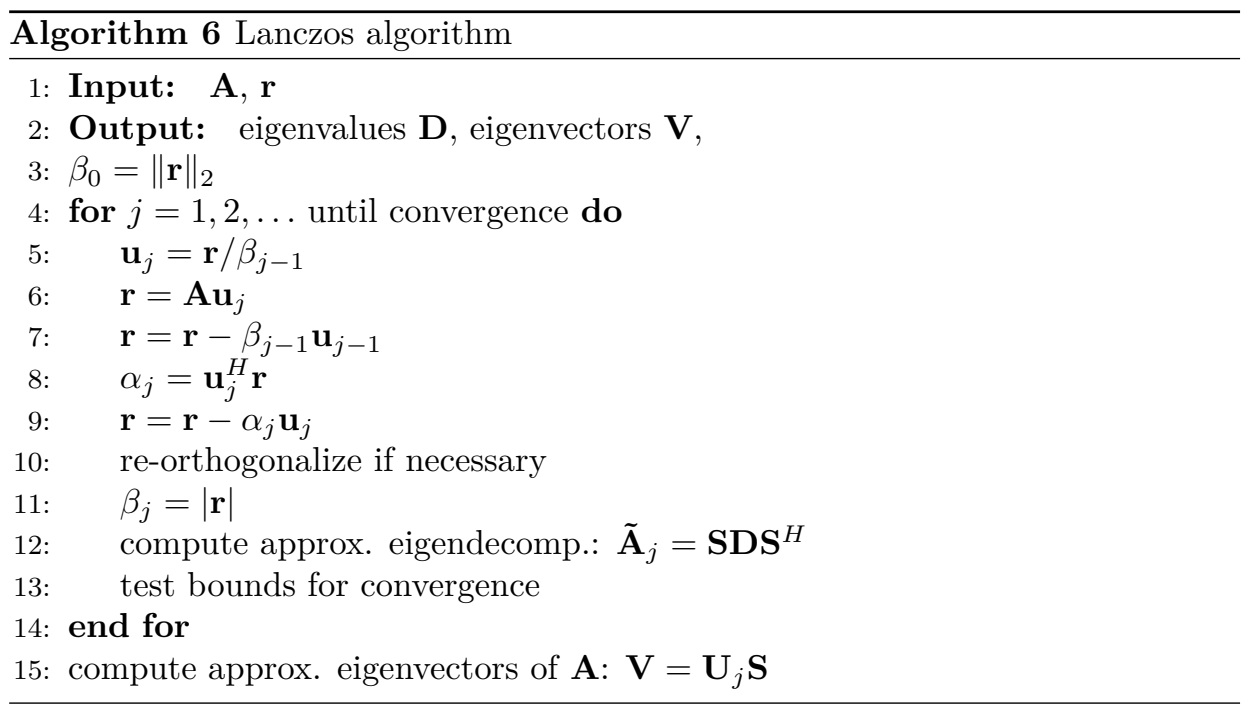

\subsubsection{Computational complexity}

This section compares the computational complexity of the time-domain and KL quantization schemes. The different operations and their computational cost are shown in Table 6.1. In the comparison, the quantization stage is omitted since it is common in both schemes, and the knowledge of $\mathbf{h}_{\mathrm{CIR}}$ and $\mathbf{h}_{\mathrm{CFR}, \mathrm{m}}$ is assumed.

Table 6.1: Comparison of the computational complexity

\begin{tabular}{lll}
\hline \hline \multicolumn{2}{c}{ Operations } & Computational complexity \\
TD scheme: & \\
MS $\quad \hat{\mathbf{h}}_{\mathrm{CIR}}=\mathcal{Q}\left\{\mathbf{h}_{\mathrm{CIR}}\right\}$ & \\
$\mathrm{BS} \quad \hat{\mathbf{h}}_{\mathrm{CFR}, \mathrm{m}}=\sqrt{K \mathbf{M F}} \hat{\mathbf{h}}_{\mathrm{CIR}}$ & $O\left(\min \left(L_{\mathrm{nz}} K_{\mathrm{m}}, K \log _{2} K\right)\right)$ \\
KL scheme: & \\
MS $\quad \mathbf{C}_{\mathbf{h}_{\mathrm{CFR}, \mathrm{m}}} \mathbf{V}=\mathbf{V D}$ & $O\left(L_{\mathrm{nz}} K_{\mathrm{m}}\left(\log _{2} K_{\mathrm{m}}+L_{\mathrm{nz}}\right)\right)$ \\
& $\mathbf{h}_{\mathrm{KL}}=\mathbf{V}^{H} \mathbf{h}_{\mathrm{CFR}, \mathrm{m}}$ & $O\left(L_{\mathrm{nz}} K_{\mathrm{m}}\right)$ \\
& $\hat{\mathbf{h}}_{\mathrm{KL}}=\mathcal{Q}\left\{\mathbf{h}_{\mathrm{KL}}\right\}$ & \\
$\mathrm{BS} \quad \hat{\mathbf{h}}_{\mathrm{CFR}, \mathrm{m}}=\mathbf{V} \hat{\mathbf{h}}_{\mathrm{KL}}$ & $O\left(L_{\mathrm{nz}} K_{\mathrm{m}}\right)$ \\
\hline \hline
\end{tabular}

It is important to note that $\hat{\mathbf{h}}_{\mathrm{CFR}, \mathrm{m}}$ in the time-domain scheme can be obtained through a matrix-vector product or FFT, depending on the value of $K, K_{\mathrm{m}}$ and the number of non-zero elements in the sampled CIR vector, $\mathbf{h}$, which has been denoted as $L_{\mathrm{nz}}$.

Regarding the KL scheme, it can be seen that the most computational expensive operation is the eigendecomposition. The computational complexity of the Lanczos 
algorithm is not straightforward to calculate since it is an iterative method and its convergence depends on the characteristics of the matrix [217, 273]. However, the covariance matrix $\mathbf{C}_{\mathbf{h}_{\mathrm{CFR}, \mathrm{m}}}$ only has $L_{\mathrm{nz}}$ non-zero eigenvalues and it has been checked through simulations that it converges in $L_{\mathrm{nz}}+1$ iterations for any value of $K_{\mathrm{m}}$ and an error lower than $10^{-10}$. The complexity of an iteration of the Lanczos algorithm is determined by the matrix-vector product of line 6 . Since matrix $\mathbf{A}$ has a Toeplitz structure, this operation can be carried out with a complexity of $O\left(K_{\mathrm{m}} \log K_{\mathrm{m}}\right)$ by using circulant matrices and the FFT [217]. Taking into account the final matrix product of line 15, the complexity of the Lanczos algorithm for the covariance matrices is $O\left(L_{\mathrm{nz}} K_{\mathrm{m}}\left(\log K_{\mathrm{m}}+L_{\mathrm{nz}}\right)\right)$, which is much lower than the complexity of an arbitrary dense matrix, $O\left(K_{\mathrm{m}}^{3}\right)$. It is important to point out that, except for this eigendecomposition, the computational complexity of the rest of the operations in both schemes is similar. Nevertheless, the eigendecomposition has to be carried out only when the statistics of the channel, i.e. $\mathbf{C}_{\mathbf{h}_{\mathrm{CIR}}}$, change.

\subsubsection{Numerical results}

In this section, numerical results are shown by comparing the performance of timedomain and KL quantization schemes. A system as the one presented in Fig. 6.5, with $M=N_{t}=4$, is considered. The physical layer parameters, such as $K=1024$ and the sampling frequency $f_{s}=15.36 \mathrm{MHz}$, are based on [271]. The EPA and the ETU models from the Extended ITU channels have been considered since they represent a low and a high delay spread channel, respectively. The delays and relative powers of the different taps can be found in Table 2.1. For the given $f_{s}, L_{\mathrm{nz}}=5$ and $L_{\mathrm{nz}}=9$ for EPA and ETU, respectively.

Figure 6.9 is provided as an illustrative example. In order to compare the quantization schemes, a perfect CIR estimation is assumed. It can be seen that, whereas the time-domain scheme quantizes the whole CFR, the KL scheme focuses on quantizing the modulated CFR. Thus, the KL scheme obtains a more accurate quantized channel in the band of interest.

The optimal bit allocation schemes proposed in (6.60) and (6.67) achieve a realvalued bit allocation (RBA) for the total number of quantization bits, $B$. However, for practical implementations where an integer-valued bit allocation is needed, the GBA detailed in Algorithm 5 is used. In the following figures, RBA denotes the theoretical results obtained in (6.59) and (6.66), whereas GBA denotes practical results for $10^{5}$ channel realizations using optimal non-uniform quantization for Gaussian sources.

Figure 6.10 shows the performance of the time-domain and KL quantization schemes in terms of the average MSE per modulated subcarrier, for a different number of subcarriers and a fixed number of quantization bits per transmit antenna, $B$, that depends on the delay spread of each channel. It can be shown that, whereas the 


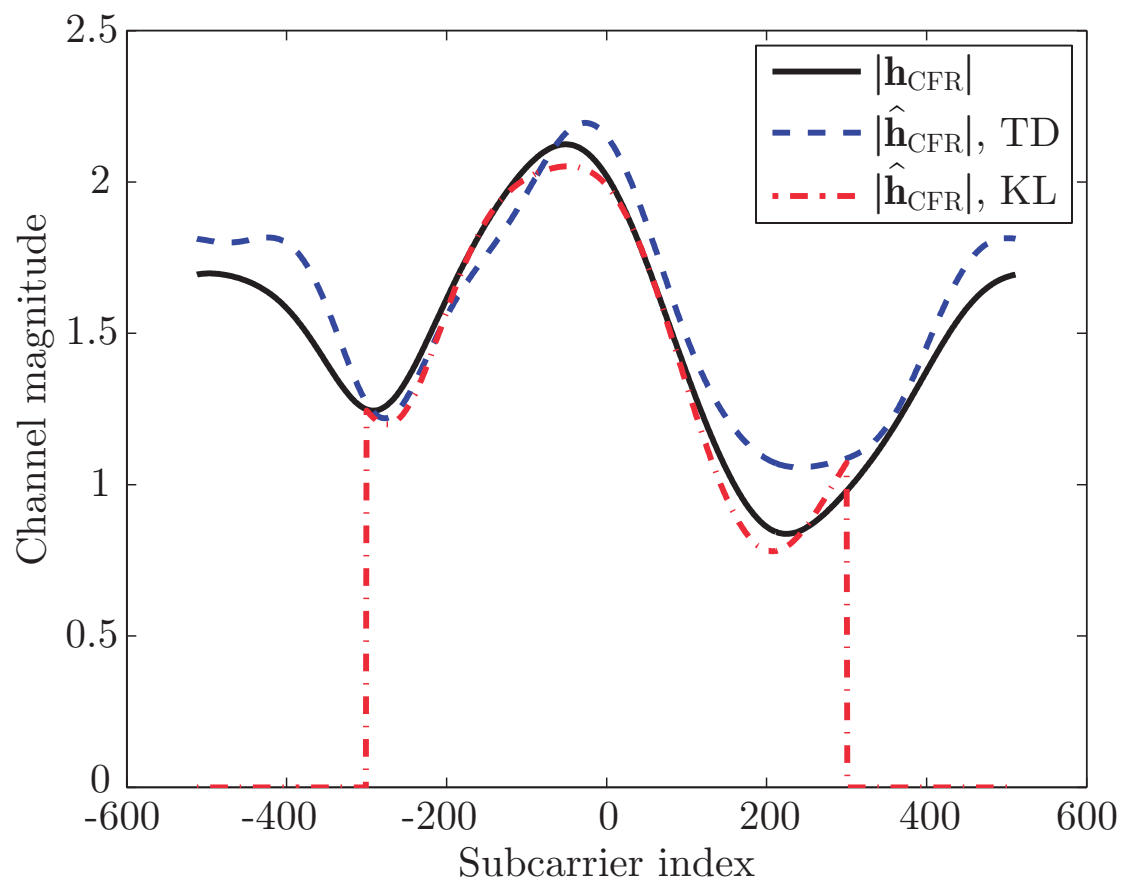

Figure 6.9: Quantized channel using time-domain (TD) and KL quantization schemes for EPA channel model with $K_{\mathrm{m}}=601$ modulated subcarriers using $B=30$ bits per transmit antenna.

MSE for the time-domain scheme remains constant with $K_{\mathrm{m}}$, the MSE for the KL scheme decreases with $K_{\mathrm{m}}$ due to the fact that the variance of $\mathbf{h}_{\mathrm{KL}}$ also decreases with $K_{\mathrm{m}}$. The irregularities in the MSE of the GBA implementations are due to the fact that, unlike the RBA scheme, the integer bit allocation can remain unchanged for small variations of the variance of the elements in $\mathbf{h}_{\mathrm{KL}}$.

Figure 6.11 shows the average MSE per modulated subcarrier of both quantization schemes for a different number of quantization bits and $K_{\mathrm{m}}=601$ modulated subcarriers [271]. In these figures, the channel estimation has been performed before the quantization stage, using 50 equally spaced pilot subcarriers. The SNR for the pilot symbols is $20 \mathrm{~dB}$. Whereas a linear interpolation of the pilot subcarriers would be sufficient in the KL scheme, a more complex channel estimation, such as the CIR channel estimation, is needed in the time-domain scheme. It can be noted that there is a gap of around 4 and 7 quantization bits between the time-domain and the KL schemes for a given value of MSE. This gap remains almost constant for low values of $B$. As the number of quantization bits increases, the quantization error decreases and becomes comparable to the estimation error, which is around $2 \cdot 10^{-3}$ and $7 \cdot 10^{-3}$ for EPA and ETU channels, respectively. Thus, the difference 


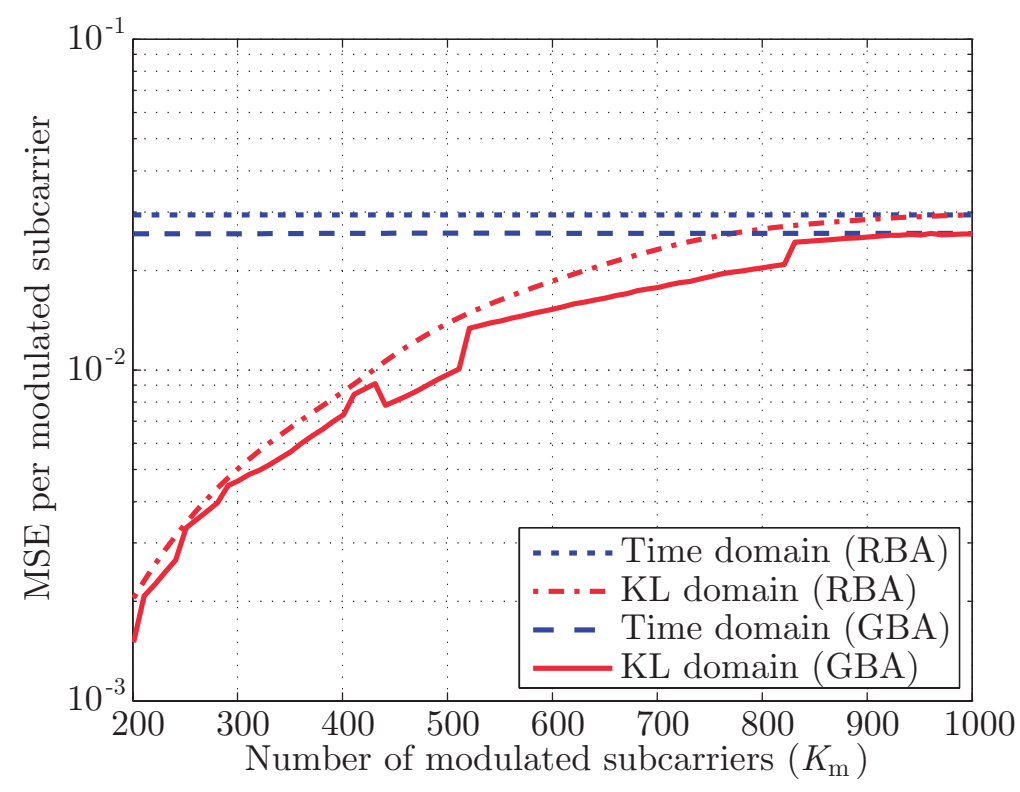

(a) EPA channel model, $B=30$ bits

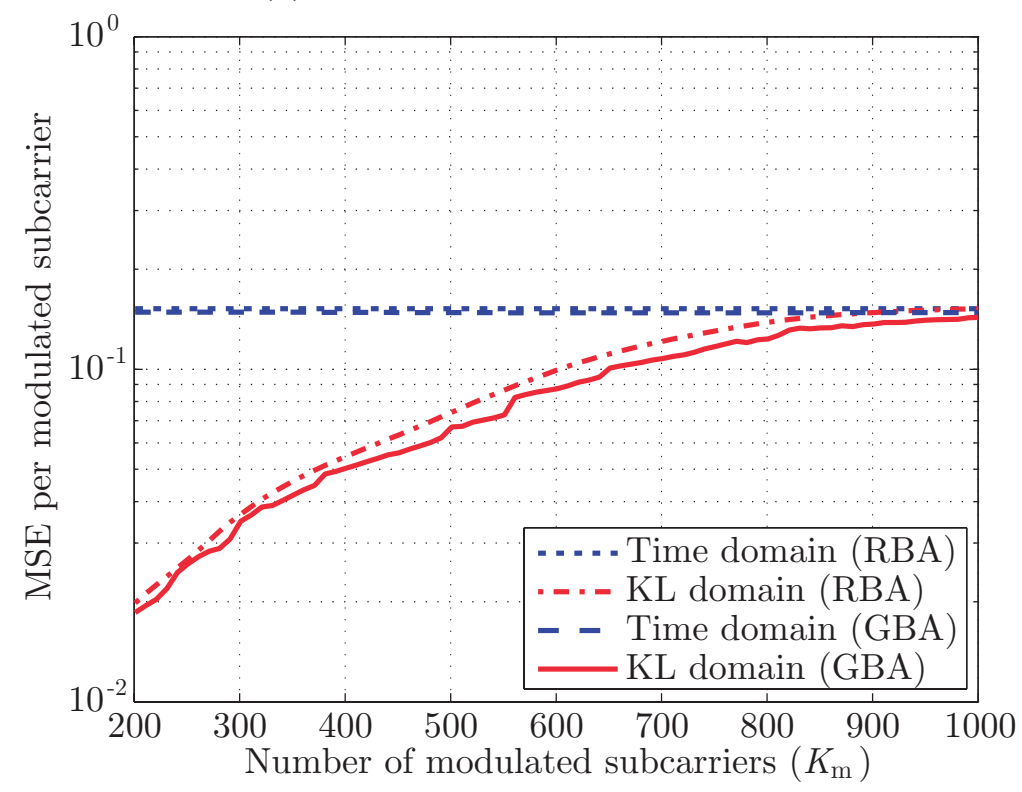

(b) ETU channel model, $B=60$ bits

Figure 6.10: MSE per modulated subcarrier for EPA and ETU channel models and different number of modulated subcarriers $K_{\mathrm{m}}$ using $B$ quantization bits per transmit antenna. 


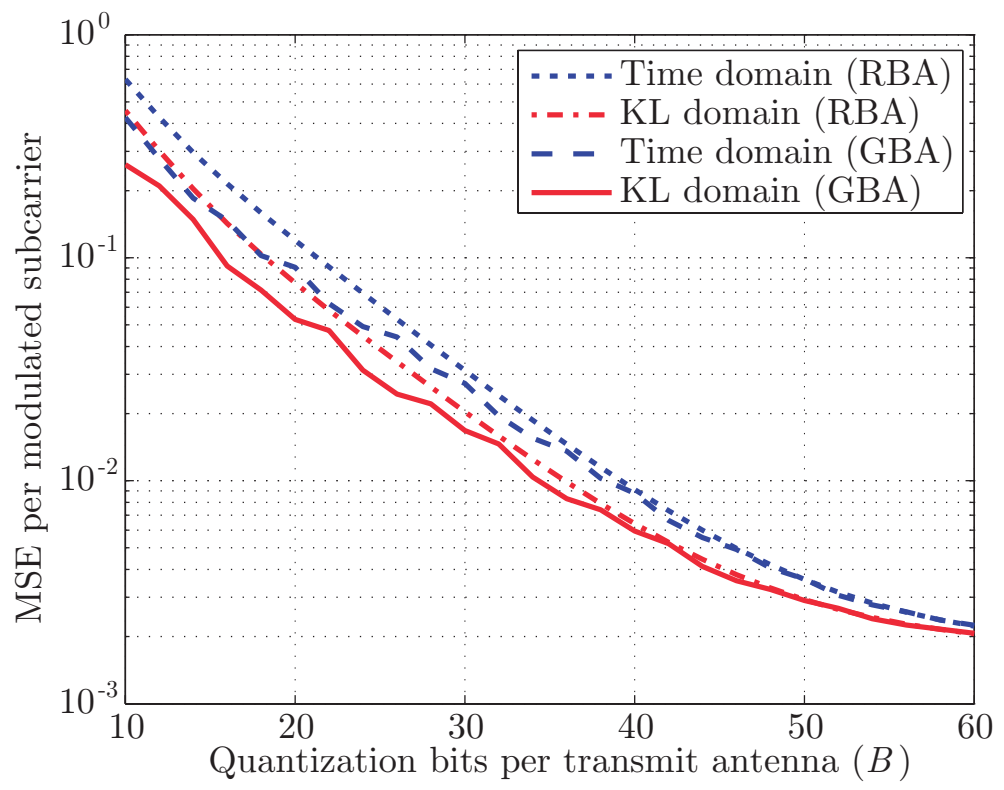

(a) EPA channel model

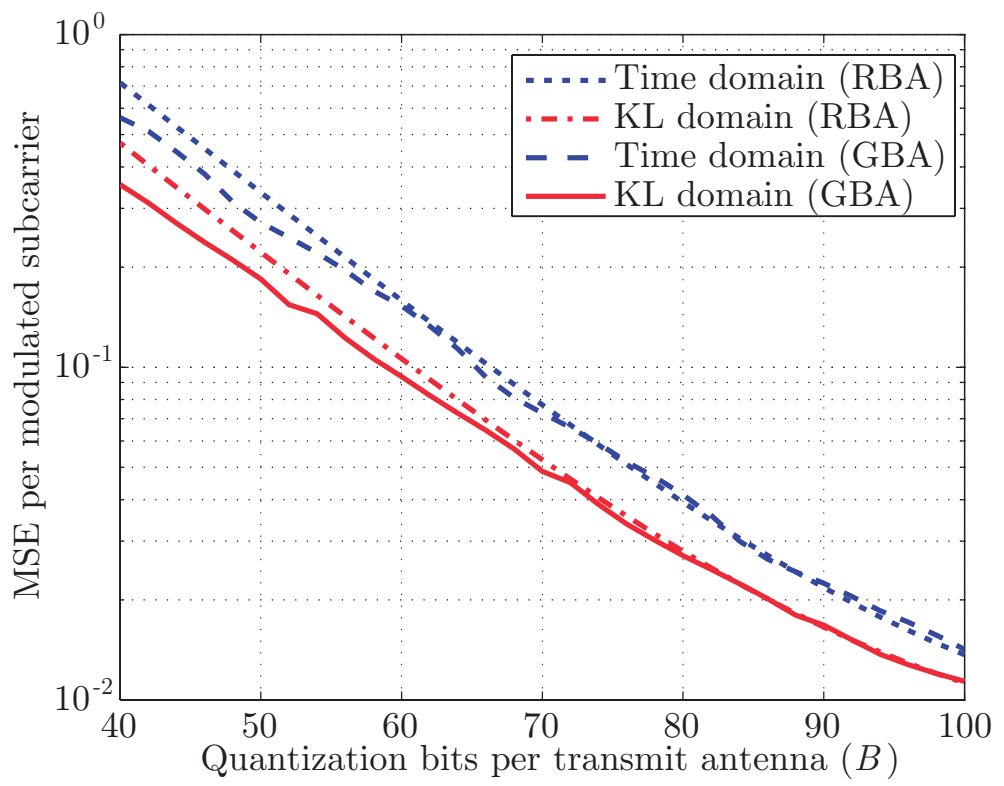

(b) ETU channel model

Figure 6.11: MSE per modulated subcarrier for EPA and ETU channel models and $K_{\mathrm{m}}=601$ modulated subcarriers and different number of quantization bits per transmit antenna. 
between the time-domain and KL schemes decreases. Figure 6.11 also validates the approximations used in (6.59) and (6.66).

\subsubsection{Conclusion}

This section has analyzed a channel quantization scheme based on the KL transform and has compared it with the time-domain channel quantization presented in [161] in a system where not all the subcarriers are modulated. In terms of complexity, the KL scheme has a slightly higher computational complexity than the time-domain scheme. The difference lies in the eigendecomposition that must be performed in the KL scheme, but only when the statistics of the channel change. On the other hand, the time-domain scheme requires a more complex channel estimation in order to obtain the CIR of the channel.

In terms of performance, theoretical MSE expressions have been obtained for both schemes. These expressions have been validated through simulations. Numerical results show that a reduction in the MSE of the quantized channel is achieved when using the KL scheme instead of the time-domain scheme. This reduction is higher as the number of unmodulated subcarriers increases.

\subsection{Conclusion}

This chapter has presented two feedback strategies to exploit the frequency correlation in MU-MISO-OFDM systems. The first strategy consists of grouping the estimations of the pilot subcarriers into vectors and quantizing them using VQ [256]. The performance of the system achieved with different VQ configurations has been evaluated. Results show that a higher feedback efficiency is obtained using longer vectors. However, the computational complexity of the quantization scheme increases with the length of those vectors, showing an interesting trade-off between performance and computational complexity. In addition, the different precoding techniques have been evaluated under imperfect CSIT achieved with the VQ feedback scheme. Results show that LR-based techniques can greatly deal with imperfect CSIT.

The second feedback strategy consists of using the KL transform to decorrelate the CFR before quantizing it [257]. Motivated by [161] where it is stated that time-domain channel quantization is the simplest and most effective technique to exploit the frequency correlation, both techniques are compared in a system with unmodulated subcarriers at the edges of the used spectrum. Results show that the proposed technique outperforms the time-domain channel quantization with a slightly higher computational complexity. 


\section{Chapter 7}

\section{Limited Feedback exploiting Spatial Correlation}

MIMO techniques allow leveraging the spatial dimension in addition to the time and frequency dimensions. With the use of this new dimension, the amount of CSIT increases with respect to SISO systems. However, the spatial correlation can be leveraged to reduce the CSIT. In this chapter, spatial statistical characterizations of the SCM and the Kronecker correlation model are presented. Based on these statistical characterizations, three different channel quantization schemes are proposed as limited feedback schemes and evaluated in a system considering the SCM [176, 220].

Limited feedback schemes presented in Chapter 6 took advantage of the frequency correlation in order to reduce the feedback information. Although these schemes are useful in MIMO systems, they have to be applied independently to the different SISO channels in a MIMO system, since the spatial dimension is not considered. Thus, they can be considered as extensions of SISO techniques applied to the MIMO case.

In this chapter, the correlation in the spatial dimension is analyzed and leveraged to reduce the feedback overhead. These schemes can be considered specific for MIMO systems, and not just extensions to the SISO case, since they make use of the spatial dimension. First, the spatial statistical characterization of the SCM is presented. Due to the high complexity and the large number of parameters involved in the SCM, the characterization is restricted to the high-correlation case. Second, a channel quantization scheme based on this statistical characterization is presented and evaluated in a coordinated system. 
Since the analysis of the SCM has shown to be a difficult task, the following section focuses on the Kronecker correlation model. This simpler model allows a deeper analysis of the spatial correlation. Statistical characterizations of the envelope and phase of the fading for each antenna have been stated assuming that the spatial correlation is known. Next, two different channel quantization schemes based on these statistical characterizations are presented and evaluated in a more realistic channel such as the SCM.

\subsection{Quantization based on the spatial characterization of the SCM}

This section proposes a low-complexity limited feedback scheme based on timedomain channel quantization for a cluster allowing joint processing (JP). JP is one of the techniques that falls under the umbrella of CoMP, and consists of several coordinated cells acting as a single and distributed antenna array. The limited feedback scheme leverages the spatial correlation between the different antennas of each BS without requiring any previous statistical knowledge of the channel. The reduction of feedback information is achieved by means of the quantization of the CIR coefficients. The contributions in this section can be summarized as follows:

- A proper pilot symbol allocation grid based on LTE-A that allows the pilot channel estimation in the cluster under consideration is proposed.

- A low-complexity limited feedback scheme based on channel quantization for highly correlated environments is presented. A practical expression of the quantization error has been obtained and compared to the error of the standard quantization scheme.

- The effect of imperfect CSIT due to the quantization schemes on some multiuser precoding techniques, such as ZF, THP and LR-THP, has been evaluated.

\subsubsection{System model}

The limited feedback scheme presented in this section exploits the spatial correlation of the transmit antennas in a BS covering a $120^{\circ}$ sector, as seen in Fig. 7.1. A highly correlated scenario and the SCM from the $3 \mathrm{GPP}$ have been considered. Without loss of generality, the BSs are equipped with a linear array of $N_{t}=4$ antennas in the examples and simulations, although it can be straightforwardly extended to other values. 


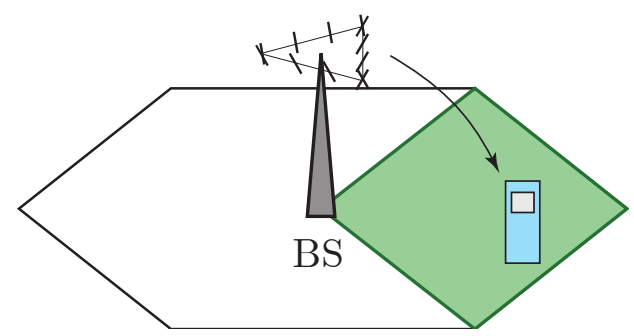

Figure 7.1: Example of a $120^{\circ}$ sector.

\subsubsection{Statistical characterization of the spatial channel}

The time-domain coefficients of the $S$-multipath channel between a single-antenna MS and the $n$th antenna of the BS are given by (2.19). To simplify the analysis, it is assumed that the antenna gain of the sector array is the same for the different subpaths, since the angular spread at the BS is only $2^{\circ}$ and $5^{\circ}$ for macrocell and microcell environments, respectively:

$$
G_{\mathrm{BS}, s, r}=G_{\mathrm{BS}}\left(\theta_{s, r, \mathrm{AoD}}\right)=G_{\mathrm{BS}}\left(\theta_{\mathrm{BS}}+\delta_{s, \mathrm{AoD}}\right)=G_{\mathrm{BS}, s},
$$

where $\theta_{\mathrm{BS}}$ and $\delta_{s, \mathrm{AoD}}$ were defined in Section 2 (see Fig. 2.9).

According to (2.19), the ratio between the coefficients of the $n$th and the $n^{\prime}$ th antennas in the same sector array for the $s$ th path can be expressed as:

$$
\frac{h_{n s}}{h_{n^{\prime} s}}=\frac{\sum_{r=1}^{R} \exp \left(j\left[k d_{n} \sin \left(\theta_{s, r, \mathrm{AoD}}\right)+\Phi_{s, r}\right]\right)}{\sum_{r=1}^{R} \exp \left(j\left[k d_{n^{\prime}} \sin \left(\theta_{s, r, \mathrm{AoD}}\right)+\Phi_{s, r}\right]\right)} .
$$

Note that the SCM assumes the same path loss and shadow fading for the channels of the antennas in the same sector array. Particularizing this expression to the case of $R=1$ subpaths as an example, the coefficients ratio becomes:

$$
\frac{h_{n s}}{h_{n^{\prime} s}}=\exp \left(j\left[k\left(d_{n}-d_{n^{\prime}}\right) \sin \left(\theta_{s, \mathrm{AoD}}\right)\right]\right), \quad n \neq n^{\prime} .
$$

Analyzing this expression, it can be observed that $\left|h_{n s} / h_{n^{\prime}}\right|=1$. Therefore, for the case of $R=1$, feedback information could be reduced since only the magnitude of one channel coefficient needs to be fed back. However, $R=20$ is the only value supported in the SCM specification [182]. Then, the magnitude of the ratio between channel coefficients $\left(\left|h_{n s} / h_{n^{\prime} s}\right|\right)$ in $(7.2)$ cannot be considered equal to 1. As stated previously, the correlation between $h_{n s}$ and $h_{n^{\prime} s}$ is due to the joint distribution of the AoA and the AoD, and it cannot be explicitly specified. However, it can be observed in Fig. 7.2 that for $R=20$, the ratio between these parameters can be approximated by a random variable following a Laplacian distribution centered in 1. Figure 7.2 shows the estimated PDF of the 


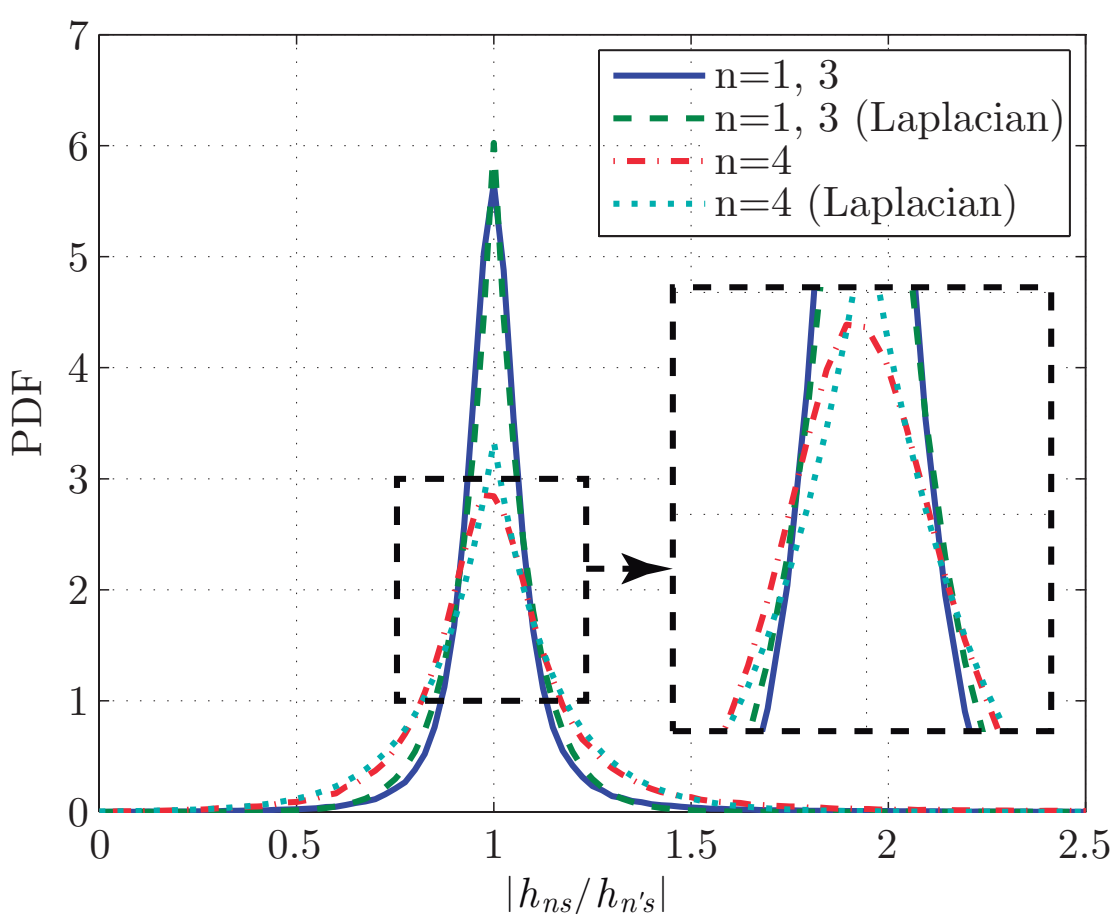

Figure 7.2: Estimated PDF of $\left|h_{n s} / h_{n^{\prime} s}\right|$ and its Laplacian approximaiton for $R=20$ subpaths and reference antenna $n^{\prime}=2$.

magnitude of the ratio between channel coefficients, $\left|h_{n s} / h_{n^{\prime}}\right|$, and its Laplacian approximation for the adjacent antennas $(n=1,3)$ and the further antenna $(n=$ 4), taking the antenna $n^{\prime}=2$ as the reference. Figure 7.2 and the rest of results in this section have been obtained using a Monte Carlo simulation considering a suburban macrocell scenario and an antenna spacing of $\lambda / 2[182,276]$. Note that the estimated PDF of the fourth antenna has a higher variance due to its lower correlation with the reference antenna.

The estimated PDFs of the different parameters of the channel coefficients (real and imaginary parts, angle and envelope) are shown in Fig. 7.3. For the sake of simplicity, the effect of path loss and shadow fading have not been taken into account in the statistical characterization since they are usually quantized and fed back separately. It can be seen that the real and imaginary parts of each channel coefficient show a Gaussian distribution centered in 0 and variance:

$$
\operatorname{var}\left(\mathfrak{R e}\left\{h_{n s}\right\}\right)=\operatorname{var}\left(\mathfrak{I m}\left\{h_{n s}\right\}\right)=\sigma_{\mathfrak{R e}-\mathfrak{I m}}^{2} \approx 0.085 .
$$

Component $\left|h_{n s}\right|$ is the magnitude of two normally distributed components with the same variance. Since the maximum variation of $\theta_{s, r, \mathrm{AoD}}$ in each sector is $120^{\circ}$, 


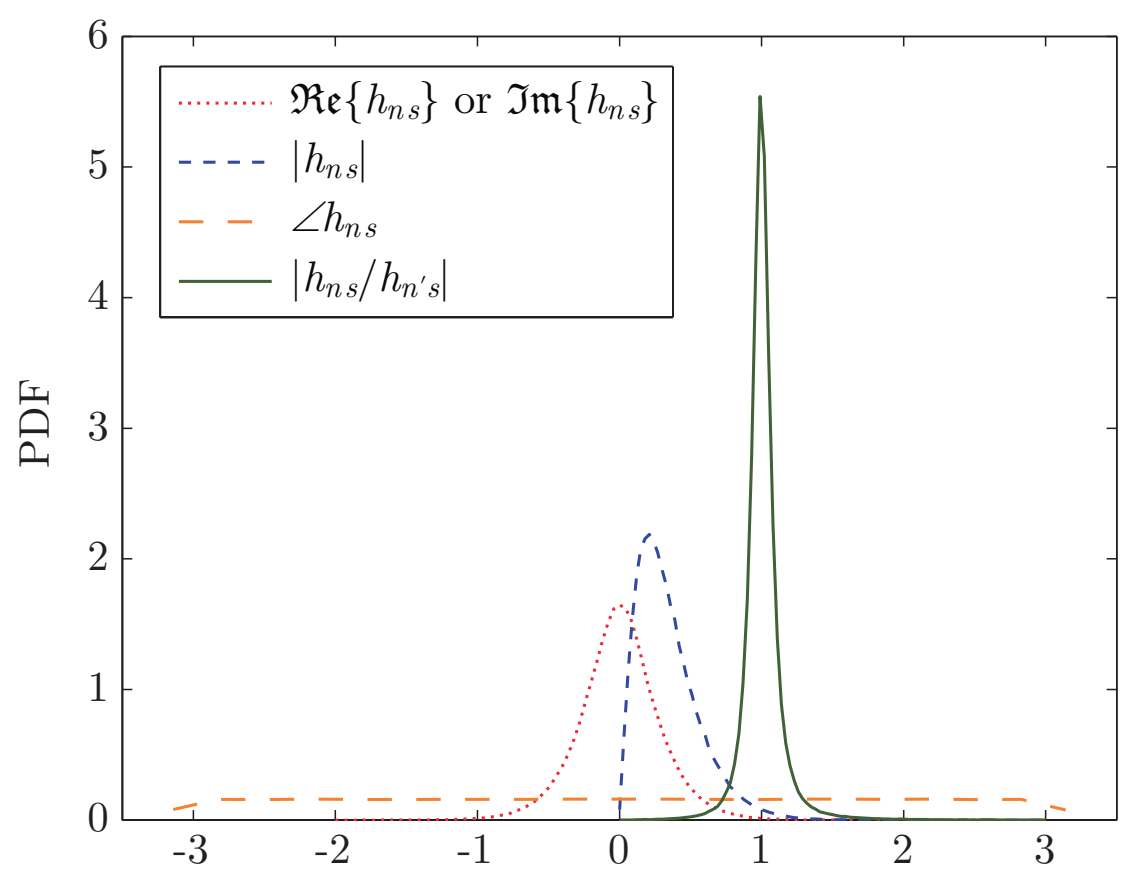

Figure 7.3: Estimated PDF of the different CIR parameters for $R=20$ subpaths, $n^{\prime}=2$ and $n=1,3,4$.

the real and imaginary parts are not completely uncorrelated. Thus, $\left|h_{n s}\right|$ does not present a strict Rayleigh distribution but it can be approximated as a Rayleigh distribution centered in $\sqrt{\frac{\pi}{2}} \sigma_{\mathfrak{R} \mathfrak{e}-\mathfrak{I m}} \approx 0.363$ and a variance:

$$
\operatorname{var}\left(\left|h_{n s}\right|\right) \approx 0.049
$$

The phase of the CIR coefficients shows a uniform distribution in $[-\pi, \pi)$, therefore its variance can be expressed as:

$$
\operatorname{var}\left(\angle\left(h_{n s}\right)\right)=\pi^{2} / 3 \approx 3.290 \text {. }
$$

Finally, the ratio between the magnitude of the channel coefficients, $\left|h_{n s} / h_{n^{\prime} s}\right|$, can be approximated by a random variable with Laplacian distribution centered in one and a variance given by

$$
\operatorname{var}\left(\left|h_{n s} / h_{n^{\prime} s}\right|\right) \approx 0.016
$$

which is the lowest variance among the analyzed parameters. 


\subsubsection{Proposed quantization scheme}

The proposed limited feedback scheme takes advantage of the reduced variance of the parameter $\left|h_{n s} / h_{n^{\prime} s}\right|$ in comparison to $\sigma_{\mathfrak{R e}-\mathfrak{I m}}^{2}$ due to the spatial correlation. In order to make use of the previous statistical analysis, the $n^{\prime}$ th antenna and the time-domain fading coefficient associated to this antenna are defined as references. The central antenna is the most suitable reference antenna in a linear array since the correlation between it and the rest of the antennas is the largest.

The steps of the scheme are summarized in Table 7.1. First of all, an optimal quantization using $B_{R}$ bits is performed over the real and imaginary parts of the reference time-domain fading, which shows a Gaussian PDF [261]. This step is shown in (7.1.A). It should be pointed out that, since $h_{n^{\prime} s}$ is the reference channel fading, a higher number of bits must be used to quantize it since the quantization error in this parameter will affect the rest of channel fading coefficients. Next, instead of quantizing the real and imaginary parts of the fading coefficients of the rest of the sector array, the parameters $\left|h_{n s} / \hat{h}_{n^{\prime} s}\right|$ and $\angle\left(h_{n s}\right)$ are properly quantized, as shown in (7.1.B) and (7.1.C). In these equations, $Q_{B_{M}}$ considers the quantization of a Laplacian random variable using $B_{M}$ bits [277], whereas $Q_{B_{P}}$ considers a uniform quantization with $B_{P}$ bits. The number of bits used to represent the quantized value $\hat{h}_{n s}^{\text {dif }}, B_{M}$, will be lower than the number of bits used to quantize $\mathfrak{R e}\left\{h_{n^{\prime} s}\right\}$ and $\mathfrak{I m}\left\{h_{n^{\prime} s}\right\}, B_{R}$, due to the significantly lower variance of $\left|h_{n s} / \hat{h}_{n^{\prime} s}\right|$. This scheme is referred to as differential quantization (DQ).

Table 7.1: DQ feedback scheme

$$
\begin{aligned}
& \text { A.- MS: channel quantization and feedback } \\
& \text { 1.- } \quad \hat{h}_{n^{\prime} s}=\mathcal{Q}_{B_{R}}\left(\mathfrak{R e}\left\{h_{n^{\prime} s}\right\}\right)+j \mathcal{Q}_{B_{R}}\left(\mathfrak{I m}\left\{h_{n^{\prime} s}\right\}\right) \\
& \text { 2.- } \quad \hat{h}_{n s}^{\text {dif }}=\mathcal{Q}_{B_{M}}\left(\left|h_{n s} / \hat{h}_{n^{\prime} s}\right|\right) \\
& \quad \quad \hat{\gamma}=\mathcal{Q}_{B_{P}}\left(\angle\left(h_{n s}\right)\right) \\
& \text { B.- BS: channel reconstruction } \\
& \text { 1.- } \quad \hat{h}_{n s}=\hat{h}_{n s}^{\text {dif }}\left|\hat{h}_{n^{\prime} s}\right| \exp (j \hat{\gamma}) \\
& \hline
\end{aligned}
$$

It has to be pointed out that, in order to reduce the quantization error, $\hat{h}_{n s}^{\text {dif }}$ is obtained from the quantized version of $h_{n^{\prime}}$, that is, $\hat{h}_{n^{\prime}}$. Thus, the BS can reconstruct the parameter with lower quantization error. On the other hand, the variable $\angle\left(h_{n s}\right)$ presents a uniform distribution in $[-\pi, \pi)$, hence it is quantized through uniform quantization. Quantizing $\angle\left(h_{n s} / h_{n^{\prime} s}\right)$ instead of $\angle\left(h_{n s}\right)$ does not offer any benefit since both variances are similar. Therefore, additional mathematical operations can be avoided by quantizing $\angle\left(h_{n s}\right)$ directly as in (7.1.C). The reconstructed coefficients at the BS once $\hat{h}_{n s}^{\text {dif }}$ and $\hat{\gamma}$ have been received are expressed in (7.1.D). 
Finally, in order to improve the stability of $\left|h_{n s} / \hat{h}_{n^{\prime} s}\right|$ quantization, the MS can choose the reference antenna as the central one $\left(n^{\prime}=2,3\right)$ showing a larger $\left|\hat{h}_{n^{\prime} s}\right|$, using only one additional bit for sending back this information.

\subsubsection{Extension to CoMP}

Since the limited feedback scheme presented in Table 7.1 reduces the feedback overhead related to the antennas in a sector array, it could be independently applied to the different BSs of a coordinated cluster such as the one shown in Fig. 7.4. However, it is necessary to extend the LTE-A RSs pattern in order to allocate enough pilot symbols to perform the composite channel estimation.

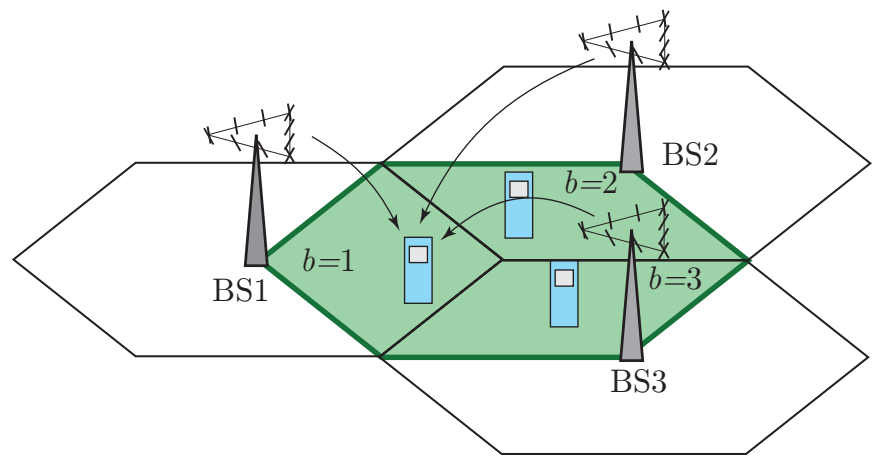

Figure 7.4: Example of a coordinated cluster of $|\mathcal{B}|=3$ cooperating sectors and $N_{t}=4$ transmit antennas per sector.

The LTE slot, also used in LTE-A, is composed of 7 OFDM symbols with a duration of $0.5 \mathrm{~ms}$, whereas the LTE subframe consists of two LTE slots. The number of subcarriers in an OFDM symbol is represented by $K$. However, not all the subcarriers are modulated. Only $K_{\mathrm{m}}$ subcarriers placed around the zero frequency in the baseband spectrum are modulated. The subcarrier spacing is $\Delta f=15 \mathrm{kHz}$ and it remains constant for the different bandwidth configurations. The sampling frequency $f_{s}$ is proportional to $K$. In LTE-A, CSI-RSs have been introduced for the use of up to 8 transmit antennas. However, for backward compatibility, the CSI-RSs must be placed in resource elements (REs) that do not contain cell-specific reference signals (CRSs) or UE-RSs [240].

This section considers a coordinated cluster of $|\mathcal{B}|=3$ cooperating $120^{\circ}$ sectors and $N_{t}=4$ transmit antennas per sector. In this case, there are $|\mathcal{B}| N_{t}=12$ transmit antennas in the cluster, and it is therefore required to extend the LTE-A CSI-RSs pattern. Figure 7.5 shows the pilot symbol allocation grid proposed for the coordinated cluster under consideration [220]. This figure depicts the positions of the CSI-RSs of the different transmit antennas within the set of used subcarriers. 

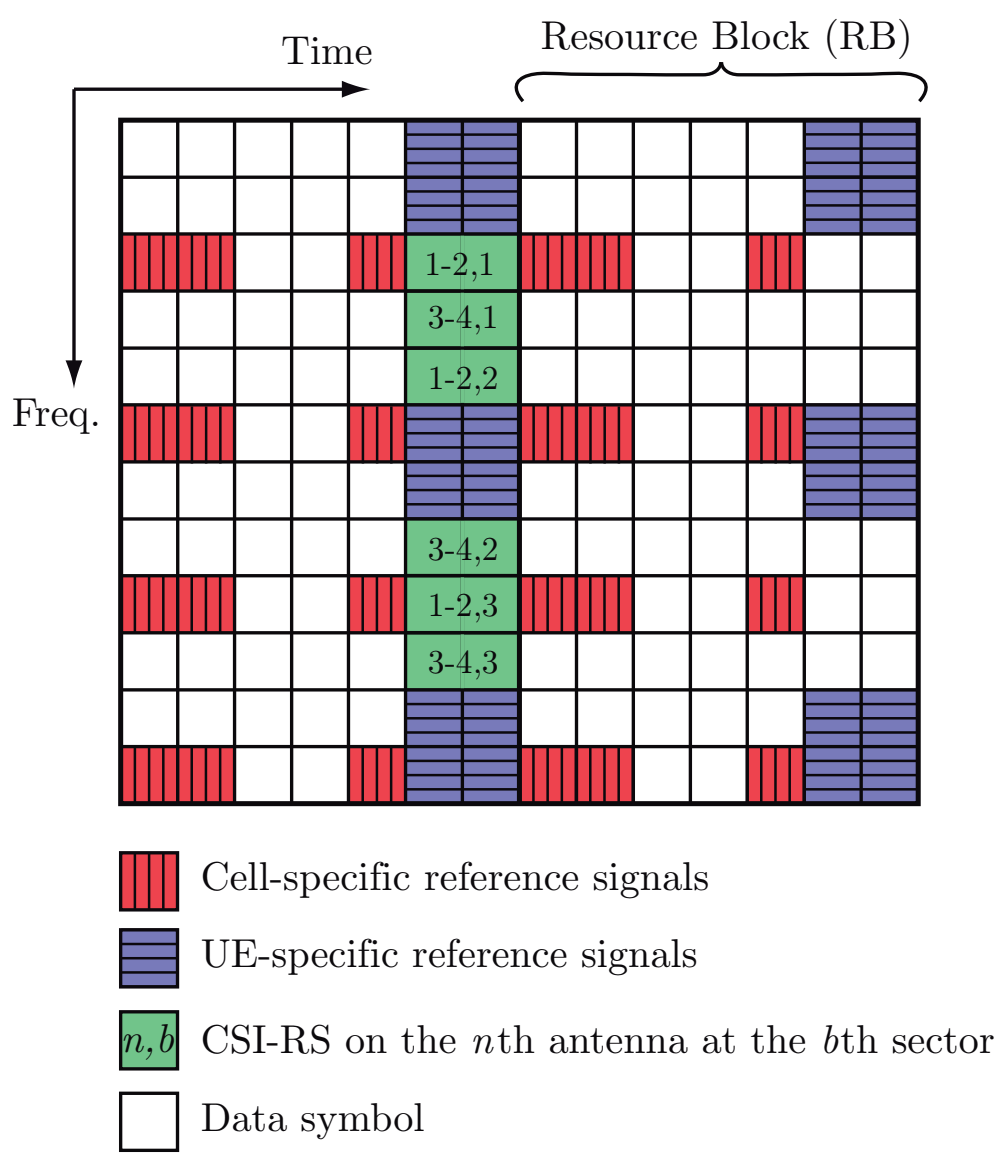

Figure 7.5: Proposed pilot symbol allocation in a resource block (RB) of LTE-Advanced for a cluster of $|\mathcal{B}|=3$ sectors with $N_{t}=4$ transmit antennas per sector [240].

A frequency-division multiplexing (FDM) scheme is used to transmit the CSI-RSs of the different pairs of transmitting antennas (1-2 and 3-4 in each sector) in the coordinated cluster. To separate the RSs of each antenna in the pair, either codedivision multiplexing (CDM) or time-division multiplexing (TDM) could be used. In particular, TDM with code length spanning on two resource elements in time domain is proposed in Rel. 10 [240].

More advanced pilot allocation schemes using a combination of FDM, TDM and CDM are presented in [278-280]. However, the evaluation of the different pilot allocation schemes is out of the scope of this thesis. Therefore, it is assumed that the MSs obtains an error-free channel estimation through a simple LS channel estimation [11, Chap. 8]. It should be pointed out that the presence of a guard 
Table 7.2: Channel and system parameters

\begin{tabular}{|l|l|}
\hline Parameter & Value \\
\hline Inter-site distance & $500 \mathrm{~m}$ \\
Channel model & 3GPP SCME \\
Channel scenario & Suburban Macro \\
Number of paths $(S)$ & 6 \\
Carrier frequency & $2 \mathrm{GHz}$ \\
Sampling frequency $\left(f_{s}\right)$ & $30.72 \mathrm{MHz}$ \\
Bandwidth & $20 \mathrm{MHz}$ \\
CP length $(\mu$ s / samples) & $4.69 / 144$ \\
Shadowing standard deviation & $8 \mathrm{~dB}$ \\
Number of subcarriers $(K)$ & 2048 \\
Number of modulated subcarriers $\left(K_{\mathrm{m}}\right)$ & 1200 \\
Number of used RB & 100 \\
Number of coordinated sectors in the cluster $(|\mathcal{B}|)$ & 3 \\
BS antennas per sector $\left(N_{t}\right)$ & 4 \\
BS antenna spacing & $\lambda / 2$ \\
MSs number $(M)$ & 8 \\
Signal constellation & $64-\mathrm{QAM}$ \\
\hline
\end{tabular}

band with unmodulated subcarriers causes an ill conditioning problem in the LS estimation. Thus, different solutions, such as the ones presented in [281, 282], need to be applied in order to achieve an accurate estimation.

\subsubsection{Numerical results}

This section presents simulation results comparing the performance of the limited feedback scheme based on DQ to another scheme where the real and imaginary parts of the channel coefficients are quantized considering a Gaussian PDF. This scheme is referred to as Gaussian quantization (GQ) scheme. In addition, the effect of the quantized CSIT over the different precoding algorithms presented in Chapter 4 has been evaluated.

A macrocell deployment model is considered with parameters as specified in [182, 276] and collected in Table 7.2. Therefore, the statistical analysis carried out in Section 7.1.2 is valid for this channel. The channel follows a block fading model, remaining constant between one channel estimation period and its following one. The sum-power constraint has been equally allocated over all subcarriers and MSs are randomly distributed over the coordinated cluster area (see Fig. 7.4). 


\section{Performance of limited feedback schemes}

This section discusses the relation between the number of bits employed to quantize the different parameters in the DQ scheme and its performance.

The performance of the proposed quantizer for different bit allocations has been evaluated through Monte Carlo simulations. The GQ scheme has also been evaluated for comparison. In this scheme, $B_{G}$ bits are used to quantize the real and imaginary parts of each coefficient of the CIR. A similar scheme, but using uniform quantization instead of Gaussian quantization is proposed in [161]. It is important to observe that the optimizations applied to the scheme in [161], such as different bit allocation across the different paths, could also be applied to the DQ and GQ schemes. However, this comparison has been performed with equal bit allocation across the different paths.

The metric used to compare both feedback schemes is the cost, which indicates the total number of bits that each MS uses to quantize the CIR of all the transmit antennas in the cluster. The cost for both schemes can be expressed as:

$$
\begin{gathered}
C_{\mathrm{DQ}}=\left\{2 B_{R} \sum_{b=1}^{|\mathcal{B}|} L_{b}+\left(B_{M}+B_{P}\right)\left(N_{t}-1\right) \sum_{b=1}^{|\mathcal{B}|} L_{b}\right\}+\left\{N_{D} \sum_{b=1}^{|\mathcal{B}|}\left(L_{b}-1\right)\right\}, \\
C_{\mathrm{GQ}}=\left\{2 B_{G} N_{t} \sum_{b=1}^{|\mathcal{B}|} L_{b}\right\}+\left\{N_{D} \sum_{b=1}^{|\mathcal{B}|}\left(L_{b}-1\right)\right\},
\end{gathered}
$$

where $L_{b}$ is the number of resolvable paths of the channel between each user and the $b$ th sector array and $N_{D}$ is the number of bits dedicated to quantize the discrete delay of the different paths. The first term in (7.8) and (7.9) collects the cost related to quantize the channel coefficients of the paths, whereas the second term collects the cost of quantizing the discrete delays, which is the same for both schemes.

Since there is not any standardization regarding the number of bits that should be used to quantize explicit feedback, the explicit feedback scheme in IEEE 802.11n has been adopted as a reference [13]. In this scheme, $4-8$ bits are used to quantize the real and imaginary parts of the entries of the CSI matrices. In the case of the GQ scheme, $N_{D}=5$ bits and $B_{G}=5-8$ bits, involving a cost ranging from 675 to 1025 bits per MS approximately. This range of costs would be affordable in a system as the CoMP field testbed presented in [71].

On the other hand, many different configurations of the DQ scheme varying the number of bits given to $B_{R}, B_{M}$ and $B_{P}$ have been evaluated in [220]. Table 7.3 collects those configurations that show the best performance in the same range of cost as the GQ scheme. Once $B_{R}$ has been determined, these configurations 
Table 7.3: Configurations of the Differential Quantizer.

\begin{tabular}{|c|c|c|c|c|c|}
\hline$\sharp$ & $B_{R}$ & $B_{M}$ & $B_{P}$ & Cost & MSE \\
\hline \hline 1 & 7 & 3 & 5 & 643 & 0.0563 \\
\hline 2 & 7 & 3 & 6 & 687 & 0.0348 \\
\hline 3 & 7 & 4 & 6 & 732 & 0.0180 \\
\hline 4 & 7 & 4 & 7 & 777 & 0.0124 \\
\hline 5 & 7 & 5 & 7 & 821 & 0.0071 \\
\hline 6 & 8 & 5 & 7 & 850 & 0.0060 \\
\hline 7 & 7 & 6 & 7 & 865 & 0.0058 \\
\hline 8 & 8 & 6 & 7 & 895 & 0.0043 \\
\hline 9 & 8 & 6 & 8 & 939 & 0.0031 \\
\hline 10 & 8 & 7 & 8 & 985 & 0.0023 \\
\hline
\end{tabular}

can be approximately obtained considering the magnitude ratio and the phase as independent variables and using the GBA algorithm presented in Algorithm 5. The reference antenna in each sector array is chosen between $n^{\prime}=2$ and $n^{\prime}=3$ depending on the magnitude of $\hat{h}_{n^{\prime} s}$. The MSE is defined as

$$
\mathrm{MSE}=\mathbb{E}\left[\left\|\hat{\mathbf{h}}_{m}[k]-\mathbf{h}_{m}[k]\right\|^{2}\right]=|\mathcal{B}| N_{t} \sigma_{e}^{2}
$$

where $\mathbf{h}_{m}[k]$ represents the CFR vector for the $m$ th MS on the $k$ th subcarrier, $\hat{\mathbf{h}}_{m}[k]$ represents its quantized version and $\sigma_{e}^{2}$ was introduced in (4.1). The values of the MSE column have been obtained from the simulation.

In order to compare the performance of GQ and DQ, Fig. 7.6 shows the MSE obtained with both feedback schemes for the configurations stated before. It can be observed that the DQ scheme offers more flexibility regarding the bit allocation, which allows for a larger number of cost-MSE combinations than GQ. The dashed line represents the LS regression of the MSE achieved by the different DQ configurations in terms of cost. In this regard, the MSE achieved by the DQ scheme results in approximately half the cost of the GQ scheme. Setting a particular MSE target, the figure shows that around 75 bits/MS can be saved every feedback period by using DQ instead of GQ. This reduction can represent up to $10 \%$ of the total amount of the feedback channel information.

In summary, DQ outperforms GQ in terms of MSE with respect to the same number of bits and shows higher flexibility regarding feedback bit allocation. 


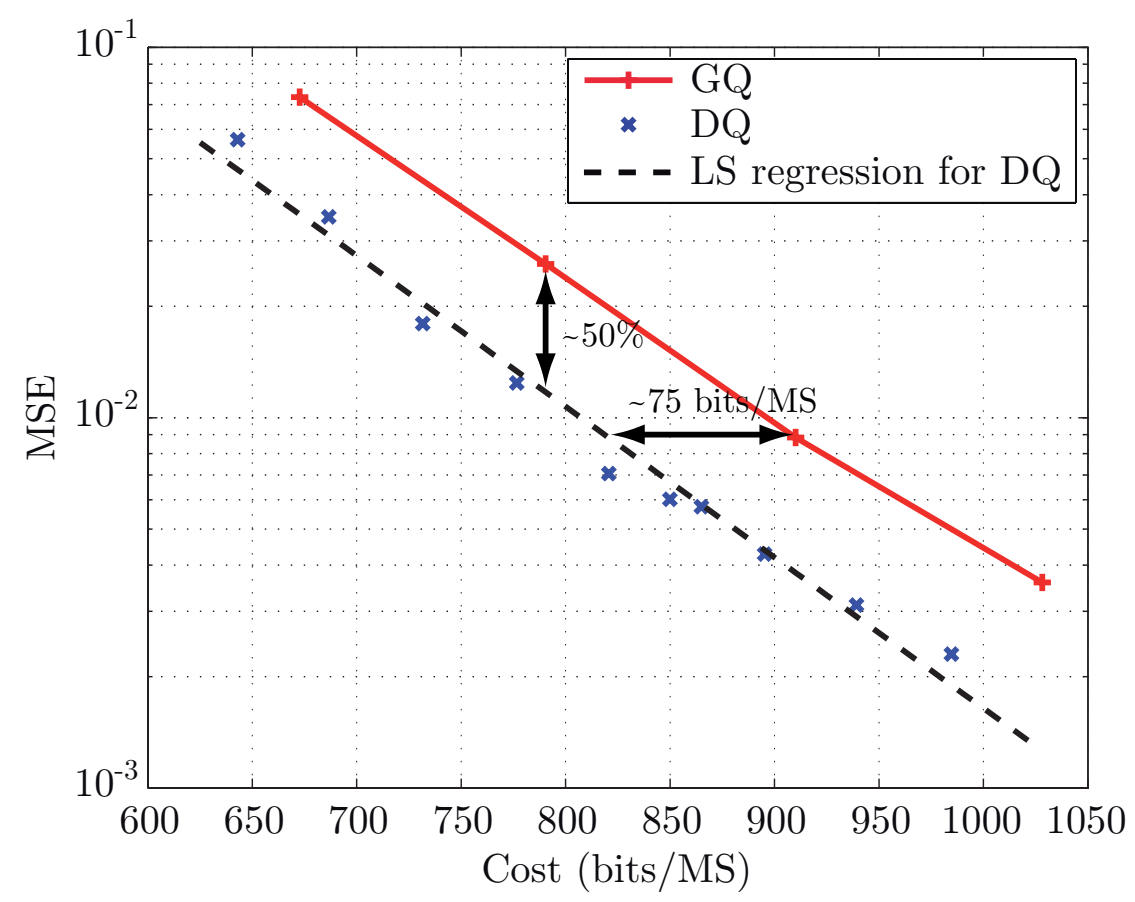

Figure 7.6: MSE vs. cost for GQ and DQ feedback schemes.

\section{Performance of precoding techniques}

In the previous subsection, different feedback bit allocations for the DQ scheme were analyzed, carrying out a performance comparison between that scheme and the GQ schemes in terms of MSE. However, from a practical point of view, it is more interesting to evaluate the cluster performance in terms of BER and sumrate. This section compares how the different precoding techniques perform with the limited feedback schemes under evaluation. Note that either BER or sumrate could be further improved by means of power allocation. Nevertheless, these techniques may result in some users being dropped due to their channel condition, therefore no particular power allocation technique is used. Simulation results are shown in terms of SNR. The transmit sum-power $P$ for the different SNR values has been calculated assuming an MS placed at the cell-edge, taking into account the propagation characteristics of the channel and the thermal noise.

As seen in Chapter 4, BER and sum-rate depend on the variance of the additive error of the channel, $\sigma_{e}^{2}$, among other parameters. Using (7.10), the configurations of Table 7.3 for the DQ scheme and Fig. 7.6, it can be stated that $\sigma_{e}^{2}$ in both GQ and DQ feedback schemes can be approximated through LS fitting by the following 


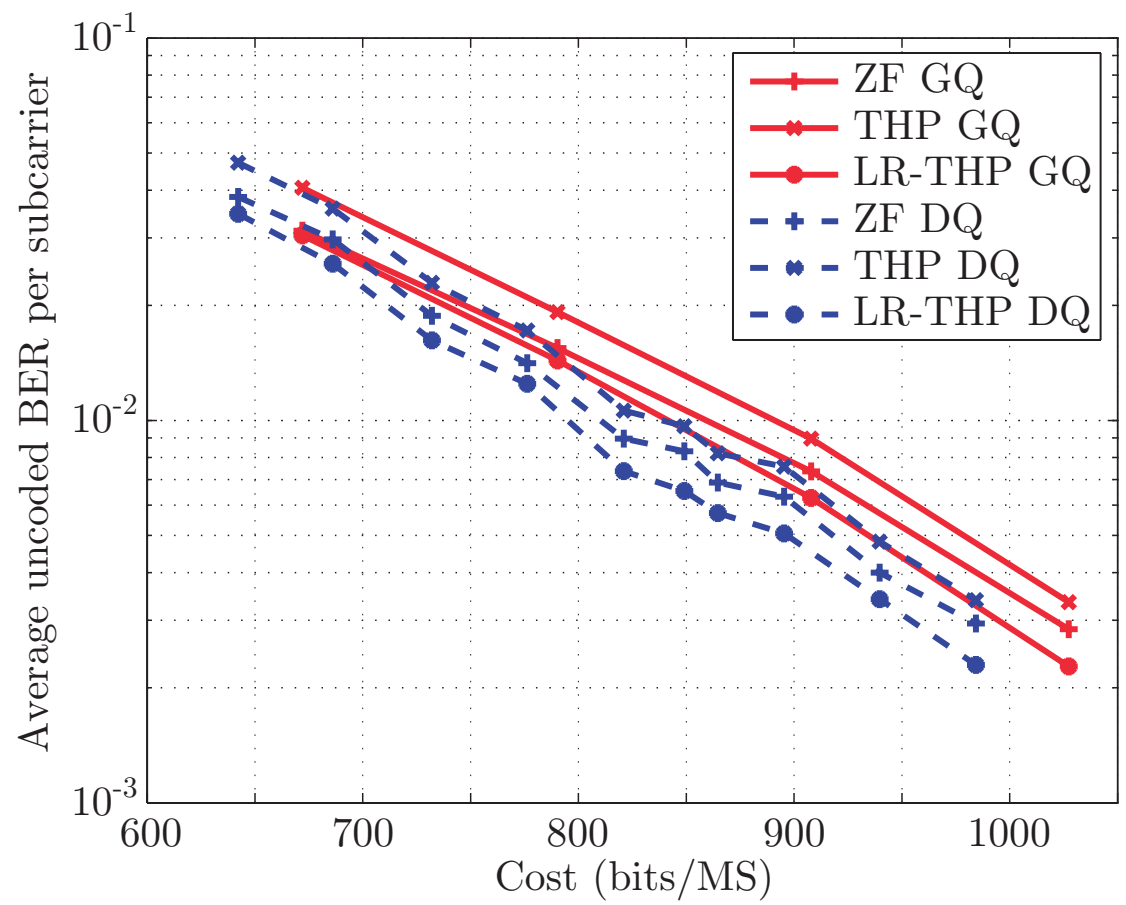

Figure 7.7: Average uncoded BER per subcarrier for ZF, THP and LR-THP with GQ and DQ feedback schemes and $\mathrm{SNR}=30 \mathrm{~dB}$.

expression:

$$
\sigma_{e}^{2}=a \cdot 10^{b C},
$$

where $C$ represents the cost expressed as the number of bits per MS and $a$ and $b$ are fitting parameters. For the GQ scheme, $a \approx 1.860$ and $b \approx-3.712 \cdot 10^{-3}$, whereas for the DQ scheme, $a \approx 1.612$ and $b \approx-4.073 \cdot 10^{-3}$. From this result, it can be seen that the GQ scheme has a slightly larger slope, although the difference between them remains almost constant.

Figure 7.7 compares the BER performance of ZF, THP and LR-THP techniques in a system with these limited feedback schemes, channels generated according Table 7.2 and a system SNR of $30 \mathrm{~dB}$. The results show an almost linear relation between $\log _{10}(\mathrm{BER})$ and the cost for GQ. However, the traces for DQ present some fluctuations. This is due to the fact that MSE and $\sigma_{e}^{2}$ do not strictly follow a line for the selected configurations in Fig. 7.6. Nevertheless, it is important to point out that these configurations outperform the GQ scheme for any given cost, regardless of the precoding technique. Using the DQ scheme instead of using the GQ scheme allows savings of up to 75 bits/MS for configurations with a BER between $10^{-2}$ and $10^{-3}$. 


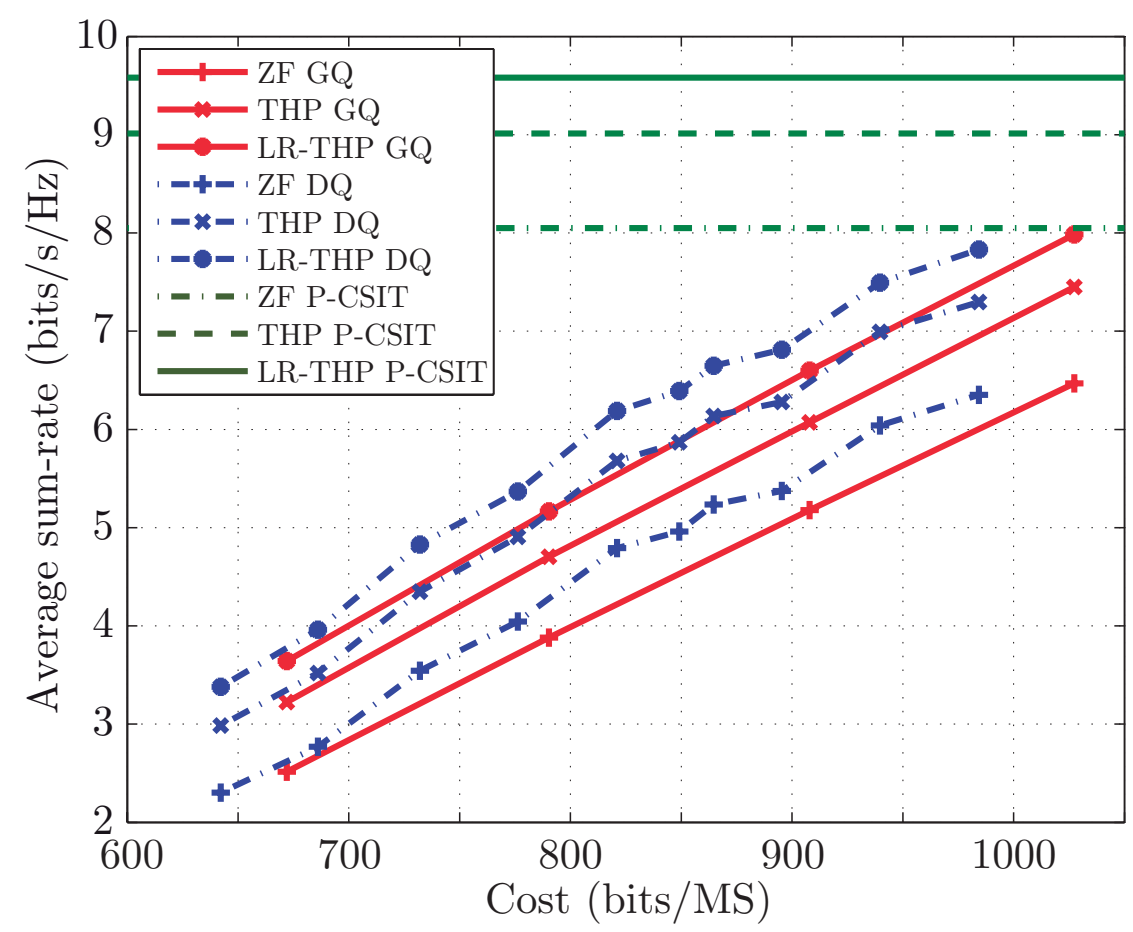

Figure 7.8: Average sum-rate per subcarrier for ZF, THP and LR-THP with GQ, DQ and Perfect CSIT and SNR $=30 \mathrm{~dB}$.

Figure 7.8 shows the results in terms of the average cluster sum-rate. As in Fig. 7.7, it can be seen that the sum-rate obtained for the DQ scheme is not completely linear with the cost. For a given sum-rate and precoding technique, around 50 bits/MS can be saved by using the DQ scheme instead of the GQ scheme. This figure also shows a trade-off between processing complexity and feedback information to increase the sum-rate.

Figures 7.9 and 7.10 show the BER and sum-rate achieved by the cluster under consideration for the GQ and DQ schemes and different system SNRs, respectively. The first scheme uses GQ with $B_{G}=7$ bits whereas the second scheme uses DQ with $B_{R}=7, B_{M}=6$ and $B_{P}=8$ bits. These two configurations have been chosen due to their similar cost (around 910 bits/MS).

Figure 7.9 shows that the DQ scheme achieves lower BER for all SNR values than the GQ scheme, providing a gain between 2 and $4 \mathrm{~dB}$ for system SNRs ranging from 10 to $20 \mathrm{~dB}$. For SNRs higher than $20 \mathrm{~dB}$, the BER remains constant although the system SNR increases. Considering the SINR expressions (4.6), (4.14) and (4.19), it is interesting to calculate the $\mathrm{SNR}$ value so that $\sigma_{e}^{2}=1 / P$. This 


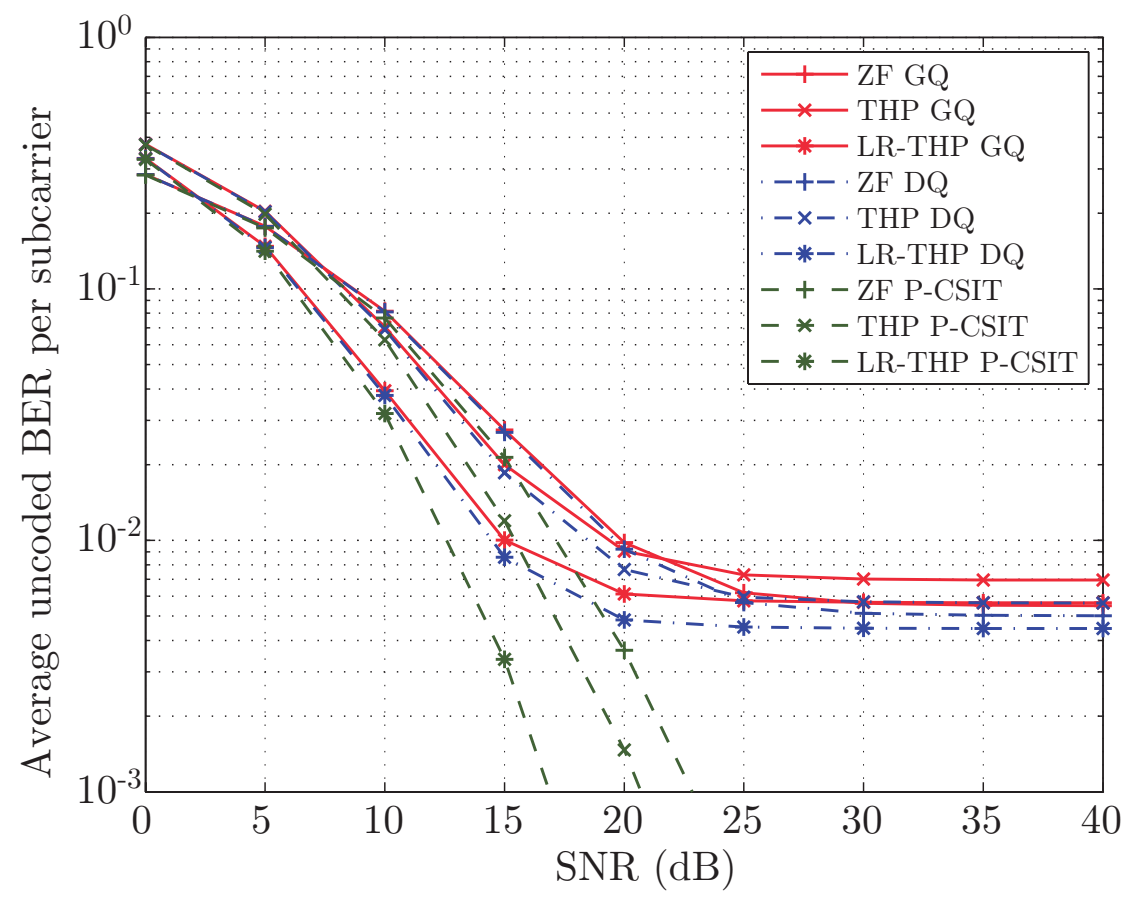

Figure 7.9: Average uncoded BER per subcarrier for ZF, THP and LR-THP with GQ $\left(B_{G}=7\right)$, DQ $\left(B_{R}=7, B_{M}=6, B_{P}=8\right)$ and Perfect CSIT.

happens for $\mathrm{SNR} \approx 14.3$ and $\mathrm{SNR} \approx 17.3$ when using the GQ and $\mathrm{DQ}$ scheme, respectively. Next, it can be considered that $\sigma_{e}^{2} \gg 1 / P$ for $\mathrm{SNR}>20$, so the system is interference-limited in this region due to the imperfect CSIT. The same behavior can be observed in Fig. 7.10, where the growth of the sum-rate starts to decrease for SNRs higher than $20 \mathrm{~dB}$. Here, DQ schemes can achieve a gain up to $5 \mathrm{~dB}$ over GQ.

With regard to the different precoding techniques under the imperfect CSIT provided by the GQ and DQ feedback schemes, simulation results validate the analysis performed in Chapter 4. Figure 7.9 shows that LR-THP can provide a gain of around $4 \mathrm{~dB}$ over THP for a given BER, whereas THP outperforms ZF with a gain of around $2 \mathrm{~dB}$. It is also interesting to point out that the different precoding techniques also achieve different levels of BER floor for SNR higher than $20 \mathrm{~dB}$. Although Fig. 7.10 shows similar results in terms of sum-rate for all the precoding techniques, it can be observed that THP performs closer to LR-THP than to ZF. In the noise limited region (from 15 to $25 \mathrm{~dB}$ ), LR-THP provides a gain of around $3 \mathrm{~dB}$ over THP, whereas the gain of THP over ZF increases to more than $5 \mathrm{~dB}$. 


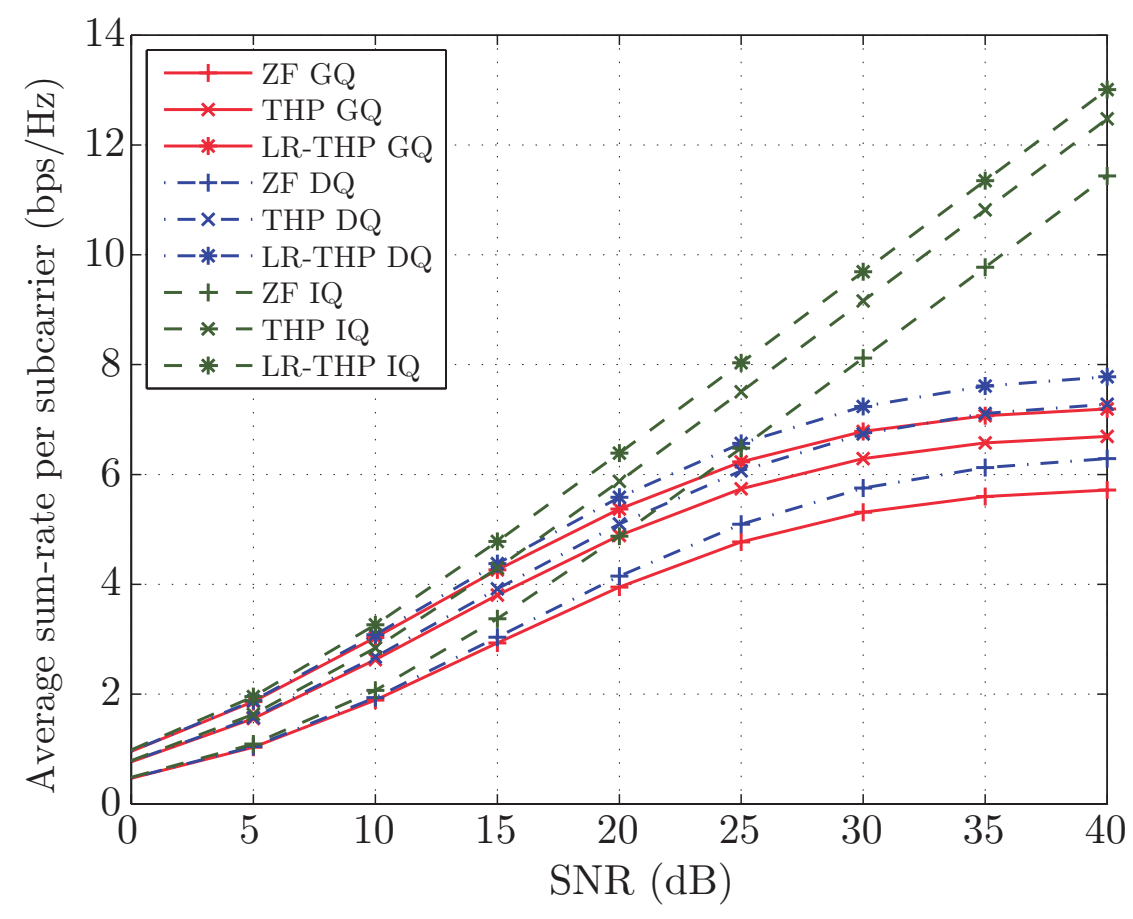

Figure 7.10: Average sum-rate per subcarrier for ZF, THP and LR-THP with GQ $\left(B_{G}=7\right)$, DQ $\left(B_{R}=7, B_{M}=6, B_{P}=8\right)$ and Perfect CSIT.

\subsubsection{Conclusion}

This section has presented a low-complexity limited feedback scheme for highly correlated environments. The scheme is based on the spatial statistical characterization of the SCM and has been evaluated in a coordinated cluster. The channel estimation is performed using the proposed pilot symbol allocation grid for a coordinated cluster and the CSI is fed back through the proposed scheme. This scheme takes advantage of the spatial correlation between antennas without requiring a statistical knowledge of the channel or a higher computational complexity. Its performance has been compared to the GQ scheme, which is based on Gaussian quantization without considering spatial correlation. The simulation results show that the proposed scheme outperforms the GQ scheme in terms of MSE, offering a higher flexibility regarding feedback bit allocation.

The effect of imperfect CSIT due to the limited feedback scheme has been evaluated on different precoding schemes: ZF, THP and LR-THP. The proposed scheme achieves a higher sum-rate than the GQ scheme for the same number of feedback bits. Simulation results also show that the proposed scheme achieves a better 
performance in terms of sum-rate and BER when ZF, THP or LR-THP techniques are used. Among the evaluated precoding techniques, numerical results show that the highest robustness against imperfect CSI is achieved with LR-THP at the cost of a higher complexity.

\subsection{Quantization based on the spatial characterization of the Kronecker correlation model}

The previous section has shown that the statistical analysis of the spatial correlation in the SCM is a very difficult task due to the complexity of the model and the large number of parameters involved in the channel generation. For this reason, the statistical characterization was only possible for a high correlation. In order to obtain a more general statistical characterization, a simpler channel model is necessary.

In this section, the statistical characterization of the spatial downlink channel in a MU-MISO system assuming the Kronecker correlation model is presented. In this characterization, one of the antennas in the array is also chosen as the reference antenna and the channel fading from this antenna is considered to be the reference channel fading. The rest of the channel fading coefficients, known as non-reference channel fading, are statistically characterized by their envelope and phase taking into account the reference antenna fading and the corresponding correlation coefficient.

Two different channel quantization schemes that make use of this statistical characterization to reduce the feedback information are presented. In the proposed schemes, the envelope and phase of the reference channel fading are quantized considering a Rayleigh distribution and a uniform distribution, respectively [174, 283]. For the non-reference channel fading coefficients, the quantization is performed considering their PDF, which in turn depend on the reference channel fading and the spatial correlation between each channel fading and the reference one. The difference between the proposed schemes is related to the reference channel fading. Whereas in one of the proposed schemes the reference channel fading is fixed, in the other scheme the reference channel fading changes along the quantization process.

The proposed schemes have been compared to a scheme based on standard polar quantization (PQ), where the spatial correlation is not taken into account, and a scheme based on the KL transform, that achieves maximum decorrelation of Gaussian sources. The main advantage of the proposed schemes is that, instead of having to send back the entire correlation matrix, only the correlation coefficients between the reference channel fading and each of the non-reference fading coefficients need to be sent back. This approach can offer significant sav- 
ings in feedback overhead. Numerical results have been obtained considering the SCM [182]. Results show that the proposed schemes outperform the KL scheme and the scheme based on standard polar quantization in highly and moderately correlated scenarios. Thus, the contributions in this section can be summarized as follows:

- Statistical envelope and phase characterizations of the non-reference channel fading coefficients given the reference fading are presented using the results of $[114,284]$. These characterizations, which focus on the channel quantization design, include the expressions of the raw moments needed for the codebook generation. An approximation of the phase difference distribution presented in [284] showing a lower computational complexity is also proposed.

- Two different channel quantization schemes that make use of the envelope and phase statistical characterizations are presented. These schemes exploit the spatial correlation in order to reduce the feedback information. Comparisons to similar quantization schemes are shown and a discussion about the performance in terms of MSE, complexity and required feedback is also provided.

\subsubsection{System model}

A narrowband MU-MISO FDD communication system is considered, with a single BS that simultaneously transmits to multiple MSs using spatial multiplexing. The BS is equipped with a uniform linear array of $N_{t}$ antennas with an antenna separation $d$, and the MSs have a single antenna. Each MS is assumed to obtain an error-free channel estimation and has to send back the quantized version of the estimated channel through the feedback channel, since this information will be necessary for scheduling and precoding tasks at the BS. Focusing on a given MS, the received signal can be expressed as

$$
y=\mathbf{h}^{H} \mathbf{x}+n,
$$

where vector $\mathbf{h}=\left[h_{1}, \ldots, h_{N_{t}}\right]^{H} \in \mathbb{C}^{N_{t} \times 1}$ is composed of the channel fading coefficients between each antenna in the BS and the antenna in the MS, vector $\mathbf{x} \in \mathbb{C}^{N_{t} \times 1}$ represents the signal transmitted by the BS and is subject to a power constraint $\mathbb{E}\left[\|\mathbf{x}\|^{2}\right] \leq P_{\mathrm{T}}$, and $n$ is the noise component, which follows a circularly-symmetric complex Gaussian distribution with zero mean and unit variance, $\mathcal{C N}(0,1)$. Using the Kronecker correlation model, the channel vector can be expressed as $[174,285]$

$$
\mathbf{h}=\mathbf{C}_{\mathrm{s}}^{1 / 2} \mathbf{g}
$$

where $\mathbf{C}_{\mathrm{s}}=\mathbb{E}\left[\mathbf{h h}^{H}\right] \in \mathbb{C}^{N_{t} \times N_{t}}$ is the spatial correlation matrix at the transmitter and $\mathbf{g} \in \mathbb{C}^{N_{t} \times 1}$ is a vector whose elements are i.i.d. circularly-symmetric complex 
Gaussian variables, with zero mean and unit variance, $\mathcal{C N}(0,1)$. Previous research has shown that the Kronecker model results in poor estimates for capacity [178]. However, this model is not used to evaluate the channel capacity, but to study the effect of the spatial correlation at the transmitter in the feedback scheme.

\subsubsection{Statistical characterization of the spatial channel}

This section presents the statistical characterization of the channel vector modeled in (7.13). First, a system with only $N_{t}=2$ transmit antennas is considered. However, once the statistical characterization is stated, it is extended to the case of $N_{t}>2$ antennas. It is important to note that, due to the separable correlation assumed in the Kronecker model [174], this analysis can be straightforwardly extended when the MSs have multiple antennas and the correlation is also present at the receiver. Let $\mathbf{g}=\left[g_{1}, g_{2}\right]^{T}$ be a vector with i.i.d. elements, as stated in (7.13), and let $\mathbf{C}_{\mathrm{s}}$ be the spatial correlation matrix at the transmitter, given by

$$
\mathbf{C}_{\mathrm{s}}=\left[\begin{array}{cc}
1 & \rho^{*} \\
\rho & 1
\end{array}\right]
$$

Following (7.13) and (7.14), the elements of vector $\mathbf{h}$ can be expressed as

$$
\begin{aligned}
& h_{1}=k_{1} g_{1}+k_{2}^{*} g_{2} \\
& h_{2}=k_{2} g_{1}+k_{1} g_{2},
\end{aligned}
$$

where $k_{1}$ and $k_{2}$ are given by

$$
\begin{aligned}
& k_{1}=\frac{\sqrt{1+|\rho|}+\sqrt{1-|\rho|}}{2} \in \mathbb{R}^{+} \\
& k_{2}=\frac{\rho(\sqrt{1+|\rho|}-\sqrt{1-|\rho|})}{2|\rho|} \in \mathbb{C} .
\end{aligned}
$$

It can be noted that, for arbitrary correlated channels, $k_{1}^{2}+\left|k_{2}\right|^{2}=1$. For uncorrelated channels $(|\rho|=0)$, the previous equations become $k_{1}=1$ and $\lim _{|\rho| \rightarrow 0} k_{2}=0$, while for highly correlated channels $(|\rho| \approx 1), k_{1} \approx\left|k_{2}\right| \approx 1 / \sqrt{(2)}$. Thus, since $h_{1}$ and $h_{2}$ are a linear combination of i.i.d. $\mathcal{C N}(0,1)$ random variables, they will also show a $\mathcal{C N}(0,1)$ distribution. The real and imaginary parts of $h_{1}$ and $h_{2}$ have equal power given by

$$
\mathbb{E}\left[\left(\mathfrak{R e}\left\{h_{1}\right\}\right)^{2}\right]=\mathbb{E}\left[\left(\mathfrak{I m}\left\{h_{1}\right\}\right)^{2}\right]=\mathbb{E}\left[\left(\mathfrak{R e}\left\{h_{2}\right\}\right)^{2}\right]=\mathbb{E}\left[\left(\mathfrak{I m}\left\{h_{2}\right\}\right)^{2}\right]=\frac{1}{2}
$$


The covariances between the real and imaginary parts of $h_{1}$ and $h_{2}$ are given by the following relationships:

$$
\begin{aligned}
& \mathbb{E}\left[\mathfrak{R e}\left\{h_{1}\right\} \mathfrak{I m}\left\{h_{1}\right\}\right]=\mathbb{E}\left[\mathfrak{R e}\left\{h_{2}\right\} \mathfrak{I m}\left\{h_{2}\right\}\right]=0 \\
& \mathbb{E}\left[\mathfrak{R e}\left\{h_{1}\right\} \mathfrak{R e}\left\{h_{2}\right\}\right]=\mathbb{E}\left[\mathfrak{I m}\left\{h_{1}\right\} \mathfrak{I m}\left\{h_{2}\right\}\right]=k_{1} \mathfrak{R e}\left\{k_{2}\right\}=\frac{\mathfrak{R e}\{\rho\}}{2} \\
& \mathbb{E}\left[\mathfrak{R e}\left\{h_{1}\right\} \mathfrak{I m}\left\{h_{2}\right\}\right]=-\mathbb{E}\left[\mathfrak{I m}\left\{h_{1}\right\} \mathfrak{R e}\left\{h_{2}\right\}\right]=k_{1} \mathfrak{I m}\left\{k_{2}\right\}=\frac{\mathfrak{I m}\{\rho\}}{2} .
\end{aligned}
$$

\section{Envelope statistical characterization. Case $N_{t}=2$}

Let us define, without loss of generality, transmit antenna number 2 as the reference antenna and antenna number 1 as the non-reference antenna, and their corresponding reference and non-reference channel fading coefficients as $h_{\mathrm{r}}=h_{2}$ and $h_{\mathrm{nr}}=h_{1}$. The envelope of each channel shows the following Rayleigh distribution,

$$
\begin{aligned}
f_{r_{\mathrm{r}}}\left(r_{\mathrm{r}}\right) & =\frac{r_{\mathrm{r}}}{b_{\mathrm{R}}^{2}} \exp \left(-\frac{r_{\mathrm{r}}^{2}}{2 b_{\mathrm{R}}^{2}}\right) \\
f_{r_{\mathrm{nr}}}\left(r_{\mathrm{nr}}\right) & =\frac{r_{\mathrm{nr}}}{b_{\mathrm{R}}^{2}} \exp \left(-\frac{r_{\mathrm{nr}}^{2}}{2 b_{\mathrm{R}}^{2}}\right),
\end{aligned}
$$

where $r_{\mathrm{r}}=\left|h_{\mathrm{r}}\right|, r_{\mathrm{nr}}=\left|h_{\mathrm{nr}}\right|$ and $b_{\mathrm{R}}=\sqrt{1 / 2}$ is the parameter of the Rayleigh distribution.

As seen in [114], the joint probability distribution of the two envelopes, $r_{\mathrm{r}}$ and $r_{\mathrm{nr}}$, can be expressed by means of the bivariate Rayleigh distribution as

$$
f\left(r_{\mathrm{nr}}, r_{\mathrm{r}}\right)=\frac{r_{\mathrm{nr}} r_{\mathrm{r}}}{b_{\mathrm{R}}^{4}\left(1-|\rho|^{2}\right)} \exp \left(-\frac{r_{\mathrm{nr}}^{2}+r_{\mathrm{r}}^{2}}{2 b_{\mathrm{R}}^{2}\left(1-|\rho|^{2}\right)}\right) I_{0}\left(\frac{r_{\mathrm{nr}} r_{\mathrm{r}}|\rho|}{b_{\mathrm{R}}^{2}\left(1-|\rho|^{2}\right)}\right),
$$

where $I_{0}(\cdot)$ is the modified Bessel function of the first kind of order 0. From (7.23) and (7.25), the conditional probability density function (CPDF) of $r_{\mathrm{nr}}$ given $r_{\mathrm{r}}$ and correlation coefficient $\rho$ can be expressed as $^{1}$

$$
f\left(r_{\mathrm{nr}} \mid r_{\mathrm{r}}, \rho\right)=\frac{r_{\mathrm{nr}}}{b_{\mathrm{R}}^{2}\left(1-|\rho|^{2}\right)} \exp \left(-\frac{r_{\mathrm{nr}}^{2}+r_{\mathrm{r}}^{2}|\rho|^{2}}{2 b_{\mathrm{R}}^{2}\left(1-|\rho|^{2}\right)}\right) I_{0}\left(\frac{r_{\mathrm{nr}} r_{\mathrm{r}}|\rho|}{b_{\mathrm{R}}^{2}\left(1-|\rho|^{2}\right)}\right)
$$

Fig. 7.11 shows the analytical CPDF described by (7.26) and the empirical results, obtained through Monte Carlo simulations with $10^{6}$ channel realizations for different values of reference fading envelopes, $r_{\mathrm{r}}$, and a fixed value of $|\rho|=0.9$. The Rayleigh PDF shown in (7.24) is denoted as Analytical Rayleigh. It can be 


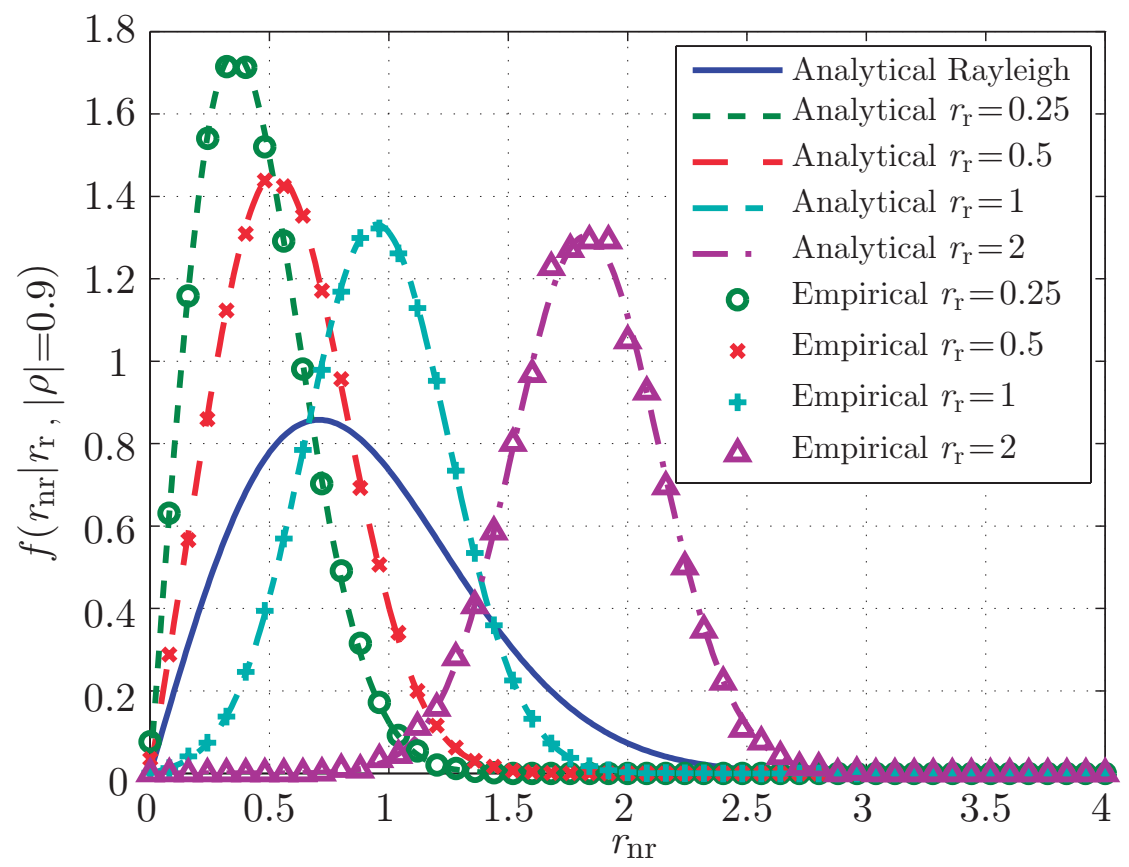

Figure 7.11: CPDF of (7.26) for different envelope values of the reference channel fading, $r_{\mathrm{r}}$, and a fixed value of $|\rho|=0.9$.

observed that the CPDFs of the non-reference fading envelope are located approximately close to the value of the reference due to the high correlation.

Figure 7.12 shows the analytical CPDF of (7.26) and the empirical results, obtained through Monte Carlo simulations with $10^{6}$ channel realizations for a fixed value of $r_{\mathrm{r}}=2$ and different values of the correlation coefficient magnitude, $|\rho|$. In this figure, it can be observed that, as the correlation increases, the PDFs become narrower and closer to the value of the reference fading envelope. It is also important to note that (7.26) is equivalent to (7.24) for uncorrelated antennas $(|\rho|=0)$.

Expressing the modified Bessel function of the first kind of order $0, I_{0}(x)$, through its Taylor series expansion around $x=0$ [286, Sec. 9], the $n$th moment of $f\left(r_{\mathrm{nr}} \mid r_{\mathrm{r}}, \rho\right)$ for a given $r_{\mathrm{r}}$ and $\rho$ can be calculated as

$$
m_{n}=\int_{0}^{\infty} r_{\mathrm{nr}}^{n} f\left(r_{\mathrm{nr}} \mid r_{\mathrm{r}}, \rho\right) \mathrm{d} r_{\mathrm{nr}}=\exp \left(\frac{-C}{A}\right) \sum_{k=0}^{\infty} \frac{C^{k} \Gamma\left(k+\frac{n}{2}+1\right)}{A^{k-\frac{n}{2}}(k !)^{2}}
$$

\footnotetext{
${ }^{1}$ In (7.26), we include the correlation coefficient as a parameter, $f\left(r_{\mathrm{nr}} \mid r_{\mathrm{r}}, \rho\right)$, to explicitly note the dependence of the CPDF on $\rho$.
} 


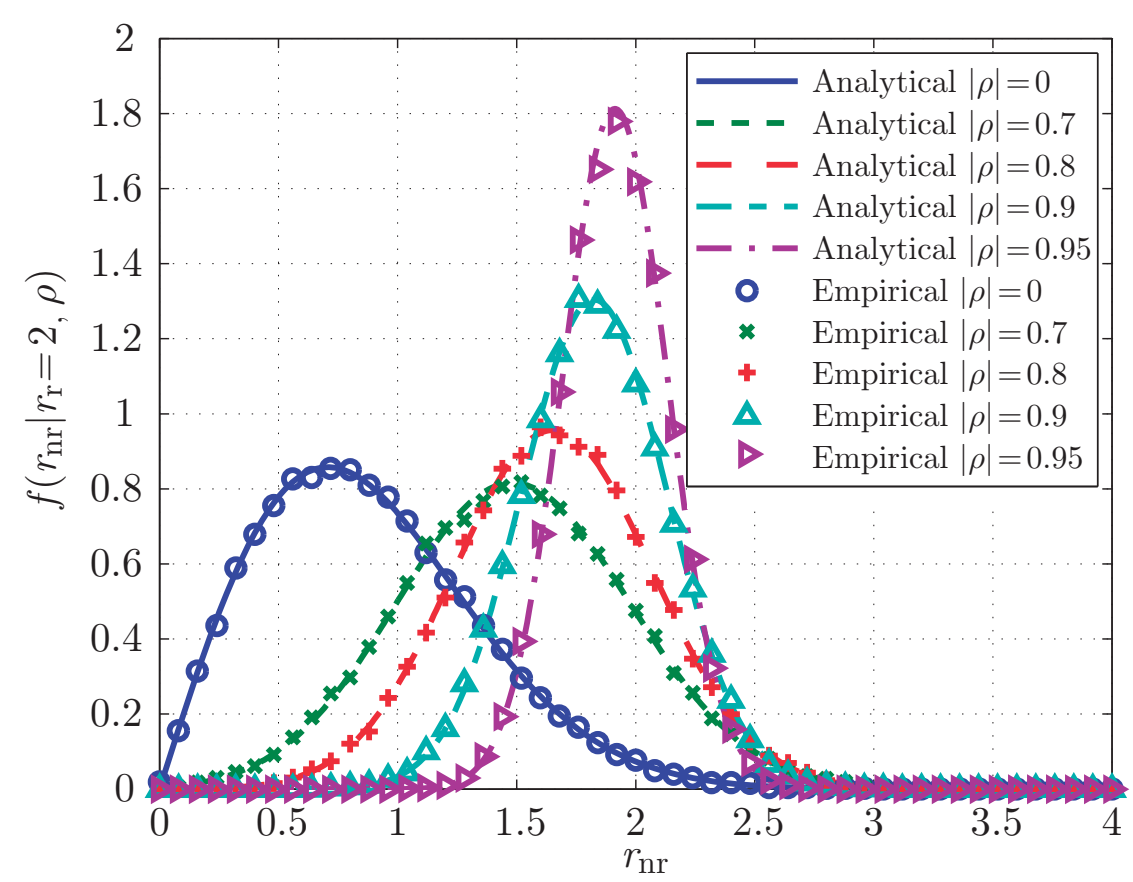

Figure 7.12: CPDF of (7.26) for a fixed value of $r_{\mathrm{r}}=2$ and different values of the correlation coefficient, $|\rho|$.

where $A=2 b_{\mathrm{R}}^{2}\left(1-|\rho|^{2}\right), C=r_{\mathrm{r}}^{2}|\rho|^{2}$ and $\Gamma(\cdot)$ is the Gamma function [286, Sec. 6]. The integral in (7.27) has been calculated in Appendix B within an arbitrary interval $[a, b]$, and its result can be directly applied to interval $[0, \infty]$.

As can be seen in (7.27), the closed expressions of the mean and variance of the PDFs cannot be easily simplified. From Fig. 7.11, it becomes apparent that the variance of the PDF does not significantly change with the reference fading, $r_{\mathrm{r}}$. However, Fig. 7.12 shows that the variance of the non-reference fading decreases and the mean value tends to get closer to the reference fading, $r_{\mathrm{r}}$, as the value of the correlation coefficient increases.

\section{Phase statistical characterization. Case $N_{t}=2$}

The phase of a channel fading follows a uniform distribution in the half-open interval $[-\pi, \pi)$, and its PDF can be expressed as [287]

$$
f_{\theta_{\mathrm{r}}}\left(\theta_{\mathrm{r}}\right)=f_{\theta_{\mathrm{nr}}}\left(\theta_{\mathrm{nr}}\right)= \begin{cases}\frac{1}{2 \pi}, & -\pi \leq \theta<\pi \\ 0, & \text { otherwise }\end{cases}
$$


where $\theta_{\mathrm{r}}=\angle h_{\mathrm{r}}, \theta_{\mathrm{nr}}=\angle h_{\mathrm{nr}}$ and $\theta$ generically denotes either $\theta_{\mathrm{r}}$ or $\theta_{\mathrm{nr}}$.

Equations (7.15) and (7.16) show that the joint probability distribution of the phases, $\theta_{\mathrm{r}}$ and $\theta_{\mathrm{nr}}$, cannot be easily calculated and, to our knowledge, no previous work relating to the statistics of the two phases can be found in the literature. However, considering a high correlation environment with $|\rho| \approx 1, k_{2}$ can be written as

$$
k_{2} \approx e^{j \theta_{\rho}} k_{1}
$$

where $\theta_{\rho}=\angle \rho$. By substituting (7.29) into (7.15) and (7.16), $h_{\mathrm{nr}}$ can be written as a function of $h_{\mathrm{r}}$ expressed as $h_{\mathrm{nr}} \approx e^{-j \theta_{\rho}} h_{\mathrm{r}}$. Thus, the relation between the two phases is given by

$$
\theta_{\mathrm{nr}} \approx \theta_{\mathrm{r}}-\theta_{\rho}
$$

This approximation holds for highly correlated channels, but it includes an error that increases as the correlation decreases. In order to evaluate the error of this approximation, the phase deviation is defined as

$$
\Delta=\theta_{\mathrm{nr}}-\left(\theta_{\mathrm{r}}-\theta_{\rho}\right)
$$

The distribution of the phase deviation $\Delta$ can be obtained from the results in [284]. In our case, the PDF of the phase deviation can be expressed $\mathrm{as}^{2}$

$$
f_{\Delta}(\Delta, \rho)=\frac{2\left(1-|\rho|^{2}\right)}{3 \pi(1-|\rho| \cos \Delta)^{2}}{ }_{2} F_{1}\left(2, \frac{1}{2} ; \frac{5}{2} ;-\frac{1+|\rho| \cos \Delta}{1-|\rho| \cos \Delta}\right),
$$

where ${ }_{2} F_{1}(\cdot, \cdot ; \cdot ; \cdot)$ is the Gaussian hypergeometric function [286, Sec. 15].

Figure 7.13 shows the analytical PDF (7.32) and the empirical results obtained through a Monte Carlo simulation with $10^{4}$ channel realizations for different values of the correlation magnitude, $|\rho|$. It can be noted that, as the correlation coefficient decreases, this PDF tends to exhibit a uniform distribution, as observed in (7.28). On the other hand, its variance decreases as the correlation coefficient increases.

\section{Envelope and phase characterization for $N_{t}>2$}

The previous characterization can be extended to a system with a linear array of $N_{t}>2$ transmit antennas. First, an antenna is selected as a reference. The envelope and the phase of the fading of the reference antenna are characterized by (7.23) and (7.28), respectively. With regard to the $N_{t}-1$ non-reference antennas, the envelope and phase of the $n$th non-reference channel fading are expressed as $r_{\mathrm{nr}}^{(n)}$ and $\theta_{\mathrm{nr}}^{(n)}$, respectively, and they are characterized by their own channel

\footnotetext{
${ }^{2}$ In $(7.32)$, the correlation coefficient is included as a parameter, $f_{\Delta}(\Delta, \rho)$, to explicitly note the dependence of the PDF on $\rho$.
} 


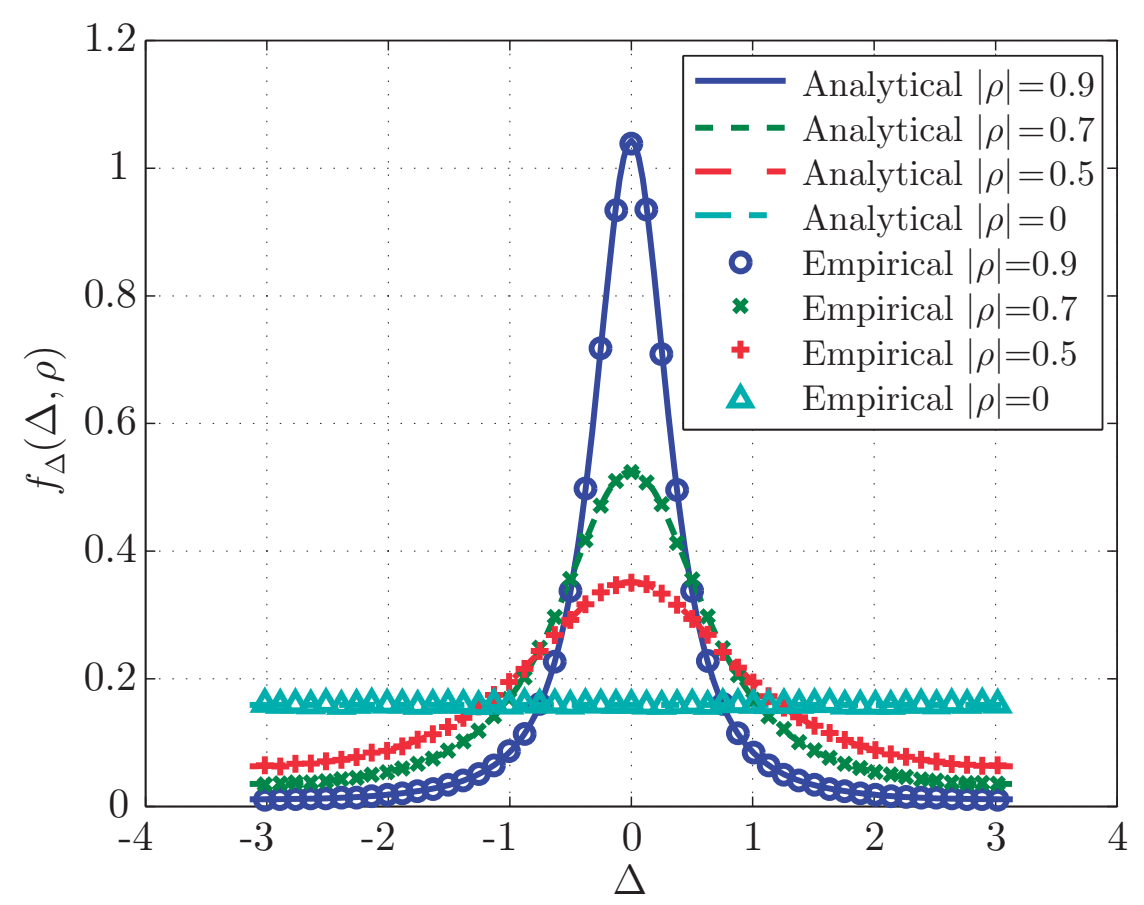

Figure 7.13: PDF of $\Delta$ in (7.32) for different values of the correlation coefficient, $|\rho|$.

fading, the correlation coefficient of the $n$th non-reference antenna with respect to the reference antenna, $\rho_{n}$, and the reference channel fading. Finally, (7.26) defines the statistical characterization of the non-reference fading envelope, $r_{\mathrm{nr}}^{(n)}$, and (7.32) defines the corresponding function of its phase deviation, $\Delta^{(n)}$.

\subsubsection{Proposed quantization scheme}

This section makes use of the statistical characterization discussed in Section 7.2.2 to propose a quantization scheme for a system with an arbitrary number of transmit antennas, $N_{t}$. Uniform polar quantization has been considered in the proposed scheme. The choice of uniform quantization is motivated by a lower complexity with respect to non-uniform quantization in the codebook generation task, while the choice of polar quantization is because the statistical characterization of the channel has been obtained in terms of the envelope and phase of the channel fading coefficients. The steps for the quantization scheme are summarized in Table 7.4. It is important to note that these steps are performed by the different MSs independently of each other. The notation for the number of bits used in the quantization 
of each parameter has also been included in the table, although it will be defined below.

First, a transmit antenna is chosen as a reference. In order to obtain a better quantization performance, the following heuristic formula for the selection of the reference antenna could be used:

$$
N_{\mathrm{r}}=\underset{N_{\mathrm{r}}^{\prime}}{\arg \max } \sum_{\substack{n=1 \\ n \neq N_{\mathrm{r}}^{\prime}}}^{N_{t}}\left|\rho_{n, N_{\mathrm{r}}^{\prime}}\right|, \quad 1 \leq N_{\mathrm{r}}^{\prime} \leq N_{t},
$$

where $N_{\mathrm{r}}$ is the reference antenna and $\rho_{n, N_{\mathrm{r}}^{\prime}}$ is the correlation coefficient between the $n$th and the $N_{\mathrm{r}}^{\prime}$ th antennas. With this strategy, the selected antenna presents the highest cumulative correlation between itself and the rest of the antennas. A large correlation value provides a lower quantization error as can be inferred from Figs. 7.12 and 7.13. The fading experienced from this antenna is denoted as the reference channel fading. The channel fading coefficients from the rest of the antennas are denoted as non-reference channel fading.

The envelope and the phase of the reference channel fading, whose PDFs are given in (7.23) and (7.28) respectively, are quantized using uniform polar quantization ((A.1) and (A.2) in Table 7.4). The non-reference channel fading coefficients are quantized taking advantage of their correlation with the reference channel fading, which is assumed to be known at both receiver and transmitter. Rows (A.3) and (A.4) in Table 7.4 show the quantization of the $n$th non-reference fading. The envelope quantization process considers the CPDF seen in (7.26). It is important to note that the CPDF uses the quantized version $\hat{r}_{\mathrm{r}}$ to quantize $r_{\mathrm{nr}}^{(n)}$, since this parameter is the one that will be available at the BS. In the phase quantization process, the phase deviation $\Delta^{(n)}=\theta_{\mathrm{nr}}^{(n)}-\left(\hat{\theta}_{\mathrm{r}}-\theta_{\rho_{n}}\right)$ is quantized instead of quantizing $\theta_{\mathrm{nr}}^{(n)}$, since $\hat{\theta}_{\mathrm{r}}$ and $\theta_{\rho_{n}}$ are known by the BS. Note that, the PDF depicted in (7.44) is used instead of the PDF depicted in (7.32). This will be discussed below in this section. In what follows, this quantization scheme will be referred to as the conditional quantization (CQ) scheme, since the quantization of the non-reference channel fading coefficients is conditioned by the reference fading.

At the BS, the reference fading can be easily reconstructed from its quantized envelope and phase ((B.1) in Table 7.4). The non-reference fading coefficients, $\hat{h}_{\mathrm{nr}}^{(n)}$, can be calculated using (B.2) in Table 7.4 given the relationship of the phases shown in (7.31). 
Table 7.4: Proposed quantization scheme

A.- MS: channel quantization

Quantized value PDF considered Quantization bits

$\begin{array}{ll}\text { 1.- } & \hat{r}_{\mathrm{r}} \\ \text { 2.- } & \hat{\theta}_{\mathrm{r}} \\ \text { 3.- } & \hat{r}_{\mathrm{nr}}^{(n)} \\ \text { 4.- } & \hat{\Delta}^{(n)}\end{array}$

$B_{M_{\mathrm{r}}}$
$B_{P_{\mathrm{r}}}$
$B_{M_{\mathrm{nr}, n}}$
$B_{P_{\mathrm{nr}, n}}$

B.- BS: channel reconstruction

$$
\begin{array}{ll}
1 .- & \hat{h}_{\mathrm{r}}=\hat{r}_{\mathrm{r}} \exp \left(j \hat{\theta}_{\mathrm{r}}\right) \\
2 .- & \hat{h}_{\mathrm{nr}}^{(n)}=\hat{r}_{\mathrm{nr}}^{(n)} \exp \left(j\left(\hat{\Delta}^{(n)}+\hat{\theta}_{\mathrm{r}}-\theta_{\rho_{n}}\right)\right)
\end{array}
$$

\section{Codebook generation for the reference channel fading}

Uniform polar quantization is used to quantize the reference channel fading. The decision thresholds and the output values for the envelope $\left(r_{\mathrm{r}, m}, \hat{r}_{\mathrm{r}, m}\right)$ and the phase $\left(\theta_{\mathrm{r}, p}, \hat{\theta}_{\mathrm{r}, p}\right)$ of the reference channel fading have been obtained in [288] and are given by:

$$
\begin{array}{rlrl}
r_{\mathrm{r}, m} & =m d_{r} & m & =1, \ldots, M_{\mathrm{r}}-1 \\
\hat{r}_{\mathrm{r}, m} & =(m-1 / 2) d_{r} & m & =1, \ldots, M_{\mathrm{r}} \\
\theta_{\mathrm{r}, p} & =p d_{\theta}-\pi & & p=1, \ldots, P_{\mathrm{r}}-1 \\
\hat{\theta}_{\mathrm{r}, p} & =(p-1 / 2) d_{\theta}-\pi & & p=1, \ldots, P_{\mathrm{r}}
\end{array}
$$

where $r_{0}=0, r_{M_{\mathrm{r}}}=\infty, \theta_{0}=-\pi$ and $\theta_{P_{\mathrm{r}}}=\pi$. Parameters $M_{\mathrm{r}}$ and $P_{\mathrm{r}}$ are the number of quantization levels for envelope and phase, respectively, and the parameters $d_{r}$ and $d_{\theta}$ are the interval sizes. Once $M_{\mathrm{r}}$ and $P_{\mathrm{r}}$ are fixed, the codebook is obtained by minimizing the distortion function with respect to $d_{r}$ and $d_{\theta}$. Since the phase is uniformly distributed, the optimal interval size for the phase is directly obtained with $d_{\theta}=2 \pi / P_{\mathrm{r}}$. Thus, the optimal interval size $d_{r}$ can be obtained through a one-dimensional Newton-Raphson optimization technique over the distortion function [288]

$$
D_{\mathrm{r}}\left(d_{r}\right)=\sum_{m=1}^{M_{\mathrm{r}}} \int_{r_{\mathrm{r}, m-1}}^{r_{\mathrm{r}, m}}\left(r_{\mathrm{r}}^{2}+\hat{r}_{\mathrm{r}, m}^{2}-2 \operatorname{sinc}\left(P_{\mathrm{r}}\right) r_{\mathrm{r}} \hat{r}_{\mathrm{r}, m}\right) f_{r_{\mathrm{r}}}\left(r_{\mathrm{r}}\right) \mathrm{d} r_{\mathrm{r}}
$$

In real systems, parameters such as $M_{\mathrm{r}}$ and $P_{\mathrm{r}}$ are usually considered to be power of 2 integers [13]. Thus, $B_{M_{\mathrm{r}}}=\log _{2} M_{\mathrm{r}}$ and $B_{P_{\mathrm{r}}}=\log _{2} P_{\mathrm{r}}$ are the number of bits dedicated to quantizing the envelope and the phase, respectively, and $B_{\mathrm{r}}=$ $B_{M_{\mathrm{r}}}+B_{P_{\mathrm{r}}}$ is the number of bits dedicated to quantizing the reference channel fading. 


\section{Codebook generation for non-reference channel fading}

The uniform polar quantizer shown in [288] has been used for the reference channel fading. However, Fig. 7.14a shows that a more suitable uniform quantizer for the envelope of non-reference channel fading can be obtained by including a shift parameter, $d_{r 0}$. This parameter shifts the decision thresholds and the output values of the envelope quantizer $d_{r 0}$ units, allowing a smaller or larger first partition. The codebook for the phase of the non-reference channel fading coefficients is shown in Fig. 7.14b. For the sake of clarity, the superscript $(n)$, which denotes the $n$th non-reference fading, has been omitted in the magnitude and phase of the channel fading coefficients and in the parameters of the quantizer, $\left(d_{r 0}, d_{r}\right.$ and $\left.d_{\Delta}\right)$, since a different quantizer has to be obtained for each non-reference fading. However, the subindex $n$ has been kept in the number of quantization levels and in the correlation coefficient as this will be needed in the study of the overall distortion and bit allocation. The partitions and the output values of the quantizer of the non-reference channel fading coefficients can be expressed as:

$$
\begin{array}{rlrl}
r_{\mathrm{nr}, m} & =d_{r 0}+m d_{r} & m & =1, \ldots, M_{\mathrm{nr}, n}-1 \\
\hat{r}_{\mathrm{nr}, m} & =d_{r 0}+(m-1 / 2) d_{r} & m & =1, \ldots, M_{\mathrm{nr}, n} \\
\Delta_{p} & =p d_{\Delta} & p & =-\left(\frac{P_{\mathrm{nr}, n}}{2}-1\right), \ldots,\left(\frac{P_{\mathrm{nr}, n}}{2}-1\right) \\
\hat{\Delta}_{p} & =(p-1 / 2) d_{\Delta} & p & =-\left(\frac{P_{\mathrm{nr}, n}}{2}-1\right), \ldots, \frac{P_{\mathrm{nr}, n}}{2}
\end{array}
$$

where $M_{\mathrm{nr}, n}$ and $P_{\mathrm{nr}, n}$ are the number of quantization levels for the envelope and phase of the $n$th non-reference channel fading, respectively, and $m$ and $p$ are integers.

For fixed values of the number of quantization levels for envelope and phase, and omitting the quantization error of the reference fading, the distortion function for the $n$th non-reference channel fading, given a certain reference envelope $r_{\mathrm{r}}$ and the correlation coefficient between the $n$th non-reference channel fading and the reference one, $\rho_{n}$, can be expressed as

$$
\begin{aligned}
& D_{\mathrm{nr}}\left(d_{r 0}, d_{r}, d_{\Delta}, \rho_{n} \mid r_{\mathrm{r}}\right)= \\
& \quad \sum_{m=1}^{M_{\mathrm{nr}, n}} \sum_{p=1}^{P_{\mathrm{nr}, n}} \int_{r_{\mathrm{nr}, m-1}}^{r_{\mathrm{nr}, m}} \int_{\Delta_{p-1}}^{\Delta_{p}}\left|r_{\mathrm{nr}} e^{j \Delta}-\hat{r}_{\mathrm{nr}, m} e^{j \hat{\Delta}_{p}}\right|^{2} f\left(r_{\mathrm{nr}} \mid r_{\mathrm{r}}, \rho_{n}\right) f_{\Delta}\left(\Delta, \rho_{n}\right) \mathrm{d} r_{\mathrm{nr}} \mathrm{d} \Delta,
\end{aligned}
$$

where $\Delta=\theta_{\mathrm{nr}}-\left(\hat{\theta}_{\mathrm{r}}-\theta_{\rho_{n}}\right)$ is the phase deviation. Likewise, $M_{\mathrm{nr}, n}$ and $P_{\mathrm{nr}, n}$ are considered to be power of 2 integers. Thus, $B_{M_{\mathrm{nr}, n}}=\log _{2} M_{\mathrm{nr}, n}, B_{P_{\mathrm{nr}, n}}=\log _{2} P_{\mathrm{nr}, n}$ and $B_{\mathrm{nr}, n}=B_{M_{\mathrm{nr}, n}}+B_{P_{\mathrm{nr}, n}}$ are the number of bits dedicated to quantizing the envelope, the phase and the total number of bits for the $n$th non-reference channel fading, respectively. 


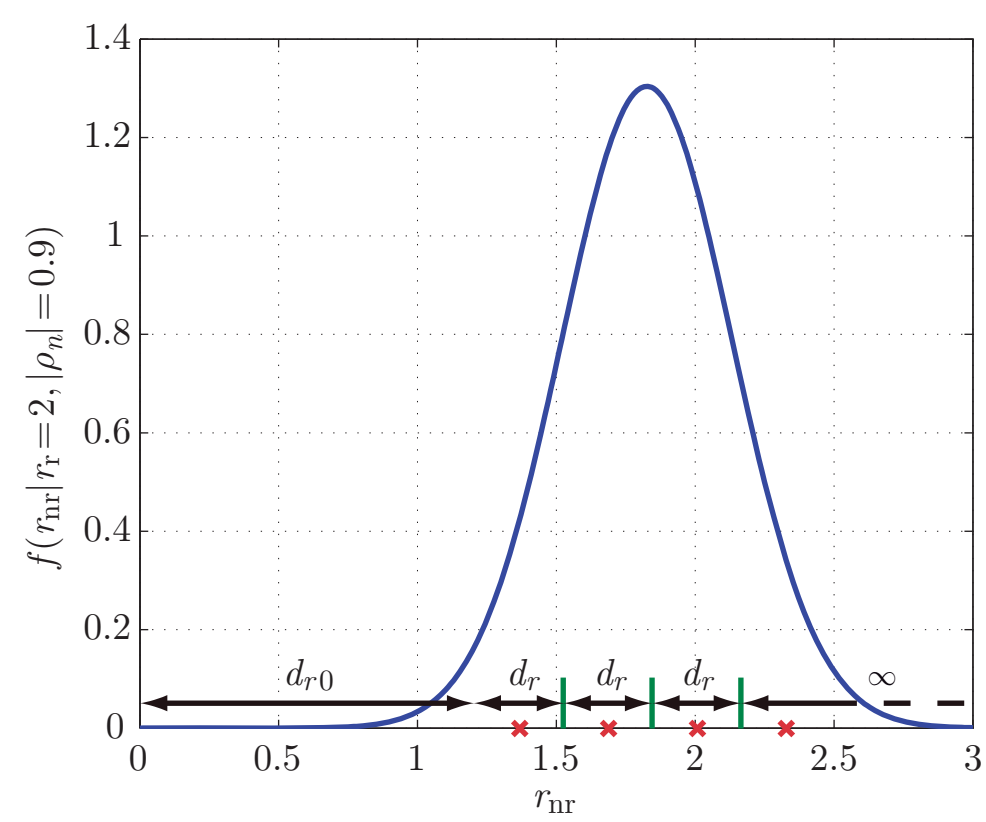

(a) Codebook for envelope quantization

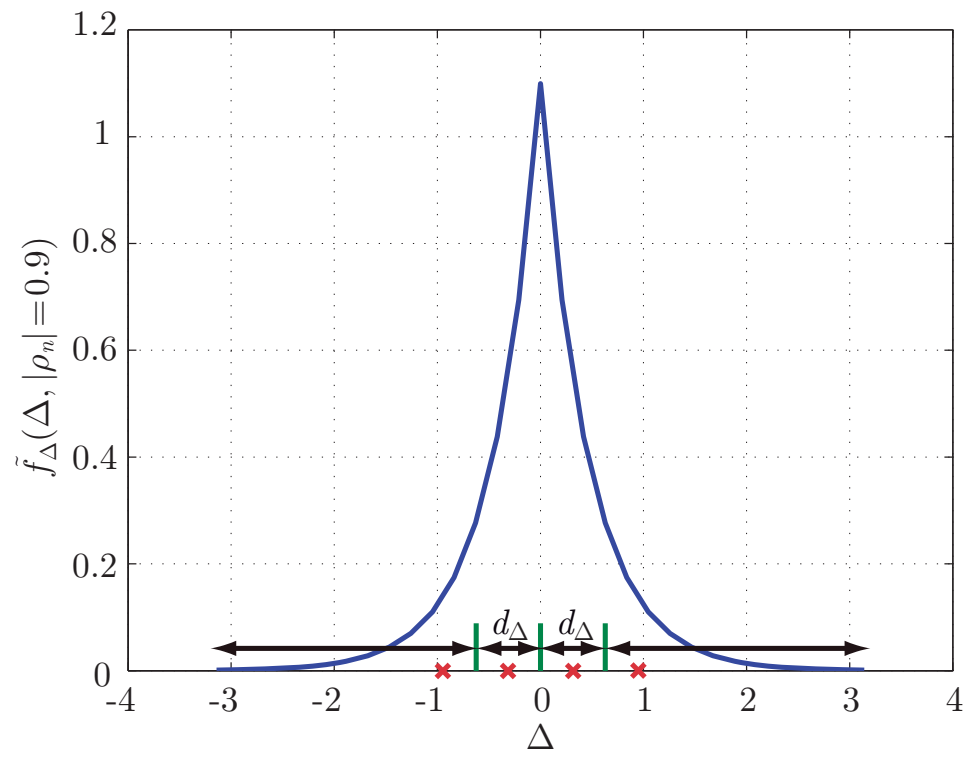

(b) Codebook for phase quantization

Figure 7.14: Illustrative example of parameters $d_{r 0}, d_{r}$ and $d_{\Delta}$ for a non-reference codebook for $r_{\mathrm{r}}=2,\left|\rho_{n}\right|=0.9, M_{\mathrm{nr}, n}=4$ and $P_{\mathrm{nr}, n}=4$. The partitions and the output values are represented by green segments and red $\mathrm{X}$ marks, respectively. 
The solution for the definite integrals in (7.43) that involve the PDF of the phase deviation, $f_{\Delta}\left(\Delta, \rho_{n}\right)$, requires numerical integration and the evaluation of the Gaussian hypergeometric function, ${ }_{2} F_{1}(\cdot, \cdot ; \cdot ; \cdot)$, which results in a significant computational cost. In addition, the minimization algorithm may require multiple evaluations of the distortion function at different points to obtain the optimal codebook for each non-reference channel fading. In order to avoid this highly demanding computation, an approximation to (7.32) is presented using a truncated Laplace distribution:

$$
\tilde{f}_{\Delta}\left(\Delta, \rho_{n}\right)= \begin{cases}\frac{\exp \left(-|\Delta| / b_{\mathrm{L}, n}\right)}{2 b_{\mathrm{L}, n}\left(1-\exp \left(-\pi / b_{\mathrm{L}, n}\right)\right)}, & -\pi \leq \Delta<\pi \\ 0, & \text { otherwise }\end{cases}
$$

where $b_{\mathrm{L}, n}$ is the parameter of the Laplace distribution that can be accurately adjusted in terms of least square fitting for high correlation environments by

$$
b_{\mathrm{L}, n}^{2}=0.52\left|\rho_{n}\right|^{2}-2.96\left|\rho_{n}\right|+2.45 .
$$

This approximation allows the definite integral in (7.43) to be solved analytically using the results provided in the Appendix B.

Figure 7.15 shows the analytical PDF of the phase deviation, seen in (7.32), and the proposed Laplace approximation, seen in (7.44), for different values of $\rho_{n}$. It can be observed that, as the correlation coefficient increases, the approximation is closer to the analytical PDF and it includes a higher error for small magnitudes of the correlation coefficient.

Using the proposed approximation (7.44) in (7.43), the distortion for the $n$th nonreference channel fading given a certain reference envelope, $r_{\mathrm{r}}$, and the correlation coefficient between the $n$th non-reference antenna and the reference antenna, $\rho_{n}$, can be expressed as

$$
\begin{aligned}
& D_{\mathrm{nr}}\left(d_{r 0}, d_{r}, d_{\Delta}, \rho_{n} \mid r_{\mathrm{r}}\right)= \\
& \sum_{m=1}^{M_{\mathrm{nr}, n}} \int_{r_{\mathrm{nr}, m-1}}^{r_{\mathrm{nr}, m}} r_{\mathrm{nr}}^{2} f\left(r_{\mathrm{nr}} \mid r_{\mathrm{r}}, \rho_{n}\right) \mathrm{d} r_{\mathrm{nr}}+\sum_{m=1}^{M_{\mathrm{nr}, n}} \hat{r}_{\mathrm{nr}, m}^{2} \int_{r_{\mathrm{nr}, m-1}}^{r_{\mathrm{nr}, m}} f\left(r_{\mathrm{nr}} \mid r_{\mathrm{r}}, \rho_{n}\right) \mathrm{d} r_{\mathrm{nr}} \quad \text { (7.46) } \\
& -2 \sum_{m=1}^{M_{\mathrm{nr}, n}} \hat{r}_{\mathrm{nr}, m} \int_{r_{\mathrm{nr}, m-1}}^{r_{\mathrm{nr}, m}} r_{\mathrm{nr}} f\left(r_{\mathrm{nr}} \mid r_{\mathrm{r}}, \rho_{n}\right) \mathrm{d} r_{\mathrm{nr}} \sum_{p=1}^{P_{\mathrm{nr}, n}} \int_{\Delta_{p-1}}^{\Delta_{p}} \cos \left(\Delta-\hat{\Delta}_{p}\right) \tilde{f}_{\Delta}\left(\Delta, \rho_{n}\right) \mathrm{d} \Delta,
\end{aligned}
$$

where the first three integrals can be solved using the 2nd, 0th and 1st moments of $f\left(r_{\mathrm{nr}} \mid r_{\mathrm{r}}, \rho_{n}\right)$, respectively, as shown in (7.27), and the solution of the fourth integral can be found in equation (B.17) of the Appendix B.

The convergence of the minimization of the distortion expressed in (7.46), which is a 3 -dimension minimization problem on $\left(d_{r 0}, d_{r}, d_{\Delta}\right)$, is not straightforward to analyze and is out of the scope of this paper. The Nelder-Mead simplex method 


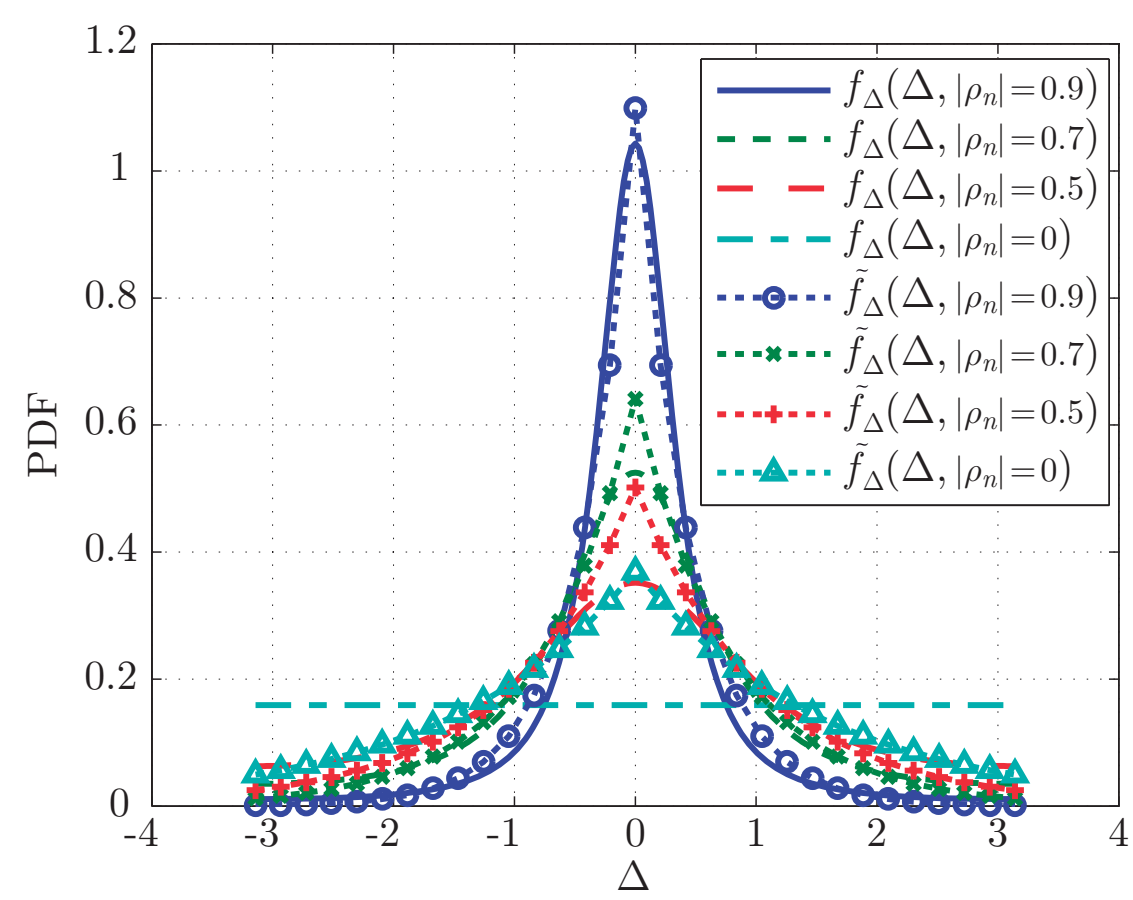

Figure 7.15: Analytical PDF of the phase deviation, $f_{\Delta}\left(\Delta, \rho_{n}\right)$, and its approximation using the Laplace distribution, $\tilde{f}_{\Delta}\left(\Delta, \rho_{n}\right)$, for different values of the correlation coefficient, $\left|\rho_{n}\right|$.

can be used to minimize the distortion due to the good convergence properties that it has demonstrated in other problems and because it does not require any derivative information [289].

After carrying out preliminary simulations using non-uniform codebooks, the MSE obtained has been slightly lower. Nevertheless, the convergence criterion of the minimization problem for non-uniform codebooks was not met in all cases. For this reason, only the results obtained with uniform codebooks have been included, where the convergence was achieved in all cases. 


\section{Overall distortion}

In order to express the overall distortion, it is necessary to determine both the distortion due to the quantization of the reference channel fading and the distortion due to the quantization of the non-reference channel fading coefficients. In what follows, it is assumed that the aforementioned quantizers are always optimized for the different number of levels and the distortion is expressed in terms of the number of quantization bits. Thus, the distortion in the reference and non-reference fading quantization, seen in (7.38) and (7.43), for different number of quantization bits is denoted as $D_{\mathrm{r}}\left(B_{M_{\mathrm{r}}}, B_{P_{\mathrm{r}}}\right)$ and $D_{\mathrm{nr}}\left(B_{M_{\mathrm{nr}, n}}, B_{P_{\mathrm{nr}, n}}, \rho_{n} \mid r_{\mathrm{r}}\right)$, respectively.

The distortion in a non-reference channel fading has been expressed in (7.43) and depends on the current reference channel fading and the correlation coefficient. The average distortion in the $n$th non-reference channel fading for a given correlation coefficient can be expressed as

$$
D_{\mathrm{nr}}\left(B_{M_{\mathrm{nr}, n}}, B_{P_{\mathrm{nr}, n}}, \rho_{n}\right)=\sum_{m=1}^{M_{\mathrm{r}}} D_{\mathrm{nr}}\left(B_{M_{\mathrm{nr}, n}}, B_{P_{\mathrm{nr}, n}}, \rho_{n} \mid \hat{r}_{\mathrm{r}, m}\right) P\left(\hat{r}_{\mathrm{r}, m}\right),
$$

where $\hat{r}_{\mathrm{r}, m}$ is the $m$ th value in the codebook of the reference envelope and $P\left(\hat{r}_{\mathrm{r}, m}\right)$ is the probability of quantizing a reference envelope with the value $\hat{r}_{\mathrm{r}, m}$, (i.e., the probability of the $m$ th envelope decision interval). This probability is given by

$$
P\left(\hat{r}_{\mathrm{r}, m}\right)=\int_{r_{\mathrm{r}, m-1}}^{r_{\mathrm{r}, m}} f\left(r_{\mathrm{r}}\right) \mathrm{d} r_{\mathrm{r}}=\Gamma\left(1, \frac{r_{\mathrm{r}, m-1}^{2}}{2 b_{\mathrm{R}}^{2}}\right)-\Gamma\left(1, \frac{r_{\mathrm{r}, m}^{2}}{2 b_{\mathrm{R}}^{2}}\right),
$$

where $\Gamma(s, x)$ denotes the upper incomplete Gamma function [286, Sec. 6]. Thus, the distortion over the whole channel vector can be expressed as

$$
D\left(B_{M_{\mathrm{r}}}, B_{P_{\mathrm{r}}}, \mathbf{b}_{M_{\mathrm{nr}}}, \mathbf{b}_{P_{\mathrm{nr}}}, \boldsymbol{\rho}\right)=D_{\mathrm{r}}\left(B_{M_{\mathrm{r}}}, B_{P_{\mathrm{r}}}\right)+\sum_{n \in \mathcal{S}_{\mathrm{nr}}} D_{\mathrm{nr}}\left(B_{M_{\mathrm{nr}, n}}, B_{\left.P_{\mathrm{nr}, n}, \rho_{n}\right),}\right.
$$

where $\mathbf{b}_{M_{\mathrm{nr}}}, \mathbf{b}_{P_{\mathrm{nr}}}$ and $\boldsymbol{\rho}$ are vectors that contain $B_{M_{\mathrm{nr}, n}}, B_{P_{\mathrm{nr}, n}}$ and $\rho_{n}$ for the $N_{t}-1$ non-reference channel fading coefficients and $\mathcal{S}_{\mathrm{nr}}$ is the set of the $N_{t}-1$ non-reference antennas. The number of quantization bits dedicated to quantizing the non-reference fading coefficients is given by $B_{\mathrm{nr}}=\sum_{n \in \mathcal{S}_{\mathrm{nr}}} B_{\mathrm{nr}, n}$, and the total number of quantization bits is $B=B_{\mathrm{r}}+B_{\mathrm{nr}}$. 


\section{Bit allocation}

The previous subsections have shown how to obtain the optimal codebook that minimizes the distortion in reference and non-reference channel fading. However, the solution for allocating the total number of bits to quantize the envelopes and phases of the reference and non-reference channel fading coefficients has not been discussed yet.

The number of bits dedicated to quantizing the reference channel fading depends on the correlation between the channel fading coefficients. For uncorrelated or low-correlated environments, the best performance is obtained using an equal bit allocation per channel fading since the PDFs of the different channel fading coefficients are almost equal. However, as the correlation increases, a lower overall distortion can be achieved by increasing the number of bits of the reference channel fading, since this value will be used for the quantization of non-reference fading coefficients and the variance of the rest of channel fading coefficients will be lower. In the proposed scheme, the number of quantization bits for the reference channel fading varies from $B_{\mathrm{r}}=\left\lceil B / N_{t}\right\rceil$ to $B_{\mathrm{r}}=\left\lceil B / N_{t}\right\rceil+3$, depending on the correlation. Therefore, the optimal bit allocation is obtained from pre-calculated tables.

For the envelope and phase quantization of the reference channel fading, the average ratio between the number of levels of the phase and the magnitude quantizers for the minimum distortion is 2.77 [288], which is equivalent to allocating 1.47 more bits to the phase than to the magnitude. Since the number of levels is assumed to be power of 2 integers, this condition can be obtained by ensuring that

$$
\left(B_{M_{\mathrm{r}}}, B_{P_{\mathrm{r}}}\right): \begin{cases}B_{P_{\mathrm{r}}}-B_{M_{\mathrm{r}}}=1 & B_{\mathrm{r}} \text { odd } \\ B_{P_{\mathrm{r}}}-B_{M_{\mathrm{r}}}=2 & B_{\mathrm{r}} \text { even }\end{cases}
$$

This technique has proven to obtain the minimum distortion for integer bit allocation in at least the range $3 \leq B_{\mathrm{r}} \leq 14$. Therefore, the number of bits dedicated to the reference envelope and phase can be calculated directly as follows

$$
\begin{aligned}
& B_{M_{\mathrm{r}}}=\left\lceil\frac{B_{\mathrm{r}}}{2}\right\rceil-1 \\
& B_{P_{\mathrm{r}}}=\left\lfloor\frac{B_{\mathrm{r}}}{2}\right\rfloor+1 .
\end{aligned}
$$

With regard to the bit allocation between the non-reference antennas, $B_{\mathrm{nr}}$ bits have to be allocated between the envelope and the phase of the non-reference fading coefficients, $B_{M_{\mathrm{nr}, n}}$ and $B_{P_{\mathrm{nr}, n}}$ bits respectively, in order to minimize (7.49). The GBA shown in Algorithm 5 is used to perform this task considering the distortion given in (7.47). Results show that the overall distortion is minimized by dedicating more bits to those channel fading coefficients that exhibit a lower correlation with 


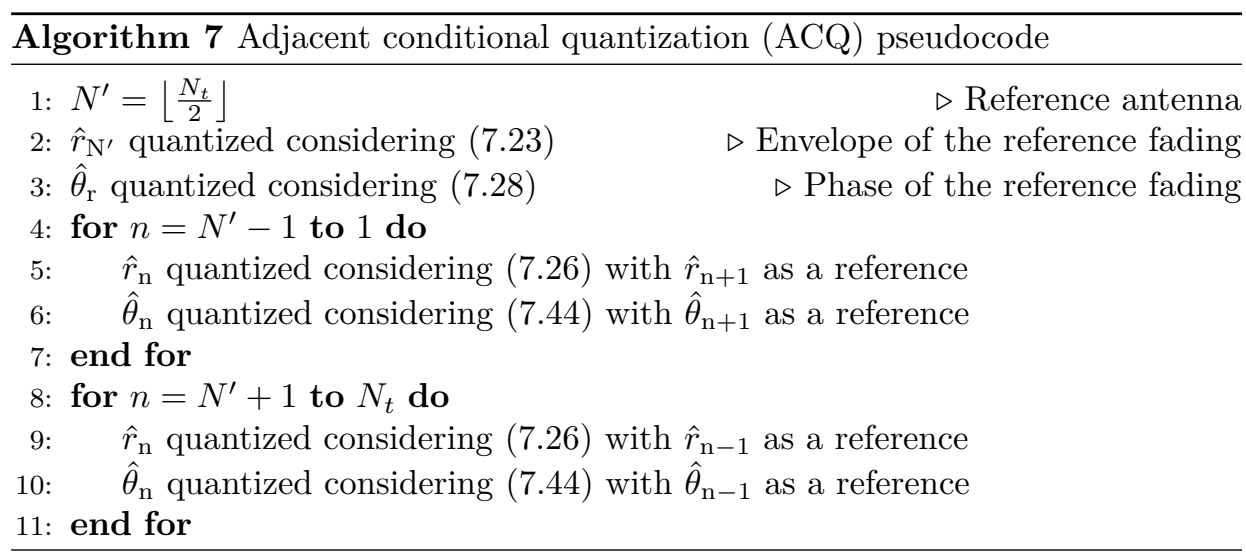

the reference one, which in a linear array would be those fading coefficients whose antennas are located the farthest away from the reference one.

\subsubsection{Alternative quantization scheme}

The main disadvantage of the CQ scheme presented in the previous section and in $[176]$ is that the correlation between the reference antenna and the antennas located at the edges of the array can be too small in large arrays or in arrays with a large antenna separation, $d$. In order to mitigate this issue, an alternative quantization scheme based on the same statistical characterization is presented in this section.

The main idea of this new scheme is that the channel fading coefficient from a given antenna is quantized taking as a reference the fading coefficient from the adjacent antenna. Thus, the first step of this quantization scheme is to select one of the central antennas as a reference. In uniform linear arrays, the reference antenna will probably fulfill (7.33), as in the case of the CQ scheme. Since there is no reference to quantize this channel fading coefficient, its envelope and phase are quantized considering (7.23) and (7.28), respectively, and using uniform polar quantization. Next, the fading coefficients from the two adjacent antennas are quantized considering (7.26) and (7.44) for the envelope and phase, respectively, as the GQ scheme. Next, the fading coefficient of the antenna separated $2 d$ from the reference antenna is quantized, but considering the adjacent antenna (separated $d$ from it) as the reference one. Then, the antenna separated $3 d$ from the reference antenna is quantized based on the fading of the antenna separated $2 d$, and so on. This scheme will be referred to as adjacent conditional quantization (ACQ). The different steps and the pseudocode of the ACQ scheme have been detailed in Fig. 7.16 and Algorithm 7. 


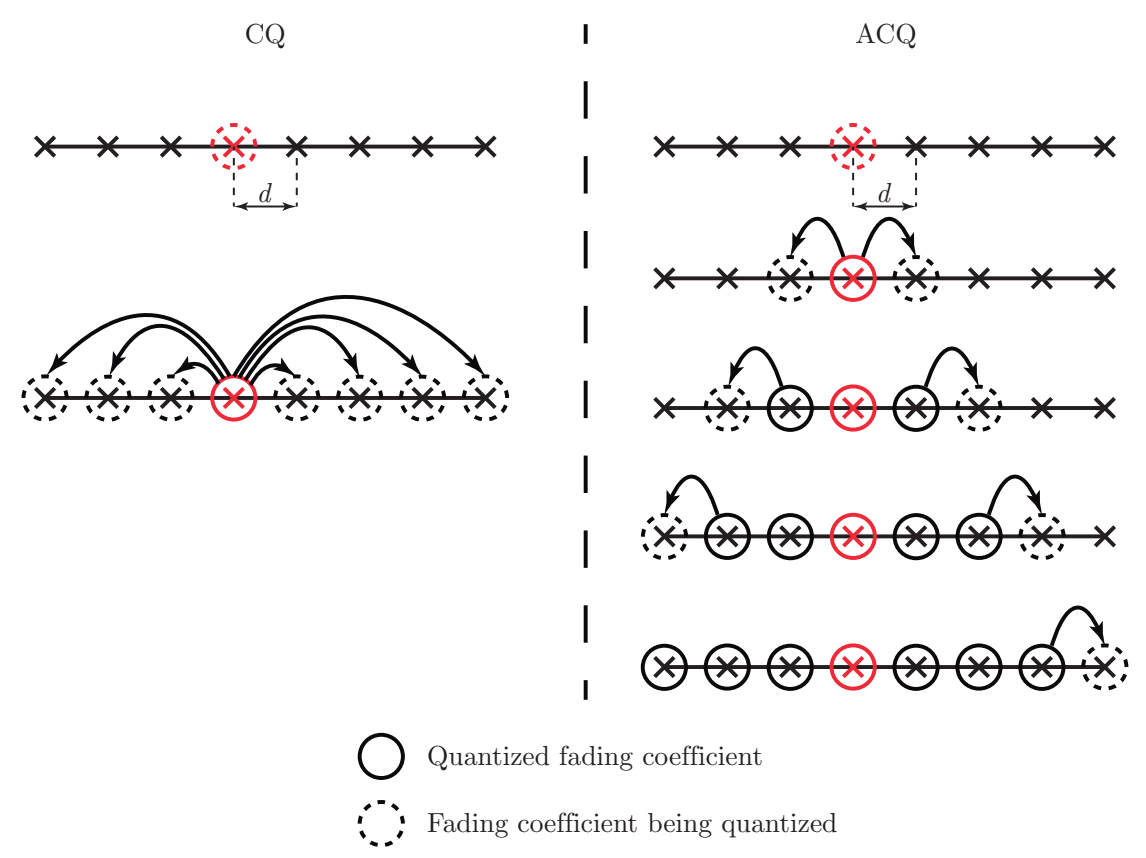

Figure 7.16: Comparison between $\mathrm{CQ}$ and ACQ.

Fig. 7.16 shows the difference between the ACQ scheme and the CQ scheme. In the figure, arrows have been drawn from the reference fading coefficients to the fading coefficients that are being quantized at each step. The main advantage of the ACQ over CQ is that the reference fading is always the one from the adjacent antenna, entailing a higher correlation between fading coefficients. This leads to PDFs with lower variances and lower quantization errors. Another advantage of $\mathrm{ACQ}$ is that the bit allocation task is easier. Since the correlation between each pair of adjacent antennas is usually the same, the total number of bits can be equally distributed between the non-reference fading coefficients.

\subsubsection{Numerical results}

This section evaluates the performance of the proposed quantization schemes. To the best of our knowledge, no previous work has proposed different PDFs that are based on the correlation coefficient in order to quantize the different channel fading coefficients. Thus, the proposed schemes are compared with a scheme that is based on PQ where identical PDFs are used for all the channel fading coefficients since that scheme does not consider correlation [288], and with another scheme that is based on the KL transform [259, 290]. The GBA is used in both of these schemes [259]. 
As stated previously, the KL scheme requires the knowledge of the entire spatial correlation matrix at the BS. Since the spatial correlation matrix exhibits a Hermitian symmetry with a unit diagonal [291], it can be reconstructed at the BS by sending back the coefficients above or below the main diagonal. Therefore, the number of coefficients of the spatial correlation matrix that need to be estimated and sent back in the KL scheme is given by

$$
N_{\mathrm{c}, \mathrm{KL}}=\frac{N_{t}\left(N_{t}-1\right)}{2},
$$

and it increases with $N_{t}^{2}$. The CQ scheme only requires the correlation coefficients between the reference channel fading and the rest of the channel fading coefficients, that is,

$$
N_{\mathrm{c}, \mathrm{CQ}}=N_{t}-1 \text {, }
$$

resulting in a linear relation between the number of coefficients and the number of antennas. With regard to the ACQ scheme, correlation coefficients for the different pairs of adjacent antennas are very close in practice and even the same in many correlation models such as in [285]. In this case, only one correlation coefficient would be required. However, the scheme will be generalized for non-uniform arrays and will consider the same number of correlation coefficients as the CQ scheme,

$$
N_{\mathrm{c}, \mathrm{ACQ}}=N_{t}-1
$$

However, it is important to note that this fact could be leveraged in order to further improve the performance of ACQ. Thus, the KL scheme needs to estimate and provide $N_{t} / 2$ times the number of correlation coefficients sent back by the CQ and ACQ scheme. Since the MSs have different spatial correlation matrices depending on their location, this information has to be fed back by every MS. In contrast, the PQ scheme does not require any correlation coefficient.

MSs must keep the spatial correlation information periodically updated. The periodicity parameter $N_{\mathrm{pd}}$ (in subframes) shows how often this information is updated in the LTE-A standard [292]. Since one CSI reference signal is sent every subframe [11, Chap. 29], the parameter $L_{\mathrm{ch}}=N_{\mathrm{pd}}$ is defined as the number of channel quantizations that can be carried out before updating the correlation information. Note that the quantization of the spatial correlation matrix entails a reduction of the feedback rate dedicated to quantizing the instantaneous channel information in systems with a fixed feedback rate.

With regard to the computational complexity of the CQ, ACQ and KL schemes, the CQ and ACQ schemes directly quantize the envelope and phase of the channel fading coefficients, while the KL must perform a KL transform, and subsequently quantize the resulting KL coefficients. On the other hand, the CQ and ACQ schemes require the calculation of an independent codebook for each value of the correlation coefficient and each quantized value of the envelope of the reference 


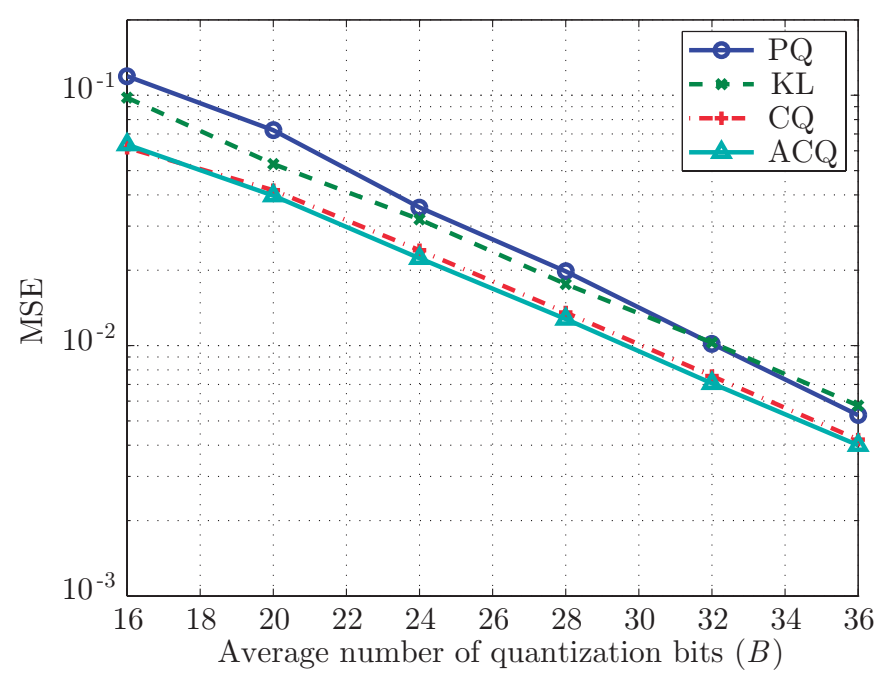

Figure 7.17: MSE for $N_{t}=4, d=\lambda / 2$ and different average number of quantization bits.

channel fading, while the KL scheme simply scales a Gaussian codebook for quantizing each KL coefficient. However, the symmetry around the reference antenna in uniform linear arrays allows the CQ codebook for one antenna to be generated from its symmetric counterpart. In addition, the ACQ only requires the calculation of one codebook if the correlation between each pair of adjacent antennas is the same in uniform arrays. Codebooks in the CQ and ACQ schemes can be stored in tables based on the tabulated correlation coefficient so that channel fading coefficients can be directly quantized from these tables.

In the simulation, the suburban macrocell scenario of the SCM has been considered [182]. The system consists of a BS equipped with $N_{t}$ antennas, an adjacent antenna separation of $d$ meters, several single antenna MSs moving at $v_{\mathrm{MS}}=40$ $\mathrm{km} / \mathrm{h}$ and a carrier frequency of $f_{c}=2 \mathrm{GHz}$. The effects of path loss and shadowing have not been considered. Since the SCM does not specify the spatial correlation explicitly, the correlation estimation has been performed using the maximum likelihood estimator over segments of $40 \mathrm{~ms}$, as detailed in [293]. The parameter $L_{\mathrm{ch}}$ is set to 10 , which is one of the possible values detailed in [292]. Uniform quantization is used to quantize the correlation coefficients using $B_{\mathrm{cc}}=5$ bits, which is a reasonable value according to [294].

Figures 7.17-7.19 compare the performance of the PQ, KL, CQ and ACQ schemes regarding the estimated MSE per antenna, obtained through Monte Carlo simulations for different number of quantization bits. The estimated MSE per antenna 
can be expressed as

$$
\mathrm{MSE} \approx \frac{1}{K N_{t}} \sum_{k=1}^{K}\left\|\mathbf{h}_{k}-\hat{\mathbf{h}}_{k}\right\|^{2},
$$

where $K=10^{5}$ is the number of channel realizations in the simulation and $\mathbf{h}_{k}$ and $\hat{\mathbf{h}}_{k}$ are the original and quantized channels, respectively, for the $k$ th channel realization. While the PQ scheme uses all the bits to quantize the instantaneous CSI, the KL, CQ and ACQ schemes dedicate some bits to quantize the correlation coefficients. In order to make a fair comparison, the sum of bits dedicated to quantizing the instantaneous CSI and those dedicated to quantizing the correlation information during $L_{\mathrm{ch}}$ quantization processes is the same for the four schemes. Thus, the horizontal axis in the three figures shows the average number of bits per channel quantization.

Figure 7.17 shows the MSE performance in a highly correlated scenario where the separation between two adjacent antennas of the array is set to $d=\lambda / 2$. It can be observed that the CQ and ACQ schemes outperform the PQ and KL schemes, allowing a reduction of about $2-4$ quantization bits for a given MSE. The performance of the CQ and ACQ schemes are almost the same because the distance between the reference antenna in the $\mathrm{CQ}$ scheme and the antennas at the edge of the array is not large. Although the KL scheme is considered to be optimal in terms of decorrelation of Gaussian sources, it quantizes more correlation coefficients than the proposed schemes as seen in (7.53)-(7.55), which in turn results in fewer bits available to quantize the instantaneous CSI. In addition, the KL scheme presents a higher sensitivity to estimation and quantization errors in the correlation coefficients than the CQ scheme. This drawback could be solved by increasing $B_{\mathrm{cc}}$, but it entails a reduction in the number of bits dedicated to quantizing the instantaneous CSI, obtaining a similar performance (results with $B_{\mathrm{cc}}=8$ are omitted since a very similar performance to that shown for $B_{\mathrm{cc}}=5$ is obtained). The choice for the optimal $B_{\mathrm{cc}}$ may depend on the value of $L_{\mathrm{ch}}$, since a longer validity period can be advantageous in order to provide more accurate correlation information, but this analysis is out of the scope of this thesis.

In Fig. 7.18, the adjacent antenna separation is set to $d=\lambda$. Consequently, the correlation between the transmit antennas decreases as does the difference between the performance of the CQ scheme and the PQ scheme. However, the CQ scheme still outperforms the PQ scheme in almost the entire considered range. The improvement of the ACQ scheme over CQ is due to the increase in the distance between reference and non-reference antennas in the CQ scheme. Finally, the poor performance obtained by the KL scheme can be explained by the considerable number of feedback bits that are used to quantize the correlation coefficients, whereas the correlation is not as high in Fig. 7.18 as in Fig. 7.17.

Figure 7.19 shows the MSE performance for a system with $N_{t}=8$ transmit antennas and $d=\lambda / 2$. It is important to note that, even though the antenna separation 


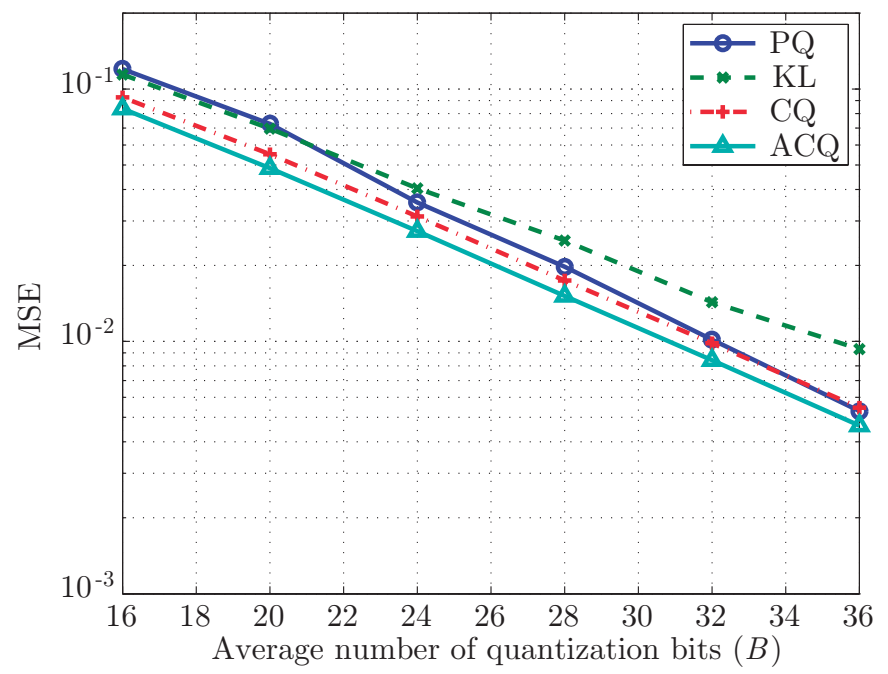

Figure 7.18: MSE for $N_{t}=4, d=\lambda$ and different average number of quantization bits.

is as small as in Fig. 7.17, the distance between non-adjacent antennas increases with the number of antennas, and the correlation between non-adjacent antennas decreases. In this case, the CQ scheme outperforms the PQ and KL schemes, but the differences between the PQ scheme and the CQ scheme are not as relevant. However, the ACQ scheme offers a considerable improvement over the rest of schemes. Unlike the CQ scheme, the ACQ is barely affected by the increase in the number of antennas. The KL scheme has to quantize $N_{\mathrm{c}, \mathrm{KL}}=28$ correlation coefficients whereas $\mathrm{CQ}$ and $\mathrm{ACQ}$ only need to quantize $N_{\mathrm{c}, \mathrm{CQ}}=N_{\mathrm{c}, \mathrm{ACQ}}=7$. Thus, CQ and ACQ can use more bits to quantize the instantaneous CSI and performs better over the entire range of bits.

Figure 7.20 shows the MSE of the different schemes in terms of the adjacent antenna separation, $d$, for $N_{t}=4$ antennas and an average number of 28 quantization bits, whereas 7.21 shows the MSE for $N_{t}=8$ antennas and an average number of 56 quantization bits. Note that spatial correlation together with the statistical characterization of the respective fading can be successfully used to reduce the MSE in the quantization of a MIMO channel in highly correlated environments, corresponding to arrays with close antennas. In the simulated systems, the correlation could be leveraged by the CQ scheme to obtain the best performance for antenna separations below $d=1.3 \lambda$ and $d=0.75 \lambda$ for the cases of $N_{t}=4$ and $N_{t}=8$ antennas, respectively. In the ACQ scheme, the correlation could be exploited for antenna separations below around $d=1.5 \lambda$ for both cases, showing that the number of antennas barely affects this scheme. It is important to note that the CQ and ACQ schemes are equivalent to the PQ scheme when the correlation is not considered, although this case has not been contemplated in 


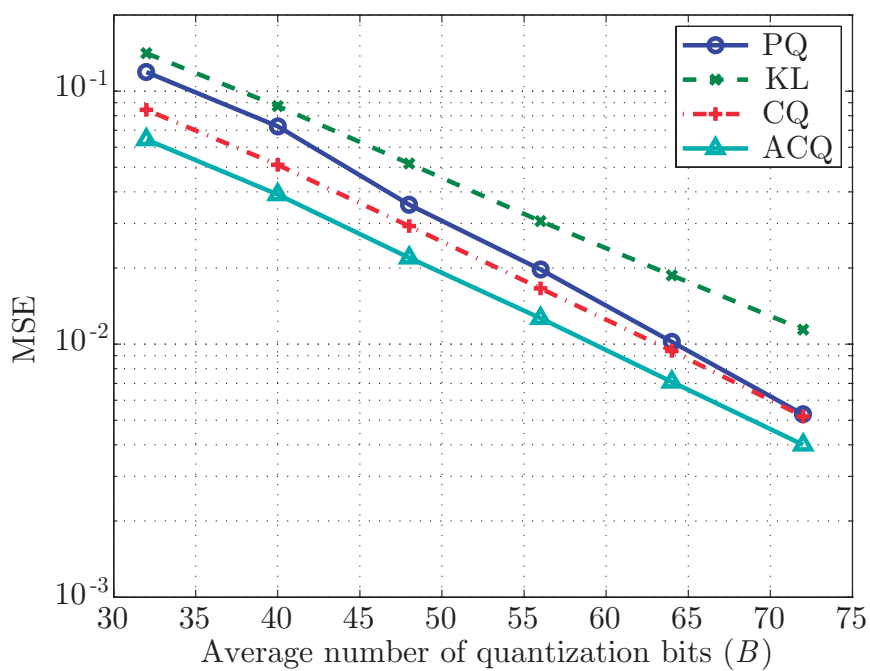

Figure 7.19: MSE for $N_{t}=8, d=\lambda / 2$ and different average number of quantization bits.

the simulations. Thus, the MSs would hypothetically choose whether or not to provide the correlation information, assuming the BS that $\rho=0$ when it is not provided, and achieving the best performance between CQ/ACQ and PQ for each case. Similarly, the KL scheme is equivalent to rectangular quantization when the correlation is not considered and its performance is almost identical to the PQ scheme [261].

\subsubsection{Conclusion}

In this section, the Kronecker correlation model has been considered and a spatial statistical characterization of the channel for a system with multiple transmit antennas has been presented. In this characterization, one antenna is selected as the reference antenna and the channel fading from this antenna is considered to be the reference channel fading. The statistics for the fading of the rest of the channels are stated and related to the reference fading by means of the correlation coefficient. The envelope of a non-reference channel fading is characterized using the CPDF given the envelope of the reference channel fading and the correlation coefficient of the non-reference channel fading with the reference fading. The phase of a non-reference channel fading is characterized by using an approximation based on high correlation and the statistical characterization of the error induced by this approximation. 


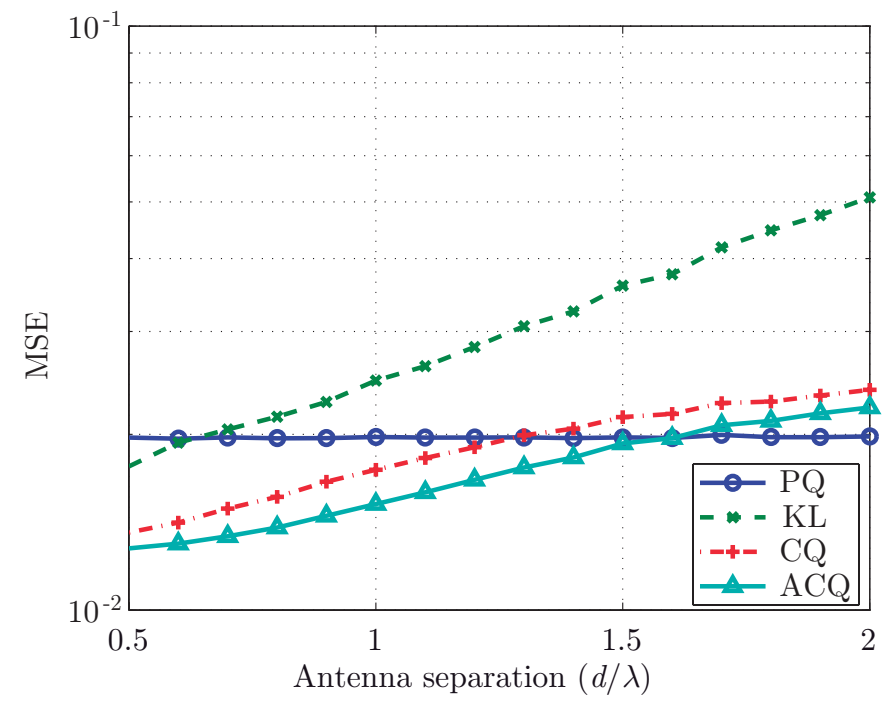

Figure 7.20: MSE for $N_{t}=4$, different antenna separations and an average number of quantization bits of 28 bits.

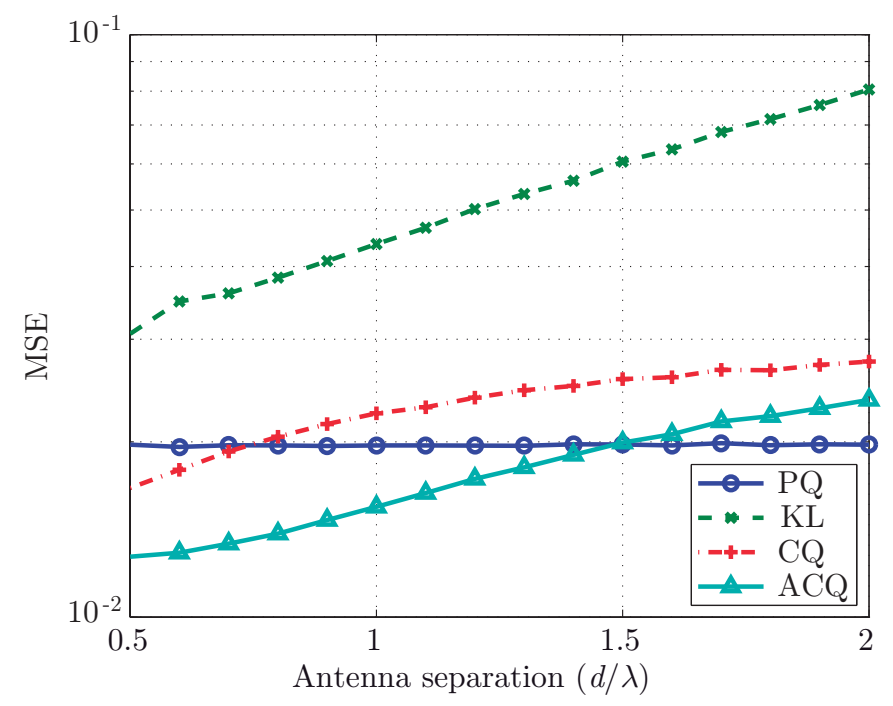

Figure 7.21: MSE for $N_{t}=8$, different antenna separations and an average number of quantization bits of 56 bits.

Two channel quantization schemes that make use of this characterization to reduce the feedback information has also been proposed. In these schemes, the envelope 
and the phase of the reference channel fading are quantized considering a Rayleigh distribution and a uniform distribution, respectively. The envelopes of the nonreference channel fading coefficients are quantized according to their conditional PDF given the reference channel fading envelope and the correlation coefficient. Finally, the phase of the non-reference channel fading is fed back by quantizing the error of its approximation using the proposed characterization. The difference between these schemes is that the reference channel fading is fixed for the CQ scheme, whereas the ACQ always takes the adjacent channel fading as a reference.

The proposed schemes have been compared with another scheme based on standard polar quantization, where the spatial correlation is not taken into account, and with a scheme based on the KL transform, that achieves maximum decorrelation of Gaussian sources. The main advantage of the proposed schemes is that, unlike the scheme based on the KL transform, they do not require the entire spatial correlation matrix. Thus, fewer feedback bits are used to quantize the correlation information and more bits are available to quantize the instantaneous CSI. The comparison has been carried out using the SCM. Numerical results show that the proposed scheme clearly outperforms the other two schemes in highly correlated scenarios. For the particular parameters used in the simulations, the CQ scheme allows the spatial correlation to be successfully leveraged in arrays with antenna separations that are lower than $d=1.3 \lambda$ and $d=0.75 \lambda$ for $N_{t}=4$ and $N_{t}=8$ antennas, respectively. In the case of the ACQ scheme, results show that this scheme is capable of successfully exploiting the spatial correlation for antenna separations that are below $d=1.5 \lambda$, independently of the number of antennas. In addition, these schemes can be straightforwardly adapted to ignore the correlation when no benefit is obtained from it.

\subsection{Conclusion}

This chapter has presented the spatial statistical characterization of the SCM and the Kronecker correlation model. Based on these characterizations, three different channel quantization schemes have been presented.

First, the spatial statistical characterization of the SCM has been presented. Due to the large number of parameters and the complexity of the model, the characterization has been carried out only for the high correlation case. In addition, some approximations have been required in order to use the characterization in the proposed quantization scheme. Results have shown that the proposed scheme clearly outperforms the scheme based on GQ in highly correlated environments.

In order to obtain a more complete spatial statistical characterization, the Kronecker correlation model has been considered. This correlation model is simpler and allows obtaining the characterization for high and low correlations. Based on 
this characterization, two channel quantization schemes have been presented an evaluated using the SCM. Results show that the proposed schemes outperforms some existing schemes in highly and moderately correlated scenarios. 


\section{Chapter 8}

\section{Conclusions}

MIMO communication systems have emerged as one of the most promising technologies that allow exploiting the spatial dimension in addition to the time and frequency dimensions. MIMO techniques can also be used in a multiuser scenario, where several users share the spatial dimension causing multiuser interference. The use of precoding allows the mitigation of multiuser interference even in systems with single-antenna receivers.

However, these benefits come at the expense of a more complex system. On the one hand, spatial multiplexing requires a considerable processing load that depends on the size of the system: number of transmit antennas, number of receivers and bandwidth or number of subcarriers. On the other hand, MIMO techniques require an accurate CSIT. In FDD systems, CSI has to be estimated at the receiver and provided to the transmitter through the feedback channel, hence reducing the efficiency of the system.

This thesis focuses mainly on improving the efficiency of precoding implementations and proposes efficient feedback schemes in MU-MISO systems. This chapter collects and summarizes the conclusions of the various chapters. In addition, possible extensions to the performed research are also discussed. Finally, the last section presents a list of the publications used as reference for this thesis. 


\subsection{Summary}

The thesis is divided in two major parts. The first part contains the chapters related to precoding and the second part contains the chapters related to limited feedback schemes. This section also summarizes the conclusions of the different chapters grouped in two parts.

\subsubsection{Precoding}

Chapter 3 described different precoding algorithms and presented a comparison among them in terms of BER, sum-rate and computational complexity. Among the different precoding algorithms evaluated, those that made use of LR achieved the best BER and sum-rate performance. Regarding the computational complexity, the analysis showed that LR considerably increases the computational complexity of the precoding algorithms. Taking into account performance and computational complexity, the LR-THP algorithm seemed to be the best choice among the LRbased techniques. Hovever, in cases of tighter computational requirements, either ZF or THP should be employed instead. Whereas THP offers a better performance, $\mathrm{ZF}$ can be useful in some systems where linearity is important.

Chapter 4 presented the performance of the most interesting precoding techniques covered in chapter 3 when only imperfect CSIT is available. The analysis showed that two different regions can be identified based on the transmit power: a noiselimited region and an interference-limited region. In the noise-limited region, the performance is limited by the power of the noise at the receiver and the channel errors are almost negligible. Thus, results in this region are similar to the perfect CSIT case. However, in the interference-limited region, the channel errors entail a strong interference that limits the performance of the precoding algorithms. In both regions, LR-THP was the best technique in terms of performance, showing that LR is also a key technique when only imperfect CSIT is available.

Chapter 5 provided details on the implementation of the precoding algorithms on a GPU for a MU-MISO-OFDM system. The GPU implementation was compared with a CPU counterpart, showing that the GPU was able to considerably accelerate the computation by simultaneously processing the calculations associated with the different subcarriers. Results showed that ZF, THP and LR-THP algorithms were more suitable for GPU implementation than other LR-aided precoding techniques as LRAP-lin and LRAP-VB. For this reason, a reconfigurable GPU-based implementation of the THP scheme combined with an LR stage was presented. This implementation allows gating the LR stage off when the user requirements are sufficiently guaranteed by the THP scheme, trading computational cost and performance. The LR stage showed to be the main bottleneck in this parallel implementation. 
In order to improve the LR stage in the implementation of precoding algorithms, several strategies for the parallel implementations of the LLL algorithm were proposed. The CRAS-LLL algorithm allowed carrying out different operations of the LLL algorithm simultaneously in parallel. In addition, the MB-LLL and CRMBLLL algorithms divided the original matrix into several blocks, allowing to perform the CRAS-LLL algorithm in parallel on the different blocks. A heterogeneous platform based on a CPU and a GPU was proposed for the implementation of these algorithms and its performance was compared with implementations running on a GPU with DP capability and a multi-core architecture. Results showed that the use of the GPU allows a significant speed-up compared to multi-core CPUs. In addition, the block concept used in the CRMB-LLL allowed reducing the computational time for large matrices. Regarding the different architectures, the heterogeneous platform achieved the best performance in terms of computational time.

\subsubsection{Limited feedback}

Chapter 6 presented two feedback strategies based on scalar quantization and VQ to exploit the frequency correlation in MU-MISO-OFDM systems. The first strategy consisted of grouping the estimations of the pilot subcarriers into vectors and quantizing them using VQ. The performance achieved with different quantizer configurations was evaluated. Results showed that a higher feedback efficiency was obtained using longer vectors, although its computational complexity increased with the length of those vectors, showing an interesting trade-off between performance and computational complexity. In addition, the different precoding techniques were evaluated under imperfect CSIT achieved with the VQ feedback scheme, validating the results obtained in Chapter 4. The second feedback strategy consisted of using the KL transform to decorrelate the CFR before quantizing it [257]. The proposed scheme was compared with time-domain channel quantization, showing that it achieved a superior performance in systems with unmodulated subcarriers at the edges of the used spectrum, at the expense of a slightly higher computational complexity.

Chapter 7 presented the spatial statistical characterization of the SCM and the Kronecker correlation model. Based on these characterizations, three different channel quantization schemes were presented: DQ, CQ and ACQ. The DQ scheme, based on the characterization of the SCM, showed to outperform the standard quantization scheme in highly correlated environments. However, due to the large number of parameters and the complexity of the SCM, the characterization for a lower correlated environment could not be obtained. In order to obtain a complete characterization for high and low correlated MISO channels, a simpler model such as the Kronecker correlation model was then considered. Based on this characterization, the CQ and ACQ schemes were presented and evaluated using the SCM. Results showed that the proposed schemes outperform other well-known schemes for highly and moderately correlated scenarios. 


\subsection{Future work}

The main lines of research that remain open after this thesis are:

\section{Parallel computation of precoding}

Chapter 5 has presented some parallel implementations of precoding algorithms. Apart from the multicarrier parallelism, the parallelization of the LR stage has been presented. However, the precoding algorithms themselves have not been parallelized due to their sequential nature. This fact is not critical in small systems. However, as the system size increases as in massive MIMO systems, the time required for the precoding increases considerably. Preliminary simulations were performed in massive MIMO systems with a large number of users. To perform the parallelization of the precoding in large MIMO systems, the idea is to group the users into several groups using a clustering algorithm and cancel the interference among the different groups with a linear technique similar to the BD algorithm [47]. Then, the interference among the users within the same group would be cancelled through non-linear precoding since the users would exhibit high correlated channels. Precoding in the different groups would be performed in parallel.

\section{Efficient work distribution on CPU+GPU heterogeneous platforms}

A heterogeneous platform has been proposed in chapter 5 for the implementation of the MB-LLL and CRMB-LLL algorithms. It is important to note that the CPU threads are only responsible for launching the CUDA kernels, not for performing any processing operation. Trying to involve the CPU in the processing, as shown in [235], and finding an efficient way of transferring the data between the CPU and GPU could further reduce the computational time of these algorithms.

\section{Use of VQ for quantizing complex scalar random variables}

Many quantizers such as those in section 6.3 deal with circularly-symmetric complex Gaussian random variables. An extended approach for quantizing these variables would consist of quantizing the real and imaginary parts separately, using the same number of bits for each part and considering the work in [261]. However, a better performance could be obtained by using VQ with vectors of 2 real elements: the real part and the imaginary part of the random variable (see Fig. 6.3b). In addition, a more flexible bit allocation could be achieved since even an odd number of bits would not require a different strategy. Further research could obtain an efficient way for generating the codebooks analytically (avoiding the use of iterative algorithms with training data).

\section{CQ and ACQ based on non-uniform quantization}

The CQ and ACQ schemes presented in chapter 7 are based on uniform quantization. The extension to non-uniform quantization following the work 
in [288] lead to a very complex analysis due to the kind of PDFs involved. Therefore, an adaptation of the Lloyd algorithm presented in chapter 6 to this case would be of interest. In this case, two different codebooks would be updated in each iteration: one for the envelope and the other one for the phase.

\section{Detailed analysis of the performance of ACQ}

The ACQ scheme presented in chapter 7 has demonstrated to outperform the $\mathrm{CQ}$ scheme for the entire range of spatial correlation, and becomes a good option for the CSI feedback in highly and moderately correlated channels. However, a detailed analysis of how the quantization error propagates through every quantization could reveal a better bit allocation and reduce the overall distortion.

\subsection{Publications}

\section{Refereed ISI Journals}

- F. Domene, G. Piñero, M. de Diego, A. Gonzalez, "Channel Quantization Based on the Statistical Characterization of Spatially-Correlated Fading", IEEE Trans. Veh. Technol., to be published. doi:10.1109/TVT.2014.2363170.

- C. M. Józsa, F. Domene, A. M. Vidal, G. Piñero, A. Gonzalez, "High Performance Lattice Reduction on Heterogeneous Computing Platform," The Journal of Supercomputing, Springer US, 1-14, 2014. doi:10.1007/s11227-014-1201-2.

- F. Domene, G. Piñero, C. Botella, A. Gonzalez, "A limited feedback scheme based on spatially correlated channels for coordinated multipoint systems", EURASIP Journal on Wireless Communications and Networking 2012, 176. doi:10.1186/1687-1499-2012-176.

\section{International conferences}

- C. M. Józsa, F. Domene, G. Piñero, A. Gonzalez, A. M. Vidal, "Efficient GPU implementation of Lattice-Reduction-Aided Multiuser Precoding," International Symposium on Wireless Communication Systems (ISWCS), Ilmenau, Germany, August 2013.

- F. Domene, C. M. Józsa, A. M. Vidal, G. Piñero, A. Gonzalez, "Performance analysis of a parallel lattice reduction algorithm on many-core architectures," International Conference on Computational and Mathematical Methods in Science and Engineering (CMMSE), Almería, Spain, June 2013. 
- F. Domene, G. Piñero, "A channel quantization scheme based on the Karhunen-Loève transform," IEEE 7rth Vehicular Technology Conference (VTC 2013-Spring), Dresden, Germany, June 2013. doi:10.1109/VTCSpring.2013.6691816

- F. Domene, S. Roger, C. Ramiro, G. Piñero, A. Gonzalez, "A Reconfigurable GPU Implementation for Tomlinson-Harashima Precoding," IEEE 37th International Conference on Acoustics, Speech, and Signal Processing (ICASSP), Kyoto, Japan, March 2012. doi:10.1109/ICASSP.2012.6288207

- F. Domene, G. Piñero, M. de Diego, A. González, "Performance of Multiuser MIMO-OFDM Precoding Techniques with Quantized Channel Information," International Symposium on Wireless Communication Systems (ISWCS), York, United Kingdom, September 2010. doi:10.1109/ISWCS.2010.5624376

- S. Roger, F. Domene, A. González, V. Almenar and G. Piñero, "An Evaluation of Precoding Techniques for Multiuser Communication Systems," International Symposium on Wireless Communication Systems (ISWCS), York, United Kingdom, September 2010.

doi:10.1109/ISWCS.2010.5624548

\section{National conferences}

- F. Domene, S. Roger, C. Ramiro, G. Piñero, A. González, "Efficient implementation of multiuser precoding algorithms on GPU for MIMO-OFDM systems," Symposium Nacional de la Unión Científica Internacional de Radio (URSI), Elche, Spain, September 2012.

\section{Peer-reviewed non-ISI Journals}

- S. Roger, F. Domene, C. Botella, G. Piñero, A. Gonzalez, V. Almenar, "Recent Advances in MIMO Wireless Systems", Waves, vol. 1, pp. 115-123, 2009. 


\section{Appendices}



Appendix A

CRAS-LLL: CUDA and OpenMP pseudocode 


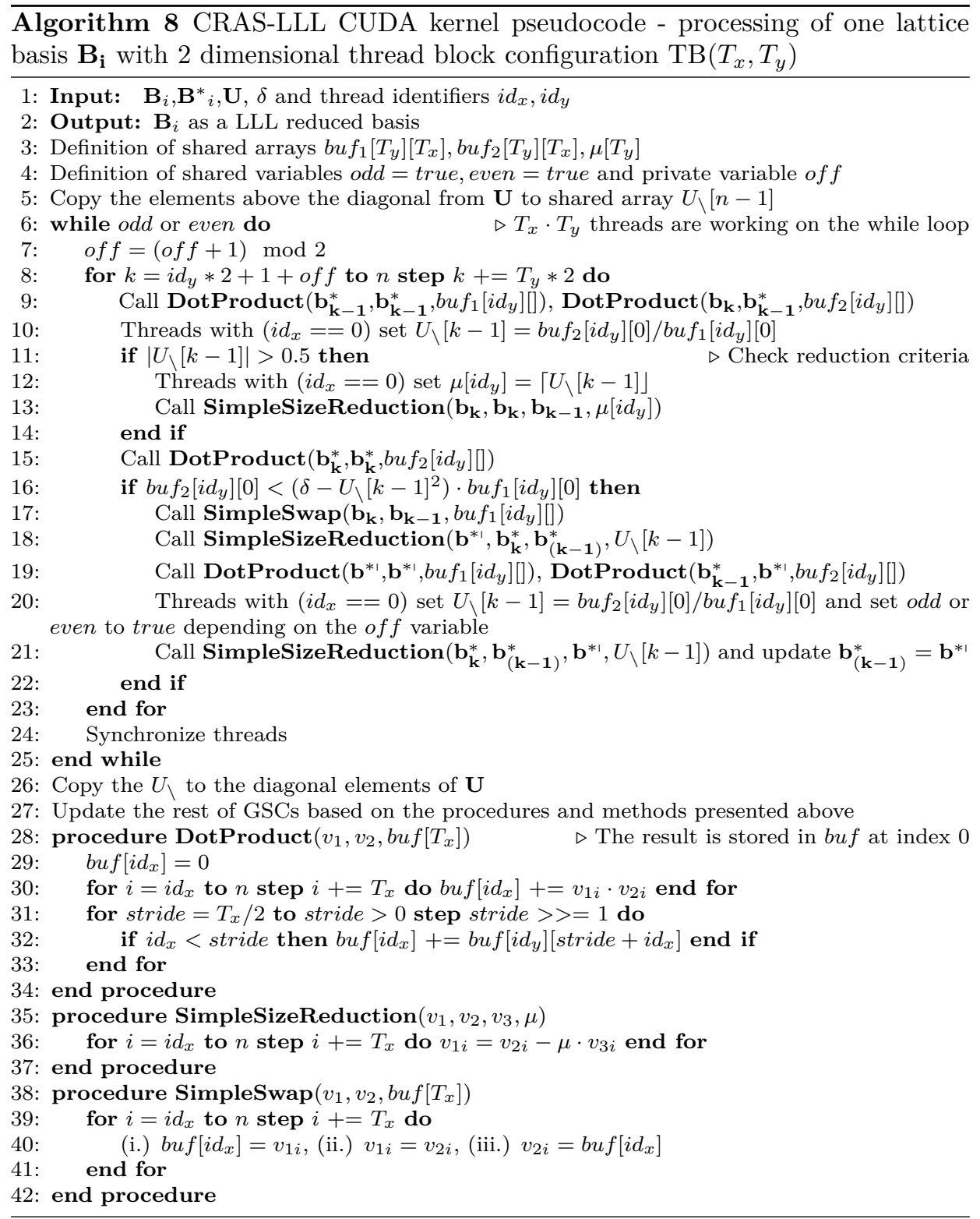




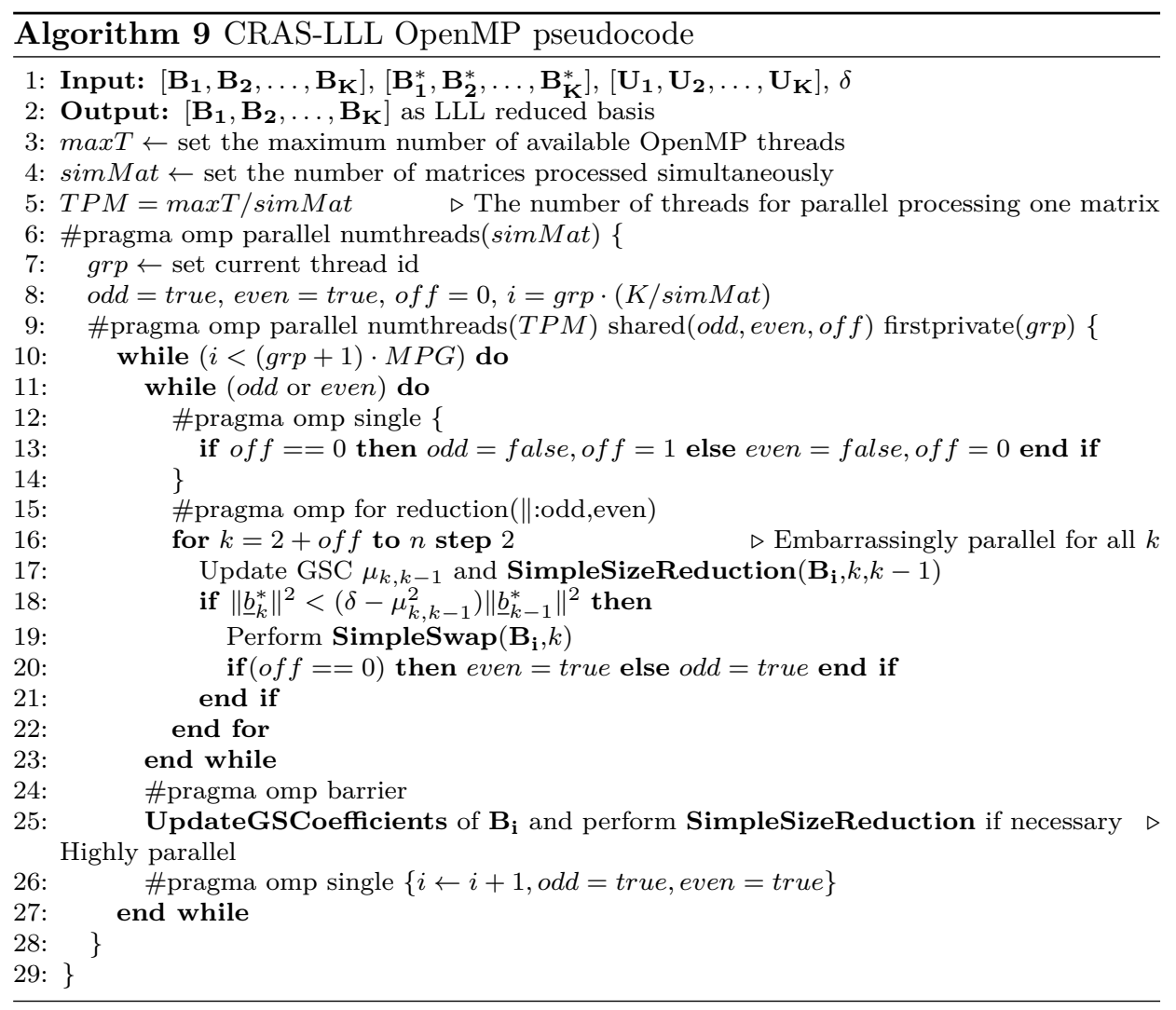





\section{Appendix B}

\section{Conditional quantization codebook: details of calculations}

The distortion in non-reference fading coefficients that is seen in (7.43) can be calculated by making use of the following results. For the conditional probability distribution $f\left(r_{1} \mid r_{2}\right), m_{n}^{a, b}$ is defined as

$$
\begin{aligned}
& m_{n}^{a, b}\left(r_{\mathrm{r}}, \rho\right)=\int_{a}^{b} r_{\mathrm{nr}}^{n} f\left(r_{\mathrm{nr}} \mid r_{\mathrm{r}}\right) \mathrm{d} r_{\mathrm{nr}}= \\
& =\int_{a}^{b} \frac{r_{\mathrm{nr}}^{n+1}}{b_{\mathrm{R}}^{2}\left(1-|\rho|^{2}\right)} \exp \left(-\frac{r_{\mathrm{nr}}^{2}+r_{\mathrm{r}}^{2}|\rho|^{2}}{2 b_{\mathrm{R}}^{2}\left(1-|\rho|^{2}\right)}\right) I_{0}\left(\frac{r_{\mathrm{nr}} r_{\mathrm{r}}|\rho|}{b_{\mathrm{R}}^{2}\left(1-|\rho|^{2}\right)}\right) \mathrm{d} r_{\mathrm{nr}} \\
& =\int_{a}^{b} \frac{r_{\mathrm{nr}}^{n+1}}{b_{\mathrm{R}}^{2}\left(1-|\rho|^{2}\right)} \exp \left(-\frac{r_{\mathrm{nr}}^{2}+r_{\mathrm{r}}^{2}|\rho|^{2}}{2 b_{\mathrm{R}}^{2}\left(1-|\rho|^{2}\right)}\right) \sum_{k=0}^{\infty} \frac{\left(r_{\mathrm{nr}} r_{\mathrm{r}}|\rho|\right)^{2 k}}{\left(2 b_{\mathrm{R}}^{2}\left(1-|\rho|^{2}\right)\right)^{2 k}(k !)^{2}} \mathrm{~d} r_{\mathrm{nr}},
\end{aligned}
$$

where the Taylor series expansion around $x=0$ of the modified Bessel function of the first kind with order 0 has been used [286, Sec. 9],

$$
I_{0}(x)=\sum_{k=0}^{\infty} \frac{x^{2 k}}{2^{2 k}(k !)^{2}} .
$$

Defining $A=2 b_{\mathrm{R}}^{2}\left(1-|\rho|^{2}\right), C=r_{\mathrm{r}}^{2}|\rho|^{2}$ and using a change of variables $t=r_{\mathrm{nr}}^{2} / A$, $m_{n}^{a, b}\left(r_{\mathrm{r}}, \rho\right)$ can be expressed as

$$
\begin{aligned}
& m_{n}^{a, b}\left(r_{\mathrm{r}}, \rho\right)= \\
& \quad \exp \left(\frac{-C}{A}\right) \sum_{k=0}^{\infty} \frac{C^{k}\left(\Gamma\left(k+\frac{n}{2}+1, \frac{a^{2}}{A}\right)-\Gamma\left(k+\frac{n}{2}+1, \frac{b^{2}}{A}\right)\right)}{A^{k-\frac{n}{2}}(k !)^{2}},
\end{aligned}
$$


where $\Gamma(s, x)$ denotes the upper incomplete Gamma function [286, Sec. 6]. When $a=0$ and $b \rightarrow \infty, m_{n}^{a, b}\left(r_{\mathrm{r}}, \rho\right)$ becomes the moment taken about 0 , also known as raw moment, which has been expressed in (7.27).

Equation (B.3) may lead to overflow/underflow problems, since the numerator and denominator contain very large values such as the Gamma function or the squared factorial. In order to avoid it, $Q(s, x)$ can be defined as

$$
Q(s, x)=\frac{1}{\Gamma(s)} \int_{x}^{\infty} e^{-t} t^{s-1} \mathrm{~d} t
$$

which is a bounded function between 0 and 1 . MATLAB provides the gammainc function which computes $Q(s, x)$ avoiding overflow. Thus, the upper incomplete Gamma function can be expressed as $\Gamma(s, x)=\Gamma(s) Q(s, x)$, and (B.3) turns into

$$
\begin{aligned}
m_{n}^{a, b}\left(r_{\mathrm{r}}, \rho\right)= & \exp \left(\frac{-C}{A}\right) \times \\
& \sum_{k=0}^{\infty} \frac{C^{k} \Gamma\left(k+\frac{n}{2}+1\right)}{A^{k-\frac{n}{2}}(k !)^{2}}\left(\Gamma\left(k+\frac{n}{2}+1, \frac{a^{2}}{A}\right)-\Gamma\left(k+\frac{n}{2}+1, \frac{b^{2}}{A}\right)\right),
\end{aligned}
$$

Therefore, the overflow/underflow problem is now located in the factor

$$
d_{k}=\frac{C^{k} \Gamma\left(k+\frac{n}{2}+1\right)}{A^{k-\frac{n}{2}}(k !)^{2}} .
$$

Since the moments of interest are the 0th, 1st and 2nd, let us focus on these particular cases.

- $n=0$

Considering that $\Gamma(s)=(s-1)$ !, (B.6) results into

$$
d_{k}=\frac{C^{k}}{A^{k} k !} \text {. }
$$

It is interesting to realize that, in order to calculate the $d_{k}$ coefficients in an efficient way, they can be expressed recursively as

$$
\begin{aligned}
& d_{0}=1, \\
& d_{k}=\frac{C}{k A} d_{k-1}, \quad k=1,2,3 \ldots
\end{aligned}
$$

$n=1$

Considering that

$$
\Gamma\left(s+\frac{1}{2}\right)=\frac{(2 s) !}{4^{s} s !} \sqrt{\pi}
$$


(B.6) results into

$$
\begin{aligned}
d_{k} & =\sqrt{A}\left(\frac{C}{A}\right)^{k} \frac{(2 k+2) ! \sqrt{\pi}}{4^{k+1}(k !)^{3}(k+1)} \\
& =\sqrt{A}\left(\frac{C}{A}\right)^{k} \frac{\sqrt{\pi} \prod_{k^{\prime}=0}^{k}\left(k+2+k^{\prime}\right)}{4^{k+1}(k !)^{2}} .
\end{aligned}
$$

Manipulating the equation, the coefficients $d_{k}$ can be expressed recursively as

$$
\begin{aligned}
& d_{0}=\frac{\sqrt{\pi A}}{2}, \\
& d_{k}=\frac{(2 k+1) C}{2 k^{2} A} d_{k-1}, \quad k=1,2,3 \ldots
\end{aligned}
$$

$n=2$

Considering that $\Gamma(s)=(s-1)$ !, (B.6) results into

$$
d_{k}=A\left(\frac{C}{A}\right)^{k} \frac{k+1}{k !} .
$$

In this case, the coefficients $d_{k}$ can be expressed recursively as

$$
\begin{aligned}
& d_{0}=A \\
& d_{k}=\frac{(k+1) C}{k^{2} A} d_{k-1}, \quad k=1,2,3 \ldots
\end{aligned}
$$

The following result, which is related to the phase deviation $\Delta$, also appears as a part of (7.43) and, assuming that $-\pi \leq \Delta_{p-1} \leq \Delta_{p}<\pi$, the result can be obtained through an integration by parts:

$$
\begin{aligned}
& \int_{\Delta_{p-1}}^{\Delta_{p}} \cos \left(\Delta-\hat{\Delta}_{p}\right) \tilde{f}_{\Delta}(\Delta, \rho) \mathrm{d} \Delta= \\
& \int_{\Delta_{p-1}}^{\Delta_{p}} \cos \left(\Delta-\hat{\Delta}_{p}\right) \frac{\exp \left(-|\Delta| / b_{\mathrm{L}, n}\right)}{2 b_{\mathrm{L}, n}\left(1-\exp \left(-\pi / b_{\mathrm{L}, n}\right)\right)} \mathrm{d} \Delta= \\
& \quad \frac{1}{2\left(1-\exp \left(-\pi / b_{\mathrm{L}, n}\right)\right)\left(1+b_{\mathrm{L}, n}^{2}\right)}\left[\left(\operatorname{sgn}\left(\Delta_{p}\right)-\operatorname{sgn}\left(\Delta_{p-1}\right)\right) \cos \left(\hat{\Delta}_{p}\right)\right. \\
& \quad-\exp \left(\frac{-\left|\Delta_{p-1}\right|}{b_{\mathrm{L}, n}}\right)\left(b \sin \left(\Delta_{p-1}-\hat{\Delta}_{p}\right)-\operatorname{sgn}\left(\Delta_{p-1}\right) \cos \left(\Delta_{p-1}-\hat{\Delta}_{p}\right)\right) \\
& \left.\quad+\exp \left(\frac{-\left|\Delta_{p}\right|}{b_{\mathrm{L}, n}}\right)\left(b \sin \left(\Delta_{p}-\hat{\Delta}_{p}\right)-\operatorname{sgn}\left(\Delta_{p}\right) \cos \left(\Delta_{p}-\hat{\Delta}_{p}\right)\right)\right],
\end{aligned}
$$

where $b_{\mathrm{L}, n}$ is given by $(7.45)$. 



\section{Bibliography}

[1] Ericsson, "Ericsson mobility report, on the pulse of the networked society," June 2014. [Online]. Available: http://www.ericsson.com/res/docs/2014/ ericsson-mobility-report-june-2014.pdf

[2] C. V. N. I. Cisco, "Global mobile data traffic forecast update, 2013-2018," Cisco Visual Networking Index (VNI) Forecast, 2013.

[3] B. Sanou, "The world in 2014: Ict facts and figures," International Telecommunications Union, 2014. [Online]. Available: http://www.itu.int/ en/ITU-D/Statistics/Documents/facts/ICTFactsFigures2014-e.pdf

[4] D. Gesbert, M. Shafi, D. shan Shiu, P. J. Smith, and A. Naguib, "From theory to practice: an overview of MIMO space-time coded wireless systems," IEEE J. Sel. Areas Commun., vol. 21, no. 3, pp. 281-302, 2003. [Online]. Available: http://ieeexplore.ieee.org/stamp/stamp.jsp?arnumber= 1192168

[5] A. J. Paulraj, D. A. Gore, R. U. Nabar, and H. Bolcskei, "An overview of MIMO communications - a key to gigabit wireless," Proceedings of the IEEE, vol. 92, no. 2, pp. 198-218, 2004. [Online]. Available: http://ieeexplore.ieee.org/stamp/stamp.jsp?arnumber $=1266912$

[6] D. Gesbert, M. Kountouris, R. W. Heath, C.-B. Chae, and T. Salzer, "Shifting the MIMO paradigm," IEEE Signal Process. Mag., vol. 24, no. 5, pp. 36-46, 2007. [Online]. Available: http://ieeexplore.ieee.org/stamp/ stamp.jsp?arnumber $=4350224$

[7] G. L. Stuber, J. R. Barry, S. W. McLaughlin, Y. Li, M. A. Ingram, and T. G. Pratt, "Broadband MIMO-OFDM wireless communications," Proc. IEEE, vol. 92, no. 2, pp. 271-294, 2004. [Online]. Available: http://ieeexplore.ieee.org/stamp/stamp.jsp?arnumber $=1266914$

[8] M. Jiang and L. Hanzo, "Multiuser MIMO-OFDM for next-generation wireless systems," Proc. IEEE, vol. 95, no. 7, pp. 1430-1469, 2007. [Online]. Available: http://ieeexplore.iee.org/stamp/stamp.jsp?arnumber $=4287201$ 
[9] E. Perahia, "IEEE 802.11n development: History, process, and technology," IEEE Commun. Mag., vol. 46, no. 7, pp. 48-55, 2008. [Online]. Available: http://ieeexplore.ieee.org/stamp/stamp.jsp?arnumber $=4557042$

[10] IEEE 802.16 Working Group, "IEEE standard for local and metropolitan area networks. Part 16: Air interface for fixed and mobile broadband wireless access systems." IEEE Standard, vol. 802, 2006.

[11] S. Sesia, I. Toufik, and M. Baker, LTE, The UMTS Long Term Evolution: From Theory to Practice, 2nd ed. Wiley, 2011.

[12] J. Andrews, S. Buzzi, W. Choi, S. Hanly, A. Lozano, A. Soong, and J. Zhang, "What will $5 \mathrm{~g}$ be?" IEEE J. Sel. Areas Commun., vol. 32, no. 6, pp. 1065-1082, 2014. [Online]. Available: http: //ieeexplore.ieee.org/stamp/stamp.jsp?arnumber $=6824752$

[13] D. J. Love, R. W. Heath, V. K. N. Lau, D. Gesbert, B. D. Rao, and M. Andrews, "An overview of limited feedback in wireless communication systems," IEEE J. Sel. Areas Commun., vol. 26, no. 8, pp. 1341-1365, 2008. [Online]. Available: http://ieeexplore.ieee.org/stamp/stamp.jsp?arnumber= 4641946

[14] A. K. Lenstra, H. W. Lenstra, and L. Lovász, "Factoring polynomials with rational coefficients," Mathematische Annalen, vol. 261, no. 4, pp. 515-534, 1982. [Online]. Available: http://www.springerlink.com/index/ $\operatorname{lh} 1 \mathrm{~m} 24436431 \mathrm{~g} 068 . \mathrm{pdf}$

[15] C. Windpassinger, R. F. H. Fischer, and J. B. Huber, "Lattice-reductionaided broadcast precoding," IEEE Trans. Commun., vol. 52, no. 12, pp. 2057-2060, 2004. [Online]. Available: http://ieeexplore.ieee.org/stamp/ stamp.jsp?arnumber $=1369614$

[16] D. Xu, Y. Huang, and L. Yang, "Improved nonlinear multiuser precoding using lattice reduction," Signal, image and video processing, vol. 3, no. 1, pp. 47-52, 2009. [Online]. Available: http://www.springerlink.com/index/ 6R4568460017Q027.pdf

[17] M. Wu, Y. Sun, S. Gupta, and J. R. Cavallaro, "Implementation of a high throughput soft mimo detector on gpu," Journal of Signal Processing Systems, vol. 64, no. 1, pp. 123-136, 2011. [Online]. Available: http://www.springerlink.com/index/T604N2217G1N83LU.pdf

[18] M. Garland, S. Le Grand, J. Nickolls, J. Anderson, J. Hardwick, S. Morton, E. Phillips, Y. Zhang, and V. Volkov, "Parallel computing experiences with CUDA," IEEE Micro, vol. 28, no. 4, pp. 13-27, 2008. [Online]. Available: http://ieeexplore.ieee.org/stamp/stamp.jsp?arnumber $=4626815$ 
[19] J. Nickolls and W. Dally, "The GPU computing era," IEEE Micro, vol. 30, no. 2, pp. 56-69, 2010. [Online]. Available: http://ieeexplore.ieee.org/ stamp/stamp.jsp?arnumber $=5446251$

[20] C. B. Peel, B. M. Hochwald, and A. L. Swindlehurst, "A vector-perturbation technique for near-capacity multiantenna multiuser communication - Part I: channel inversion and regularization," IEEE Trans. Commun., vol. 53, no. 1, pp. 195-202, 2005. [Online]. Available: http://ieeexplore.ieee.org/ stamp/stamp.jsp?arnumber $=1391204$

[21] C. Windpassinger, R. F. H. Fischer, T. Vencel, and J. B. Huber, "Precoding in multiantenna and multiuser communications," IEEE Trans. Wireless Commun., vol. 3, no. 4, pp. 1305-1316, 2004. [Online]. Available: http://ieeexplore.ieee.org/stamp/stamp.jsp?arnumber $=1310319$

[22] B. M. Hochwald, C. B. Peel, and A. L. Swindlehurst, "A vector-perturbation technique for near-capacity multiantenna multiuser communication-part II: perturbation," IEEE Trans. Commun., vol. 53, no. 3, pp. 537-544, 2005. [Online]. Available: http://ieeexplore.ieee.org/stamp/stamp.jsp?arnumber $=$ 1413598

[23] G. J. Foschini and M. J. Gans, "On limits of wireless communications in a fading environment when using multiple antennas," Wireless personal communications, vol. 6, no. 3, pp. 311-335, 1998. [Online]. Available: http://link.springer.com/article/10.1023/A\%3A1008889222784

[24] E. Telatar, "Capacity of multi-antenna gaussian channels," European transactions on telecommunications, vol. 10, no. 6, pp. 585-595, 1999. [Online]. Available: http://onlinelibrary.wiley.com/doi/10.1002/ett. 4460100604/full

[25] G. Raleigh and J. Cioffi, "Spatio-temporal coding for wireless communication," IEEE Trans. Commun., vol. 46, no. 3, pp. 357-366, 1998. [Online]. Available: http://ieeexplore.ieee.org/stamp/stamp.jsp?arnumber $=662641$

[26] A. Goldsmith, S. A. Jafar, N. Jindal, and S. Vishwanath, "Capacity limits of MIMO channels," IEEE J. Sel. Areas Commun., vol. 21, no. 5, pp. 684-702, 2003. [Online]. Available: http://ieeexplore.ieee.org/stamp/stamp. jsp?arnumber $=1203154$

[27] E. Biglieri, R. Calderbank, A. Constantinides, A. Goldsmith, A. Paulraj, and H. V. Poor, MIMO Wireless Communications. Cambridge University Press, 2007. [Online]. Available: http://dx.doi.org/10.1017/CBO9780511618420

[28] T. M. Cover and J. A. Thomas, Elements of Information Theory. WileyInterscience, 2006. 
[29] V. Tarokh, N. Seshadri, and A. Calderbank, "Space-time codes for high data rate wireless communication: performance criterion and code construction," IEEE Trans. Inf. Theory, vol. 44, no. 2, pp. 744-765, 1998. [Online]. Available: http://ieeexplore.ieee.org/stamp/stamp.jsp?arnumber $=661517$

[30] S. Alamouti, "A simple transmit diversity technique for wireless communications," IEEE J. Sel. Areas Commun., vol. 16, no. 8, pp. 1451-1458, 1998. [Online]. Available: http://ieeexplore.ieee.org/stamp/ stamp.jsp?arnumber $=730453$

[31] G. J. Foschini, "Layered space-time architecture for wireless communication in a fading environment when using multi-element antennas," Bell labs technical journal, vol. 1, no. 2, pp. 41-59, 1996. [Online]. Available: http://onlinelibrary.wiley.com/doi/10.1002/bltj.2015/full

[32] E. Larsson, "MIMO detection methods: How they work [lecture notes]," IEEE Signal Process. Mag., vol. 26, no. 3, pp. 91-95, 2009. [Online]. Available: http://ieeexplore.ieee.org/stamp/stamp.jsp?arnumber $=4815548$

[33] J. R. Barry, E. A. Lee, and D. G. Messerschmitt, Digital Communication, 3rd ed. Springer, 2004.

[34] L. Zheng and D. Tse, "Diversity and multiplexing: a fundamental tradeoff in multiple-antenna channels," IEEE Trans. Inf. Theory, vol. 49, no. 5, pp. 1073-1096, 2003. [Online]. Available: http: //ieeexplore.ieee.org/stamp/stamp.jsp?arnumber $=1197843$

[35] D. Tse and P. Viswanath, Fundamentals of wireless communication. New York, NY, USA: Cambridge University Press, 2005.

[36] H. Bölcskei, D. Gesbert, C. Papadias, A. J. Van Der Veen, and A. M. Cipriano, Space-Time Wireless Systems: From Array Processing to MIMO Communications. Cambridge Univ, 2006.

[37] W. Yu, W. Rhee, S. Boyd, and J. Cioffi, "Iterative water-filling for Gaussian vector multiple-access channels," IEEE Trans. Inf. Theory, vol. 50, no. 1, pp. 145-152, 2004. [Online]. Available: http://ieeexplore.ieee.org/stamp/ stamp.jsp?arnumber $=1262622$

[38] W. Yu and T. Lan, "Transmitter optimization for the multi-antenna downlink with per-antenna power constraints," IEEE Trans. Signal Process., vol. 55, no. 6, pp. 2646-2660, 2007. [Online]. Available: http://ieeexplore.ieee.org/stamp/stamp.jsp?arnumber $=4203115$

[39] Q. Spencer, C. Peel, A. Swindlehurst, and M. Haardt, "An introduction to the multi-user MIMO downlink," IEEE Commun. Mag., vol. 42, no. 10, pp. 60-67, 2004. [Online]. Available: http://ieeexplore.ieee.org/stamp/stamp. jsp?arnumber $=1341262$ 
[40] M. Costa, "Writing on dirty paper (corresp.)," IEEE Trans. Inf. Theory, vol. 29, no. 3, pp. 439-441, 1983. [Online]. Available: http://ieeexplore.ieee.org/stamp/stamp.jsp?arnumber $=1056659$

[41] G. Caire and S. Shamai, "On the achievable throughput of a multiantenna Gaussian broadcast channel," IEEE Trans. Inf. Theory, vol. 49, no. 7, pp. 1691-1706, 2003. [Online]. Available: http: //ieeexplore.ieee.org/stamp/stamp.jsp?arnumber $=1207369$

[42] P. Viswanath and D. Tse, "Sum capacity of the vector Gaussian broadcast channel and uplink-downlink duality," IEEE Trans. Inf. Theory, vol. 49, no. 8, pp. 1912-1921, 2003. [Online]. Available: http://ieeexplore.ieee.org/stamp/stamp.jsp?arnumber $=1214071$

[43] W. Yu and J. Cioffi, "Sum capacity of Gaussian vector broadcast channels," IEEE Trans. Inf. Theory, vol. 50, no. 9, pp. 1875-1892, 2004. [Online]. Available: http://ieeexplore.ieee.org/stamp/stamp.jsp?arnumber $=1327794$

[44] H. Weingarten, Y. Steinberg, and S. Shamai, "The capacity region of the Gaussian multiple-input multiple-output broadcast channel," IEEE Trans. Inf. Theory, vol. 52, no. 9, pp. 3936-3964, 2006. [Online]. Available: http://ieeexplore.ieee.org/stamp/stamp.jsp?arnumber $=1683918$

[45] S. Vishwanath, N. Jindal, and A. Goldsmith, "Duality, achievable rates, and sum-rate capacity of Gaussian MIMO broadcast channels," IEEE Trans. Inf. Theory, vol. 49, no. 10, pp. 2658-2668, 2003. [Online]. Available: http://ieeexplore.ieee.org/stamp/stamp.jsp?arnumber $=1237143$

[46] N. Jindal, S. Vishwanath, and A. Goldsmith, "On the duality of Gaussian multiple-access and broadcast channels," IEEE Trans. Inf. Theory, vol. 50, no. 5, pp. 768-783, 2004. [Online]. Available: http://ieeexplore.ieee.org/stamp/stamp.jsp?arnumber $=1291726$

[47] Q. H. Spencer, A. L. Swindlehurst, and M. Haardt, "Zero-forcing methods for downlink spatial multiplexing in multiuser MIMO channels," IEEE Trans. Signal Process., vol. 52, no. 2, pp. 461-471, 2004. [Online]. Available: http://ieeexplore.ieee.org/stamp/stamp.jsp?arnumber $=1261332$

[48] P. Gupta and P. Kumar, "The capacity of wireless networks," IEEE Trans. Inf. Theory, vol. 46, no. 2, pp. 388-404, 2000. [Online]. Available: http://ieeexplore.ieee.org/stamp/stamp.jsp?arnumber $=825799$

[49] D. Gesbert, S. Hanly, H. Huang, S. Shamai Shitz, O. Simeone, and W. Yu, "Multi-cell MIMO cooperative networks: A new look at interference," IEEE J. Sel. Areas Commun., vol. 28, no. 9, pp. 1380-1408, 2010. [Online]. Available: http://ieeexplore.ieee.org/stamp/stamp.jsp?arnumber $=5594708$ 
[50] M. H. M. Costa and A. Gamal, "The capacity region of the discrete memoryless interference channel with strong interference (corresp.)," IEEE Trans. Inf. Theory, vol. 33, no. 5, pp. 710-711, 1987. [Online]. Available: http://ieeexplore.ieee.org/stamp/stamp.jsp?arnumber $=1057340$

[51] S. Shamai and A. Wyner, "Information-theoretic considerations for symmetric, cellular, multiple-access fading channels. ii," IEEE Trans. Inf. Theory, vol. 43, no. 6, pp. 1895-1911, 1997. [Online]. Available: http://ieeexplore.ieee.org/stamp/stamp.jsp?arnumber $=641554$

[52] S. Catreux, P. Driessen, and L. Greenstein, "Attainable throughput of an interference-limited multiple-input multiple-output (MIMO) cellular system," IEEE Trans. Commun., vol. 49, no. 8, pp. 1307-1311, 2001. [Online]. Available: http://ieeexplore.ieee.org/stamp/stamp.jsp?arnumber= 939839

[53] A. Lozano and A. Tulino, "Capacity of multiple-transmit multiple-receive antenna architectures," IEEE Trans. Inf. Theory, vol. 48, no. 12, pp. 3117-3128, 2002. [Online]. Available: http://ieeexplore.ieee.org/stamp/ stamp.jsp?arnumber $=1077806$

[54] S. Jafar and M. Fakhereddin, "Degrees of freedom for the MIMO interference channel," IEEE Trans. Inf. Theory, vol. 53, no. 7, pp. 2637-2642, 2007. [Online]. Available: http://ieeexplore.ieee.org/stamp/ stamp.jsp?arnumber $=4252335$

[55] C.-X. Wang, X. Hong, X. Ge, X. Cheng, G. Zhang, and J. Thompson, "Cooperative MIMO channel models: A survey," IEEE Commun. Mag., vol. 48, no. 2, pp. 80-87, 2010. [Online]. Available: http: //ieeexplore.ieee.org/stamp/stamp.jsp?arnumber $=5402668$

[56] S. Venkatesan, A. Lozano, and R. Valenzuela, "Network MIMO: Overcoming intercell interference in indoor wireless systems," in Signals, Systems and Computers, 200\%. ACSSC 200\%. Conference Record of the Forty-First Asilomar Conference on, 2007, pp. 83-87. [Online]. Available: http://ieeexplore.ieee.org/stamp/stamp.jsp?arnumber $=4487170$

[57] H. Zhang and H. Dai, "Cochannel interference mitigation and cooperative processing in downlink multicell multiuser mimo networks," EURASIP Journal on Wireless Communications and Networking, vol. 2004, no. 2, pp. 222-235, 2004. [Online]. Available: http://dl.acm.org/citation.cfm?id= 1086473

[58] M. Karakayali, G. Foschini, and R. Valenzuela, "Network coordination for spectrally efficient communications in cellular systems," IEEE Wireless Commun. Mag., vol. 13, no. 4, pp. 56-61, 2006. [Online]. Available: http://ieeexplore.ieee.org/stamp/stamp.jsp?arnumber $=1678166$ 
[59] G. Foschini, K. Karakayali, and R. Valenzuela, "Coordinating multiple antenna cellular networks to achieve enormous spectral efficiency," IEE Proceedings- Communications, vol. 153, no. 4, pp. 548-555, 2006. [Online]. Available: http://ieeexplore.ieee.org/stamp/stamp.jsp?arnumber $=1664084$

[60] W. Hardjawana, B. Vucetic, and Y. Li, "Multi-user cooperative base station systems with joint precoding and beamforming," IEEE J. Sel. Topics Signal Process., vol. 3, no. 6, pp. 1079-1093, 2009. [Online]. Available: http://ieeexplore.ieee.org/stamp/stamp.jsp?arnumber $=5370662$

[61] R. Zhang, "Cooperative multi-cell block diagonalization with per-basestation power constraints," IEEE J. Sel. Areas Commun., vol. 28, no. 9, pp. 1435-1445, 2010. [Online]. Available: http://ieeexplore.ieee.org/stamp/ stamp.jsp?arnumber $=5594707$

[62] P. Marsch and G. Fettweis, "On base station cooperation schemes for downlink network MIMO under a constrained backhaul," in Global Telecommunications Conference, 2008. IEEE GLOBECOM 2008. IEEE, 2008, pp. 1-6. [Online]. Available: http://ieeexplore.ieee.org/stamp/stamp. jsp?arnumber $=4698016$

[63] E. Björnson, R. Zakhour, D. Gesbert, and B. Ottersten, "Cooperative multicell precoding: Rate region characterization and distributed strategies with instantaneous and statistical CSI," IEEE Trans. Signal Process., vol. 58, no. 8, pp. 4298-4310, 2010. [Online]. Available: http://ieeexplore.ieee.org/stamp/stamp.jsp?arnumber $=5462883$

[64] T. Biermann, L. Scalia, C. Choi, W. Kellerer, and H. Karl, "How backhaul networks influence the feasibility of coordinated multipoint in cellular networks [accepted from open call]," IEEE Commun. Mag., vol. 51, no. 8, pp. 168-176, 2013. [Online]. Available: http: //ieeexplore.ieee.org/stamp/stamp.jsp?arnumber $=6576356$

[65] H. Zhang, N. Mehta, A. Molisch, J. Zhang, and H. Dai, "Asynchronous interference mitigation in cooperative base station systems," IEEE Trans. Wireless Commun., vol. 7, no. 1, pp. 155-165, 2008. [Online]. Available: http://ieeexplore.ieee.org/stamp/stamp.jsp?arnumber $=4432257$

[66] 3GPP TR 36.819 V11.2.0, "Coordinated multi-point operation for LTE physical layer aspects (Release 11)."

[67] M. Sawahashi, Y. Kishiyama, A. Morimoto, D. Nishikawa, and M. Tanno, "Coordinated multipoint transmission/reception techniques for LTE-advanced [coordinated and distributed MIMO]," IEEE Wireless Commun. Mag., vol. 17, no. 3, pp. 26-34, 2010. [Online]. Available: http://ieeexplore.ieee.org/stamp/stamp.jsp?arnumber $=5490976$ 
[68] P. Marsch and G. P. Fettweis, Coordinated Multi-Point in Mobile Communications: from theory to practice. Cambridge University Press, 2011.

[69] J. Lee, Y. Kim, H. Lee, B. L. Ng, D. Mazzarese, J. Liu, W. Xiao, and Y. Zhou, "Coordinated multipoint transmission and reception in LTE-advanced systems," IEEE Commun. Mag., vol. 50, no. 11, pp. 44-50, 2012. [Online]. Available: http://ieeexplore.ieee.org/stamp/stamp. jsp?arnumber $=6353681$

[70] R. Irmer, H. P. Mayer, A. Weber, V. Braun, M. Schmidt, M. Ohm, N. Ahr, A. Zoch, C. Jandura, P. Marsch, and G. Fettweis, "Multisite field trial for LTE and advanced concepts," IEEE Commun. Mag., vol. 47, no. 2, pp. 92-98, 2009. [Online]. Available: http://ieeexplore.ieee.org/stamp/stamp. jsp?arnumber $=4785385$

[71] R. Irmer, H. Droste, P. Marsch, M. Grieger, G. Fettweis, S. Brueck, H.-P. Mayer, L. Thiele, and V. Jungnickel, "Coordinated multipoint: Concepts, performance, and field trial results," IEEE Commun. Mag., vol. 49, no. 2, pp. 102-111, 2011. [Online]. Available: http: //ieeexplore.ieee.org/stamp/stamp.jsp?arnumber $=5706317$

[72] F. Rusek, D. Persson, B. K. Lau, E. Larsson, T. Marzetta, O. Edfors, and F. Tufvesson, "Scaling up MIMO: Opportunities and challenges with very large arrays," IEEE Signal Process. Mag., vol. 30, no. 1, pp. 40-60, 2013. [Online]. Available: http://ieeexplore.ieee.org/stamp/stamp.jsp?arnumber= 6375940

[73] E. Larsson, O. Edfors, F. Tufvesson, and T. Marzetta, "Massive MIMO for next generation wireless systems," IEEE Commun. Mag., vol. 52, no. 2, pp. 186-195, 2014. [Online]. Available: http://ieeexplore.ieee.org/stamp/stamp. jsp?arnumber $=6736761$

[74] T. Marzetta, "Noncooperative cellular wireless with unlimited numbers of base station antennas," IEEE Trans. Wireless Commun., vol. 9, no. 11, pp. 3590-3600, 2010. [Online]. Available: http://ieeexplore.ieee.org/stamp/ stamp.jsp?arnumber $=5595728$

[75] J. Jose, A. Ashikhmin, T. Marzetta, and S. Vishwanath, "Pilot contamination and precoding in multi-cell TDD systems," IEEE Trans. Wireless Commun., vol. 10, no. 8, pp. 2640-2651, 2011. [Online]. Available: http://ieeexplore.ieee.org/stamp/stamp.jsp?arnumber $=5898372$

[76] F. Fernandes, A. Ashikhmin, and T. Marzetta, "Inter-cell interference in noncooperative TDD large scale antenna systems," IEEE J. Sel. Areas Commun., vol. 31, no. 2, pp. 192-201, 2013. [Online]. Available: http://ieeexplore.ieee.org/stamp/stamp.jsp?arnumber $=6415391$ 
[77] H. Yin, D. Gesbert, M. Filippou, and Y. Liu, "A coordinated approach to channel estimation in large-scale multiple-antenna systems," IEEE J. Sel. Areas Commun., vol. 31, no. 2, pp. 264-273, 2013. [Online]. Available: http://ieeexplore.ieee.org/stamp/stamp.jsp?arnumber $=6415397$

[78] L. Dai, Z. Wang, and Z. Yang, "Spectrally efficient time-frequency training OFDM for mobile large-scale MIMO systems," IEEE J. Sel. Areas Commun., vol. 31, no. 2, pp. 251-263, 2013. [Online]. Available: http://ieeexplore.ieee.org/stamp/stamp.jsp?arnumber $=6415396$

[79] H. Ngo, E. Larsson, and T. Marzetta, "The multicell multiuser MIMO uplink with very large antenna arrays and a finite-dimensional channel," IEEE Trans. Commun., vol. 61, no. 6, pp. 2350-2361, 2013. [Online]. Available: http://ieeexplore.ieee.org/stamp/stamp.jsp?arnumber=6493983

[80] Y.-H. Nam, B. L. Ng, K. Sayana, Y. Li, J. Zhang, Y. Kim, and J. Lee, "Full-dimension MIMO (fd-MIMO) for next generation cellular technology," IEEE Commun. Mag., vol. 51, no. 6, pp. 172-179, 2013. [Online]. Available: http://ieeexplore.ieee.org/stamp/stamp.jsp?arnumber $=6525612$

[81] C. Masouros, M. Sellathurai, and T. Ratnarajah, "Large-scale MIMO transmitters in fixed physical spaces: The effect of transmit correlation and mutual coupling," IEEE Trans. Commun., vol. 61, no. 7, pp. 2794-2804, 2013. [Online]. Available: http://ieeexplore.ieee.org/stamp/ stamp.jsp?arnumber $=6522419$

[82] H. Huh, G. Caire, H. Papadopoulos, and S. Ramprashad, "Achieving "massive MIMO" spectral efficiency with a not-so-large number of antennas," IEEE Trans. Wireless Commun., vol. 11, no. 9, pp. 3226-3239, 2012. [Online]. Available: http://ieeexplore.ieee.org/stamp/stamp.jsp?arnumber= 6241389

[83] J. Hoydis, S. ten Brink, and M. Debbah, "Massive MIMO in the UL/DL of cellular networks: How many antennas do we need?" IEEE J. Sel. Areas Commun., vol. 31, no. 2, pp. 160-171, 2013. [Online]. Available: http://ieeexplore.ieee.org/stamp/stamp.jsp?arnumber $=6415388$

[84] H. Yang and T. Marzetta, "Performance of conjugate and zero-forcing beamforming in large-scale antenna systems," IEEE J. Sel. Areas Commun., vol. 31, no. 2, pp. 172-179, 2013. [Online]. Available: http://ieeexplore.ieee.org/stamp/stamp.jsp?arnumber $=6415389$

[85] W. Yang, G. Durisi, and E. Riegler, "On the capacity of large-MIMO block-fading channels," IEEE J. Sel. Areas Commun., vol. 31, no. 2, pp. 117-132, 2013. [Online]. Available: http://ieeexplore.ieee.org/stamp/stamp. jsp?arnumber $=6415385$ 
[86] C. Guthy, W. Utschick, and M. Honig, "Large system analysis of sum capacity in the Gaussian MIMO broadcast channel," IEEE J. Sel. Areas Commun., vol. 31, no. 2, pp. 149-159, 2013. [Online]. Available: http://ieeexplore.ieee.org/stamp/stamp.jsp?arnumber $=6415387$

[87] J. Zhang, C.-K. Wen, S. Jin, X. Gao, and K.-K. Wong, "On capacity of large-scale MIMO multiple access channels with distributed sets of correlated antennas," IEEE J. Sel. Areas Commun., vol. 31, no. 2, pp. 133-148, 2013. [Online]. Available: http://ieeexplore.ieee.org/stamp/stamp. jsp?arnumber $=6415386$

[88] H. Q. Ngo, E. Larsson, and T. Marzetta, "Energy and spectral efficiency of very large multiuser MIMO systems," IEEE Trans. Commun., vol. 61, no. 4, pp. 1436-1449, 2013. [Online]. Available: http://ieeexplore.ieee.org/stamp/stamp.jsp?arnumber $=6457363$

[89] H. Huh, S.-H. Moon, Y.-T. Kim, I. Lee, and G. Caire, "Multi-cell MIMO downlink with cell cooperation and fair scheduling: A large-system limit analysis," IEEE Trans. Inf. Theory, vol. 57, no. 12, pp. 7771-7786, 2011. [Online]. Available: http://ieeexplore.ieee.org/stamp/stamp.jsp?arnumber $=$ 6094252

[90] H. Huh, A. Tulino, and G. Caire, "Network MIMO with linear zero-forcing beamforming: Large system analysis, impact of channel estimation, and reduced-complexity scheduling," IEEE Trans. Inf. Theory, vol. 58, no. 5, pp. 2911-2934, 2012. [Online]. Available: http://ieeexplore.ieee.org/stamp/stamp.jsp?arnumber $=6095627$

[91] S. Mohammed and E. Larsson, "Per-antenna constant envelope precoding for large multi-user MIMO systems," IEEE Trans. Commun., vol. 61, no. 3, pp. 1059-1071, 2013. [Online]. Available: http://ieeexplore.ieee.org/stamp/ stamp.jsp?arnumber $=6451071$

[92] C. Studer and E. Larsson, "PAR-aware large-scale multi-user MIMO-OFDM downlink," IEEE J. Sel. Areas Commun., vol. 31, no. 2, pp. 303-313, 2013. [Online]. Available: http://ieeexplore.ieee.org/stamp/stamp.jsp?arnumber $=$ 6415400

[93] H. Papadopoulos, G. Caire, and S. A. Ramprashad, "Achieving large spectral efficiencies from MU-MIMO with tens of antennas: Locationadaptive TDD MU-MIMO design and user scheduling," in Signals, Systems and Computers (ASILOMAR), 2010 Conference Record of the Forty Fourth Asilomar Conference on, 2010, pp. 636-643. [Online]. Available: http://ieeexplore.ieee.org/stamp/stamp.jsp?arnumber $=5757639$

[94] C. Shepard, H. Yu, N. Anand, E. Li, T. Marzetta, R. Yang, and L. Zhong, "Argos: practical many-antenna base stations," in Proceedings of the 18th 
annual international conference on Mobile computing and networking, ser. Mobicom '12. New York, NY, USA: ACM, 2012, pp. 53-64. [Online]. Available: http://doi.acm.org/10.1145/2348543.2348553

[95] R. W. Chang, "Synthesis of band-limited orthogonal signals for multichannel data transmission," Bell System Technical Journal, vol. 45, no. 10, pp. 17751796, 1966. [Online]. Available: http://dx.doi.org/10.1002/j.1538-7305.1966. tb02435.x

[96] B. Saltzberg, "Performance of an efficient parallel data transmission system," IEEE Trans. Commun. Technol., vol. 15, no. 6, pp. 805-811, 1967. [Online]. Available: http://ieeexplore.ieee.org/stamp/stamp.jsp?arnumber $=1089674$

[97] S. Weinstein and P. Ebert, "Data transmission by frequency-division multiplexing using the discrete Fourier transform," IEEE Trans. Commun. Technol., vol. 19, no. 5, pp. 628-634, 1971. [Online]. Available: http://ieeexplore.ieee.org/stamp/stamp.jsp?arnumber $=1090705$

[98] L. Hanzo, Y. Akhtman, L. Wang, and M. Jiang, MIMO-OFDM for LTE, WiFi and WiMAX: Coherent versus non-coherent and cooperative turbo transceivers. Wiley, 2011, vol. 9.

[99] E. Dahlman, S. Parkvall, and J. Skold, 4G: LTE/LTE-Advanced for Mobile Broadband: LTE/LTE-Advanced for Mobile Broadband. Academic Press, 2011.

[100] H. Sampath, S. Talwar, J. Tellado, V. Erceg, and A. Paulraj, "A fourth-generation MIMO-OFDM broadband wireless system: design, performance, and field trial results," IEEE Commun. Mag., vol. 40, no. 9, pp. 143-149, 2002. [Online]. Available: http://ieeexplore.ieee.org/stamp/ stamp.jsp?arnumber $=1031841$

[101] H. Bolcskei, D. Gesbert, and A. Paulraj, "On the capacity of OFDM-based spatial multiplexing systems," IEEE Trans. Commun., vol. 50, no. 2, pp. 225-234, 2002. [Online]. Available: http://ieeexplore.ieee.org/stamp/stamp. jsp?arnumber $=983319$

[102] C. Oestges and B. Clerckx, MIMO wireless communications: from real-world propagation to space-time code design. Academic Press, 2007.

[103] R. Heath, D. Love, B. Rao, V. Lau, D. Gesbert, and M. Andrews, "Exploiting limited feedback in tomorrow's wireless communication networks," IEEE J. Sel. Areas Commun., vol. 26, no. 8, pp. 1337-1340, 2008. [Online]. Available: http://ieeexplore.ieee.org/stamp/stamp.jsp?arnumber $=4641945$

[104] M. Rupp, A. Perez-Neira, D. Gesbert, and C. Mecklenbrauker, "MIMO transmission with limited feedback," EURASIP Journal on Advances in 
Signal Processing, vol. 2008, no. 1, p. 518950, 2008. [Online]. Available: http://asp.eurasipjournals.com/content/2008/1/518950

[105] E. Visotsky and U. Madhow, "Space-time transmit precoding with imperfect feedback," IEEE Trans. Inf. Theory, vol. 47, no. 6, pp. 2632-2639, 2001. [Online]. Available: http://ieeexplore.ieee.org/stamp/stamp.jsp?arnumber $=$ 945281

[106] H. Zhang, S. Wei, G. Ananthaswamy, and D. Goeckel, "Adaptive signaling based on statistical characterizations of outdated feedback in wireless communications," Proc. IEEE, vol. 95, no. 12, pp. 2337-2353, 2007. [Online]. Available: http://ieeexplore.ieee.org/stamp/stamp.jsp?arnumber $=4389762$

[107] V. Lau, Y. Liu, and T.-A. Chen, "On the design of MIMO blockfading channels with feedback-link capacity constraint," IEEE Trans. Commun., vol. 52, no. 1, pp. 62-70, 2004. [Online]. Available: http://ieeexplore.ieee.org/stamp/stamp.jsp?arnumber $=1264195$

[108] A. Dabbagh and D. Love, "Feedback rate-capacity loss tradeoff for limited feedback MIMO systems," IEEE Trans. Inf. Theory, vol. 52, no. 5, pp. 2190-2202, 2006. [Online]. Available: http: //ieeexplore.ieee.org/stamp/stamp.jsp?arnumber $=1624652$

[109] B. Banister and J. Zeidler, "Feedback assisted transmission subspace tracking for MIMO systems," IEEE J. Sel. Areas Commun., vol. 21, no. 3, pp. 452-463, 2003. [Online]. Available: http://ieeexplore.ieee.org/stamp/ stamp.jsp?arnumber $=1192182$

[110] B. Mondal and R. W. Heath, "Channel adaptive quantization for limited feedback MIMO beamforming systems," IEEE Trans. Signal Process., vol. 54, no. 12, pp. 4717-4729, 2006. [Online]. Available: http://ieeexplore.ieee.org/stamp/stamp.jsp?arnumber $=4014385$

[111] S. Jafar and A. Goldsmith, "Transmitter optimization and optimality of beamforming for multiple antenna systems," IEEE Trans. Wireless Commun., vol. 3, no. 4, pp. 1165-1175, 2004. [Online]. Available: http://ieeexplore.ieee.org/stamp/stamp.jsp?arnumber $=1310307$

[112] S. Srinivasa and S. Jafar, "The optimality of transmit beamforming: A unified view," IEEE Trans. Inf. Theory, vol. 53, no. 4, pp. 1558-1564, 2007. [Online]. Available: http://ieeexplore.ieee.org/stamp/stamp.jsp?arnumber $=$ 4137899

[113] S. Thoen, L. Van der Perre, B. Gyselinckx, and M. Engels, "Performance analysis of combined transmit-SC/receive-MRC," IEEE Trans. Commun., vol. 49, no. 1, pp. 5-8, 2001. [Online]. Available: http://ieeexplore.ieee.org/ stamp/stamp.jsp?arnumber $=898241$ 
[114] M. Simon and M. Alouini, Digital Communication over Fading Channels, ser. Wiley Series in Telecommunications and Signal Processing. WileyInterscience, 2005.

[115] R. Heath and A. Paulraj, "A simple scheme for transmit diversity using partial channel feedback," in Signals, Systems $\&$ Computers, 1998. Conference Record of the Thirty-Second Asilomar Conference on, vol. 2, 1998, pp. 1073-1078. [Online]. Available: http://ieeexplore.ieee.org/stamp/ stamp.jsp?arnumber $=751427$

[116] A. Narula, M. Lopez, M. Trott, and G. W. Wornell, "Efficient use of side information in multiple-antenna data transmission over fading channels," IEEE J. Sel. Areas Commun., vol. 16, no. 8, pp. 1423-1436, 1998. [Online]. Available: http://ieeexplore.ieee.org/stamp/stamp.jsp?arnumber $=730451$

[117] D. J. Love, R. W. Heath, Jr., W. Santipach, and M. L. Honig, "What is the value of limited feedback for MIMO channels?" IEEE Commun. Mag., vol. 42, no. 10, pp. 54-59, 2004. [Online]. Available: http://ieeexplore.ieee.org/stamp/stamp.jsp?arnumber $=1341261$

[118] D. Love and R. Heath, "Equal gain transmission in multiple-input multiple-output wireless systems," IEEE Trans. Commun., vol. 51, no. 7, pp. 1102-1110, 2003. [Online]. Available: http://ieeexplore.ieee.org/stamp/ stamp.jsp?arnumber $=1214832$

[119] C. Murthy and B. Rao, "Quantization methods for equal gain transmission with finite rate feedback," IEEE Trans. Signal Process., vol. 55, no. 1, pp. 233-245, 2007. [Online]. Available: http://ieeexplore.ieee.org/stamp/stamp. jsp?arnumber $=4034215$

[120] D. J. Love, R. W. Heath, Jr., and T. Strohmer, "Grassmannian beamforming for multiple-input multiple-output wireless systems," IEEE Trans. Inf. Theory, vol. 49, no. 10, pp. 2735-2747, 2003. [Online]. Available: http://ieeexplore.ieee.org/stamp/stamp.jsp?arnumber $=1237152$

[121] V. Raghavan, R. W. Heath, and A. V. Sayeed M., "Systematic codebook designs for quantized beamforming in correlated MIMO channels," IEEE J. Sel. Areas Commun., vol. 25, no. 7, pp. 1298-1310, 2007. [Online]. Available: http://ieeexplore.iee.org/stamp/stamp.jsp?arnumber $=4299601$

[122] J. Roh and B. Rao, "Transmit beamforming in multiple-antenna systems with finite rate feedback: a VQ-based approach," IEEE Trans. Inf. Theory, vol. 52, no. 3, pp. 1101-1112, 2006. [Online]. Available: http://ieeexplore.ieee.org/stamp/stamp.jsp?arnumber $=1603774$

[123] P. Xia and G. Giannakis, "Design and analysis of transmit-beamforming based on limited-rate feedback," IEEE Trans. Signal Process., vol. 54, no. 5, 
pp. 1853-1863, 2006. [Online]. Available: http://ieeexplore.ieee.org/stamp/ stamp.jsp?arnumber $=1621413$

[124] J. Zheng, E. Duni, and B. Rao, "Analysis of multiple-antenna systems with finite-rate feedback using high-resolution quantization theory," IEEE Trans. Signal Process., vol. 55, no. 4, pp. 1461-1476, 2007. [Online]. Available: http://ieeexplore.ieee.org/stamp/stamp.jsp?arnumber $=4133055$

[125] J. Zheng and B. Rao, "Analysis of multiple antenna systems with finite-rate channel information feedback over spatially correlated fading channels," IEEE Trans. Signal Process., vol. 55, no. 9, pp. 4612-4626, 2007. [Online]. Available: http://ieeexplore.ieee.org/stamp/stamp.jsp?arnumber $=4291881$

[126] W. Santipach and M. Honig, "Asymptotic capacity of beamforming with limited feedback," in Information Theory, 2004. ISIT 2004. Proceedings. International Symposium on, 2004. [Online]. Available: http://ieeexplore.ieee.org/stamp/stamp.jsp?arnumber $=1365326$

[127] C. K. Au-Yeung and D. J. Love, "On the performance of random vector quantization limited feedback beamforming in a MISO system," IEEE Trans. Wireless Commun., vol. 6, no. 2, pp. 458-462, 2007. [Online]. Available: http://ieeexplore.ieee.org/stamp/stamp.jsp?arnumber $=4100151$

[128] C. Jotten, P. Baier, M. Meurer, T. Weber, and M. Haardt, "Efficient representation and feedback signaling of channel state information in frequency division duplexing MIMO systems," in Wireless Personal Multimedia Communications, 2002. The 5th International Symposium on, vol. 2, 2002, pp. 444-448. [Online]. Available: http://ieeexplore.ieee.org/ stamp/stamp.jsp?arnumber $=1088213$

[129] S. Sanayei and A. Nosratinia, "Antenna selection in MIMO systems," IEEE Commun. Mag., vol. 42, no. 10, pp. 68-73, 2004. [Online]. Available: http://ieeexplore.ieee.org/stamp/stamp.jsp?arnumber $=1341263$

[130] R. Heath and D. Love, "Multimode antenna selection for spatial multiplexing systems with linear receivers," IEEE Trans. Signal Process., vol. 53, no. 8, pp. 3042-3056, 2005. [Online]. Available: http: //ieeexplore.ieee.org/stamp/stamp.jsp?arnumber $=1468498$

[131] D. Love and R. Heath, "Limited feedback unitary precoding for spatial multiplexing systems," IEEE Trans. Inf. Theory, vol. 51, no. 8, pp. 2967-2976, 2005. [Online]. Available: http://ieeexplore.ieee.org/stamp/ stamp.jsp?arnumber $=1468321$

[132] W. Santipach and M. Honig, "Achievable rates for MIMO fading channels with limited feedback," in Spread Spectrum Techniques and Applications, 2004 IEEE Eighth International Symposium on, 2004, pp. 1-6. [Online]. Available: http://ieeexplore.ieee.org/stamp/stamp.jsp?arnumber $=1371652$ 
[133] — - "Asymptotic performance of MIMO wireless channels with limited feedback," in Military Communications Conference, 2003. MILCOM '03. 2003 IEEE, vol. 1, 2003, pp. 141-146. [Online]. Available: http://ieeexplore.ieee.org/stamp/stamp.jsp?arnumber $=1290092$

[134] J. Choi and R. W. Heath, Jr., "Interpolation based transmit beamforming for MIMO-OFDM with limited feedback," IEEE Trans. Signal Process., vol. 53, no. 11, pp. 4125-4135, 2005. [Online]. Available: http: //ieeexplore.ieee.org/stamp/stamp.jsp?arnumber $=1519681$

[135] J. Choi, B. Mondal, and R. W. Heath, "Interpolation based unitary precoding for spatial multiplexing MIMO-OFDM with limited feedback," IEEE Trans. Signal Process., vol. 54, no. 12, pp. 4730-4740, 2006. [Online]. Available: http://ieeexplore.ieee.org/stamp/stamp.jsp?arnumber $=4014368$

[136] T. Pande, D. Love, and J. Krogmeier, "A weighted least squares approach to precoding with pilots for MIMO-OFDM," IEEE Trans. Signal Process., vol. 54, no. 10, pp. 4067-4073, 2006. [Online]. Available: http://ieeexplore.ieee.org/stamp/stamp.jsp?arnumber $=1703875$

[137] — - "Reduced feedback MIMO-OFDM precoding and antenna selection," IEEE Trans. Signal Process., vol. 55, no. 5, pp. 2284-2293, 2007. [Online]. Available: http://ieeexplore.ieee.org/stamp/stamp.jsp?arnumber $=4160202$

[138] R. Grunheid, E. Bolinth, and H. Rohling, "A blockwise loading algorithm for the adaptive modulation technique in OFDM systems," in Vehicular Technology Conference, 2001. VTC 2001 Fall. IEEE VTS 54th, vol. 2, 2001, pp. 948-951. [Online]. Available: http: //ieeexplore.ieee.org/stamp/stamp.jsp?arnumber $=956913$

[139] P. Ding, D. Love, and M. Zoltowski, "Multiple antenna broadcast channels with limited feedback," in Acoustics, Speech and Signal Processing, 2006. ICASSP 2006 Proceedings. 2006 IEEE International Conference on, vol. 4, 2006. [Online]. Available: http://ieeexplore.ieee.org/stamp/stamp. jsp?arnumber $=1660896$

[140] D. Gerlach and A. Paulraj, "Adaptive transmitting antenna arrays with feedback," IEEE Signal Process. Lett., vol. 1, no. 10, pp. 150-152, 1994. [Online]. Available: http://ieeexplore.ieee.org/stamp/stamp.jsp?arnumber $=$ 329842

[141] N. Jindal, "MIMO broadcast channels with finite-rate feedback," IEEE Trans. Inf. Theory, vol. 52, no. 11, pp. 5045-5060, 2006. [Online]. Available: http://ieeexplore.ieee.org/stamp/stamp.jsp?arnumber $=1715541$

[142] P. Ding, D. Love, and M. Zoltowski, "Multiple antenna broadcast channels with shape feedback and limited feedback," IEEE Trans. Signal 
Process., vol. 55, no. 7, pp. 3417-3428, 2007. [Online]. Available: http://ieeexplore.ieee.org/stamp/stamp.jsp?arnumber $=4244670$

[143] A. Dabbagh and D. Love, "Multiple antenna MMSE based downlink precoding with quantized feedback or channel mismatch," IEEE Trans. Commun., vol. 56, no. 11, pp. 1859-1868, 2008. [Online]. Available: http://ieeexplore.ieee.org/stamp/stamp.jsp?arnumber $=4686268$

[144] G. Caire, N. Jindal, M. Kobayashi, and N. Ravindran, "Multiuser MIMO achievable rates with downlink training and channel state feedback," IEEE Trans. Inf. Theory, vol. 56, no. 6, pp. 2845-2866, 2010. [Online]. Available: http://ieeexplore.ieee.org/stamp/stamp.jsp?arnumber $=5466522$

[145] G. Dietl and G. Bauch, "Linear precoding in the downlink of limited feedback multiuser MIMO systems," in Global Telecommunications Conference, 200\%. GLOBECOM '07. IEEE, 2007, pp. 4359-4364. [Online]. Available: http://ieeexplore.ieee.org/stamp/stamp.jsp?arnumber $=4411739$

[146] W. Dai, Y. Liu, B. Rider, and W. Gao, "How many users should be turned on in a multi-antenna broadcast channel?" IEEE J. Sel. Areas Commun., vol. 26, no. 8, pp. 1526-1535, 2008. [Online]. Available: http://ieeexplore.ieee.org/stamp/stamp.jsp?arnumber $=4641962$

[147] P. Castro, M. Joham, L. Castedo, and W. Utschick, "Optimized CSI feedback for robust THP design," in Signals, Systems and Computers, 200\%. ACSSC 200\%. Conference Record of the Forty-First Asilomar Conference on, 2007, pp. 1956-1960. [Online]. Available: http://ieeexplore.ieee.org/stamp/stamp.jsp?arnumber $=4487578$

[148] B. Clerckx, G. Kim, J. Choi, and Y.-J. Hong, "Explicit vs. implicit feedback for SU and MU-MIMO," in Global Telecommunications Conference (GLOBECOM 2010), 2010 IEEE, 2010, pp. 1-5. [Online]. Available: http://ieeexplore.ieee.org/stamp/stamp.jsp?arnumber $=5683816$

[149] M. Joham, P. Castro, W. Utschick, and L. Castedo, "Robust precoding with limited feedback design based on precoding MSE for MU-MISO systems," IEEE Trans. Signal Process., vol. 60, no. 6, pp. 3101-3111, 2012. [Online]. Available: http://ieeexplore.ieee.org/stamp/stamp.jsp?arnumber $=6144751$

[150] M. Sharif and B. Hassibi, "On the capacity of MIMO broadcast channels with partial side information," IEEE Trans. Inf. Theory, vol. 51, no. 2, pp. 506-522, 2005. [Online]. Available: http://ieeexplore.ieee.org/stamp/stamp. jsp?arnumber $=1386524$

[151] J. Diaz, O. Simeone, and Y. Bar-Ness, "Asymptotic analysis of reducedfeedback strategies for MIMO Gaussian broadcast channels," IEEE Trans. Inf. Theory, vol. 54, no. 3, pp. 1308-1316, 2008. [Online]. Available: http://ieeexplore.ieee.org/stamp/stamp.jsp?arnumber $=4455741$ 
[152] W. Zhang and K. Letaief, "MIMO broadcast scheduling with limited feedback," IEEE J. Sel. Areas Commun., vol. 25, no. 7, pp. 1457-1467, 2007. [Online]. Available: http://ieeexplore.ieee.org/stamp/stamp.jsp?arnumber= 4299615

[153] M. Kountouris, T. Salzer, and D. Gesbert, "Scheduling for multiuser MIMO downlink channels with ranking-based feedback," EURASIP Journal on Advances in Signal Processing, vol. 2008, no. 1, p. 854120, 2008. [Online]. Available: http://asp.eurasipjournals.com/content/2008/1/854120

[154] T. Yoo, N. Jindal, and A. Goldsmith, "Multi-antenna downlink channels with limited feedback and user selection," IEEE J. Sel. Areas Commun., vol. 25, no. 7, pp. 1478-1491, 2007. [Online]. Available: http://ieeexplore.ieee.org/stamp/stamp.jsp?arnumber $=4299617$

[155] M. Kountouris, D. Gesbert, and T. Salzer, "Enhanced multiuser random beamforming: dealing with the not so large number of users case," IEEE J. Sel. Areas Commun., vol. 26, no. 8, pp. 1536-1545, 2008. [Online]. Available: http://ieeexplore.ieee.org/stamp/stamp.jsp?arnumber $=4641963$

[156] K. Huang, J. Andrews, and R. Heath, "Performance of orthogonal beamforming for SDMA with limited feedback," IEEE Trans. Veh. Technol., vol. 58, no. 1, pp. 152-164, 2009. [Online]. Available: http://ieeexplore.ieee.org/stamp/stamp.jsp?arnumber $=4510711$

[157] P. Svedman, S. Wilson, L. Cimini, and B. Ottersten, "Opportunistic beamforming and scheduling for OFDMA systems," IEEE Trans. Commun., vol. 55, no. 5, pp. 941-952, 2007. [Online]. Available: http://ieeexplore.ieee.org/stamp/stamp.jsp?arnumber $=4200961$

[158] I. Toufik and H. Kim, "MIMO-OFDMA opportunistic beamforming with partial channel state information," in Communications, 2006. ICC '06. IEEE International Conference on, vol. 12, 2006, pp. 5389-5394. [Online]. Available: http://ieeexplore.ieee.org/stamp/stamp.jsp?arnumber $=4024918$

[159] J. Sanchez-Garcia, L. Soriano-Equigua, and R. Heath, "Quantized antenna combining for multiuser MIMO-OFDM with limited feedback," IEEE Signal Process. Lett., vol. 16, no. 12, pp. 1027-1030, 2009. [Online]. Available: http://ieeexplore.ieee.org/stamp/stamp.jsp?arnumber $=5184938$

[160] M. Trivellato, S. Tomasin, and N. Benvenuto, "On channel quantization and feedback strategies for multiuser MIMO-OFDM downlink systems," IEEE Trans. Commun., vol. 57, no. 9, pp. 2645-2654, 2009. [Online]. Available: http://ieeexplore.ieee.org/stamp/stamp.jsp?arnumber $=5281754$

[161] H. Shirani-Mehr and G. Caire, "Channel state feedback schemes for multiuser MIMO-OFDM downlink," IEEE Trans. Commun., vol. 57, no. 9, 
pp. 2713-2723, 2009. [Online]. Available: http://ieeexplore.ieee.org/stamp/ stamp.jsp?arnumber $=5281760$

[162] P. de Kerret and D. Gesbert, "CSI sharing strategies for transmitter cooperation in wireless networks," IEEE Wireless Commun. Mag., vol. 20, no. 1, pp. 43-49, 2013. [Online]. Available: http://ieeexplore.ieee.org/ stamp/stamp.jsp?arnumber $=6472198$

[163] P. Marsch and G. Fettweis, "A framework for optimizing the uplink performance of distributed antenna systems under a constrained backhaul," in Communications, 200\%. ICC '0\%. IEEE International Conference on, 2007, pp. 975-979. [Online]. Available: http://ieeexplore.ieee.org/stamp/ stamp.jsp?arnumber $=4288836$

[164] A. Papadogiannis, D. Gesbert, and E. Hardouin, "A dynamic clustering approach in wireless networks with multi-cell cooperative processing," in Proc. IEEE Int. Conf. Communications ICC '08, 2008, pp. 4033-4037. [Online]. Available: http://ieeexplore.ieee.org/stamp/stamp.jsp?arnumber $=$ 4533793

[165] J. Zhang, R. Chen, J. Andrews, A. Ghosh, and R. Heath, "Networked MIMO with clustered linear precoding," IEEE Trans. Wireless Commun., vol. 8, no. 4, pp. 1910-1921, 2009. [Online]. Available: http: //ieeexplore.ieee.org/stamp/stamp.jsp?arnumber $=4907459$

[166] P. Marsch and G. Fettweis, "Static clustering for cooperative multi-point (CoMP) in mobile communications," in Communications (ICC), 2011 IEEE International Conference on, 2011, pp. 1-6. [Online]. Available: http://ieeexplore.ieee.org/stamp/stamp.jsp?arnumber $=5963458$

[167] — , "Uplink CoMP under a constrained backhaul and imperfect channel knowledge," IEEE Trans. Wireless Commun., vol. 10, no. 6, pp. 1730 1742, 2011. [Online]. Available: http://ieeexplore.ieee.org/stamp/stamp. jsp?arnumber $=5754752$

[168] J. Kim, W. Zirwas, and M. Haardt, "Efficient feedback via subspace-based channel quantization for distributed cooperative antenna systems with temporally correlated channels," EURASIP Journal on Advances in Signal Processing, vol. 2008, no. 1, p. 847296, 2008. [Online]. Available: http://asp.eurasipjournals.com/content/2008/1/847296

[169] A. Papadogiannis, H. Bang, D. Gesbert, and E. Hardouin, "Efficient selective feedback design for multicell cooperative networks," IEEE Trans. Veh. Technol., vol. 60, no. 1, pp. 196-205, 2011. [Online]. Available: http://ieeexplore.ieee.org/stamp/stamp.jsp?arnumber $=5609224$ 
[170] W. Ho, T. Quek, S. Sun, and R. Heath, "Decentralized precoding for multicell MIMO downlink," IEEE Trans. Wireless Commun., vol. 10, no. 6, pp. 1798-1809, 2011. [Online]. Available: http: //ieeexplore.ieee.org/stamp/stamp.jsp?arnumber $=5751591$

[171] K. Yu and B. Ottersten, "Models for MIMO propagation channels: a review," Wireless Communications and Mobile Computing, vol. 2, no. 7, pp. 653-666, 2002. [Online]. Available: http://onlinelibrary.wiley.com/doi/ $10.1002 /$ wcm.78/full

[172] P. Almers, E. Bonek, A. Burr, N. Czink, M. Debbah, V. Degli-Esposti, H. Hofstetter, P. Kyö, D. Laurenson, G. Matz et al., "Survey of channel and radio propagation models for wireless MIMO systems," EURASIP Journal on Wireless Communications and Networking, vol. 2007, 2007. [Online]. Available: http://jwcn.eurasipjournals.com/content/2007/1/019070

[173] M. Debbah and R. Muller, "MIMO channel modeling and the principle of maximum entropy," IEEE Trans. Inf. Theory, vol. 51, no. 5, pp. 1667-1690, 2005. [Online]. Available: http://ieeexplore.ieee.org/stamp/ stamp.jsp?arnumber $=1424308$

[174] D. shan Shiu, G. J. Foschini, M. J. Gans, and J. M. Kahn, "Fading correlation and its effect on the capacity of multielement antenna systems," IEEE Trans. Commun., vol. 48, no. 3, pp. 502-513, 2000. [Online]. Available: http://ieeexplore.ieee.org/stamp/stamp.jsp?arnumber $=837052$

[175] J. Kermoal, L. Schumacher, K. Pedersen, P. Mogensen, and F. Frederiksen, "A stochastic MIMO radio channel model with experimental validation," IEEE J. Sel. Areas Commun., vol. 20, no. 6, pp. 1211-1226, 2002. [Online]. Available: http://ieeexplore.ieee.org/stamp/stamp.jsp?arnumber $=1021913$

[176] F. Domene, G. Pinero, M. de Diego, and A. Gonzalez, "Channel quantization based on the statistical characterization of spatially-correlated fading," IEEE Trans. Veh. Technol., to be published, early Access. [Online]. Available: http://ieeexplore.ieee.org/stamp/stamp.jsp?arnumber $=6924760$

[177] H. Ozcelik, M. Herdin, W. Weichselberger, J. Wallace, and E. Bonek, "Deficiencies of 'Kronecker' MIMO radio channel model," Electronics Letters, vol. 39, no. 16, pp. 1209-1210, 2003. [Online]. Available: http: //ieeexplore.ieee.org/stamp/stamp.jsp?arnumber $=1226586$

[178] V. Raghavan, J. H. Kotecha, and A. Sayeed, "Why does the Kronecker model result in misleading capacity estimates?" IEEE Trans. Inf. Theory, vol. 56, no. 10, pp. 4843-4864, 2010. [Online]. Available: http://ieeexplore.ieee.org/stamp/stamp.jsp?arnumber $=5571876$

[179] Rec. ITU-R M.1225, "Guidelines for evaluation of radio transmission technologies for IMT-2000," 1997. 
[180] 3GPP TS 05.05 V8.20.0, "Radio transmission and reception (release 1999)."

[181] Ericsson, Nokia, Motorola, Rohde \& Schwarz, "R4-070572: Proposal for LTE channel models," May 2007, kobe, Japan.

[182] 3GPP TR 25.996 V10.0.0, "Spatial channel model for multiple input multiple output (MIMO) simulations."

[183] C.-X. Wang, X. Hong, H. Wu, and W. Xu, "Spatial-temporal correlation properties of the $3 \mathrm{GPP}$ spatial channel model and the Kronecker MIMO channel model," EURASIP Journal on Wireless Communications and Networking, vol. 2007, no. 1, p. 039871, 2007. [Online]. Available: http://jwcn.eurasipjournals.com/content/2007/1/039871

[184] D. S. Baum, J. Hansen, and J. Salo, "An interim channel model for beyond-3G systems: extending the 3GPP spatial channel model (SCM)," in Proc. VTC 2005-Spring Vehicular Technology Conf. 2005 IEEE 61st, vol. 5, 2005, pp. 3132-3136. [Online]. Available: http: //ieeexplore.ieee.org/stamp/stamp.jsp?arnumber $=1543924$

[185] M. Narandzic, C. Schneider, R. Thoma, T. Jamsa, P. Kyosti, and X. Zhao, "Comparison of SCM, SCME, and WINNER channel models," in Vehicular Technology Conference, 200\%. VTC2007-Spring. IEEE 65th, 2007, pp. 413-417. [Online]. Available: http://ieeexplore.ieee.org/stamp/stamp.jsp? arnumber $=4212524$

[186] D. Wubben, D. Seethaler, J. Jalden, and G. Matz, "Lattice reduction," IEEE Signal Process. Mag., vol. 28, no. 3, pp. 70-91, 2011. [Online]. Available: http://ieeexplore.ieee.org/xpl/articleDetails.jsp?arnumber $=5753113$

[187] P. Q. Nguyen and B. Vallée, The LLL Algorithm. Springer, 2010.

[188] M. R. Bremner, Lattice basis reduction: An introduction to the LLL algorithm and its applications. CRC Press, 2012.

[189] B. Helfrich, "Algorithms to construct minkowski reduced and hermite reduced lattice bases," Theoretical Computer Science, vol. 41, pp. 125-139, 1985. [Online]. Available: http://www.sciencedirect.com/science/article/ pii/0304397585900672

[190] M. Grötschel, L. Lovász, and A. Schrijver, Geometric algorithms and combinatorial optimization. Springer-Verlag, 1988.

[191] H. Cohen, A course in computational algebraic number theory. Springer, 1993, vol. 138 . 
[192] S. Roger, F. Domene, A. Gonzalez, V. Almenar, and G. Piñero, "An evaluation of precoding techniques for multiuser communication systems," in Proc. 7th Int Wireless Communication Systems (ISWCS) Symp, 2010, pp. 295-299. [Online]. Available: http://ieeexplore.ieee.org/stamp/stamp. jsp?arnumber $=5624548$

[193] B. Hochwald and S. Vishwanath, "Space-time multiple access: Linear growth in the sum rate," in Proc. 40th Annual Allerton Conf. Communications, Control and Computing, 2002.

[194] A. Wiesel, Y. Eldar, and S. Shamai, "Zero-forcing precoding and generalized inverses," IEEE Trans. Signal Process., vol. 56, no. 9, pp. 4409-4418, 2008. [Online]. Available: http://ieeexplore.ieee.org/stamp/stamp.jsp?arnumber $=$ 4599181

[195] D. Bartolome and A. Perez-Neira, "Spatial scheduling in multiuser wireless systems: from power allocation to admission control," IEEE Trans. Wireless Commun., vol. 5, no. 8, pp. 2082-2091, 2006. [Online]. Available: http://ieeexplore.ieee.org/stamp/stamp.jsp?arnumber $=1687723$

[196] T. Yoo and A. Goldsmith, "On the optimality of multiantenna broadcast scheduling using zero-forcing beamforming," IEEE J. Sel. Areas Commun., vol. 24, no. 3, pp. 528-541, 2006. [Online]. Available: http://ieeexplore.ieee.org/stamp/stamp.jsp?arnumber $=1603708$

[197] U. Fincke and M. Pohst, "Improved methods for calculating vectors of short length in a lattice, including a complexity analysis," Mathematics of computation, vol. 44, no. 170, pp. 463-471, 1985. [Online]. Available: http://www.ams.org/mcom/1985-44-170/S0025-5718-1985-0777278-8/

[198] O. Damen, A. Chkeif, and J.-C. Belfiore, "Lattice code decoder for spacetime codes," IEEE Commun. Lett., vol. 4, no. 5, pp. 161-163, 2000. [Online]. Available: http://ieeexplore.ieee.org/stamp/stamp.jsp?arnumber $=846498$

[199] E. Agrell, T. Eriksson, A. Vardy, and K. Zeger, "Closest point search in lattices," IEEE Trans. Inf. Theory, vol. 48, no. 8, pp. 2201-2214, 2002. [Online]. Available: http://ieeexplore.ieee.org/stamp/stamp.jsp?arnumber= 1019833

[200] B. Hassibi and H. Vikalo, "On the sphere-decoding algorithm I. expected complexity," IEEE Trans. Signal Process., vol. 53, no. 8, pp. 28062818, 2005. [Online]. Available: http://ieeexplore.ieee.org/stamp/stamp. jsp?arnumber $=1468474$

[201] R. Wesel and J. Cioffi, "Achievable rates for Tomlinson-Harashima precoding," IEEE Trans. Inf. Theory, vol. 44, no. 2, pp. 824-831, 1998. [Online]. Available: http://ieeexplore.ieee.org/stamp/stamp.jsp?arnumber= 661530 
[202] R. F. Fischer, C. Windpassinger, A. Lampe, and J. B. Huber, "Space-time transmission using Tomlinson-Harashima precoding," in Proc. 4th Int. ITG Conf., 2002, pp. 139-147.

[203] M. Payaró, A. Perez-Neira, and M.-A. Lagunas, "Achievable rates for generalized spatial Tomlinson-Harashima precoding in MIMO systems," in Vehicular Technology Conference, 2004. VTC2004-Fall. 2004 IEEE 60th, vol. 4, 2004, pp. 2462-2466. [Online]. Available: http://ieeexplore.ieee.org/ stamp/stamp.jsp?arnumber $=1400496$

[204] R. F. H. Fischer, C. Windpassinger, A. Lampe, and J. Huber, "MIMO precoding for decentralized receivers," in Information Theory, 2002. Proceedings. 2002 IEEE International Symposium on, 2002. [Online]. Available: http://ieeexplore.ieee.org/stamp/stamp.jsp?arnumber $=1023768$

[205] C. Windpassinger, "Detection and precoding for multiple input multiple output channels," Ph.D. dissertation, University Erlangen-Nurnberg, Germany, 2004.

[206] C.-H. Fung, W. Yu, and T. J. Lim, "Precoding for the multiantenna downlink: Multiuser SNR gap and optimal user ordering," IEEE Trans. Commun., vol. 55, no. 1, pp. 188-197, 2007. [Online]. Available: http://ieeexplore.ieee.org/stamp/stamp.jsp?arnumber $=4063503$

[207] H. Yao and G. W. Wornell, "Lattice-reduction-aided detectors for MIMO communication systems," in Proc. IEEE Global Telecommunications Conf. GLOBECOM '02, vol. 1, 2002, pp. 424-428. [Online]. Available: http://ieeexplore.ieee.org/stamp/stamp.jsp?arnumber $=1188114$

[208] D. Wubben, R. Bohnke, V. Kuhn, and K.-D. Kammeyer, "Near-maximumlikelihood detection of MIMO systems using MMSE-based lattice reduction," in Proc. IEEE Int Communications Conf, vol. 2, 2004, pp. 798-802. [Online]. Available: http://ieeexplore.iee.org/stamp/stamp.jsp?arnumber $=1312611$

[209] L. Babai, "On Lovász' lattice reduction and the nearest lattice point problem," Combinatorica, vol. 6, no. 1, pp. 1-13, 1986. [Online]. Available: http://link.springer.com/article/10.1007/BF02579403

[210] X. Ma and W. Zhang, "Performance analysis for MIMO systems with lattice-reduction aided linear equalization," IEEE Trans. Commun., vol. 56, no. 2, pp. 309-318, 2008. [Online]. Available: http://ieeexplore.ieee.org/ stamp/stamp.jsp?arnumber $=4450801$

[211] Y. H. Gan, C. Ling, and W. H. Mow, "Complex lattice reduction algorithm for low-complexity full-diversity MIMO detection," IEEE Trans. Signal Process., vol. 57, no. 7, pp. 2701-2710, 2009. [Online]. Available: http://ieeexplore.ieee.org/stamp/stamp.jsp?arnumber $=4787140$ 
[212] M. Taherzadeh, A. Mobasher, and A. K. Khandani, "Communication over MIMO broadcast channels using lattice-basis reduction," IEEE Trans. Inf. Theory, vol. 53, no. 12, pp. 4567-4582, 2007. [Online]. Available: http://ieeexplore.ieee.org/stamp/stamp.jsp?arnumber $=4385786$

[213] G. Dimic and N. D. Sidiropoulos, "On downlink beamforming with greedy user selection: performance analysis and a simple new algorithm," IEEE Trans. Signal Process., vol. 53, no. 10, pp. 3857-3868, 2005. [Online]. Available: http://ieeexplore.ieee.org/stamp/stamp.jsp?arnumber $=1510992$

[214] M. Joham and W. Utschick, "Ordered spatial Tomlinson-Harashima precoding," Smart antennas state-of-the-art, vol. 3, 2006, chapter 20. [Online]. Available: http://downloads.hindawi.com/books/9789775945099/ art20.pdf

[215] A. Goldsmith, Wireless Communications. New York, NY, USA: Cambridge University Press, 2005.

[216] L. Barbero and J. Thompson, "Fixing the complexity of the sphere decoder for MIMO detection," IEEE Trans. Wireless Commun., vol. 7, no. 6, pp. 2131-2142, 2008. [Online]. Available: http://ieeexplore.ieee.org/stamp/ stamp.jsp?arnumber $=4543065$

[217] G. H. Golub and C. F. Van Loan, Matrix computations (3rd ed.). Baltimore, MD, USA: Johns Hopkins University Press, 1996.

[218] H. Vetter, V. Ponnampalam, M. Sandell, and P. A. Hoeher, "Fixed complexity LLL algorithm," IEEE Trans. Signal Process., vol. 57, no. 4, pp. 1634-1637, 2009. [Online]. Available: http://ieeexplore.ieee.org/stamp/ stamp.jsp?arnumber $=4732316$

[219] S. Roger, A. Gonzalez, V. Almenar, and A. M. Vidal, "Extended LLL algorithm for efficient signal precoding in multiuser communication systems," IEEE Commun. Lett., vol. 14, no. 3, pp. 220-222, 2010. [Online]. Available: http://ieeexplore.ieee.org/stamp/stamp.jsp?arnumber $=5426588$

[220] F. Domene, G. Piñero, C. Botella, and A. Gonzalez, "A limited feedback scheme based on spatially correlated channels for coordinated multipoint systems," EURASIP Journal on Wireless Communications and Networking, vol. 2012, no. 1, p. 176, 2012. [Online]. Available: http://www.springerlink.com/index/W072166710155756.pdf

[221] R. Fischer, C. Windpassinger, A. Lampe, and J. Huber, "TomlinsonHarashima precoding in space-time transmission for low-rate backward channel," in Broadband Communications, 2002. Access, Transmission, Networking. 2002 International Zurich Seminar on, 2002, pp. 7-1. [Online]. Available: http://ieeexplore.iee.org/stamp/stamp.jsp?arnumber $=991747$ 
[222] R. Hunger, F. Dietrich, M. Joham, and W. Utschick, "Robust transmit zero-forcing filters," in Smart Antennas, 2004. ITG Workshop on, 2004, pp. 130-137. [Online]. Available: http://ieeexplore.ieee.org/stamp/stamp. jsp?arnumber $=1407659$

[223] M. Shenouda and T. Davidson, "Robust linear precoding for uncertain MISO broadcast channels," in Acoustics, Speech and Signal Processing, 2006. ICASSP 2006 Proceedings. 2006 IEEE International Conference on, vol. 4, 2006. [Online]. Available: http://ieeexplore.ieee.org/stamp/stamp. jsp?arnumber $=1660899$

[224] F. Dietrich, P. Breun, and W. Utschick, "Robust Tomlinson-Harashima precoding for the wireless broadcast channel," IEEE Trans. Signal Process., vol. 55, no. 2, pp. 631-644, 2007. [Online]. Available: http://ieeexplore.ieee.org/stamp/stamp.jsp?arnumber $=4063540$

[225] M. Shenouda and T. Davidson, "Tomlinson-Harashima precoding for broadcast channels with uncertainty," IEEE J. Sel. Areas Commun., vol. 25, no. 7, pp. 1380-1389, 2007. [Online]. Available: http: //ieeexplore.ieee.org/stamp/stamp.jsp?arnumber $=4299608$

[226] M. Huang, S. Zhou, and J. Wang, "Analysis of Tomlinson-Harashima precoding in multiuser MIMO systems with imperfect channel state information," IEEE Trans. Veh. Technol., vol. 57, no. 5, pp. 28562867, 2008. [Online]. Available: http://ieeexplore.ieee.org/stamp/stamp. jsp?arnumber $=4400049$

[227] L. Sun and M. Lei, "Quantized CSI-based Tomlinson-Harashima precoding in multiuser MIMO systems," IEEE Trans. Wireless Commun., vol. 12, no. 3, pp. 1118-1126, 2013. [Online]. Available: http: //ieeexplore.ieee.org/stamp/stamp.jsp?arnumber $=6410304$

[228] F. Domene, S. Roger, C. Ramiro, G. Piñero, and A. Gonzalez, "Efficient implementation of multiuser precoding algorithms on GPU for MIMO-OFDM systems," in XXVII Simposium Nacional de la Unión Científica Internacional de Radio, URSI, 2012.

[229] F. Domene, S. Roger, C. Ramiro, G. Pinero, and A. Gonzalez, "A reconfigurable GPU implementation for Tomlinson-Harashima precoding," in Acoustics, Speech and Signal Processing (ICASSP), 2012 IEEE International Conference on, 2012, pp. 1629-1632. [Online]. Available: http://ieeexplore.ieee.org/stamp/stamp.jsp?arnumber $=6288207$

[230] F. Domene, C. M. Józsa, G. Piñero, A. Gonzalez, and A. M. Vidal, "Performance analysis of a parallel lattice reduction algorithm on many-core architectures," in Proceedings of the 13th International Conference on Computational and Mathematical Methods in Science and Engineering, CMMSE, 2013. 
[231] C. M. Józsa, F. Domene, G. Pinero, A. Gonzalez, and A. M. Vidal, "Efficient GPU implementation of lattice-reduction-aided multiuser precoding," in Wireless Communication Systems (ISWCS 2013), Proceedings of the Tenth International Symposium on, 2013, pp. 1-5. [Online]. Available: http://ieeexplore.ieee.org/stamp/stamp.jsp?arnumber $=6629866$

[232] C. M. Józsa, F. Domene, A. M. Vidal, G. Piñero, and A. González, "High performance lattice reduction on heterogeneous computing platform," The Journal of Supercomputing, pp. 1-14, 2014. [Online]. Available: http://dx.doi.org/10.1007/s11227-014-1201-2

[233] S. Che, M. Boyer, J. Meng, D. Tarjan, J. W. Sheaffer, and K. Skadron, "A performance study of general-purpose applications on graphics processors using CUDA," Journal of parallel and distributed computing, vol. 68, no. 10, pp. 1370-1380, 2008. [Online]. Available: http://www.sciencedirect.com/science/article/pii/S0743731508000932

[234] J. Nickolls, I. Buck, M. Garland, and K. Skadron, "Scalable parallel programming with CUDA," Queue, vol. 6, no. 2, pp. 40-53, 2008. [Online]. Available: http://dl.acm.org/citation.cfm?id=1365500

[235] M. Arora, S. Nath, S. Mazumdar, S. Baden, and D. Tullsen, "Redefining the role of the CPU in the era of CPU-GPU integration," IEEE Micro, vol. 32, no. 6, pp. 4-16, 2012. [Online]. Available: http://ieeexplore.ieee.org/stamp/stamp.jsp?arnumber $=6266669$

[236] D. B. Kirk and W.-m. W. Hwu, Programming massively parallel processors: a hands-on approach. Morgan Kaufmann Publishers Inc., 2012.

[237] NVIDIA Corporation, "CUDA C Programming Guide," 2012. [Online]. Available: http://docs.nvidia.com/cuda/cuda-c-programming-guide/

[238] M. J. Atallah, Algorithms and theory of computation handbook. CRC press, 1998.

[239] M. Flynn, "Some computer organizations and their effectiveness," IEEE Trans. Comput., vol. C-21, no. 9, pp. 948-960, 1972. [Online]. Available: http://ieeexplore.ieee.org/stamp/stamp.jsp?arnumber $=5009071$

[240] 3GPP TS 36.211 V10.0.0, "Evolved universal terrestrial radio access (EUTRA); physical channels and modulation."

[241] M. Li, D. Novo, B. Bougard, C. Desset, A. Dejonghe, L. Van Der Perre, and F. Catthoor, "A system level algorithmic approach toward energy-aware SDR baseband implementations," in Proc. IEEE Int. Conf. Communications ICC '09, 2009, pp. 1-6. [Online]. Available: http://ieeexplore.ieee.org/stamp/stamp.jsp?arnumber $=5198680$ 
[242] D. Wu, J. Eilert, and D. Liu, "A programmable lattice-reduction aided detector for MIMO-OFDMA," in Circuits and Systems for Communications, 2008. ICCSC 2008. 4th IEEE International Conference on, 2008, pp. 293-297. [Online]. Available: http://ieeexplore.ieee.org/stamp/stamp.jsp? arnumber $=4536760$

[243] L. Barbero, D. Milliner, T. Ratnarajah, J. Barry, and C. Cowan, "Rapid prototyping of Clarkson's lattice reduction for MIMO detection," in Communications, 2009. ICC '09. IEEE International Conference on, 2009, pp. 1-5. [Online]. Available: http://ieeexplore.ieee.org/stamp/stamp.jsp? arnumber $=5199388$

[244] L. Bruderer, C. Studer, M. Wenk, D. Seethaler, and A. Burg, "VLSI implementation of a low-complexity LLL lattice reduction algorithm for MIMO detection," in Circuits and Systems (ISCAS), Proceedings of 2010 IEEE International Symposium on, 2010, pp. 3745-3748. [Online]. Available: http://ieeexplore.ieee.org/stamp/stamp.jsp?arnumber $=5537742$

[245] B. Gestner, W. Zhang, X. Ma, and D. Anderson, "Lattice reduction for MIMO detection: From theoretical analysis to hardware realization," IEEE Trans. Circuits Syst. I, vol. 58, no. 4, pp. 813-826, 2011. [Online]. Available: http://ieeexplore.ieee.org/stamp/stamp.jsp?arnumber $=5638606$

[246] M. Shabany, A. Youssef, and G. Gulak, "High-throughput 0.13- $\mu \mathrm{m}$ CMOS lattice reduction core supporting $880 \mathrm{Mb} / \mathrm{s}$ detection," IEEE Trans. VLSI Syst., vol. 21, no. 5, pp. 848-861, 2013. [Online]. Available: http://ieeexplore.ieee.org/stamp/stamp.jsp?arnumber $=6232467$

[247] S. Wetzel, "An efficient parallel block-reduction algorithm," in Algorithmic Number Theory. Springer, 1998, pp. 323-337.

[248] Y. Luo and S. Qiao, "A parallel LLL algorithm," in Proceedings of The Fourth International $C^{*}$ Conference on Computer Science and Software Engineering, 2011, pp. 93-101.

[249] W. Backes and S. Wetzel, "Parallel lattice basis reduction - the road to many-core," in High Performance Computing and Communications (HPCC), 2011 IEEE 13th International Conference on, 2011, pp. 417-424. [Online]. Available: http://ieeexplore.iee.org/stamp/stamp.jsp?arnumber $=6063020$

[250] U. Ahmad, A. Amin, M. Li, S. Pollin, L. Van Der Perre, and F. Catthoor, "Scalable block-based parallel lattice reduction algorithm for an SDR baseband processor," in Communications (ICC), 2011 IEEE International Conference on, 2011, pp. 1-5. [Online]. Available: http://ieeexplore.ieee.org/stamp/stamp.jsp?arnumber $=5963386$ 
[251] G. Villard, "Parallel lattice basis reduction," in Papers from the international symposium on Symbolic and algebraic computation, ser. ISSAC '92. New York, NY, USA: ACM, 1992, pp. 269-277. [Online]. Available: http://doi.acm.org/10.1145/143242.143327

[252] B. Chapman, G. Jost, and R. Van Der Pas, Using OpenMP: portable shared memory parallel programming. MIT press, 2008, vol. 10.

[253] NVIDIA Corporation, "CUDA Dynamic Parallelism Programming Guide," 2012. [Online]. Available: http://docs.nvidia.com/cuda/pdf/ CUDA_Dynamic_Parallelism_Programming_Guide.pdf

[254] B. Gestner, W. Zhang, X. Ma, and D. Anderson, "VLSI implementation of a lattice reduction algorithm for low-complexity equalization," in Circuits and Systems for Communications, 2008. ICCSC 2008. 4th IEEE International Conference on, 2008, pp. 643-647. [Online]. Available: http://ieeexplore.ieee.org/stamp/stamp.jsp?arnumber $=4536834$

[255] A. Burg, D. Seethaler, and G. Matz, "VLSI implementation of a lattice-reduction algorithm for multi-antenna broadcast precoding," in Circuits and Systems, 200\%. ISCAS 200\%. IEEE International Symposium on, 2007, pp. 673-676. [Online]. Available: http://ieeexplore.ieee.org/ stamp/stamp.jsp?arnumber $=4252724$

[256] F. Domene, G. Piñero, M. de Diego, and A. González, "Performance of multiuser MIMO-OFDM precoding techniques with quantized channel information," in Proc. 7th Int Wireless Communication Systems (ISWCS) Symp, 2010, pp. 56-60. [Online]. Available: http://ieeexplore.ieee.org/ stamp/stamp.jsp?arnumber $=5624376$

[257] F. Domene and G. Pinero, "A channel quantization scheme based on the Karhunen-Loève transform," in Vehicular Technology Conference (VTC Spring), 2013 IEEE 7rth, 2013, pp. 1-5. [Online]. Available: http://ieeexplore.ieee.org/stamp/stamp.jsp?arnumber $=6691816$

[258] R. Gray and D. Neuhoff, "Quantization," IEEE Transactions on Information Theory, vol. 44, no. 6, pp. 2325-2383, 1998. [Online]. Available: http://ieeexplore.ieee.org/stamp/stamp.jsp?arnumber $=720541$

[259] A. Gersho and R. M. Gray, Vector quantization and signal compression. Norwell, MA, USA: Kluwer Academic Publishers, 1991.

[260] S. Lloyd, "Least squares quantization in PCM," IEEE Trans. Inf. Theory, vol. 28, no. 2, pp. 129-137, 1982. [Online]. Available: http://ieeexplore.ieee.org/stamp/stamp.jsp?arnumber $=1056489$ 
[261] J. Max, "Quantizing for minimum distortion," IRE Transactions on Information Theory, vol. 6, no. 1, pp. 7-12, 1960. [Online]. Available: http://ieeexplore.ieee.org/stamp/stamp.jsp?arnumber $=1057548$

[262] C. Shannon, "A mathematical theory of communication," pp. 379423, 1948, bell System Technical Journal, The. [Online]. Available: http://ieeexplore.ieee.org/stamp/stamp.jsp?arnumber $=6773024$

[263] C. E. Shannon, "Coding theorems for a discrete source with a fidelity criterion," IRE Nat. Conv. Rec, vol. 4, no. 142-163, p. 1, 1959.

[264] A. Segall, "Bit allocation and encoding for vector sources," IEEE Trans. Inf. Theory, vol. 22, no. 2, pp. 162-169, 1976. [Online]. Available: http://ieeexplore.ieee.org/stamp/stamp.jsp?arnumber $=1055533$

[265] T. Lookabaugh and R. Gray, "High-resolution quantization theory and the vector quantizer advantage," IEEE Trans. Inf. Theory, vol. 35, no. 5, pp. 1020-1033, 1989. [Online]. Available: http: //ieeexplore.ieee.org/stamp/stamp.jsp?arnumber $=42217$

[266] J. MacQueen et al., "Some methods for classification and analysis of multivariate observations," in Proceedings of the fifth Berkeley symposium on mathematical statistics and probability, vol. 1, no. 14. California, USA, 1967, pp. 281-297.

[267] Y. Linde, A. Buzo, and R. Gray, "An algorithm for vector quantizer design," IEEE Trans. Commun., vol. 28, no. 1, pp. 84-95, 1980. [Online]. Available: http://ieeexplore.ieee.org/stamp/stamp.jsp?arnumber $=1094577$

[268] M. J. Sabin and R. Gray, "Global convergence and empirical consistency of the generalized Lloyd algorithm," IEEE Trans. Inf. Theory, vol. 32, no. 2, pp. 148-155, 1986. [Online]. Available: http://ieeexplore.ieee.org/stamp/ stamp.jsp?arnumber $=1057168$

[269] V. K. Jones and G. C. Raleigh, "Channel estimation for wireless OFDM systems," in Proc. Bridge to Global Integration. IEEE Global Telecommunications Conf. GLOBECOM 1998, vol. 2, 1998, pp. 980-985. [Online]. Available: http://ieeexplore.ieee.org/stamp/stamp.jsp?arnumber $=$ 776875

[270] H. Feng and M. Effros, "On the rate-distortion performance and computational efficiency of the Karhunen-Loève transform for lossy data compression," IEEE Trans. Image Process., vol. 11, no. 2, pp. 113-122, 2002. [Online]. Available: http://ieeexplore.ieee.org/stamp/stamp.jsp?arnumber $=$ 982819

[271] 3GPP TR 25.814 V7.1.0, "Physical layer aspects for evolved Universal Terrestrial Radio Access (Release 7)." 
[272] S.-G. Hwang, "Cauchy's interlace theorem for eigenvalues of hermitian matrices," American Mathematical Monthly, vol. 111, no. 2, pp. 157-159, 2004. [Online]. Available: http://www.jstor.org/stable/10.2307/4145217

[273] Z. Bai, J. Demmel, J. Dongarra, A. Ruhe, and H. van der Vorst, Templates for the Solution of Algebraic Eigenvalue Problems: A Practical Guide. SIAM, Philadelphia, 2000.

[274] A. M. Vidal, V. M. Garcia, P. Alonso, and M. O. Bernabeu, "Parallel computation of the eigenvalues of symmetric toeplitz matrices through iterative methods," J. Parallel Distrib. Comput., vol. 68, no. 8, pp. 1113-1121, Aug. 2008. [Online]. Available: http://dx.doi.org/10.1016/j. jpdc.2008.03.003

[275] I. S. Dhillon, "A new $O\left(n^{2}\right)$ algorithm for the symmetric tridiagonal eigenvalue/eigenvector problem," Ph.D. dissertation, EECS Department, University of California, Berkeley, Oct. 1997. [Online]. Available: http://www.eecs.berkeley.edu/Pubs/TechRpts/1997/5888.html

[276] 3GPP TR 36.814 V9.0.0, "Evolved universal terrestrial radio access (EUTRA); Further advancements for E-UTRA physical layer aspects."

[277] G. J. Sullivan, "Efficient scalar quantization of exponential and laplacian random variables," IEEE Trans. Inf. Theory, vol. 42, no. 5, pp. 13651374, 1996. [Online]. Available: http://ieeexplore.ieee.org/stamp/stamp. jsp?arnumber $=532878$

[278] H. Minn and N. Al-Dhahir, "Optimal training signals for MIMO OFDM channel estimation," IEEE Trans. Wireless Commun., vol. 5, no. 5, pp. 1158-1168, 2006. [Online]. Available: http://ieeexplore.ieee.org/stamp/ stamp.jsp?arnumber $=1633369$

[279] T. A. Thomas, B. Mondal, and A. Ghosh, "CSI reference signal designs for enabling closed-loop MIMO feedback," in Proc. IEEE 72nd Vehicular Technology Conf. Fall (VTC 2010-Fall), 2010, pp. 1-5. [Online]. Available: http://ieeexplore.ieee.org/stamp/stamp.jsp?arnumber $=5594597$

[280] T. Koivisto, K. Schober, T. Kuosmanen, T. Roman, and M. Enescu, "Reference signal design for flexible MIMO operation in LTE-Advanced downlink," in Proc. IEEE 71st Vehicular Technology Conf. (VTC 2010Spring), 2010, pp. 1-5. [Online]. Available: http://ieeexplore.ieee.org/ stamp/stamp.jsp?arnumber $=5493965$

[281] X. G. Doukopoulos and R. Legouable, "Robust channel estimation via FFT interpolation for multicarrier systems," in Proc. VTC2007-Spring Vehicular Technology Conf. IEEE 65th, 2007, pp. 1861-1865. [Online]. Available: http://ieeexplore.ieee.org/stamp/stamp.jsp?arnumber $=4212814$ 
[282] A. Ancora, C. Bona, and D. T. M. Slock, "Down-sampled impulse response least-squares channel estimation for LTE OFDMA," in Proc. IEEE Int. Conf. Acoustics, Speech and Signal Processing ICASSP 2007, vol. 3, 2007. [Online]. Available: http://ieeexplore.ieee.org/stamp/stamp.jsp?arnumber $=4217704$

[283] C.-N. Chuah, D. Tse, J. Kahn, and R. Valenzuela, "Capacity scaling in MIMO wireless systems under correlated fading," IEEE Trans. Inf. Theory, vol. 48, no. 3, pp. 637-650, 2002. [Online]. Available: http://ieeexplore.ieee.org/stamp/stamp.jsp?arnumber $=985982$

[284] C. Polprasert and J. Ritcey, "A Nakagami fading phase difference distribution and its impact on BER performance," IEEE Trans. Wireless Commun., vol. 7, no. 7, pp. 2805-2813, 2008. [Online]. Available: http://ieeexplore.ieee.org/stamp/stamp.jsp?arnumber $=4570247$

[285] A. Forenza, D. J. Love, and R. W. Heath, "Simplified spatial correlation models for clustered MIMO channels with different array configurations," IEEE Trans. Veh. Technol., vol. 56, no. 4, pp. 1924-1934, 2007. [Online]. Available: http://ieeexplore.ieee.org/stamp/stamp.jsp?arnumber $=4277071$

[286] M. Abramowitz and I. A. Stegun, Handbook of Mathematical Functions, With Formulas, Graphs, and Mathematical Tables, ninth dover printing, tenth gpo printing ed. New York: Dover Publications, Incorporated, 1974.

[287] V. Aalo, G. Efthymoglou, and C. Chayawan, "On the envelope and phase distributions for correlated gaussian quadratures," IEEE Commun. Lett., vol. 11, no. 12, pp. 985-987, 2007. [Online]. Available: http://ieeexplore.ieee.org/stamp/stamp.jsp?arnumber $=4400760$

[288] W. Pearlman, "Polar quantization of a complex Gaussian random variable," IEEE Trans. Commun., vol. 27, no. 6, pp. 892-899, 1979. [Online]. Available: http://ieeexplore.ieee.org/stamp/stamp.jsp?arnumber $=1094476$

[289] J. Lagarias, J. Reeds, M. Wright, and P. Wright, "Convergence properties of the Nelder-Mead simplex method in low dimensions," SIAM Journal on Optimization, vol. 9, no. 1, pp. $112-147,1998$. [Online]. Available: http://epubs.siam.org/doi/abs/10.1137/S1052623496303470

[290] P. Castro, M. Joham, L. Castedo, and W. Utschick, "Robust precoding for multi-user MISO systems with limited-feedback channels," in Proceedings of the International ITG Workshop on Smart Antennas, Vienna, Austria, 2007.

[291] R. A. Horn and C. R. Johnson, Matrix Analysis. Cambridge University Press, 2012.

[292] 3GPP TR 36.213 V11.4.0, "Physical layer procedures (Release 11)." 
[293] T. J. Willink, "Limits on estimating autocorrelation matrices from mobile mimo measurements," International Journal of Antennas and Propagation, vol. 2013, p. 6 pages, 2013. [Online]. Available: http: //www.hindawi.com/journals/ijap/2013/345908/abs/

[294] S. Ghosh, B. Rao, and J. Zeidler, "Techniques for MIMO channel covariance matrix quantization," IEEE Trans. Signal Process., vol. 60, no. 6, pp. 3340-3345, 2012. [Online]. Available: http://ieeexplore.ieee.org/stamp/ stamp.jsp?arnumber $=6168854$ 